\title{
Helicobacter small RNA regulates host adaptation and carcinogenesis
}

Ryo Kinoshita-Daitoku¹,2, Kotaro Kiga², Ryota Otsubo ${ }^{1}$, Yoshitoshi Ogura ${ }^{3}$, Takahito Sanada ${ }^{1,2}$, Zhu Bo ${ }^{2}$, Tuan Vo Phuoc ${ }^{4,5}$, Tokuju Okano ${ }^{6}$, Tamako lida², Rui Yokomori ${ }^{7}$, Eisuke Kuroda ${ }^{1,2}$, Sayaka Hirukawa ${ }^{2}$, Mototsugu Tanaka $^{2,8}$, Arpana Sood ${ }^{2}$, Phawinee Subsomwong ${ }^{1}$, Hiroshi Ashida ${ }^{6}$, Tran Thanh Binh ${ }^{4,5}$, Lam Tung Nguyen ${ }^{4}$, Khien Vu Van ${ }^{9}$, Dang Quy Dung $\mathrm{Ho}^{5}$, Kenta Nakai ${ }^{7}$, Toshihiko Suzuki ${ }^{6}$, Yoshio Yamaoka ${ }^{4}$, Tetsuya Hayashi ${ }^{3}$, and Hitomi Mimuro ${ }^{1,2}$

1 Department of Infection Microbiology, Research Institute for Microbial

Diseases, Osaka University, Osaka, Japan.

2 Division of Bacteriology, Department of Infectious Diseases Control, International Research Center for Infectious Diseases, The Institute of Medical Science, The University of Tokyo, Tokyo, Japan.

3 Department of Bacteriology, Graduate School of Medical Sciences, Kyushu University, Fukuoka, Japan.

4 Department of Environmental and Preventive Medicine, Faculty of Medicine, Oita University, Yufu, Oita, Japan.

5 Department of Endoscopy, Cho Ray Hospital, Ho Chi Minh 749000, Vietnam.

6 Department of Bacterial Pathogenesis, Infection and Host Response, Graduate School of Medical and Dental Sciences, Tokyo Medical and Dental University, Tokyo, Japan.

7 Human Genome Center, The Institute of Medical Science, The University of Tokyo, Tokyo, Japan.

8 Division of Nephrology and Endocrinology, The University of Tokyo School of Medicine, Tokyo, Japan 
9 Department of GI Endoscopy, 108 Central Hospital, Hanoi, Vietnam. 


\begin{abstract}
Type-1 carcinogenic Helicobacter pylori that is known to evolve during longterm infection, enters the stomach orally and causes gastric cancer using the carcinogenic protein $\mathrm{CagA}^{1}$. However, little is known about the adaptation mechanisms of $H$. pylori when the environment changes from the outside to the inside of the living body. Here we show that small non-coding RNA HPnc4160 is a crucial novel RNA molecule of $H$. pylori that negatively regulates bacterial-host adaptation and gastric cancer. $H$. pylori isolated from gerbil's stomachs eight weeks post-infection acquired mutations in the increased number of T-repeats within the upstream region of the HPnc4160 coding region, which leads to reduced HPnc4160 expression levels that also seen in cancer patients-derived $H$. pylori. By comparing RNA-seq and iTRAQ analysis between wild-type and hpnc4160 deficient mutant strains, we identified eight targets of HPnc4160 including cagA and unknown factors. Mice infection experiment revealed that the hpnc4160 deficient mutant had a higher number of colonized bacteria in the mice stomach than the wild-type strain, indicating that reduced expression levels of HPnc4160 was important for bacterial host adaptation. The expression level of HPnc4160 was lower in the clinical isolates derived from gastric cancer patients compared with noncancer-derived strains, while the mRNA expression levels of target factors were higher. Our findings highlight the first discovery that HPnc4160 is an important small RNA for bacteria to adapt to the host environment leading to gastric carcinogenesis.
\end{abstract}




\section{Main}

Helicobacter pylori infection has a very high prevalence that about half of the world population is infected. Patients infected with CagA-positive H. pylori closely related to disease malignancy have been reported to have an increased risk of peptic ulcer, chronic gastritis, intestinal metaplasia, and gastric cancer. ${ }^{2,3}$ The highly pathogenic $H$. pylori possess a cag pathogenicity island that encodes components of a Type IV secretion system (TFSS), which is an injection needle, and a carcinogenic factor CagA effector protein. The $H$. pylori that has reached the gastric epithelium injects CagA protein, peptidoglycan, and heptose-1,7-bisphosphate into the attached cells via TFSS, and stimulates signal transduction pathways such as NF-KB to promote production of chemokines such as interleukin-8 (IL-8) ${ }^{4-11}$.

It is considered that the genetic diversity at the genomic level that is characteristic of $H$. pylori is very important to establish a persistent infection in an infected host with different backgrounds, adapting to a gastric niche with severe environmental changes ${ }^{12}$. The $H$. pylori gene mutations are characterized by the presence of simple repetitive sequences such as mononucleotide repeats (such as poly-T) and dinucleotide repeats (such as CT-repeats). From the analysis of clinical isolates of $H$. pylori so far, it is considered that phase variations are induced by ON / OFF control of gene expression of such as outer membrane proteins (OMPs) due to expansion and contraction of these simple repetitive sequences ${ }^{12-14}$. In the course of chronic infection, in order to escape from the host immunity, it is assumed that strong diversity in $H$. pylori OMPs that can serve as highly antigenic cell surface antigens will cause a strong directivity of selection ${ }^{15}$. Therefore, to understand the mechanism of persistent infection by $H$. pylori, we analyzed bacterial gene mutations acquired by $H$. pylori in the course of persistent infection using an 
experimental animal infection system with the same host genetic background. With growing evidence that bacterial small RNA (sRNA)-mediated target gene expression in response to changes in the environment, we focused sRNAs as well ${ }^{16}$.

\section{Identification of HPnc4160}

To analyze the bacterial gene mutation acquired by $H$. pylori during the persistent infection, Mongolian gerbils $(n=10)$ were inoculated with $H$. pylori ATCC 43504 wild-type strain for 8 wks. H. pylori in the infected stomachs were isolated ( $n=40 ;$ Fig. 1a), and analyzed comparative whole genome sequences (Fig. 1b, Supplementary Information 1, 2). We totaled genomic positions, where these mutations were introduced, for each coding and intergenic region, and identified 13 regions (Regions R1, R3-R5, R7-R8, R10-R16) where mutations were introduced in $50 \%$ or more of the strains (Fig. 1b, Extended Data Table 1).

To investigate whether the mutated region affects RNA expression in isolates recovered from the gerbils, the expression levels of mRNA or noncoding RNA around the mutated regions were quantified by quantitative PCR. Among the corresponding 15 CDSs and non-coding RNA (HP0947, babA, tpiA, jhp1163, HP0811, HPnc4160, HPnc4170, jhp0540, araS, pldA, sabA, HP1354, hopZ, tIpB, HPB8_818), we found that the expression level of HPnc4160, a non-coding small RNA (sRNA) of unknown function, fluctuated the most compared to the wild-type (Fig. 1c, Extended Data Fig. 1a). Similar results were also obtained with the strains $(n=10)$ isolated from C57BL/6 mice $(n=5)$ (Fig. 1b, Extended Data Fig. 1a, b; Supplementary Information 3, 4).

Region R14 is the upstream region of HPnc4160 and HP0811, and is located in the CDS of HPnc4170 (aapB small ORF homologue) encoded by the complementary sequence of HPnc4160 (Fig. 1d) ${ }^{17}$. HPnc4160 and its 
upstream T repeat were conserved in various $H$. pylori genome analysis strains, and T repeat length was different depending on the strain (Extended Data Fig. 2a). A repeat of 2 to 4 bases of thymidine was inserted into the repeat of isolates from rodents, and the repeat length increased depending on the infection period (Fig. 1e, Extended Data Fig. 2b-e). Importantly, sequence analysis of clinical isolates showed that $\mathrm{T}$ repeat lengths were longer in cancer patient-derived strains than in non-cancer patient-derived strains (Fig. 1f, Supplementary Information 5). However, expansion of the repeat was not observed in long-term in vitro culture (Extended Data Fig. 3). Next, we analyzed the change in HPnc4160 sRNA expression levels by T repeat length. In strains recovered from $H$. pylori-infected rodent stomachs, HPnc4160 expression level tended to decrease with expansion of T repeat length (Fig. 1g). To exclude the effects of mutations other than the T repeats, we further analyzed RNA expression levels of HP0811, HPnc4170 and HPnc4160 in various mutants in which the T repeat sequence was inserted into the HPnc4160 upstream region of wild-type strain (T15mut, T16mut, T17mut, T18mut, and T19mut). In strains in which the number of T repeats was greater than T14 of wild-type, the expression levels of HPnc4160, but not HP0811 and HPnc4170, were significantly reduced compared with wild-type (Fig. 1h, Extended Data Fig. 4a-c,). Although HPnc4160 and HPnc4170 were initially reported as the small ORF-encoding mRNA/antisense RNA family aapB/IsoB, in which the Iso transcript acts as asRNA antitoxin to modify the aap expression $^{18}$, our data indicated that HPnc4160 expression levels had no effect on HPnc4170 levels. These results indicated that expression levels of HPnc4160 sRNA decreased when the number of Region R14 T repeats increased due to persistent intragastric infection.

\section{Identification of HPnc4160 target genes}


Many sRNAs regulate the expression of a target mRNA by specifically binding to a complementary sequence in the target protein coding mRNA. To elucidate the target mRNA of HPnc4160, we made a $\Delta$ hpnc4160/hpnc4170 strain, in which both the HPnc4160 and the HPnc4170 on the complementary strand were deleted, and analyzed comparative mRNA and protein expression. We identified eight factors (cagA, hofC, HELPY_1262, hpaA, horB, omp14, hopE, and HP1227) with P-values lower than 0.001 (RNA-seq analysis) and 0.01 [isobaric tag for relative and absolute quantitation (iTRAQ) labeling and LCMS/MS analysis] (Fig. 2a-c, Extended Data Table 2a-b). Of these, cagA was prominent in expression levels of mRNA and protein.

We analyzed whether the mRNA expression levels of the identified eight factors depend on the presence of HPnc4160. Although the expression level of HPnc4160 showed a decreasing trend with T16mut as the lowest value, the mRNA of the eight candidates showed increasing trends with T16mut as the highest value. The Spearman correlation coefficients $r$ between the expression levels of these target mRNAs and HPnc4160 showed a very strong inverse correlation from -0.7714 to -1.0 (Extended Data Fig. 4d).

Next, we examined the mRNA expression correlation between HPnc4160 and each factor. The HPnc4160 overexpression strain (WT / pHel2hpnc4160) significantly increased the expression level of HPnc4160 compared to the wild-type strain, but significantly decreased the expression level of each factor mRNA. On the other hand, in $\Delta$ hpnc4160/hpnc4170 strain, mRNA expression of each target increased compared to the wild-type. Since Shpnc4160/hpnc4170 strain also lacks the HPnc4170 sequence present in the complementary strand of HPnc4160, we constructed a Ahpnc4160/hpnc4170 /pHel2-hpnc4160 strain complementing only HPnc4160 to confirm the effect of the HPnc4170 sequence on HPnc4160 target mRNA expression. Compared to the $\Delta$ hpnc4160/hpnc4170 strain, the mRNA expression levels of the 
candidates were decreased in the HPnc4160 complemented shpnc4160/hpnc4170 /pHel2-hpnc4160 (Fig. 2d, Extended Data Fig. 5a). These data indicated that the expression levels of the HPnc4160 target 8 candidates were suppressed depending on the expression level of HPnc4160.

When sRNA binds within a few bases around the 5 'UTR or start codon of target RNA, it often competes with ribosomes and inhibits translation initiation. If the sRNA binds within the CDS far downstream of the initiation codon, it causes mRNA degradation by RNase E or RNase III to suppress translational activation ${ }^{19}$. We confirmed by electrophoretic mobility shift assays (EMSA) that HPnc4160 binds to the 5 'UTR of seven genes other than cagA (Fig. 3a). In seven factors other than $\operatorname{cag} A$, we confirmed a sequence complementary to the HPnc4160 sequence in the 5 'UTR of each gene (Extended Data Fig. 5b-c). In the cagA gene, we found HPnc4160-binding sequences Type 1 at one position (2344 nt), and Type 2 at four positions (2838, 2940, 3042, and 3144 nt) within the CM/CRPIA motifs in CagA Cterminal region, which is known to bind with host signal proteins ${ }^{7,20}$ (Fig. 3b, Extended Data Fig. 5d-f). We confirmed direct binding of cagA partial CDS (positions 2778 to $3236 \mathrm{nt}$ from start codon of cagA) to HPnc4160 (Fig. 3c). The binding between the two was abolished in the NB-cagA RNA in which the HPnc4160 binding sequence was mutated at four positions (Type 2) while the amino acid sequence of CagA was preserved (Fig. 3c, Extended Data Fig. 5g). In addition, we found that in the presence of $H$. pylori RNase III recombinant protein, the binding between HPnc4160 and biotin-labeled partial cagA mRNA, but not NB-cagA RNA, disappeared (Fig. 3d, Extended Data Fig. 5i). These data clearly demonstrated that HPnc4160 controls cagA at the posttranscription level by binding to multiple binding sequences present in its CDS region, and promotes degradation by RNase III. 


\section{Effects of HPnc4160 on H. pylori pathogenicity}

Among the factors that HPnc4160 regulated the expression levels, we further analyzed CagA, which has been deeply involved in pathogenesis. First, we confirmed whether the binding of HPnc4160-cagA mRNA actually controls the expression levels of $\operatorname{cag} A$ mRNA and protein in $H$. pylori. The quantitative PCR showed that, in the H. pylori expressing NB-cagA in which all five HPnc4160binding DNA sequences were mutated but the amino acid sequence was preserved (Extended Data Fig. 5g-h), the expression level of HPnc4160 was similar to that of the wild-type, but the expression level of $\operatorname{cag} A$ mRNA was significantly increased to the same extent as that of the $\Delta h p n c 4160 / h p n c 4170$ strain (Extended Data Fig. 6a, Fig. 4a). Using the urease UreA protein as a loading control for $H$. pylori, we confirmed that NB-cagA strain expressed CagA protein at a higher level than wild-type and $\Delta$ hpnc4160/hpnc4170 / pHel2-hpnc4160 strains, similar to $\Delta$ hpnc4160/hpnc4170 strain (Fig. 4b). Next, we analyzed Western blot of the gastric epithelial cell line AGS infected with $H$. pylori. Using $\beta$-actin as a loading control for cells, we confirmed that the amount of UreA protein, which exhibited the bacterial amounts, was the same in the lysates of cells infected with any of the strains, indicating that all of the strains showed same binding ability to AGS cells (Extended Data Fig. 6b). Some of the CagA proteins injected from $H$. pylori to host epithelium via TFSS were tyrosine phosphorylated by host Src/Abl kinase and detected by pYCagA-specific antibody. Using the antibody, we confirmed that the amount of intracellular CagA was increased in the NB-cagA-infected cells, accompanied with the increase in the amount of CagA (Extended Data Fig. 6b). Injected CagA induces AGS cell motility (scattering/hummingbird). In the AGS cells infected with the $\Delta$ hpnc4160/hpnc4170 or the NB-cagA strains, more remarkably elongated cells were observed than in the wild-type or shpnc4160/hpnc4170 / pHel2-hpnc4160 strain-infected cells (Fig. 4c-d). When 
we analyzed amount of IL-8 protein secreted from $H$. pylori-infected cells, which is induced mainly by intracellular CagA, we found that the cagA-NB strain infected cells had higher IL-8 producing ability than the wild-type infected cells (Extended Data Fig. 6c). These results suggested that binding of HPnc4160 to cagA mRNA is important for controlling the amount of functional CagA protein injected by $H$. pylori.

To understand the significance of the HPnc4160 control mechanism in the bacterial adaptation to the host to establish infection, mice were orally inoculated with each strain, and the number of bacteria colonized in the stomach was analyzed three days post infection. The number of colonized bacteria in the stomach was significantly increased in the Lhpnc4160/hpnc4170 strain compared to the wild-type, but the Shpnc4160/hpnc4170 / pHel2-hpnc4160 and the NB-cagA strains were equivalent to wild-type (Fig. 4e). Since $\Delta$ hpnc4160/hpnc4170 infection significantly increased Cxc/2 mRNA compared to wild-type infection, but NB$\operatorname{cag} A$ strain was equivalent to wild-type, it is suggested that factors other than CagA controlled by HPnc4160 may be important for the bacterial adaptation as well as development of gastritis (Fig. 4f). To confirm the significance of HPnc4160 in the pathogenesis of $H$. pylori, we examined the expression levels of the HPnc4160 target genes in clinical isolates. As shown in Fig. 4g, isolates from cancer patients had lower levels of hpnc4160, but increased expression of six factors controlled by HPnc4160 (cagA, horB, hopE, omp14, hofC, and hpaA), compared to isolates from non-stomach cancer patients (Fig. $4 \mathrm{~g}$, Extended data Fig. 7). These data strongly suggested that target mRNA expression was suppressed by HPnc4160 at the onset of $H$. pylori infection, and with the course of infection, thymidine repeats were inserted into the upstream region of HPnc4160, HPnc4160 expression decreased, and target mRNA expression increased; those episodes contributed to bacterial 
adaptation to the host environment, leading to gastritis and gastric cancer formation (Extended Data Fig. 8).

\section{Discussion}

Among the bacterial factors, CagA had extremely high mRNA and protein levels in the bacterial cells (Fig. $2 a$ and b). Our data indicated that T-stretch length in the upstream region of hpnc4160 did not elongate under in vitro condition (Extended Data Fig. 3). However, the T-stretch length was increased and the expression levels of CagA and OMPs increased in vivo in gastric infection (Fig. 1e, 2a and 2b). Adaptation to the in vitro culture environment does not require CagA or OMPs. Therefore, under in vitro conditions, it may be that the expression level of hpnc4160 is increased and the expression of target is suppressed in order to allocate energy for the growth of bacterial cells, rather than expressing genes having high expression levels such as CagA. CagA suppresses apoptosis of the gastric mucosal epithelium and contributes to persistent infection of $H$. pylorib. When $H$. pylori enter the stomach, the bacteria may have acquired a mechanism to decrease the expression of HPnc4160 to increase the expression of OMP and CagA simultaneously, in order to adapt to environmental changes to colonize the gastric mucosa for a scaffold for growth.

Gene expression control mechanism by variation of the number of repeat sequences is known as one of various gene expression control mechanisms by phase variation in $H$. pylori ${ }^{21}$. This study suggests that the repeat sequence of $H$. pylori genome is important not only as an ON / OFF mechanism of protein expression such as cell adhesion factors SabA and BabA, but also in sRNA expression. It has been reported that $H$. pylori DNA polymerase I does not have gene repairing activities like other bacteria, thus 
insertions or deletions of bases called slipped strand mispairing (SSM) occur in simple repetitive sequences ${ }^{22}$. In fact, since there were variations in poly-T length within the upward region of hpnc4160 in $\mathrm{H}$. pylori in vivo (Extended Data Fig. 2a), it is conceivable that $H$. pylori become genetically heterogeneous bacterial population using SSM during the course of infection, so that a population suitable for mutation in the host is selected and propagated. In this study we mainly used strain ATCC 43504, which is a clinical isolate originally from the human antrum. The poly-T length, which did not fluctuate in the in vitro subculture, increased in rodent isolates ranged from 14 to 19 copies (Extended Data Fig. 2, 3), indicating that the introduction of SSM may be caused by some host stress condition.

The HPnc4160-binding sequence in the ATCC 43504 cagA CDS was duplicated at five sites (Fig. 3b). In the regulation of mRNA expression by general sRNA, mRNA instability can be induced by binding at one site. This is the first report of a sRNA gene regulatory mechanism having multiple binding sequences in one gene of a pathogen. The four out of five HPnc4160-binding sequences in the cagA CDS was located in the CM/CRPIA motif of the cagA gene, which is involved in maintaining host epithelial cell structure ${ }^{20}$. Since the number of the CM/CRPIA motif differs depending on the strain, HPnc4160 may regulate differences in pathogenicity between Western and East Asian type of H. pylori.

The onset of the diseases due to $H$. pylori infection is thought to be the result of persistent infection for decades after the initial infection during childhood, when a very small number of bacteria-containing aerosol were taken into the body orally ${ }^{23}$. However, since it is difficult to analyze $H$. pylori infection experiments using laboratory animals for decades, a relatively short time infection analysis must be performed by inoculating a large number of bacteria. Therefore, the mutation analysis in this study may correspond to 
mutations acquired in the acute phase (Supplementary information 1 and $3)^{15},{ }^{24}$. Infection by a small number of bacteria that mimic natural infection allows for more detailed analysis of the establishment of infection.

In this study, we investigated the mutations of $H$. pylori, originated from the same strain, infected in experimental animals with the same genetic background and environment. Unlike studies in individuals with widely differing genetic backgrounds, stomach environments, and infection history, our study has advantage for understanding the adaptation process of $H$. pylori to the host. We discovered a novel non-coding sRNA that is important for posttranscriptional translation control of pathogenic factors of $H$. pylori, such as CagA, which was previously considered to be the most important pathogenic factor for gastric cancer development, and putative OMPs involved in bacterial adhesion. This study is not limited to elucidating the complicated mechanism of persistent $H$. pylori infection, but its application to $H$. pylori-specific therapies that do not rely on antibiotics can also be expected. 


\section{Figure legends}

Fig. 1: H. pylori acquire poly-T extension in upstream of HPnc4160 small RNA to decrease its expression during infection in vivo.

a, Experimental strategies schematic. b, Circular genomic map of ATCC 43504 strain recovered from stomachs of gerbils and mice. c, Mutation rates and expression levels of candidate RNAs (mRNA or non-coding RNA). RNA expression levels of the ORFs or nearby genes of genome regions [total 15 genes (Extended Data Fig. 1a), which mutated in more than $50 \%$ of the recovered strains from the gerbils, were assessed and plotted against the mutation rates. d, Schematic structures of genes around HPnc4160. e, Schematic diagram of genome DNA sequence around the HPnc4160 and polyT sequence of recovered strains harboring mutations. Green indicates HPnc4160 transcribed sequence, red frame indicates poly-T mutated stretches in the upstream region of HPnc4160 of ATCC 43504 wild-type (WT) strain, and red-colored "T" indicated inserted nucleotides of each recovered strain compared with WT. f, The T-repeat length in the upstream region of HPnc4160 of clinical isolates. Isolated strains from cancer patients (Cancer) have a higher number of T-repeat in the upstream region of Hpnc4160 compared with the isolates from non-cancer patients (Non-Cancer). Data are presented as means with $95 \%$ confidence interval. $P$ values represent the results of the two-tailed Mann-Whitney test. g, Expression levels of HPnc4160 in the recovered $H$. pylori strains from Mongolian gerbils $(n=40)$. Relative expression levels of HPnc4160 were measured by real-time PCR and plotted against T-repeat length in the upstream region of HPnc4160. Data are presented as means with s.d. $P$ values represent the results of two-tailed Dunn's multiple comparison test. $\mathbf{h}$, The relative expression levels of HPnc4160 in the $H$. pylori strains genetically modified with the T-repeat length. Data are presented as means 
with s.d. (n=3). P values are from Dunnett's multiple comparison test (at twosided).

Fig. 2: HPnc4160 downregulates expression levels of bacterial pathogenic factors.

a, The MA plot of ratios [ $\Delta$ hpnc4160/hpnc4170 / wild-type (WT) H. pylori] versus their normalized average mRNA expression determined by RNAsequencing (RNA-Seq). The red dots showed genes of $P<0.001$. b, Volcano plots of the proteins quantified by isobaric tags for relative and absolute quantification (iTRAQ) analysis comparing WT and $\Delta h p n c 4160 / 4170$. Each point represents the difference in expression (fold change) between the two groups plotted against the level of statistical significance. The red dots showed proteins of $P<0.01$. c, The Venn diagram represents the number of factors whose expression exhibited significant differences between Shpnc4160/hpnc4170 and WT. d, The relative RNA expression levels of target candidates of HPnc4160 showed an inverse correlation with HPnc4160. The results represent the average of three separate experiments (each $n=3$ ). Data are presented as means \pm s.d. (error bars). $P$ values are from Tukey's multiple comparison test (at two-sided). ns: not significant.

Fig. 3: HPnc4160 binds to target mRNA.

a, Electrophoretic mobility shift assay (EMSA) analysis of HPnc4160 binding to the 5' UTR region of each candidate mRNA. b, Schematic of CagA motifs, and HPnc4160 binding sequences. c, EMSA analysis of HPnc4160 binding to RNA of partial cagA WT or HPnc4160-non-binding $\operatorname{cag} A$ (NB-cagA). d, RNase protection assay with HPnc4160, cagA mRNA, and recombinant RNase III.

Fig. 4: HPnc4160 controls bacterial host adaptation and pathogenesis. 
a, HPnc4160, and cagA mRNA expression levels. The results represent the average of three separate experiments (each $n=3$ ). Data are presented as means \pm s.d. (error bars). $P$ values are from non-parametric Dunnett's multiple comparison test (at two-sided). ns: not significant. Experiments were repeated three times with similar results. $\mathbf{b}$, Protein expression levels of CagA in each mutant strain. UreA protein levels serve as bacterial loading controls. c and d, Scattering phenotypes of $H$. pylori-infected AGS gastric epithelial cells. c, DNA (blue), F-actin (red), and anti-phosphorylated CagA antibody (pY-CagA, green) were stained. Scale bar, $50 \mu \mathrm{m}$. d, Quantification of scattering activity of AGS cells induced by $H$. pylori infection. e and f, HPnc4160 deletion mutants efficiently colonized the stomach and contributed to increasing mRNA levels of inflammatory chemokine $\mathrm{Cxc} / 2$ in mice stomach. C57BL/6 mice were inoculated with $H$. pylori. At three days after infection, animals were sacrificed, and a quantitative culture assay (e) and a quantitative RT-PCR (f) were performed on gastric specimens. Data are median with interquartile range. $P$ values are from non-parametric Dunn's multiple comparison test (at two-sided). ns: not significant. g, Clinical isolates from malignant patients downregulate HPnc4160 and upregulate expression levels of its target genes. Expression levels of indicated mRNAs in clinical isolates of non-cancer (Non-Cancer, $\mathrm{n}=39$ ) and cancer (Cancer, $\mathrm{n}=17$ ) patients were quantified and normalized with the levels of $23 \mathrm{~S}$ rRNA. Data are presented as medians with interquartile range. $P$ values are from the non-parametric Mann-Whitney test (at two-sided). 


\section{Methods}

\section{Data reporting}

No statistical methods were used to predetermine sample size, and the experiments were not randomized and the investigators were not blinded to allocation during experiments and outcome assessment.

\section{Strains and culture conditions}

The Helicobacter pylori strain ATCC 43504, its isogenic mutants $\triangle c a g A$ and $\Delta$ virB7, strains SS1 and PMSS1 have been described previously ${ }^{6,25}$. H. pylori was cultured on Trypticase soy agar with 5\% (v/v) sheep blood (Thermo Fisher Scientific, Waltham, MA, USA) for 2 days at $37^{\circ} \mathrm{C}$ in microaerobic conditions. Bacterial colonies were suspended in Brucella broth (Thermo Fisher Scientific) supplemented with 5\% (v/v) inactivated FBS (Thermo Fisher Scientific), adjusted to Optical density $600 \mathrm{~nm}$ of 0.05 , and incubated 15 hours at $37^{\circ} \mathrm{C}$ with gentle agitation under microaerobic conditions.

The AGS human gastric epithelial cell line (ATCC CRL-1739) was maintained in DMEM/F-12 (Thermo Fisher Scientific) containing 10\% (v/v) FBS. AGS cells were seeded in six-well plates and grown to $\sim 80 \%$ confluence to be used for western blot analysis. For immunofluorescence microscopy, cells were seeded in six-well plates with cover glass, and grown to $\sim 80 \%$ confluence.

\section{Antibodies and immunohistochemical reagents}

The anti-Tyr(P)-CagA, and anti-UreA polyclonal antibodies have been described previously (Mimuro MC 2002). Anti-CagA polyclonal antibody was purchased from AUSTRAL Biologicals (CA, USA), anti-actin monoclonal antibody was from MERCK (Darmstadt, Germany), Horse radish peroxidase (HRP)-labeled anti-rabbit IgG and HRP-labeled anti-mouse IgG, and FITC- 
labeled anti-rabbit IgG was from Jackson ImmunoResearch Laboratories Inc. (PA, USA). DAPI was from SIGMA-ALDRICH (MD, USA), and Rhodamine Phalloidin was from Thermo Fisher SCIENTIFIC (MA, USA).

\section{Animal infection}

H. pylori infection of rodents were performed as described previously ${ }^{26}$. Briefly, 6-week-old male MON/Jms/GbsSlc Mongolian gerbils were orally administered with $200 \mu \mathrm{L}$ of Vancomycin $(500 \mathrm{mg} / \mathrm{L})$ at 24 and 48 hours before $H$. pylori inoculation. On the days of $H$. pylori inoculation, $300 \mu \mathrm{L}$ of $5 \%(w / v)$ sodium bicarbonate were orally administrated 10 minutes before bacterial inoculation. The gerbils were then intragastrically inoculated with an $H$. pylori culture containing $10^{9} \mathrm{CFU}$ for 2 consecutive days. As for C57BL/6 mice (SLC Japan Inc., Tokyo, Japan) were intragastrically inoculated once with $H$. pylori culture of $10^{9} \mathrm{CFU}$. After indicated date, the stomach of each infected animal was opened along the greater curvature. To quantitatively isolate $H$. pylori, the stomach was excised, weighed, and homogenized. Serial dilutions were plated on H. pylori-selective agar plates (Eiken Chemical Co.) and incubated under microaerophilic conditions at $37^{\circ} \mathrm{C}$ for 4 days, after which the cfu were counted. Colonization data points of $1 \times 10^{3}$ cfu were the minimal detection limit of the assay.

For isolation of strains recovered from $H$. pylori-infected rodents, each colony on the $H$. pylori-selective agar plates were picked up and spread on Trypticase soy agar with $5 \%(\mathrm{v} / \mathrm{v})$ sheep blood, and incubated under microaerophilic conditions at $37^{\circ} \mathrm{C}$ for two days. Then, the colonies were suspended in Brucella broth supplemented with $5 \%(\mathrm{v} / \mathrm{v})$ inactivated FBS, adjusted to Optical density $600 \mathrm{~nm}$ of 0.05 , and incubated 15 hours at $37^{\circ} \mathrm{C}$ with gentle agitation under microaerobic conditions. The cultures were preserved with $50 \%(\mathrm{v} / \mathrm{v})$ glycerol in $-80^{\circ} \mathrm{C}$ until use. 
For RNA isolation, the tissue was immediately frozen in liquid nitrogen. Animal experiments were conducted in accordance with the University of Tokyo or Osaka University guidelines for the care and use of laboratory animals and were approved by the ethics committee for animal experiments at the University of Tokyo or Osaka University.

\section{Genomic DNA purification and sequencing}

For PCR templates, genomic DNA was purified using InstaGene Matrix (BioRad Laboratories, Inc., CA, USA).

For whole genome sequencing, genomic DNA was purified from mid-log phase culture of strain ATCC43504 using QIAGEN DNeasy (QIAGEN). A genomic DNA library for sequencing was prepared using the Nextera XT DNA Sample Preparation kit (Illumina, San Diego, CA, USA) and sequenced using the Illumina MiSeq (for isolates from gerbils) or HiSeq $X$ (for isolates from mice) platform to generate 300-bp paired-end reads. Genome assembly, scaffolding, and gap-closing were performed using the Platanus assembler (Kajitani et al. 2014). Gene identification and annotation were conducted by the Microbial Genome Annotation Pipeline (MiGAP [http://www.migap.org]). The raw read sequences and assembled scaffold sequences have been submitted to the DDBJ/EMBL/Genbank under the Bioproject accession number; SAMD00178897- SAMD00178935, SAMD00179460, SAMD00178937 and SAMD00204457- SAMD00204466.

The DNA sequences mutated in more than $50 \%$ of the 40 strains recovered from Mongolian gerbils, or, in all of the 10 strains recovered from C57BL/6 mice were listed in Extended Data Table 1. We selected the genes to further analyze for their mRNA expression levels as follows. For the gene in which the mutation was in the CDS region, the mRNA expression level of the CDS was measured. While, when the mutation insertion region was an intergenic region, 
we measured the mRNA expression level of an adjacent gene in which the intergenic region could be a 5'UTR region. As for HP1243 and HPG27_298, which started from 3' end of HP1243 with 33 nucleotides spaces, were regarded as a continuous gene; since both genes are annotated as babA gene and ribosomal binding site (RBS) is assigned only at the upstream region of HP1243.

\section{In vitro passage experiment}

H. pylori ATCC 43504 was recovered from frozen stock and cultured on $5 \%$ $(\mathrm{v} / \mathrm{v})$ sheep blood agar for 2 days at $37^{\circ} \mathrm{C}$ in microaerobic conditions. Bacterial colonies were suspended in 3 tubes of Brucella broth supplemented with $5 \%$ $(v / v)$ inactivated FBS. Each bacterial suspension was adjusted to Optical density $600 \mathrm{~nm}$ of 0.05 , and incubated 12 hours at $37^{\circ} \mathrm{C}$ with gentle agitation under microaerobic conditions. Following this incubation, each fraction of the suspension was preserved by freezing in 50\% (v/v) glycerol as "Original" strains. Meanwhile, each bacterial suspension was sub-cultured by resuspending in Brucella broth supplemented with $5 \%(\mathrm{v} / \mathrm{v})$ inactivated FBS to adjust Optical density $600 \mathrm{~nm}$ of 0.05 , and incubated additional 12 hours at $37^{\circ} \mathrm{C}$ with gentle agitation under microaerobic conditions. The sub-cultivation was repeated for 60 passages (30 days), and each cell suspension was preserved by freezing in $50 \%$ (v/v) glycerol as "60-passaged" strains. The "Original" and "60-passaged" strains were recovered from frozen stock on 5\% ( $v / v)$ sheep blood agar by 2 days incubation under microaerobic conditions, and then the colonies were suspended in Brucella broth supplemented with 5\% $(\mathrm{v} / \mathrm{v})$ inactivated $\mathrm{FBS}$ and incubated 12 hours at $37^{\circ} \mathrm{C}$ with gentle agitation under microaerobic conditions. The bacterial cells were collected and subjected to the genomic DNA purification. 


\section{RT-PCR}

For preparation of total RNA from $H$. pylori, the liquid cultures of $H$. pylori were agitated under microaerobic conditions at $37^{\circ} \mathrm{C}$ overnight until the OD value at $600 \mathrm{~nm}$ reached 0.9 .

Total RNA was extracted using ISOGEN (Nippon Gene, Tokyo, Japan), according to the manufacturer's instructions. The concentration of the purified total RNA was analyzed using the NanoDrop Spectrophotometer (ThermoFisher Scientific, Wilmington, DE, USA). The total RNA was reverse transcribed into cDNA with miScript II RT Kit (QIAGEN) according to the manufacturer's instructions. The levels of mRNA expression were quantified and normalized to 23SrRNA (for H. pylori) or Gapdh (for mice) expression with a THUNDERBIRD SYBR qPCR (TOYOBO) using the primer pairs described in Supplementary Information 6 . The results are expressed as the means \pm SEM from triplicate strain experiments.

\section{Genetic manipulation}

Construction of plasmids for producing gene-deficient mutants Isogenic gene null mutants derived from ATCC 43504 were constructed by insertional mutagenesis as follows. Using the extracted H. pylori ATCC 43504 genome as a template, DNA fragments containing the upstream region $500 \mathrm{bp}$ and the downstream region 500 bp of the target gene were amplified by PCR using primer (CagA KO up Xhol, CagA KO up EcoRI, CagA KO down BamHICagA KO down Notl, HPnc4160/4170 KO up Kpnl, HPnc4160/4170 KO up Clal, HPnc4160/4170 KO down BamHI, HPnc4160/4170 KO down Sacl; listed in Supplementary Information 6). The DNA fragments were introduced at the both sides of the aphA3 (which confers kanamycin resistant) in pBluescript II SK (+) plasmid. The fragments from the resulted plasmid were introduced 
into $H$. pylori by electroporation.

\section{Construction of non-marker H. pylori mutants}

For constructing non-marker $H$. pylori mutants, ATCC 43504 flaA and cag1 promoter and terminator were cloned into pBluescript SK(+) Smal aphA3

Smal, and sacB gene was cloned into EcoRI site (pKSB plasmid). Mid-log-phase $(\mathrm{OD} 600=0.5-0.7)$ of $H$. pylori in $20 \mathrm{ml}$ culture liquid were washed twice with ice-cold 10\% glycerol and resuspended by $200 \mu \mathrm{l}$ of icecold $10 \%$ glycerol. $1 \mu \mathrm{g}$ of pKSB vector containing aimed mutation and the bacterial liquid were mixed at $4^{\circ} \mathrm{C}$ and electroporated by Micropulser (Bio-Rad) with Ec2 $(2.5 \mathrm{kV})$ setting. After 4 hours incubation at $37^{\circ} \mathrm{C}$ in microaerophilic condition, cells were plated on $5 \%$ sheep blood agar plate TSAll containing 4 $\mu \mathrm{g} / \mathrm{ml}$ Kanamycin and incubated $2-3$ days at $37^{\circ} \mathrm{C}$ under the microaerophilic condition. 4 single colonies were seeded on new 5\% sheep blood agar plate TSAll supplemented with $4 \mu \mathrm{g} / \mathrm{ml}$ Kanamycin and incubated for additional 2 days. Each colony was picked up and were cultured in Brucella broth containing $5 \% \mathrm{FBS}$ at $37^{\circ} \mathrm{C}$ under the microaerophilic condition until $H$. pylori were grown to mid-log phase. $100 \mu$ l of the medium were plated on $5 \%$ sheep blood agar plate supplemented with $2.5 \%$ sucrose and cultured for 2 days. Each colony was seeded on a new $5 \%$ sheep blood agar plate without antibiotics and incubated for 2 days. At the same time, the colony was seeded on a different agar plate with $4 \mu \mathrm{g} / \mathrm{ml}$ Kanamycin to confirm the Kanamycin resistant was disappeared. Grown $H$. pylori were transferred to liquid culture and the genome sequence was confirmed by Sanger sequencing.

\section{Construction of point mutated $H$. pylori}

The $H$. pylori recombination plasmids to establish various mutant strains (T15mut, T16mut, T17mut, T18mut, T19mut) in the upstream region of 
hpnc4160 were constructed by PCR using $H$. pylori genome DNA from the strains isolated from gerbil after 8 weeks as a template, and primers (pKSBHPnc4160 Point mut Apal and pKSB-HPnc4160 Point mut Xhol; listed in Supplementary Information 6), then, the resulted DNA fragments were cloned into suicide pKSB plasmid.

H. pylori T15mut, T16mut, T17mut, T18mut and T19mut mutants were established by introducing each pKSB-based plasmid into H. pylori ATCC 43504 strain.

\section{Construction of NB-cagA-expressing H. pylori}

Based on the full length cagA cDNA sequence of ATCC 43504, we designed HPnc4160-unbound cagA gene sequence (NB-cagA, Extended Data Fig. 5g and $\mathrm{h}$ ). The NB-cagA cDNA were artificially synthesized as pEX-K4J2-cagA mutant of 908 bps (eurofins, 99900008281-1). The cDNA fragments containing mutated $\operatorname{cag} A$ sequence were amplified using primers (pKSB-CagA-NB-Apal, pKSB-CagA-NB-Xhol, listed in Supplemented Information 6), and cloned into a suicide vector pKSB. The resulted plasmids were introduced to $H$. pylori ATCC 43504 to obtain NB-cagA-expressing $H$. pylori.

\section{Construction of hpnc4160 over-expressing H. pylori}

The plasmid for the hpnc4160 overexpressing strain in $H$. pylori was constructed by combination of

DNA fragments of hpnc4160 regions were amplified by PCR using primers (pHel2-4160-de-4170-hed-f Xhol, pHel2-4160-de-4170-hed-r BamHI, Supplemented Information 6) and genome DNA of the ATCC 43504 strain as a template. The resulted DNA fragments included the upstream region of hpnc4160 without including the 5' region of the hpnc4170 region. The DNA was cloned into pHel2 shuttle vector, and introduced into $H$. pylori by 
electroporation.

\section{RNA-seq}

H. pylori were agitation under aerobic conditions and cultured at $37^{\circ} \mathrm{C}$ overnight until the OD value at $600 \mathrm{~nm}$ reached 0.9 . Total RNA from the $H$. pylori were extracted using RNeasy (QIAGEN), according to the manufacturer's instructions. The concentration of total RNA extracted was examined using the NanoDrop Spectrophotometer (ThermoFisher Scientific, Wilmington, DE, USA), according to the manufacturer's instructions. Ten micrograms from each total RNA sample were treated with the MICROBExpress Bacterial mRNA Enrichment kit (Ambion, Grand Island, NY, USA) and RiboMinus ${ }^{\mathrm{TM}}$ Transcriptome Isolation Kit (Bacteria) (Invitrogen, Grand Island, NY, USA) following the manufacturer's instructions. Samples were resuspended in $15 \mu \mathrm{L}$ of RNase-free water. Bacterial mRNAs were chemically fragmented to the size range of $200-250$ bp using $1 \times$ fragmentation solution (Ambion, Grand Island, NY, USA) for $2.5 \mathrm{~min}$ at $94^{\circ} \mathrm{C}$. cDNA was generated according to instructions given in SuperScript Double-Stranded cDNA Synthesis Kit (Invitrogen, Grand Island, NY, USA). Briefly, each mRNA sample was mixed with $100 \mathrm{pmol}$ of random hexamers, incubated at $65^{\circ} \mathrm{C}$ for 5 min, chilled on ice, mixed with $4 \mu \mathrm{L}$ of First-Strand Reaction Buffer (Invitrogen, Grand Island, NY, USA), $2 \mu \mathrm{L}$ of $0.1 \mathrm{M}$ DTT, $1 \mu \mathrm{L}$ of $10 \mathrm{mM}$ RNase-freed NTPmix, $1 \mu \mathrm{L}$ of SuperScript III reverse transcriptase (Invitrogen), and incubated at $50^{\circ} \mathrm{C}$ for $1 \mathrm{~h}$. To generate the second strand, the following Invitrogen reagents were added: $51.5 \mu \mathrm{L}$ of RNase-free water, $20 \mu \mathrm{L}$ of second-strand reaction buffer, $2.5 \mu \mathrm{L}$ of $10 \mathrm{mM}$ RNase-free dNTP mix, $50 \mathrm{U}$. coli DNA Polymerase, $5 \mathrm{U}$ E. coli RNase $\mathrm{H}$, and incubated at $16^{\circ} \mathrm{C}$ for $2.5 \mathrm{~h}$. The Illumina Paired End Sample Prep kit was used for RNA-Seq library creation according to the manufacturer's instructions as follows: Fragmented 
cDNA was end-repaired, ligated to Illumina adaptors, and amplified by 18 cycles of PCR. Paired-end 150-bp reads were generated by high-throughput sequencing with the Illumina Hiseq 2500 Genome Analyzer instrument. After removing the low-quality reads and adaptors, RNA-Seq reads were aligned to the corresponding ATCC 43504 genome using Tophat 2.1.0 (Trapnell et al 2009), allowing for a maximum of two mismatch. If reads mapped to more than one location, only the one showing the highest score was kept. Reads mapping to rRNA and tRNA regions were removed from further analysis. After getting the reads number from every sample, edgeR with TMM normalization method was used to determine the DEGs. Significantly differentially expressed genes (FDR value $<0.05$ and at least two-fold changes) were selected for further analysis.

\section{ITRAQ}

H. pylori ATCC 43504 strains of wild-type, $\Delta h p n c 4160 / h p n c 4170$, and Ahpnc4160/hpnc4170 / pHel2-hpnc4160 were cultured in Brucella Broth containing $5 \%$ FCS to OD600 $=0.9 .1 .5 \mathrm{~mL}$ of each bacterial solution was centrifuged at $5,000 \mathrm{xg}$ for 10 minutes at $4^{\circ} \mathrm{C}$. The pellet was resuspended in Wash buffer (1 M KCl, $15 \mathrm{mM}$ Tris- $\mathrm{HCl}, \mathrm{pH} 7.4$ ), centrifuged again, and the supernatant was removed. The pellet was resuspended in a Wash buffer containing 1 mM AEBSF (4- (2-Aminoethyl) benzenesulfonyl fluoride hydrochloride) and frozen at $-80^{\circ} \mathrm{C}$. iTRAQ analysis was commissioned to Filgen Corporation.

\section{EMSA (electrophoretic mobility shift assay)}

cDNA fragments of small RNA HPnc4160 whole region, the fragments of 150 bp total of each 5'UTR region [from 100 bases upstream from the ribosome binding region (RBS), to 50 bases downstream of the RBS] (hp0410 gene, 
hp0486 gene, horB gene, hp0671 gene, hopE gene, cagA gene, hp1227 gene and helpy_1262 gene), and cDNA of 459 bp total containing the hpnc4160binding 4 region near the 3' tail of the cagA gene, were amplified by PCR using primers (Small RNA HPnc4160 Xhol, Small RNA HPnc4160 EcoRI; HP0410 150 bp Xhol, HP0410 150bp EcoRI; HELPY_0660 150bp Xhol, HELPY_0660 150bp EcoRI; HP0671 150bp Xhol, HP0671 150bp EcoRl; HP0486 150 bp Xhol, HP0486 150bp EcoRI; HPSH_00635 150bp Xhol, HPSH_00635 150bp EcoRI; HPP12_0555 150bp Xhol, HPP12_0555 150bp EcoRI; HP1227 150bp Xhol, HP1227 150bp EcoRI; HELPY_1262 150bp Xhol, HELPY_1262 150bp EcoRI; CagA-B codding Xhol, CagA-B codding EcoRI; listed in Supplementary Information 6) and the ATCC43504 genome as a template. The PCR products were cloned into the position of the downstream of the T7 promoter region of the pBluescript SK (+) plasmid. The NB-cagA mutant RNA used in the gel shift assay was amplified with a T7 promoter by PCR using (T7 promoter CagA-NB EMSA PCR s, T7 promoter CagA-NB EMSA PCR as) as primers and synthesized pEX-K4J2-CagA mutant (eurofins, 99900008281-1) cagA as a template. The $\operatorname{cag} A$ mutant RNA were prepared in the same manner except for mutations in the HPnc4160-binding 4 region. RNA was transcribed from a DNA fragment using an in vitro Transcription T7 kit (TAKARA).

Gel shift assays were performed using 0.04 pmol of 3'-biotin-tagged mRNA with increasing amounts of purified small RNA HPnc4160 in $20 \mu \mathrm{L}$ reactions. Briefly, RNA was denatured $\left(10 \mathrm{~min}, 80^{\circ} \mathrm{C}\right)$ and cooled for $5 \mathrm{~min}$ on ice. Yeast tRNA $1 \mu \mathrm{g}$ (ThermoFisher SCIENTIFIC) was added to the labelled RNA and the reaction was filled up to $10 \mu \mathrm{L}$ with Binding Buffer (10 mM HEPES pH 7.3, 1 $\mathrm{mM} \mathrm{MgCl} 2,20 \mathrm{mM} \mathrm{KCl}, 5 \%$ glycerol). $10 \mu \mathrm{L}$ of either labelled mRNA was added to the HPnc4160. The mixtures were incubated at room temperature for $20 \mathrm{~min}$. Then the samples were mixed with $5 \mu \mathrm{L}$ native loading buffer before loading on a pre-cooled native 6\% poly-acryl amide (PAA), 0.5x TBE gel. Gels 
were run in $0.5 x$ TBE buffer at $30 \mathrm{~mA}$ per gel for 2 hours ${ }^{27}$.

\section{Cleavage assays}

The cDNA of 720 bps of $H$. pylori rnase III was amplified by PCR using primers (pGEX-6P-1 RNasellI Xhol-f, pGEX-6P-1 RNaselll Notl-r, listed in

Supplemented Information 6) and template (genome DNA from ATCC 43504 strain). The cDNA was cloned into pGEX6P-1 vector (GE). E. coli BL21 transformed with the plasmids were subjected to shaking culture in LB broth containing $100 \mu \mathrm{g} / \mathrm{ml}$ ampicillin at $37^{\circ} \mathrm{C}$ with constant shaking at $200 \mathrm{rpm}$. Protein expression was induced with IPTG to a final concentration of $0.1 \mathrm{mM}$, at $4^{\circ} \mathrm{C}$, for 4 hours. The bacteria were collected by centrifugation and the pellets were subjected to GST-fusion protein purification using Glutathione Sepharose 4B (GE) according to the manufacture's instruction. The RNase III protein was excised by PreScission Protease according to the manufacturer's instructions. The purified protein derived from $6.7 \mathrm{~mL}$ of the bacterial culture was developed by SDS-PAGE, and the gel was stained with CBB to confirm that no contaminants were observed in the final product. The protein concentration was determined by absorbance at $280 \mathrm{~nm}$.

Nuclease assays using RNase III was performed using purified $H$. pylori recombinant RNase III. The gel shift assay protocol described above was followed, except that RNase III-specific buffer (25mM Tris pH 7.5, $50 \mathrm{mM} \mathrm{NaCl}$, $50 \mathrm{mM} \mathrm{KCl}, 10$ mM MgCl, 1 mM DTT) was used instead of Binding Buffer. 3'biotin-tagged partial cagA mRNA was incubated on ice with either $5 \mu \mathrm{M}$ of small RNA HPnc4160 for 20 min. RNase III was then added at a final concentration of $300 \mathrm{nM}$ and the reactions were incubated for $1 \mathrm{~min}$ at $37^{\circ} \mathrm{C}$. The samples were mixed with $5 \mu \mathrm{L}$ of native loading buffer before loading on a pre-cooled native 6\% PAA, 0.5x TBE gel ${ }^{28}$. 


\section{ELISA}

AGS cells were co-incubated with $H$. pylori at an MOI of 100 for 12, 24, 36 hours at $37^{\circ} \mathrm{C}$ in a $5 \% \mathrm{CO}_{2}$ environment in 24 well plates. The supernatants were collected and stored at $-30^{\circ} \mathrm{C}$. Enzyme-linked immunosorbent assays (ELISAs) for human IL-8 were performed using the Human IL-8 ELISA Kit (ThermoFisher SCIENTIFIC) according to the manufacturer's instructions. The results are expressed as the means \pm SEM from triplicate experiments.

\section{Immunofluorescence microscopy}

AGS cells were infected with $H$. pylori at an $\mathrm{MOI}$ of 100 for 6 hours at $37^{\circ} \mathrm{C}$ in a $5 \% \mathrm{CO}_{2}$ environment. The cells were fixed with $4 \%(\mathrm{w} / \mathrm{v})$ paraformaldehydePBS at room temperature for $10 \mathrm{~min}$. The cells were then washed with TBS for 3 times, and blocked with Saponin buffer [10\% (v/v) Blocking One (Nakalai, Japan) containing $0.2 \%(\mathrm{w} / \mathrm{v})$ saponin] at $4^{\circ} \mathrm{C}$ for $60 \mathrm{~min}$. Antibodies used for staining were DAPI, Rhodamine Phalloidin (Thermo Fisher SCIENTIFIC, MA, USA), pyCagA. Confocal laser scanning microscopy (CLSM) image acquisition was performed using a Zeiss LSM 800 confocal laser scanning microscope with ZEN 2.3 software (Carl Zeiss, Jena, Germany). 


\section{Extended Data Figures and Tables Legends}

\section{Extended Data Figure 1 | The expression levels of candidate RNAs of} strains recovered from $H$. pylori ATCC43504-infected rodent stomachs. a, The list of mutation rates and expression levels of the candidate RNAs (mRNA or non-coding RNA) of strains recovered from stomachs of gerbils or mice 8 wks post-infection. The Locus tags highlighted in red indicated the candidates common in both of the strains originated from gerbils and mice. N/A, not applicable. $\mathbf{b}$, The expression levels of candidate RNAs (mRNA or non-coding RNA) of isolates recovered from $\mathrm{H}$. pylori ATCC43504-infected C57BL/6 mice stomachs. RNA expression levels of the genes or nearby genes of genome regions (Fig. 1b and Supplementary Information 2), which mutated in $100 \%$ of the recovered strains were assessed.

\section{Extended Data Figure 2 | The length of the poly-T stretches upstream of} the HPnc4160 coding region. a, Schematic diagram of genome DNA sequence around the HPnc4160 and poly-T sequence of genome analyzed strains. Red-colored "T" indicated stretches of poly-T sequence. b, Schematic diagram of genome DNA sequence around the HPnc4160 and poly-T sequence of mice-recovered strains 8 wks after infection ( $n=10$, Hp1 to Hp10). Green indicated HPnc4160 transcribed sequence, red frame indicated poly-T mutated stretches in the upstream region of HPnc4160, and red-colored "T" indicated inserted nucleotides of each recovered strain compared with wildtype. c-e, Time-dependent change in the length of the poly-T stretches upstream of the HPnc4160 coding region in gerbils or mice-recovered strains. c, Strains from Mongolian gerbils infected with ATCC 43504. d, Strains from C57BL/6 mice infected with ATCC 43504. e, Strains from C57BL/6 mice infected with PMSS1. Data are median with interquartile range. $P$ values are 
from non-parametric Dunn's multiple comparison test (at two-sided). ns: not significant. f, Expression levels of HPnc4160 in the recovered $H$. pylori strains from mice $(n=10)$ and $H$. pylori wild-type (T-repeat 14). Relative expression levels of HPnc4160 were measured by real-time PCR and plotted against Trepeat length in the upstream region of HPnc4160. Data are presented as means with s.d. $P$ values represent the results of two-tailed Dunn's multiple comparison test.

\section{Extended Data Figure 3 | Effect of in vitro cultivation on the length of the} poly-T stretches upstream of the HPnc4160 coding region. a, Experimental

strategies schematic. $\mathbf{b}$, The raw data of the DNA sequence analysis of $H$. pylori genomes prepared from original culture (Original \#1 - \#3) and from passaged in vitro for 60 times (60-passaged \#1 - \#3).

\section{Extended Data Figure 4 | The length of the poly-T stretches upstream of} the HPnc4160 coding region and RNA expression levels. a, Growth curves of $H$. pylori ATCC43504 mutants mutated in the number of T repeat in HPnc4160 upstream region. b-c, The relative expression levels of hpnc4170 (b) and HP0811 (c) in the H. pylori strains genetically modified with the Trepeat length. Data are presented as means with s.d. $(n=3) . P$ values are from Dunnett's multiple comparison test (at two-sided). ns: not significant. d, The relative RNA expression levels of target candidates of HPnc4160 showed an inverse correlation with HPnc4160. The total RNA from the indicated H. pylori ATCC 43504 strains were extracted, reverse transcribed, and provided for qPCR to assess the indicated genes. The results represent the average of three separate experiments (each $n=3$ ). Data are presented as means \pm s.d. (error bars). Spearman correlation coefficients ( $r$ ) were used to evaluate the relationships among relative RNA expression of HPnc4160 (Fig. 1h) and each 
target.

\section{Extended Data Figure 5 | Predicted HPnc4160 binding sites. a, Growth} curves of $H$. pylori ATCC43504 mutants. b, Predicted the secondary structure of HPnc4160 RNA by CentroidFold. The bases in the predicted structure are colored according to base-pairing probabilities. Circles in pink and light green color indicated loop structures having probabilities of binding to target RNA sequences. c, Schematic diagram of predicted HPnc4160 binding sites in the corresponding 5'UTR sequence of target genes. Upper sequences indicate target mRNA sequences with base numbers, whereas lower sequences indicate HPnc4160 sequence. Colored sequences are corresponding to the loop structures indicated in (b). d, Binding prediction of HPnc4160 and 5' UTR of $\operatorname{cagA}$ mRNA. d, Schematic diagram of predicted HPnc4160 binding sites in the cagA CM/CRPIA motif of cagA CDS. e-f, Schematic diagram of predicted HPnc4160 binding sites in the corresponding CDS sequence of cagA TYPE 1 (e), TYPE 2 (f), and cagA nonbinding form (NB-cagA) of TYPE 2 (g) and TYPE 1 (h). Upper sequences indicate target cagA mRNA sequences, whereas lower sequences indicate HPnc4160 sequence with base numbers. Colored sequences are corresponding to the loop structures indicated in (b). Mutated nucleotides in cagA mRNA sequence are shown in red. i, Purified RNase III was separated by SDS-PAGE and stained with CBB.

\section{Extended Data Figure 6 | Effect of cagA-NB on host-cell-translocated}

CagA activity. a, Growth curves of $H$. pylori ATCC43504 cagA-NB mutant compared with wild-type. b, Phosphorylated CagA protein levels in cell lysates of AGS cells infected with $H$. pylori ATCC43504. The whole-cell lysates of AGS cells infected with $H$. pylori strains for 6 hours were subjected to western blot against anti-CagA, anti-pY CagA, anti-UreA, and anti-Actin antibodies. The 
band intensities were measured and calculated by Image J software. c, IL-8 production from AGS cells infected with $H$. pylori ATCC43504. The supernatants from AGS cells infected with $H$. pylori strains shown in the figure for the indicated time were subjected to ELISA system for IL-8 production. The results represent the average of three separate experiments (each $n=3$ ). Data are presented as means \pm s.d. (error bars).

\section{Extended Data Figure 7 | Characterization of clinical isolates. a, Clinical} isolates of non-cancer (Non-Cancer, n=39) and cancer (Cancer, n=17) patients, which used in Fig. 4g, showed equal growth rate. The strains cultured on TSAll containing $5 \%$ sheep blood plates for 2 days were inoculated in Brucella broth containing $5 \%$ FCS, adjusted $\mathrm{OD}_{600 \mathrm{~nm}}$ at 0.1 , then cultured in microaerobic condition with agitation for 16 hours. The turbidity of the cultures was assessed at $O D_{600 n m}$. The Data are presented as medians with interquartile range. $P$ values represent the results of the two-tailed MannWhitney test. ns: not significant. $\mathbf{b}$, The relative RNA expression levels of target candidates of HPnc4160. Spearman correlation coefficients ( $r$ ) were used to evaluate the relationships among relative RNA expression of HPnc4160 and each target. c, Comparison of expression levels of mRNA (HELPY_1262 and HP1227) in clinical isolates of non-cancer (NC, n=39) and cancer $(C, n=17)$ patients. The expression levels of mRNA were normalized with the levels of $23 S$ rRNA. Data are presented as medians with interquartile range. $P$ values represent the results of the two-tailed Mann-Whitney test. ns: not significant.

\section{Extended Data Figure 8 | Infection-induced silencing of HPnc4160 upregulates target genes expression and promote bacterial host} adaptation and canceration during chronic $\boldsymbol{H}$. pylori infection. $H$. pylori 
infection in vivo leads elongation of T-stretch in the upstream region of HPnc4160 sRNA coding region, which results in decreased expression levels of sRNA HPnc4160. Gene silencing of HPnc4160 results in increased levels of target genes coding OMPs and CagA, and as a result, the levels of bacterial colonization and CagA translocation into the attached host cells were increased.

\section{Extended Data Table 1 | The list of mutated genome regions in the strains recovered from $\boldsymbol{H}$. pylori-infected rodents' stomachs.}

The list showed the genome regions that mutated in the strains isolated from the stomachs of rodents 8 weeks post infection. The DNA sequences in the regions listed in the table were mutated in more than $50 \%$ of the 40 strains recovered from Mongolian gerbils, or, in all of the 10 strains recovered from C57BL/6 mice. N/A, not applicable.

\section{Extended Data Table 2 | Comparative analysis of expression levels between $H$. pylori ATCC43504 $\Delta$ hpnc4160/hpnc4170 mutant and wild-type strains.}

a, Comparative analysis of RNA expression levels between $H$. pylori ATCC $43504 \Delta h p n c 4160 / h p n c 4170$ mutant and wild-type strains by RNA-seq.

\section{Footnote |}

Normalized expression level and fold change of the strains were listed. Genes with $P$-values by Empirical Analysis of Digital Gene Expression in R (edgeR) test showed less than 0.001 were listed (17 factors). Eight genes selected by RNA-seq and iTRAQ analysis (Fig. 2c) were highlighted in red.

b, Comparative analysis of protein expression levels between $H$. pylori ATCC43504 $\Delta$ hpnc4160/hpnc4170 mutant and wild-type strains by iTRAQ. 
Proteins showing relative protein abundance with $P$-value of less than 0.01 were listed ( 21 factors). Eight proteins selected by RNA-seq and ITRAQ analysis (Fig. 2c) were highlighted in red. 


\section{Supplementary Information Legends}

Supplementary information 1 | Summary of mutations in the isolates recovered from $H$. pylori-infected Mongolian gerbils.

The number of mutations in the isolates of 40 strains recovered from $\mathrm{H}$. pyloriinfected Mongolian gerbils' stomachs 8 weeks after post-infection were listed.

Supplementary information 2 | The mutated sequence list of 40 strains recovered from $H$. pylori-infected Mongolian gerbils' stomach.

Supplementary information 3 | Summary of mutations in the isolates recovered from $H$. pylori-infected C57BL/6 mice. The number of mutations in the isolates of 10 strains recovered from $\mathrm{H}$. pylori-infected mice stomachs 8 weeks after post-infection were listed.

Supplementary information 4 | The mutated sequence list of 10 strains recovered from $H$. pylori-infected C57BL/6 mice stomach.

Supplementary information 5 | Information of $H$. pylori clinical isolates used in Fig. 1f, and Fig 4g.

Supplementary information 6 | Primers used in this study. 


\section{Acknowledgements}

The authors would like to thank Manuel Amieva for providing us $H$. pylori strains. We gratefully acknowledge Keisuke Katsura for his support. We would like to thank the members of the Division of Bacteriology, Department of Infectious Diseases Control, International Research Center for Infectious Diseases, The Institute of Medical Science, The University of Tokyo, and the members of Department of Infection Microbiology, Research Institute for Microbial Diseases, Osaka University. This work was supported in part by Grant-in-Aid for Scientific Research from the Ministry of Education, Culture, Sports, Science, and Technology of Japan [17K19551, 18K07127, 19K22704 (to H.M.), 16K07083 (to T.S.), 17K14974 (to M.T.)], the Naito Foundation, the Tokyo Biochemical Research Foundation (to H.M. and P.S.), and the Kao Foundation for Arts and Sciences (to K.K.). This work was supported by MEXT KAKENHI (No. 221S0002).

\section{Details of Author Contributions}

Conceived and designed the experiments: RKD, KK, HM; performed the experiments: RKD, KK, RO, YO, TS, TO, TI, PS; analyzed the data RKD, KK, YO, ZB, RY, KN, TH, HM; contributed reagents/materials/analysis tools: RKD, KK, TS, TVP, EK, AH, MT, AS, HA, TTB, LTN, KVV, DQDH, TS, YY, HM; prepared the manuscript: RKD, KK, TH, HM. 
bioRxiv preprint doi: https://doi.org/10.1101/2020.02.15.950279; this version posted February 21, 2020. The copyright holder for this

preprint (which was not certified by peer review) is the author/funder. All rights reserved. No reuse allowed without permission. 


\section{References}

1. Hatakeyama, M. Helicobacter pylori CagA and Gastric Cancer: A Paradigm for Hit-and-Run Carcinogenesis. Cell Host Microbe 15, 306316 (2014).

2. Kuipers, E.J., Pérez-Pérez, G.I., Meuwissen, S.G. \& Blaser, M. J. Helicobacter pylori and atrophic gastritis: importance of the cagA status. J NatI Cancer / 87, 1777-80 (1995).

3. Blaser, M. et al. Infection with Helicobacter pylori strains possessing cagA is associated with an increased risk of developing adenocarcinoma of the stomach. Cancer Res 55, 2111-5 (1995).

4. Mimuro, H. et al. Grb2 Is a Key Mediator of Helicobacter pylori CagA Protein Activities. Mol Cell 10, 745-755 (2002).

5. Viala, J. et al. Nod1 responds to peptidoglycan delivered by the Helicobacter pylori cag pathogenicity island. Nat Immunol 5, 11661174 (2004).

6. Mimuro, H. et al. Helicobacter pylori dampens gut epithelial self-renewal by inhibiting apoptosis, a bacterial strategy to enhance colonization of the stomach. Cell Host Microbe 2, 250263 (2007).

7. Suzuki, M. et al. Helicobacter pylori CagA phosphorylation-independent function in epithelial proliferation and inflammation. Cell Host Microbe 5, 2334 (2009).

8. Backert, S. \& Naumann, M. What a disorder: proinflammatory signaling pathways induced by Helicobacter pylori. Trends Microbiol 18, 479486 (2010). 
9. Zimmermann, S. et al. ALPK1- and TIFA-Dependent Innate Immune Response Triggered by the Helicobacter pylori Type IV Secretion System. Cell Reports 20, 23842395 (2017).

10. Gall, A., Gaudet, R. G., Gray-Owen, S. D. \& Salama, N. R. TIFA Signaling in Gastric Epithelial Cells Initiates the cag Type 4 Secretion System-Dependent Innate Immune Response to Helicobacter pylori Infection. Mbio 8, (2017).

11. Stein, S. C. et al. Helicobacter pylori modulates host cell responses by CagT4SS-dependent translocation of an intermediate metabolite of LPS inner core heptose biosynthesis. Plos Pathog 13, e1006514 32 (2017).

12. Suerbaum, S. \& Josenhans, C. Helicobacter pylori evolution and phenotypic diversification in a changing host. Nat Rev Microbiol 5, 441452 (2007).

13. Yamaoka, Y. et al. Helicobacter pylori outer membrane proteins and gastroduodenal disease. Gut 55, 775781 (2006).

14. Morelli, G. et al. Microevolution of Helicobacter pylori during Prolonged Infection of Single Hosts and within Families. Plos Genet 6, e1001036 (2010).

15. Kennemann, L. et al. Helicobacter pylori genome evolution during human infection. Proc National Acad Sci 108, (2011).

16. Dutta, T. \& Srivastava, S. Small RNA-mediated regulation in bacteria: A growing palette of diverse mechanisms. Gene 656, 60-72 (2018). 
17. Vannini, A. et al. Comprehensive mapping of the Helicobacter pylori NikR regulon provides new insights in bacterial nickel responses. Sci Rep-uk 7, 45458 (2017).

18. Sharma, C. M. et al. The primary transcriptome of the major human pathogen Helicobacter pylori. Nature 464, 250-255 (2010).

19. Miyakoshi, M., Chao, Y. \& Vogel, J. Regulatory small RNAs from the 3' regions of bacterial mRNAs. Curr Opin Microbiol 24, 132-139 (2015).

20. Saadat, I. et al. Helicobacter pylori CagA targets PAR1/MARK kinase to disrupt epithelial cell polarity. Nature 447, 330333 (2007).

21. Salaün, L., Ayraud, S. \& Saunders, N. J. Phase variation mediated niche adaptation during prolonged experimental murine infection with Helicobacter pylori. Microbiology+151, 917923 (2005).

22. Deitsch, K. W., Lukehart, S. A. \& Stringer, J. R. Common strategies for antigenic variation by bacterial, fungal and protozoan pathogens. Nat Rev Microbiol 7, 493-503 (2009).

23. Konno, M. et al. Predominance of Mother-to-Child Transmission of Helicobacter pylori Infection Detected by Random Amplified Polymorphic DNA Fingerprinting Analysis in Japanese Families. Pediatric Infect Dis J 27, 999 1003 (2008).

24. Linz, B. et al. A mutation burst during the acute phase of Helicobacter 
pylori infection in humans and rhesus macaques. Nat Commun 5, 18 (1AD).

25. Hirukawa, S. et al. Characterization of morphological conversion of Helicobacter pylori under anaerobic conditions. Microbiol Immunol 62, 221228 (2018).

26. Park, J., Forman, D., Waskito, L., Yamaoka, Y. \& Crabtree, J. E. Epidemiology of Helicobacter pylori and CagA-Positive Infections and Global Variations in Gastric Cancer. Toxins 10, 163 (2018).

27. Pernitzsch, S., Tirier, S., Beier, D. \& Sharma, C. A variable homopolymeric G-repeat defines small RNA-mediated posttranscriptional regulation of a chemotaxis receptor in Helicobacter pylori. Proc National Acad Sci 111, E501 E510 (2014).

28. Michaux, C. et al. RNA target profiles direct the discovery of virulence functions for the cold-shock proteins CspC and CspE. Proc National Acad Sci 114, 6824-6829 (2017). 
bioRxiv preprint doi: https://doi.org/10.1101/2020.02.15.950279; this version posted February 21, 2020. The copyright holder for this

preprint (which was not certified by peer review) is the author/funder. All rights reserved. No reuse allowed without permission.
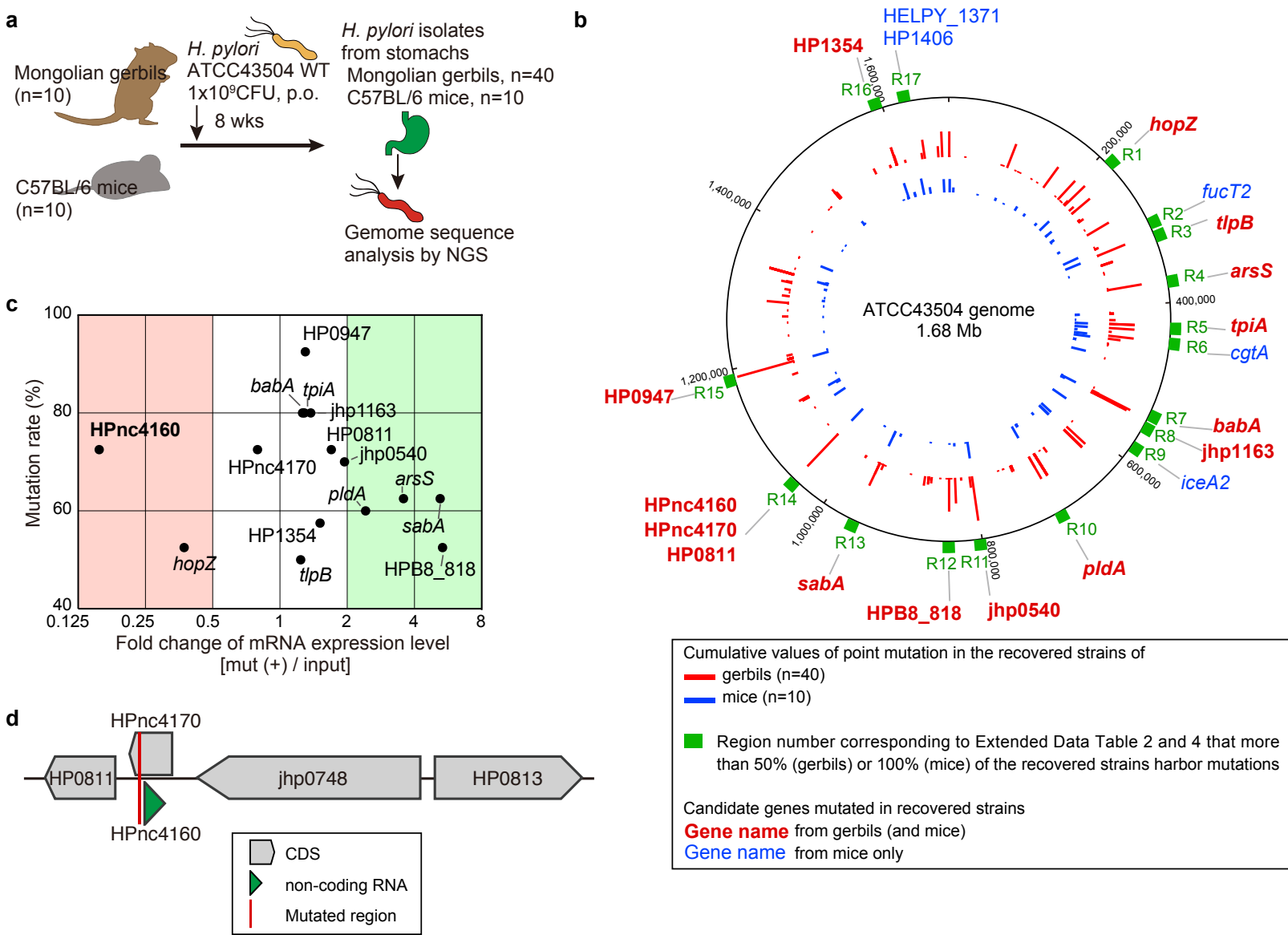

Cumulative values of point mutation in the recovered strains of
gerbils $(n=40)$
mice $(n=10)$
Region number corresponding to Extended Data Table 2 and 4 that more
than $50 \%$ (gerbils) or $100 \%$ (mice) of the recovered strains harbor mutations
Candidate genes mutated in recovered strains
Gene name from gerbils (and mice)
Gene name from mice only

e $\operatorname{ATCC} 43504$ WT

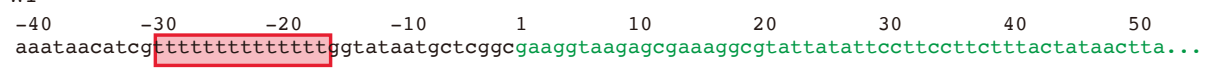

Poly-T elongation pattern in the recovered strains

No. of strains/total

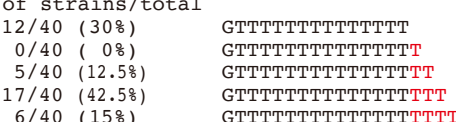
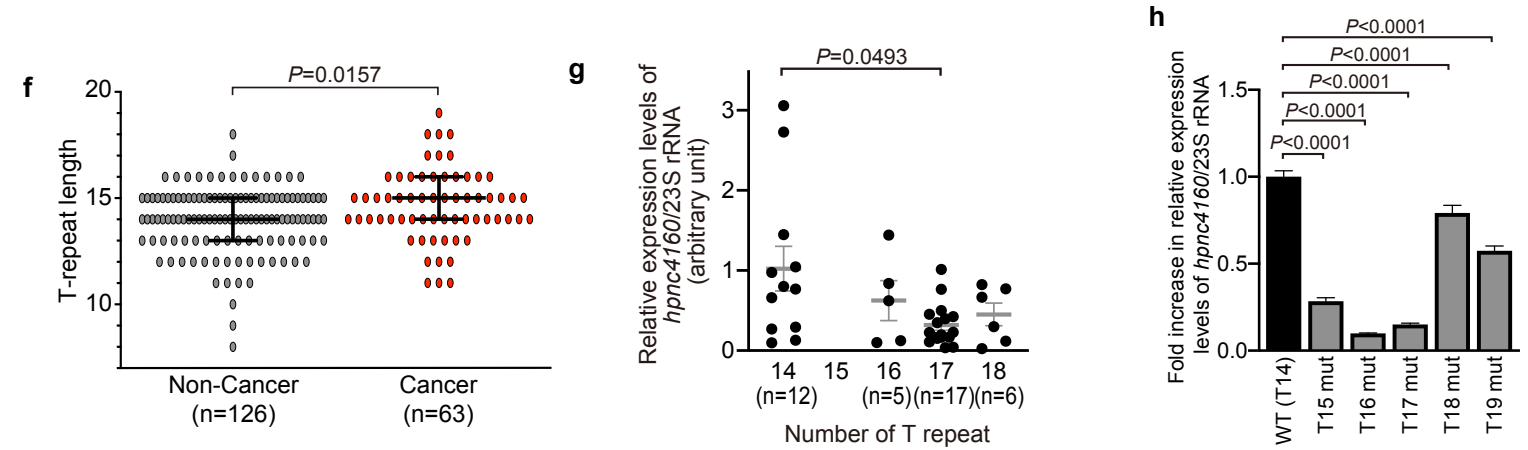

Fig. 1 Kinoshita et al. 

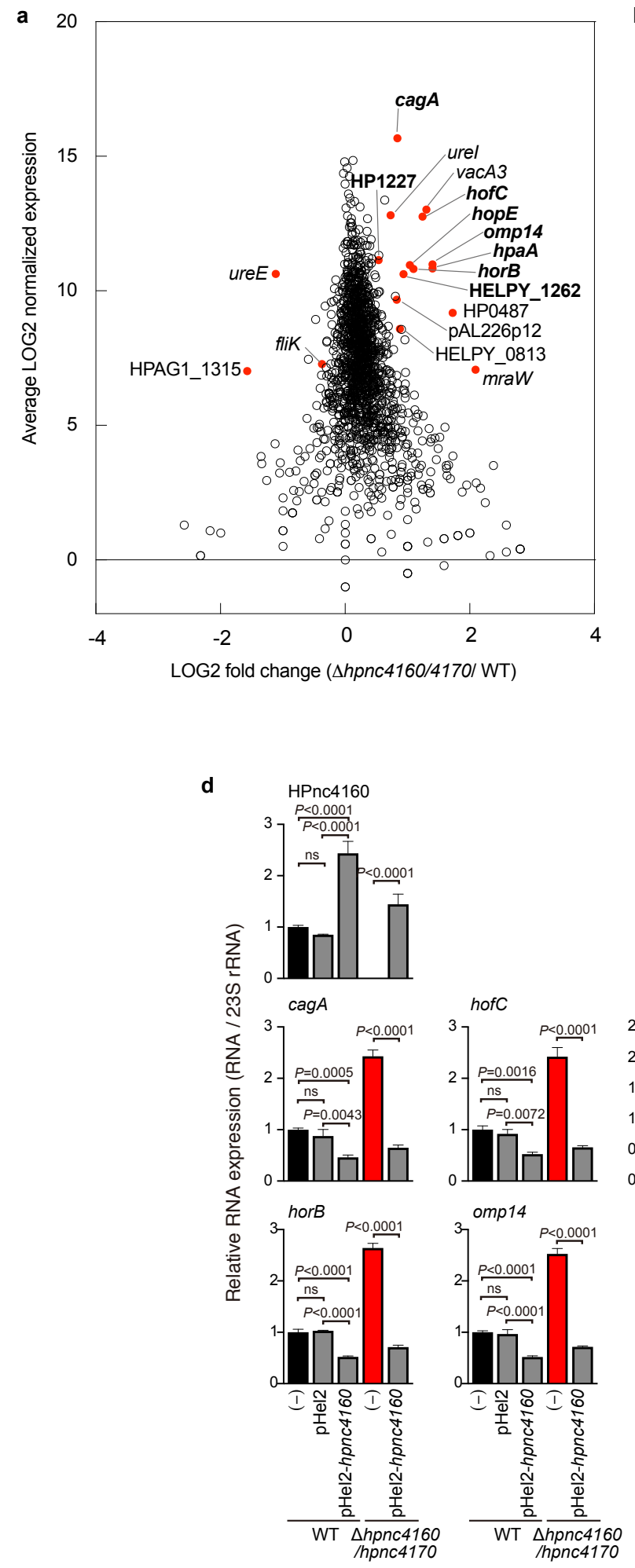

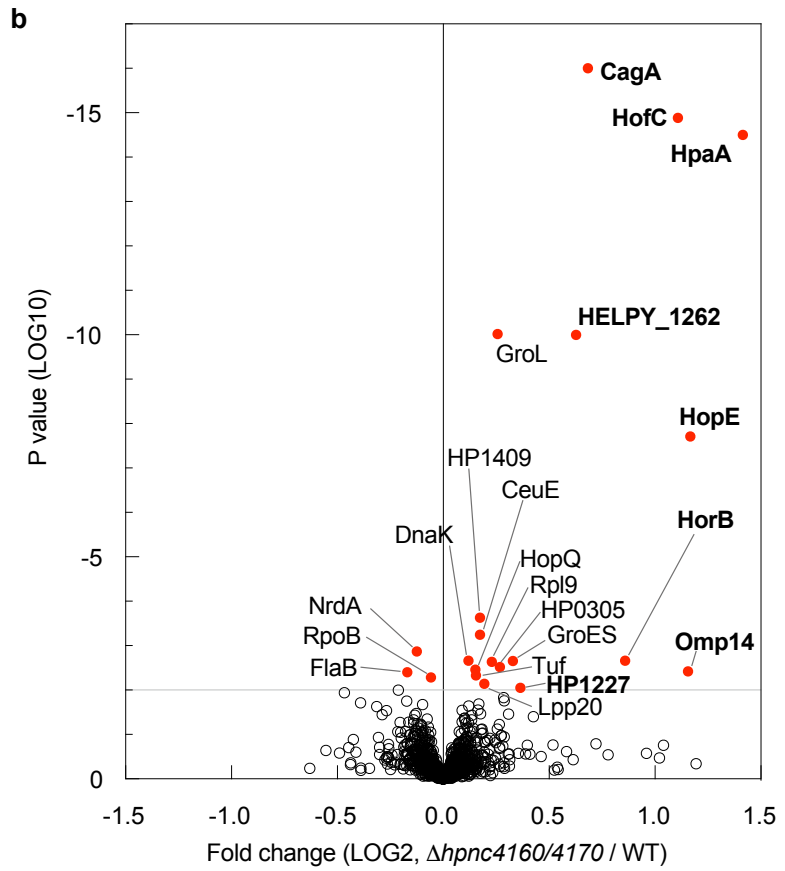

c
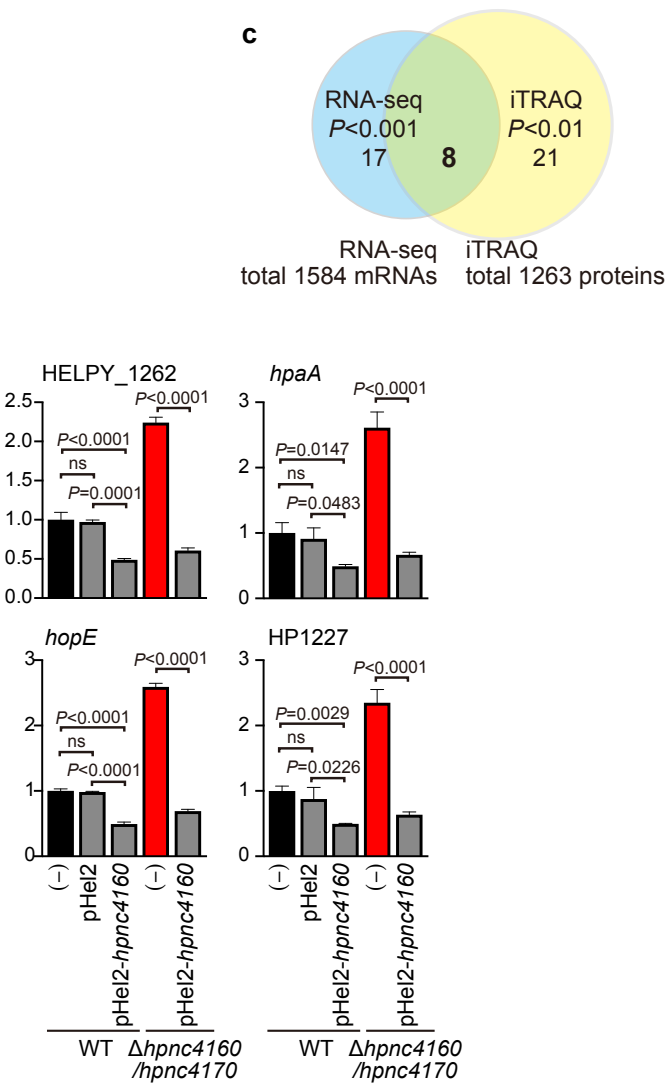
a
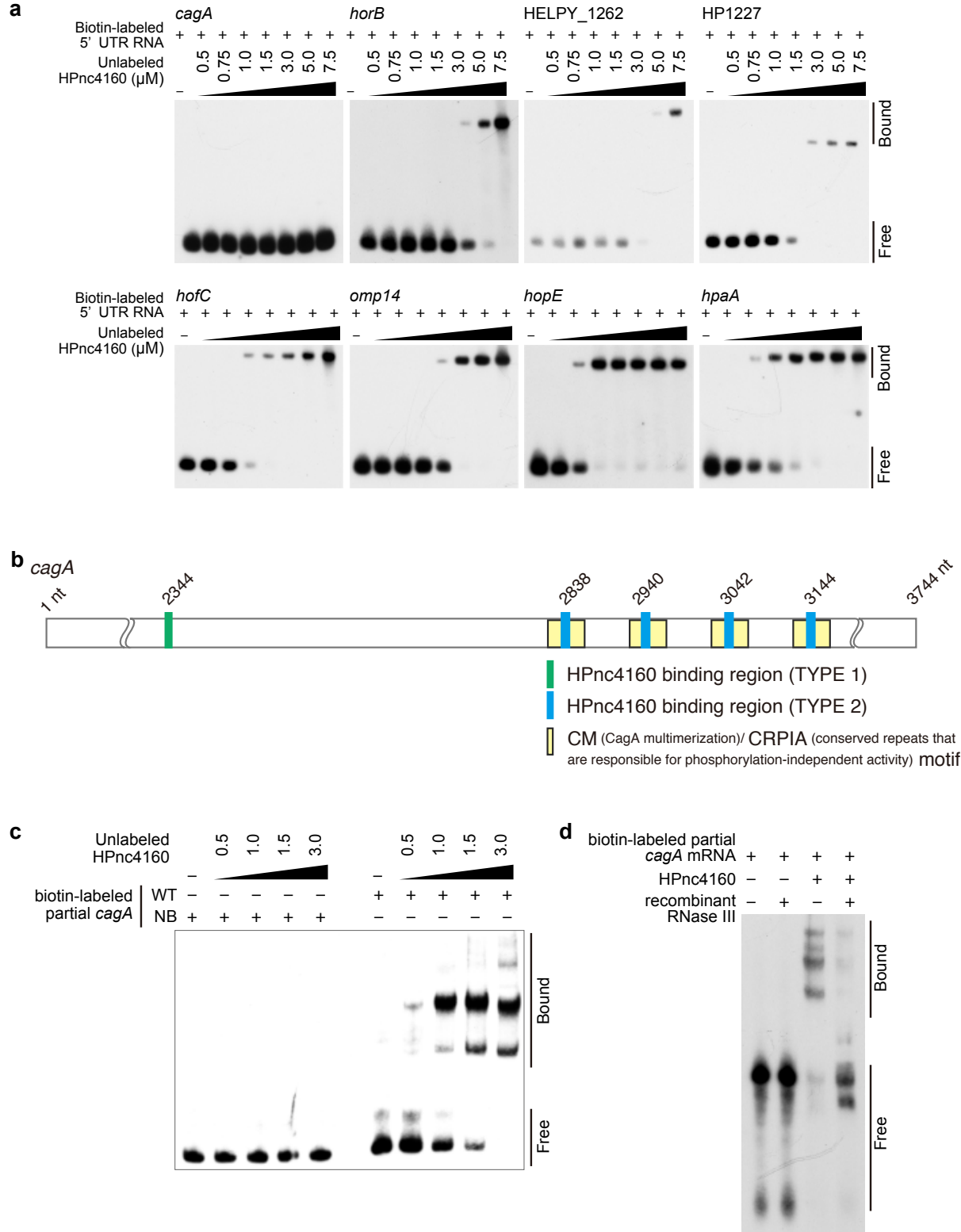

Fig. 3 Kinoshita et al. 
a
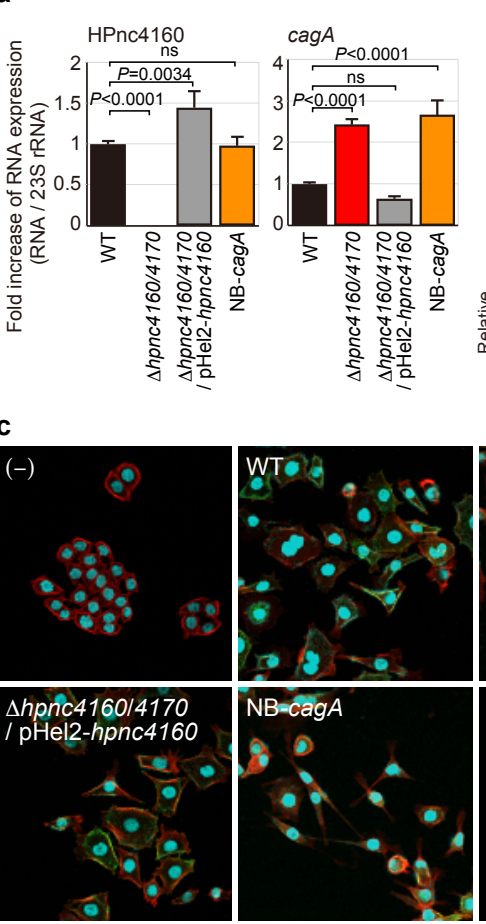

e
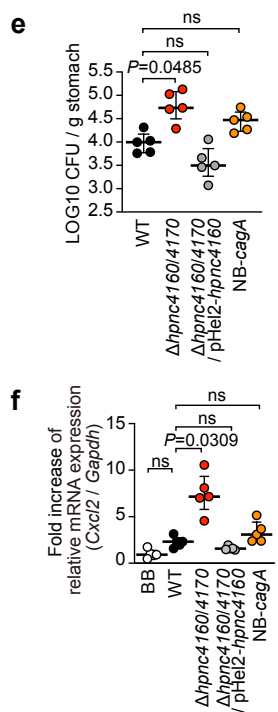
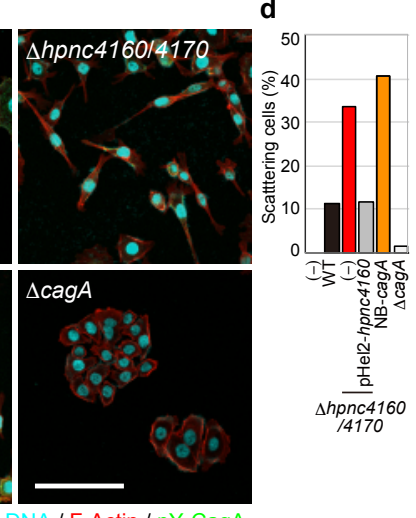

b

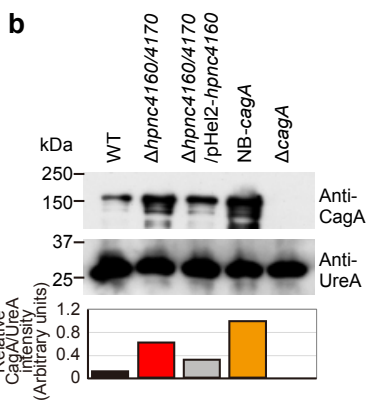

/ F-Actin / pY-CagA
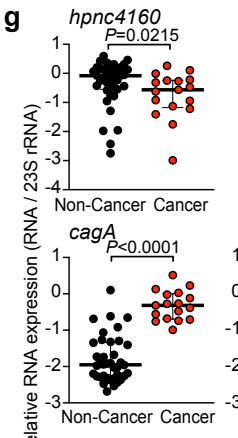

hor $=0.0004$
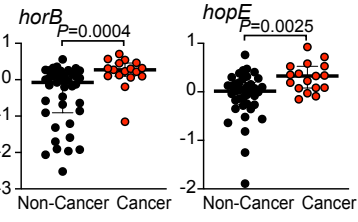

hofC

2 $\stackrel{ }{h p a=0.0205}$
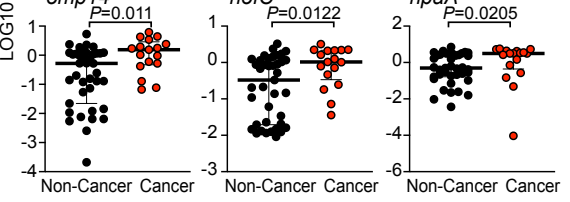

omp 14 
bioRxiv preprint doi: https://doi.org/10.1101/2020.02.15.950279; this version posted February 21, 2020. The copyright holder for this

preprint (which was not certified by peer review) is the author/funder. All rights reserved. No reuse allowed without permission.

a

\begin{tabular}{|c|c|c|c|c|c|c|}
\hline \multirow[t]{2}{*}{$\begin{array}{l}\text { Region } \\
\text { No. }\end{array}$} & \multirow[t]{2}{*}{ Gene symbol } & \multirow[t]{2}{*}{ Gene description } & \multicolumn{2}{|c|}{$\begin{array}{c}\text { Mongolian gerbils } \\
\text { (8 wks post infection) }\end{array}$} & \multicolumn{2}{|c|}{$\begin{array}{c}\text { C57BL/6 mice } \\
\text { (8 wks post infection) }\end{array}$} \\
\hline & & & $\begin{array}{c}\text { Fold change } \\
\text { of mRNA } \\
\text { expression } \\
\text { level } \\
\text { [mut(+)/input] }\end{array}$ & $\begin{array}{l}\text { Mutation rate } \\
(\%, / 40 \text { strains })\end{array}$ & $\begin{array}{c}\text { Fold change } \\
\text { of mRNA } \\
\text { expression } \\
\text { level } \\
\text { [mut(+)/input] }\end{array}$ & $\begin{array}{c}\text { Mutation rate } \\
(\%, / 10 \text { strains })\end{array}$ \\
\hline R1 & hopZ & outer membrane protein & 0.373 & 52.5 & $\mathrm{~N} / \mathrm{A}$ & $\mathrm{N} / \mathrm{A}$ \\
\hline $\mathrm{R} 2$ & fuct2 & alpha-1,2-fucosyltransferase & N/A & N/A & 0.758 & 100.0 \\
\hline R3 & $\operatorname{tlpB}$ & methyl-accepting chemotaxis protein & 1.239 & 50.0 & 0.913 & 100.0 \\
\hline $\mathrm{R} 4$ & arss & histidine kinase sensor protein & 3.572 & 62.5 & N/A & N/A \\
\hline R5 & tpiA & triosephosphate isomerase & 1.286 & 80.0 & 0.825 & 100.0 \\
\hline R6 & $\operatorname{cgt} A$ & beta-1,4-N-acetylgalactosamyltransferase & N/A & N/A & 1.453 & 100.0 \\
\hline R7 & $b a b A$ & outer membrane protein babA & 1.263 & 80.0 & 1.243 & 100.0 \\
\hline R8 & jhp1163 & hypothetical protein & 1.374 & 80.0 & N/A & N/A \\
\hline R9 & iceA2 & Ulcer-associated gene restriction endonuclease & N/A & N/A & 1.104 & 100.0 \\
\hline R10 & pldA & phospholipase A1 & 2.420 & 60.0 & N/A & N/A \\
\hline $\mathrm{R} 11$ & jhp0540 & hypothetical protein & 1.946 & 70.0 & 1.497 & 100.0 \\
\hline $\mathrm{R} 12$ & HPB8_818 & family 25 glycosyl transferase & 5.346 & 52.5 & N/A & N/A \\
\hline R13 & $s a b A$ & outer membrane protein sabA & 5.215 & 62.5 & 1.512 & 100.0 \\
\hline R14 & HPnc4160 & mRNA/antisense RNA family IsoB & 0.155 & 72.5 & 0.297 & 100.0 \\
\hline R14 & HPnc4170 & mRNA/antisense RNA family aapB & 0.794 & 72.5 & 1.043 & 100.0 \\
\hline R14 & HP0811 & hypothetical protein & 1.698 & 72.5 & 1.075 & 100.0 \\
\hline R15 & HP0947 & hypothetical protein & 1.300 & 92.5 & 0.511 & 100.0 \\
\hline R16 & HP1354 & adenine-specific DNA methyltransferase & 1.512 & 57.5 & 1.138 & 100.0 \\
\hline $\mathrm{R} 17$ & HELPY_1371 & Type III restriction enzyme R protein & N/A & N/A & 1.018 & 100.0 \\
\hline $\mathrm{R} 17$ & HP1406 & biotin synthase & N/A & N/A & 1.200 & 100.0 \\
\hline
\end{tabular}

b

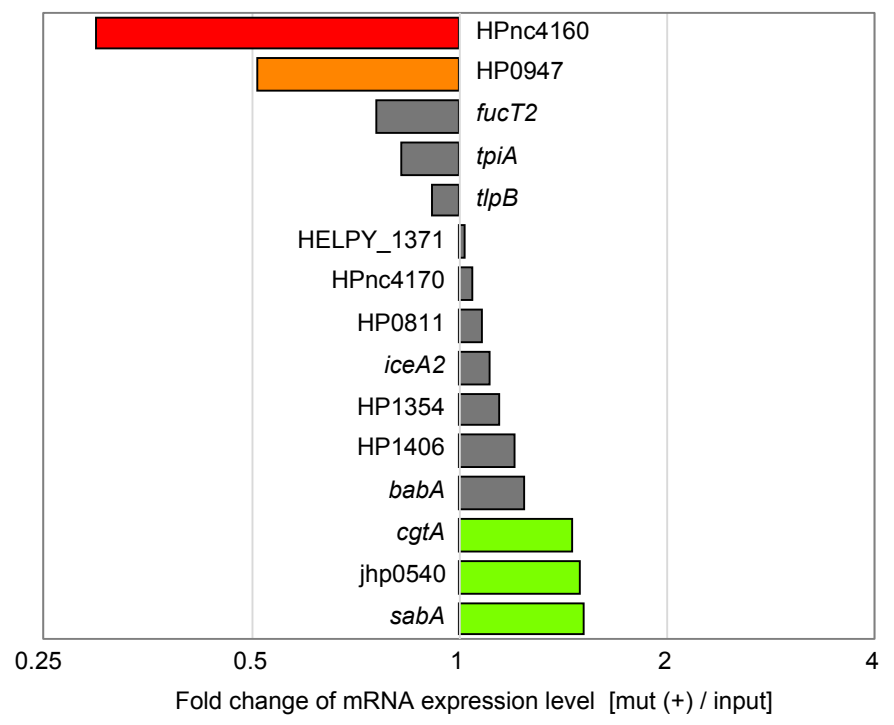


a

H. pylori ATCC4350

H. pylori PMSS1

H. pylori SSI

H. pylori 26695

H. pylori J99

H. pylori HPAG

H. pylori G27

H. pylori Shi470

H. acinonychis Sheeba

H. pylori ATCC43504

H. pylori PMSS1

H. pylori SS1

H. pylori 26695

H. pylori J99

H. pylori HPAG1

H. pylori G27

pylori Shi470

H. acinonychis Sheeba
$-40 \quad-30 \quad-20$

$-10$

1

10

20

30

40

(1) aataacatc-tttttttttttt-------ggtataatgc-cgcctaaggtaagagcgaaaggcgtattata----ttccttccttctt aaataacatc-ttttttttttttt------ggtataatgc-cgcctaaggtaagagcgaaaggcgtattata----ttccttccttctt aaataacatcgtttttttttttttt------ggtataatgctcggcgaaggtaagagcgaaaggcgtattata----ttccttctttctt aaataacatcgtttttttttttttt-----ggtataatgctcggcgaaggtaagagcgaaaggcgtattata----ttccttccttctt aaataacatcgttttttttttttttttt--ggtataatgctcggcgaaggtaagagcgaaaggcgtattata----ttccttccttctt aaataacatc-tttttttttttttttt---ggtataatgc-cgcttaaggtaagagcgaaaggcgtattata----ttccttccttctt aaataacatcgtttttttttttttt-----ggtataatgctcggtgaaggtaagagcaaaaggcgtattatattccttccttccttctt aaataaca---tttttttttttagtataatagtataatgt-tgttg--ggtaagggcaaagggcg-aaaata----ttccttccttctt

41 50 60 70 80 90 100 tactaac-ttagca-ttttaatcaacttttt-------cattaaaatgtcctgacgctcttacctt-aa tactataac-ttagca-ttttaatcaacttttt-------cattaaaatgtcctgacgctcttaccttttc tactataac-ttagca-ttttaatcaacttttt-------cattaaatgtcctgacgctcttaccttttc tactataac-ttagca-ttttaatcaacttttt-------cattaaaatgtcctgacgctcttacctt-aa tactataac-ttagca-ttttaatcaacttttt-------cattaaaatgtcctgacgctcttaccttaaa tactataac-ttagca-ttttaatcaacttttt-------cattaaaatgtcctgacgctcttacctt-aa tactataac-ttagca-ttttaatcaacttttt-------cattaaaatgtcctgacgctcttacctt--tactataac-ttagca-ttttaataaacttttt-------cattaaaatgtcctgacgctcttaccttcaa tactataacattagcatttttagtaaactttttctttttacattaaaatgtcctaatgctcttacctt--a

b

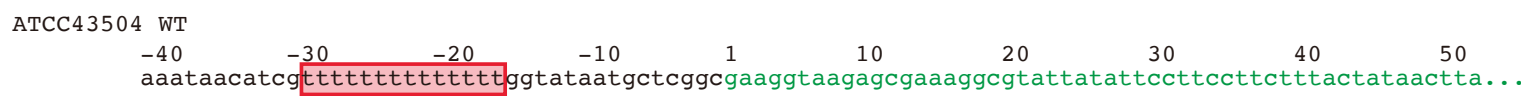

Poly-T elongation pattern in the recovered strains from mice

No. of strains/total

$$
\begin{aligned}
& 1 / 10(10 \%) \text { GTTTTTTTTTTTTTTT } \\
& \text { GTTTTTTTTTTTTTT } \\
& 4 / 40 \text { (40\%) GTTTTTTTTTTTTTTTTT }
\end{aligned}
$$
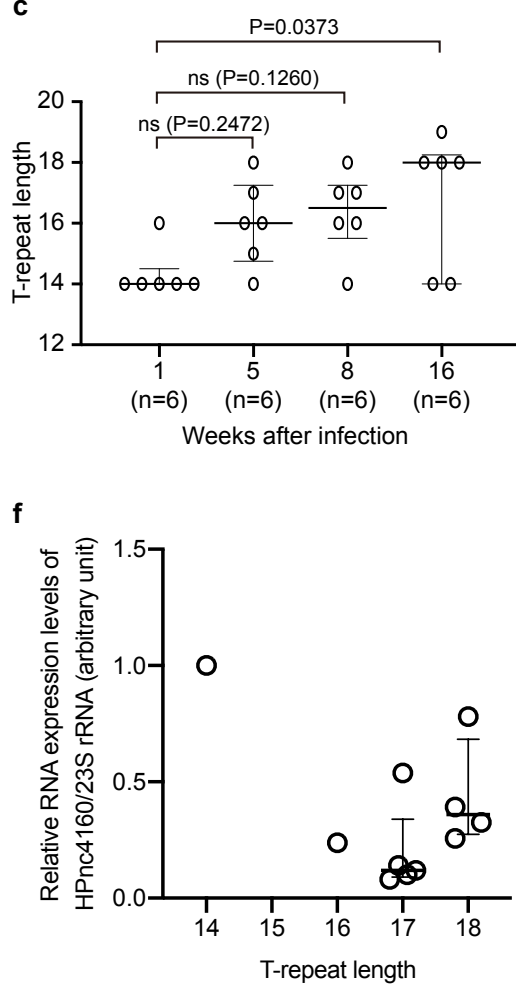

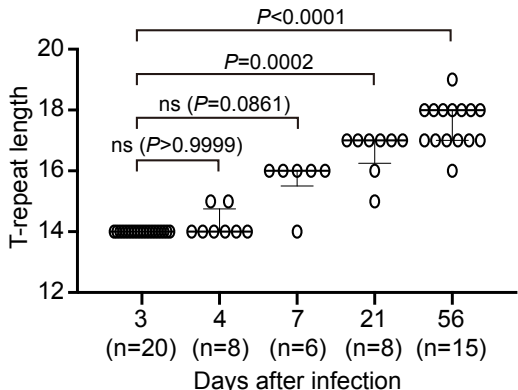

Days after infection

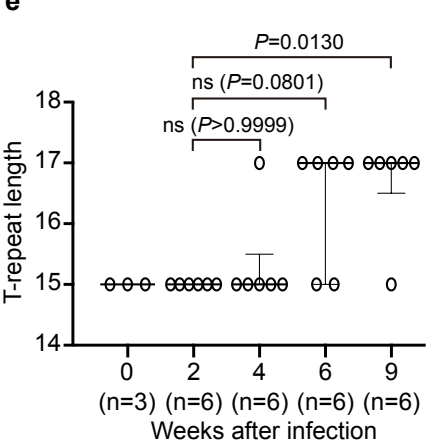


bioRxiv preprint doi: https://doi.org/10.1101/2020.02.15.950279; this version posted February 21, 2020. The copyright holder for this

preprint (which was not certified by peer review) is the author/funder. All rights reserved. No reuse allowed without permission.

a

ATCC43504 WT

- Inoculation on TSAll agar plate

$\downarrow$ - Incutbation at $37^{\circ} \mathrm{C}$ in microaerobic condition for 2 days

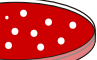

- Suspending collected bacteria into broth

$\downarrow$ and adjusting OD600nm to 0.05
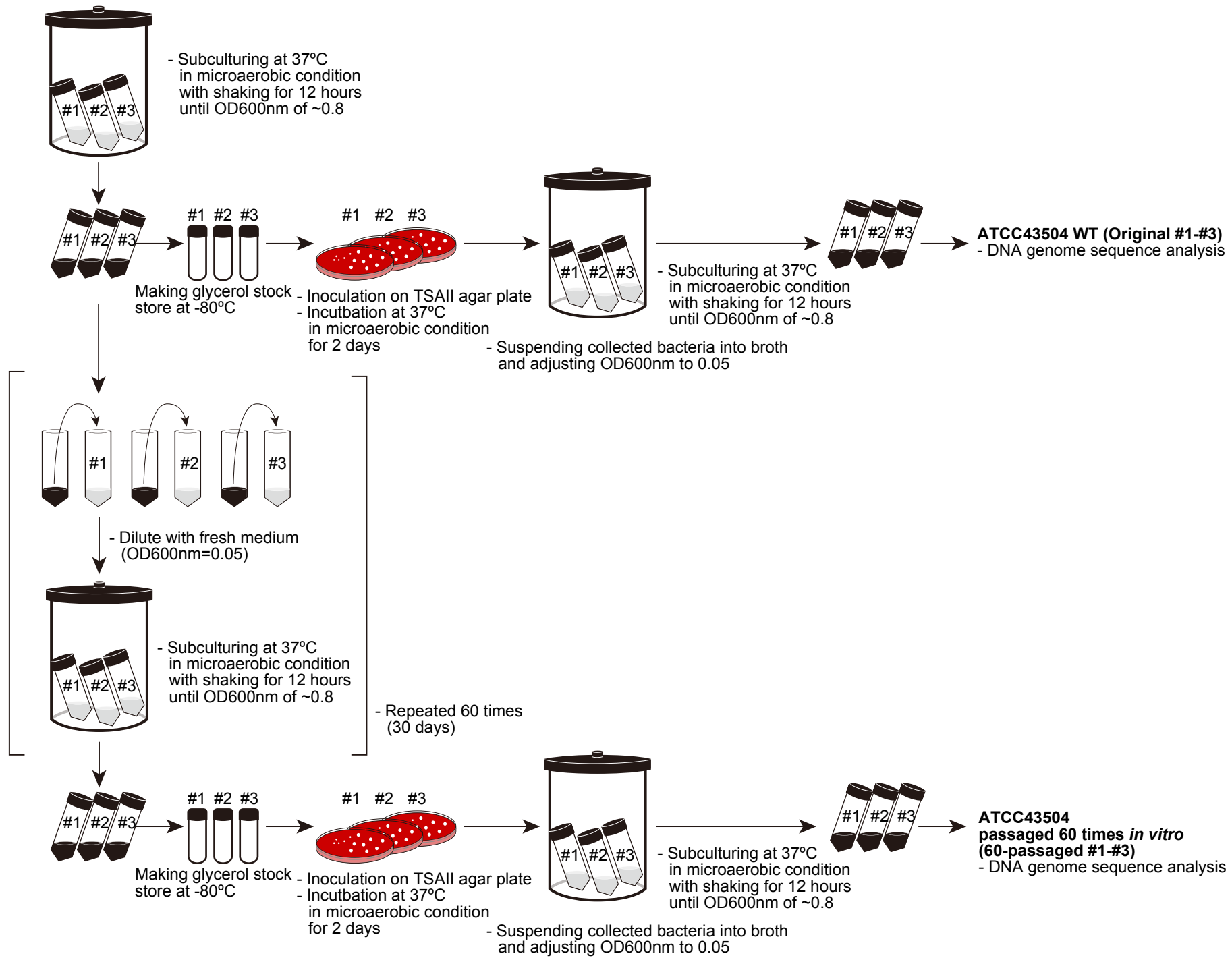

ATCC43504

passaged 60 times in vitro

(60-passaged \#1-\#3)

DNA genome sequence analysis

Making glycerol stock Incutbation at $37^{\circ} \mathrm{C}$

for 2 days

Suspending collected bacteria into broth and adjusting OD600nm to 0.05

b

Original \#1

A A TAACATCGTTTTTTTTTTTTTGGTATAA

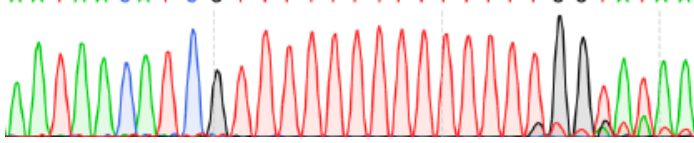

Original \#2

A A T C G T T T T T T T T T T T T T T G G T A T A A

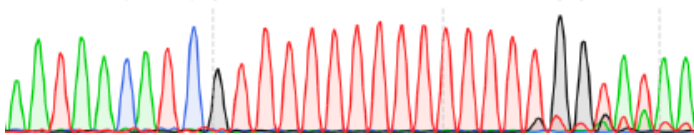

Original \#3

A A T A A CAT C GTTTTTTTTTTTT T G TATAA

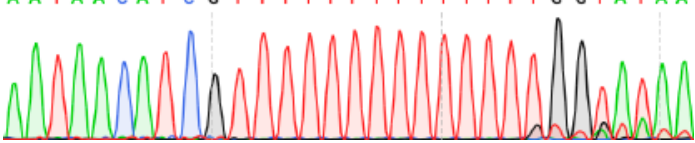

60-passaged \#1

A A T A A CATCG TTTTTTTTTTTTTGGTATAA

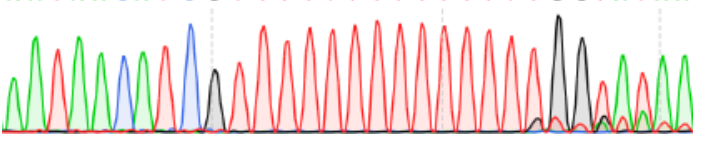

60-passaged \#2

A A T A A CA T C G T T T T T T T T T T T T T T G G TATA A

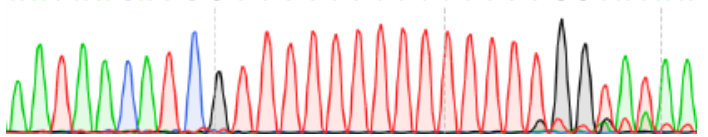

60-passaged \#3

A A T A A C A T C G T T T T T T T T T T T T T T G G T A TA A

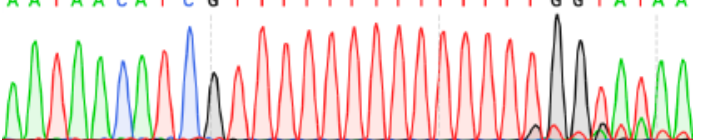


a

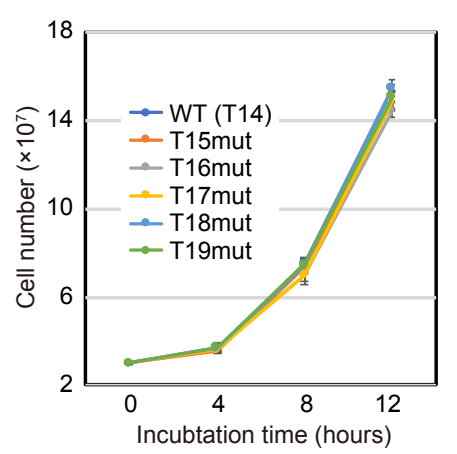

b

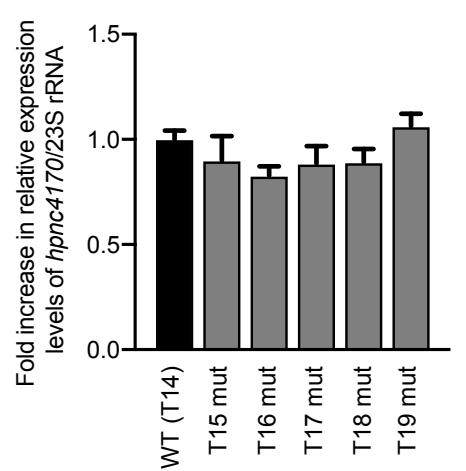

\section{c}

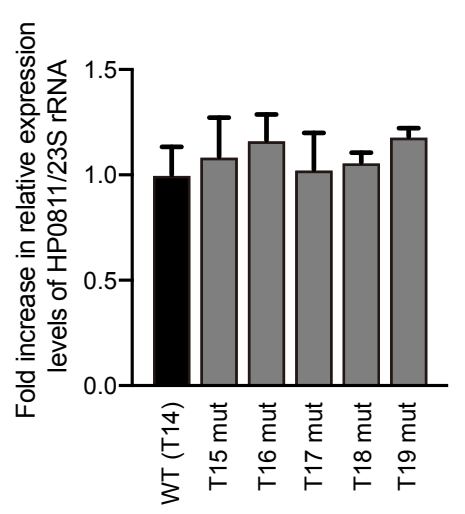

d
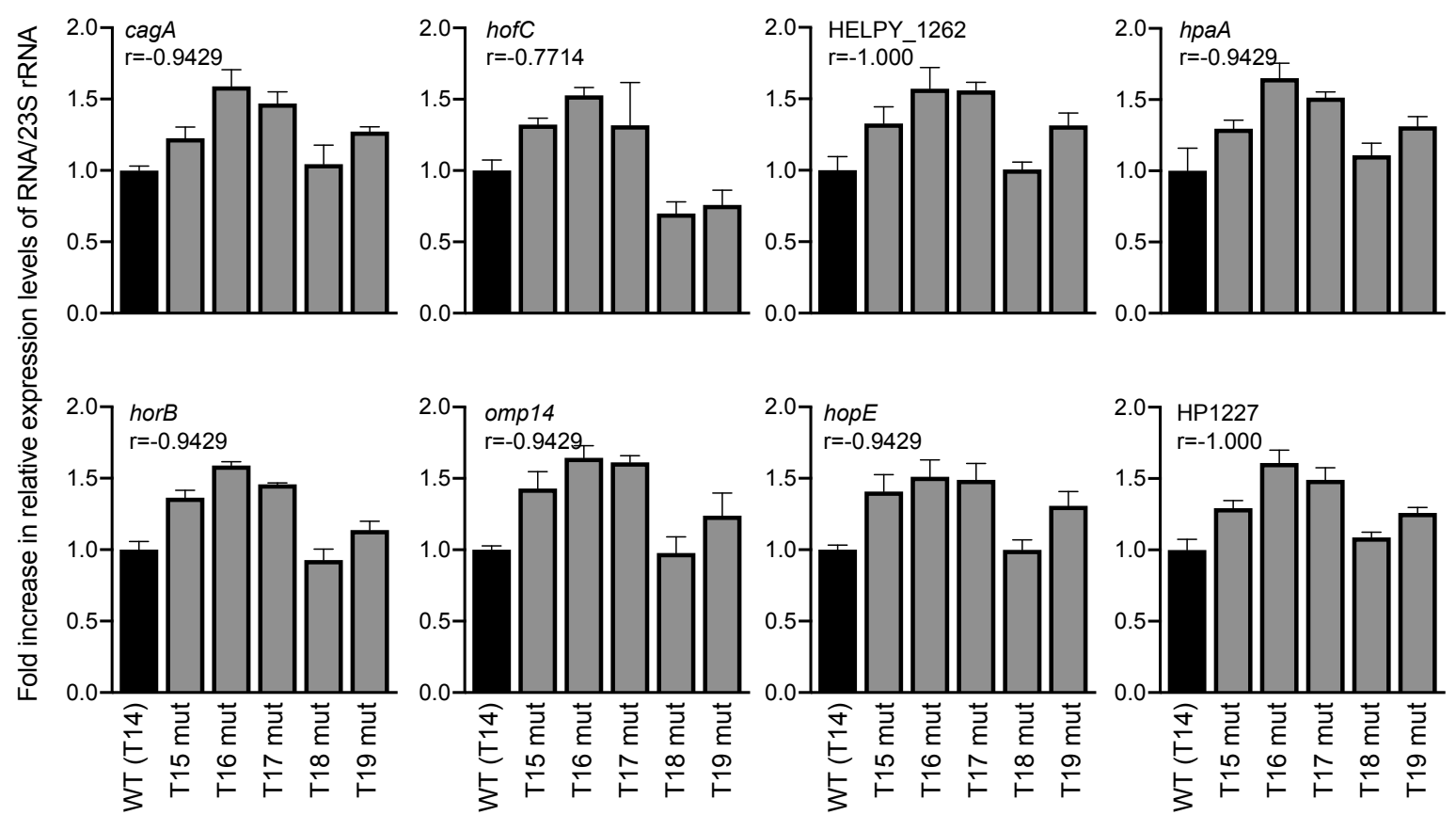


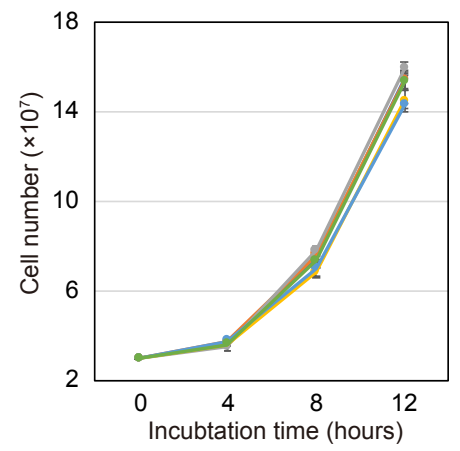

$\rightarrow$ WT

- WT / pHel2

WT / pHel2-hpnc4160

$\Delta h p n c 4160 / 4170$

- $\Delta$ hpnc4160/4170/ pHel2

- $\Delta$ hpnc4160/4170/ pHel2-hpnc4160

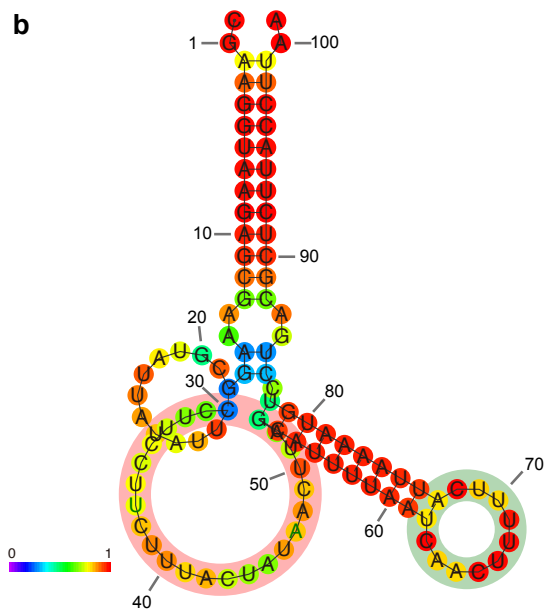

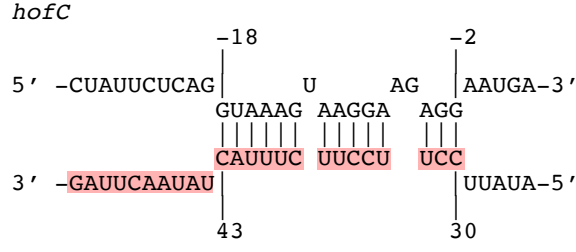

horB

5' -Aguagagaahaugu $\left.\left.\right|_{\text {Agahgahag }} ^{-15}\right|_{\text {Aauacauga-3' }} ^{-6}$

UCUUCCUUCC

3' -gauucanuaucauu $\left.\left.\right|_{39}\right|_{30} ^{\text {UCuUccuucc }}$

HP 1227

5' -AAACGA $\left.\left.\right|_{\text {UgAaAaAg G UU }} ^{2}\right|_{\text {AUCAT-3 }} ^{12}$,

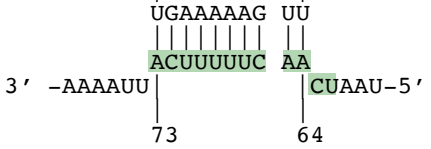
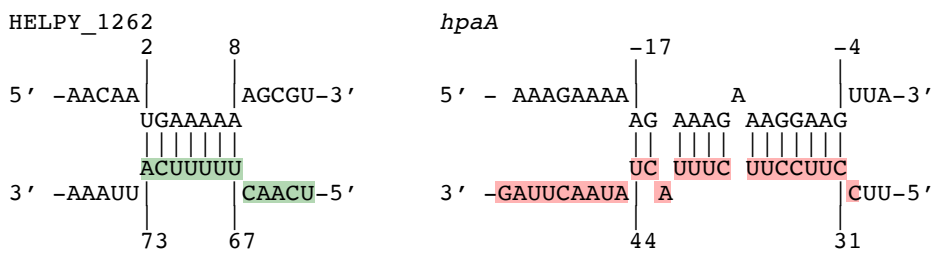

omp14

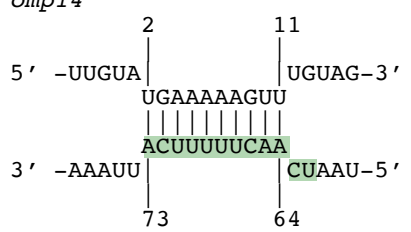

hopE

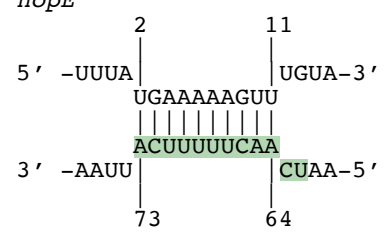

d HPnc4160 binding region CM/CRPIA motif
(amino acids sequence)

TTCCCTTTGAAAAGGCATGATAAAGTTGATGATCTCAGTAAGGTAGGG

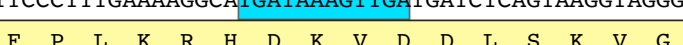

e

TYPE 1

\section{f}

5' -UAACG

TYPE 2

i $\mathrm{kDa}$

250

150

75

50

37

g

TYPE 2 NB-cagA (unbound form)

TYPE 1 NB-cagA (unbound form)

25

20
5' -UAACC C C G CAAU-3'

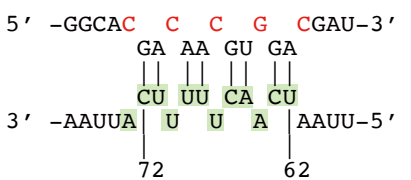

|| || || ||

CU UU CA CU

U U A AAUU-5, 


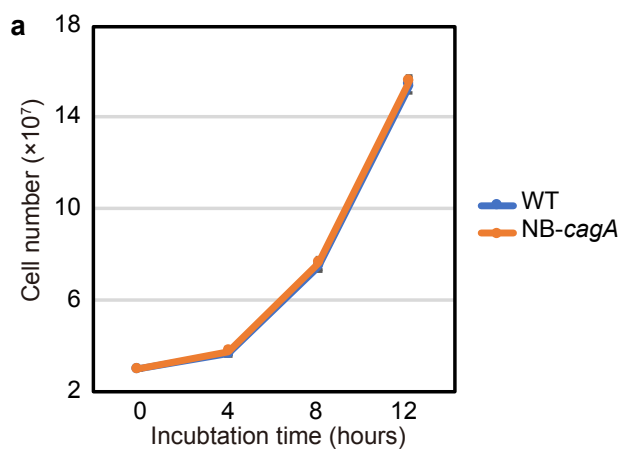

b

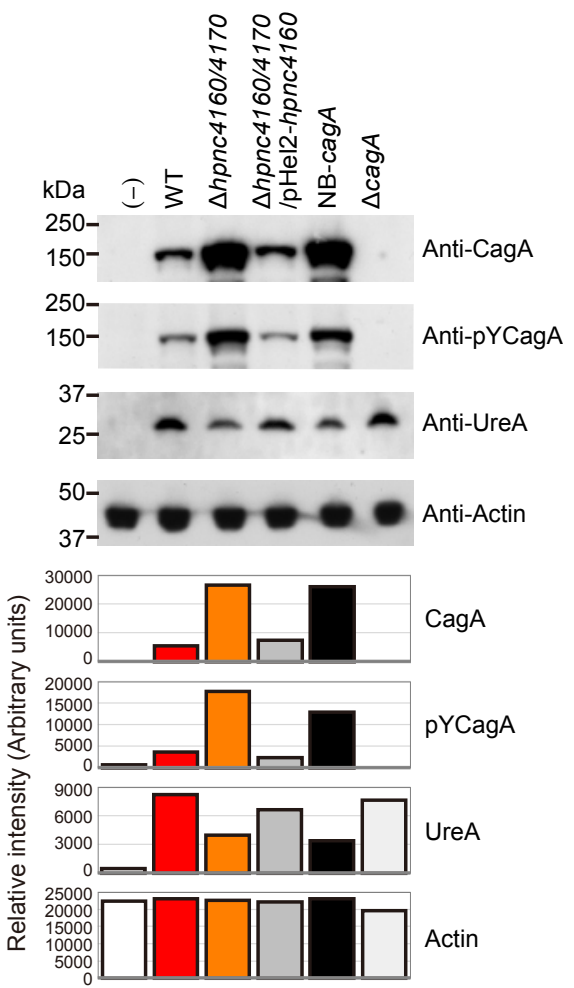

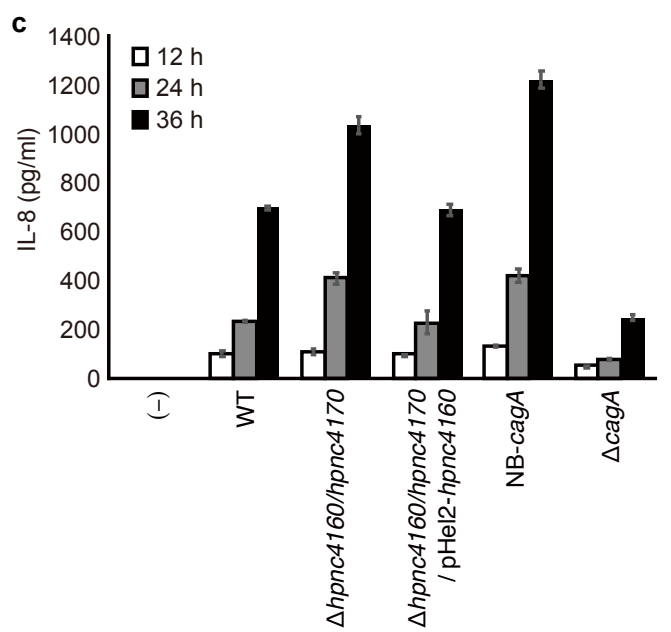


a
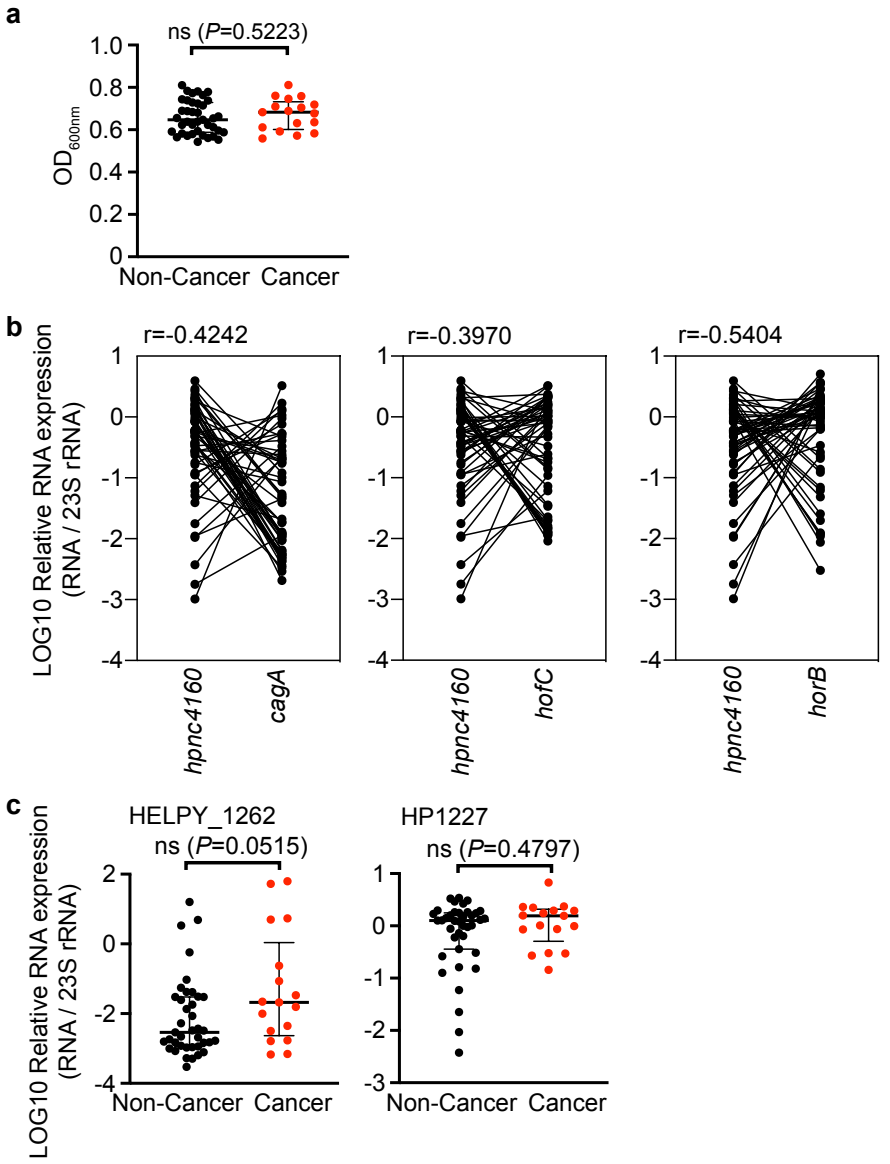

HP1227

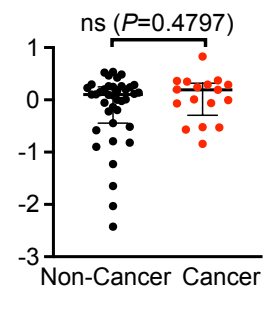


in vitro

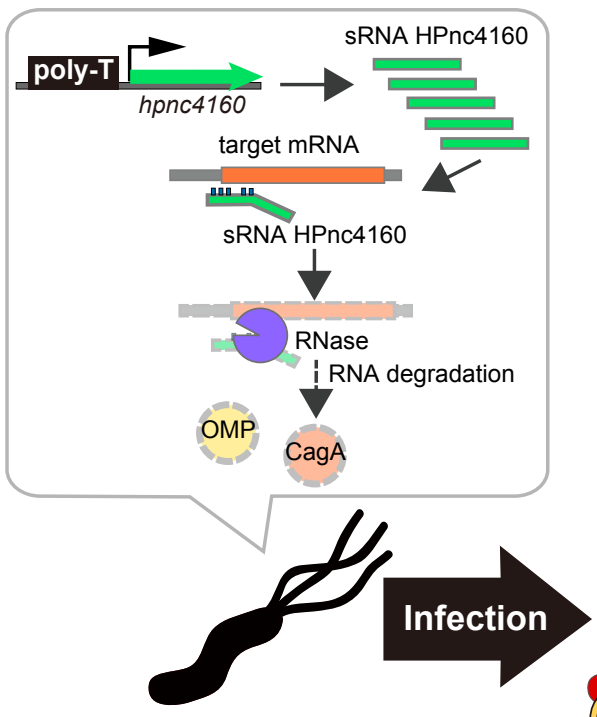

in vivo

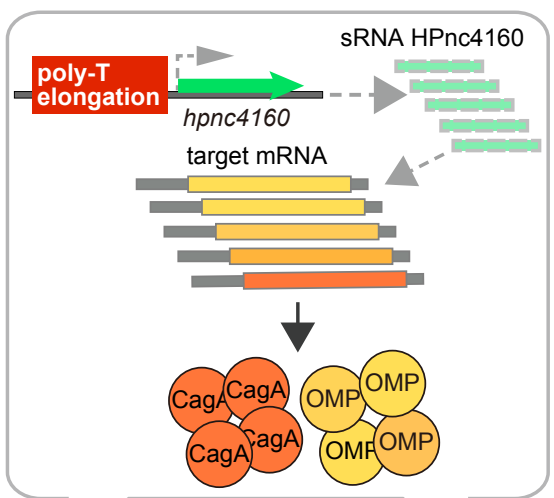

Persistent infection

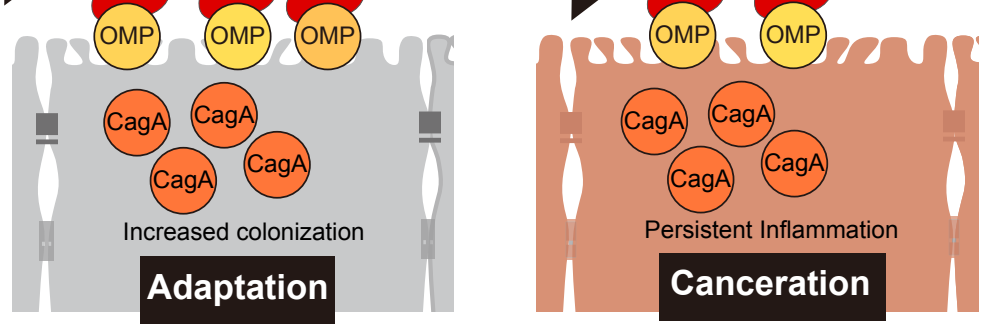




\begin{tabular}{|c|c|c|c|c|c|c|c|c|c|c|c|}
\hline \multirow{2}{*}{$\begin{array}{l}\text { Region } \\
\text { No. }\end{array}$} & \multicolumn{2}{|c|}{ No. of mutated strains (\%) } & \multicolumn{3}{|c|}{ Annotation } & \multicolumn{3}{|c|}{ Gene nearby the intergenic region (\#1) } & \multicolumn{3}{|c|}{ Gene nearby the intergenic region (\#2) } \\
\hline & $\begin{array}{c}\text { Mongolian } \\
\text { gerbil }(n=40)\end{array}$ & $\begin{array}{c}\text { C57BL/6 } \\
(n=10)\end{array}$ & $\begin{array}{l}\text { Gene } \\
\text { name }\end{array}$ & Locus tag & Gene description & $\begin{array}{l}\text { Gene } \\
\text { name }\end{array}$ & Locus tag & Gene description & Gene name & Locus tag & Gene description \\
\hline $\mathrm{R} 1$ & 52.5 & $N / A$ & $N / A$ & $\mathrm{~N} / \mathrm{A}$ & $\begin{array}{l}\text { intergenic: no } \\
\text { annotation }\end{array}$ & hopZ & HPG27_8 & $\begin{array}{l}\text { outer membrane } \\
\text { protein }\end{array}$ & & & \\
\hline $\mathrm{R} 2$ & $\mathrm{~N} / \mathrm{A}$ & 100 & futT2 & HPP12_0096 & $\begin{array}{c}\text { alpha-1,2- } \\
\text { fucosyltransferase }\end{array}$ & & & & & & \\
\hline R3 & 50 & 100 & $\mathrm{~N} / \mathrm{A}$ & $\mathrm{N} / \mathrm{A}$ & $\begin{array}{l}\text { intergenic: no } \\
\text { annotation }\end{array}$ & $\mathrm{tlpB}$ & HP0103 & $\begin{array}{l}\text { methyl-accepting } \\
\text { chemotaxis protein }\end{array}$ & & & \\
\hline $\mathrm{R} 4$ & 62.5 & $N / A$ & aras & jhp0151 & $\begin{array}{l}\text { signal-transducing } \\
\text { protein, histidine }\end{array}$ & & & & & & \\
\hline R5 & 80 & 100 & tpiA & HP0194 & $\begin{array}{l}\text { triosephosphate } \\
\text { isomerase }\end{array}$ & & & & & & \\
\hline $\mathrm{R} 6$ & N/A & 100 & HP0217 & HP0217 & hypothetical protein & & & & & & \\
\hline R7 & 72.5 & 100 & $\mathrm{~N} / \mathrm{A}$ & $\mathrm{N} / \mathrm{A}$ & $\begin{array}{l}\text { intergenic: no } \\
\text { annotation }\end{array}$ & babA & $\begin{array}{c}\text { HP1243/ } \\
\text { HPG27_298 }\end{array}$ & $\begin{array}{l}\text { outer membrane } \\
\text { protein }\end{array}$ & & & \\
\hline $\mathrm{R} 8$ & 80 & $\mathrm{~N} / \mathrm{A}$ & $\mathrm{N} / \mathrm{A}$ & $\mathrm{N} / \mathrm{A}$ & $\begin{array}{l}\text { intergenic: no } \\
\text { annotation }\end{array}$ & jhp1163 & jhp1163 & hypothetical protein & babA & $\begin{array}{l}\text { HP1243/ } \\
\text { HPG27_298* }\end{array}$ & $\begin{array}{l}\text { outer membrane } \\
\text { protein }\end{array}$ \\
\hline R9 & $\mathrm{N} / \mathrm{A}$ & 100 & $\mathrm{~N} / \mathrm{A}$ & $\mathrm{N} / \mathrm{A}$ & $\begin{array}{l}\text { intergenic: no } \\
\text { annotation }\end{array}$ & IceA2 & $\begin{array}{l}\text { HPATCC43 } \\
504 \_00587\end{array}$ & $\begin{array}{l}\text { induced by contact } \\
\text { with epithelium gene }\end{array}$ & & & \\
\hline $\mathrm{R} 10$ & 60 & $\mathrm{~N} / \mathrm{A}$ & pldA & HP0499 & phospholipase A1 & & & & & & \\
\hline R11 & 70 & 100 & $\mathrm{~N} / \mathrm{A}$ & $\mathrm{N} / \mathrm{A}$ & $\begin{array}{l}\text { intergenic: no } \\
\text { annotation }\end{array}$ & jhp0540 & jhp0540 & HAD superfamily & & & \\
\hline $\mathrm{R} 12$ & 52.5 & $\mathrm{~N} / \mathrm{A}$ & HPB8_818 & HPB8_818 & $\begin{array}{l}\text { family } 25 \text { glycosyl } \\
\text { transferase }\end{array}$ & & & & & & \\
\hline $\mathrm{R} 13$ & 62.5 & 100 & $\mathrm{~N} / \mathrm{A}$ & $\mathrm{N} / \mathrm{A}$ & $\begin{array}{l}\text { intergenic: no } \\
\text { annotation }\end{array}$ & sabA & HPG27_680 & $\begin{array}{l}\text { outer membrane } \\
\text { protein }\end{array}$ & & & \\
\hline R15 & 92.5 & 100 & $\mathrm{~N} / \mathrm{A}$ & $\mathrm{N} / \mathrm{A}$ & $\begin{array}{l}\text { intergenic: no } \\
\text { annotation }\end{array}$ & HP0947 & HP0947 & hypothetical protein & & & \\
\hline R16 & 57.5 & 100 & HP1354 & HP1354 & $\begin{array}{c}\text { adenine-specific } \\
\text { DNA }\end{array}$ & & & & & & \\
\hline R17 & $\mathrm{N} / \mathrm{A}$ & 100 & $\mathrm{~N} / \mathrm{A}$ & N/A & $\begin{array}{l}\text { intergenic: no } \\
\text { annotation }\end{array}$ & bioB & HP1406 & biotin synthase & HELPY_1371 & HELPY_1371 & $\begin{array}{l}\text { type III restriction } \\
\text { enzyme R protein }\end{array}$ \\
\hline
\end{tabular}

Extended Data Table 1 Kinoshita et al. 
a

\begin{tabular}{lrr}
\hline Gene name & $\begin{array}{l}\text { Average LOG2 } \\
\text { normalized expression }\end{array}$ & $\begin{array}{l}\text { LOG2 fold change } \\
(\Delta h p n c 4160 / 4170 / \mathrm{WT})\end{array}$ \\
\hline cagA & 15.6679564 & 0.8345757 \\
vacA3 & 13.0181033 & 1.3000122 \\
urel & 12.8122441 & 0.7256751 \\
hofC & 12.7562529 & 1.2385235 \\
HP1227 & 11.1439545 & 0.5356426 \\
hpaA & 10.9856930 & 1.3994257 \\
horB & 10.9589406 & 1.0351050 \\
omp14 & 10.8268799 & 1.3977712 \\
hopE & 10.8143652 & 1.0951572 \\
ureE & 10.6269817 & -1.1164069 \\
HELPY_1262 & 10.6234286 & 0.9346910 \\
pAL226p12 & 9.6669233 & 0.8237894 \\
HP0487 & 9.1803234 & 1.7213025 \\
HELPY_0813 & 8.5854796 & 0.8765494 \\
fliK & 7.2801813 & -0.3748484 \\
mraW & 7.0680550 & 2.0913744 \\
HPAG1_1315 & 7.0164749 & -1.5753123 \\
\hline & &
\end{tabular}

b

\begin{tabular}{lrr}
\hline Gene name & $\begin{array}{r}\text { Fold change (LOG2, } \\
\Delta \text { hpnc4160/hpnc4170 / WT) }\end{array}$ & Pval (LOG10) \\
\hline CagA & 0.6831125 & -16.0000000 \\
HofC & 1.1079556 & -14.8875441 \\
HpaA & 1.4153801 & -14.5034266 \\
GroL & 0.2561653 & -10.0187654 \\
HELPY_1262 & 0.6268596 & -9.9953361 \\
HopE & 1.1672937 & -7.7135456 \\
HP1409 & 0.1735112 & -3.6271647 \\
CeuE & 0.1737671 & -3.2477470 \\
NrdA & -0.1246917 & -2.8700087 \\
HorB & 0.8583789 & -2.6608050 \\
DnaK & 0.1192898 & -2.6579389 \\
GroES & 0.3291810 & -2.6528395 \\
Rp19 & 0.2299570 & -2.6372507 \\
HP0305 & 0.2674759 & -2.5150013 \\
HopQ & 0.1519887 & -2.4589011 \\
Omp14 & 1.1557492 & -2.4194920 \\
FlaB & -0.1694201 & -2.4003455 \\
Tuf & 0.1548424 & -2.3314305 \\
RpoB & -0.0584429 & -2.2822269 \\
Lpp20 & 0.1944653 & -2.1385174 \\
HP1227 & 0.3642362 & -2.0521036 \\
\hline & &
\end{tabular}


bioRxiv preprint doi: https://doi.org/10.1101/2020.02.15.950279; this version posted February 21, 2020. The copyright holder for this

preprint (which was not certified by peer review) is the author/funder. All rights reserved. No reuse allowed without permission.

Supplementary Information 1| Summary of mutations in the isolates recovered from $\mathrm{H}$. pylori-infected Mongolian gerbils.

Number of mutations in the isolates of 40 strains recovered from $\mathrm{H}$. pylori-infected Mongolian gerbils' stomachs 8 weeks after post infection were listed.

\begin{tabular}{|c|c|c|c|c|c|c|c|c|c|c|c|}
\hline \multirow{2}{*}{$\begin{array}{c}\text { Animal } \\
\text { No. }\end{array}$} & \multirow{2}{*}{$\begin{array}{l}\text { Strain } \\
\text { Name }\end{array}$} & \multirow{2}{*}{$\begin{array}{c}\text { Total No. } \\
\text { of } \\
\text { mutations }\end{array}$} & \multicolumn{4}{|c|}{ SNPs (single nucleotide polymorphysms) } & \multicolumn{3}{|c|}{ indel (insertion/deletion) } & \multirow{2}{*}{$\begin{array}{c}\text { SNPs rate } \\
\text { per base } \\
\text { per year }\end{array}$} & \multirow{2}{*}{$\begin{array}{l}\text { indel rate } \\
\text { per base } \\
\text { per year }\end{array}$} \\
\hline & & & total & $\begin{array}{c}\text { nonsynon } \\
\text { ymous }\end{array}$ & $\begin{array}{c}\text { synonymo } \\
\text { us }\end{array}$ & intergenic & Total & genic & intergenic & & \\
\hline \multirow{4}{*}{ \#1 } & S41 & 50 & 37 & 15 & 18 & 4 & 13 & 5 & 8 & 1.43E-04 & $5.04 \mathrm{E}-05$ \\
\hline & S42 & 48 & 40 & 15 & 20 & 5 & 8 & 2 & 6 & 1.55E-04 & $3.10 \mathrm{E}-05$ \\
\hline & $\mathrm{S} 43$ & 44 & 38 & 12 & 21 & 5 & 6 & 4 & 2 & 1.47E-04 & $2.33 \mathrm{E}-05$ \\
\hline & S44 & 46 & 41 & 16 & 21 & 4 & 5 & 1 & 4 & $1.59 \mathrm{E}-04$ & $1.94 \mathrm{E}-05$ \\
\hline \multirow{4}{*}{ \#2 } & S45 & 48 & 39 & 15 & 17 & 7 & 9 & 2 & 7 & $1.51 \mathrm{E}-04$ & $3.49 \mathrm{E}-05$ \\
\hline & $S 46$ & 47 & 38 & 22 & 14 & 2 & 9 & 1 & 8 & $1.47 \mathrm{E}-04$ & $3.49 \mathrm{E}-05$ \\
\hline & S47 & 46 & 36 & 15 & 15 & 6 & 10 & 2 & 8 & $1.40 \mathrm{E}-04$ & $3.88 \mathrm{E}-05$ \\
\hline & $\mathrm{S} 48$ & 48 & 41 & 20 & 16 & 5 & 7 & 2 & 5 & $1.59 \mathrm{E}-04$ & $2.71 \mathrm{E}-05$ \\
\hline \multirow{4}{*}{ \#3 } & S49 & 17 & 12 & 7 & 3 & 2 & 5 & 4 & 1 & 4.65E-05 & $1.94 \mathrm{E}-05$ \\
\hline & S50 & 26 & 18 & 8 & 8 & 2 & 7 & 3 & 4 & $6.98 \mathrm{E}-05$ & $2.71 \mathrm{E}-05$ \\
\hline & S51 & 17 & 11 & 7 & 2 & 2 & 6 & 3 & 3 & 4.27E-05 & 2.33E-05 \\
\hline & S52 & 20 & 13 & 7 & 2 & 4 & 7 & 4 & 3 & $5.04 \mathrm{E}-05$ & $2.71 \mathrm{E}-05$ \\
\hline \multirow{4}{*}{ \#4 } & S53 & 27 & 16 & 7 & 6 & 3 & 11 & 5 & 6 & $6.20 \mathrm{E}-05$ & 4.27E-05 \\
\hline & S54 & 15 & 10 & 4 & 5 & 1 & 5 & 1 & 4 & $3.88 \mathrm{E}-05$ & 1.94E-05 \\
\hline & S55 & 24 & 14 & 5 & 5 & 4 & 10 & 3 & 7 & 5.43E-05 & $3.88 \mathrm{E}-05$ \\
\hline & S56 & 25 & 13 & 5 & 6 & 2 & 12 & 4 & 8 & $5.04 \mathrm{E}-05$ & 4.65E-05 \\
\hline \multirow{4}{*}{$\# 5$} & S57 & 25 & 15 & 5 & 7 & 3 & 10 & 4 & 6 & 5.82E-05 & $3.88 \mathrm{E}-05$ \\
\hline & S58 & 29 & 15 & 5 & 5 & 5 & 14 & 5 & 9 & 5.82E-05 & 5.43E-05 \\
\hline & S59 & 23 & 13 & 5 & 6 & 2 & 10 & 2 & 8 & 5.04E-05 & $3.88 \mathrm{E}-05$ \\
\hline & $\mathrm{S} 60$ & 27 & 16 & 5 & 7 & 4 & 11 & 3 & 8 & $6.20 \mathrm{E}-05$ & 4.27E-05 \\
\hline \multirow{4}{*}{ \#6 } & S61 & 29 & 20 & 8 & 8 & 4 & 9 & 2 & 7 & $7.76 \mathrm{E}-05$ & $3.49 \mathrm{E}-05$ \\
\hline & $\mathrm{S} 62$ & 35 & 25 & 10 & 12 & 3 & 10 & 2 & 8 & $9.69 \mathrm{E}-05$ & $3.88 \mathrm{E}-05$ \\
\hline & S63 & 25 & 16 & 6 & 5 & 5 & 9 & 3 & 6 & $6.20 \mathrm{E}-05$ & $3.49 \mathrm{E}-05$ \\
\hline & S64 & 36 & 28 & 14 & 10 & 4 & 8 & 2 & 6 & $1.09 \mathrm{E}-04$ & $3.10 \mathrm{E}-05$ \\
\hline \multirow{4}{*}{ \#7 } & S65 & 50 & 39 & 14 & 21 & 4 & 11 & 5 & 6 & $1.51 \mathrm{E}-04$ & $4.27 \mathrm{E}-05$ \\
\hline & S66 & 47 & 39 & 13 & 22 & 4 & 8 & 5 & 3 & $1.51 \mathrm{E}-04$ & $3.10 \mathrm{E}-05$ \\
\hline & S67 & 55 & 44 & 15 & 22 & 7 & 11 & 4 & 7 & $1.71 \mathrm{E}-04$ & 4.27E-05 \\
\hline & S68 & 54 & 44 & 14 & 23 & 7 & 10 & 4 & 6 & $1.71 \mathrm{E}-04$ & $3.88 \mathrm{E}-05$ \\
\hline \multirow{4}{*}{$\# 8$} & S69 & 52 & 41 & 21 & 17 & 3 & 11 & 3 & 8 & $1.59 \mathrm{E}-04$ & $4.27 \mathrm{E}-05$ \\
\hline & $\mathrm{S} 70$ & 61 & 52 & 17 & 30 & 5 & 9 & 2 & 7 & 2.02E-04 & $3.49 \mathrm{E}-05$ \\
\hline & S71 & 41 & 33 & 15 & 14 & 4 & 7 & 1 & 6 & $1.28 \mathrm{E}-04$ & $2.71 \mathrm{E}-05$ \\
\hline & $\mathrm{S} 72$ & 39 & 34 & 15 & 16 & 3 & 5 & 0 & 5 & $1.32 \mathrm{E}-04$ & 1.94E-05 \\
\hline \multirow{4}{*}{$\# 9$} & $\mathrm{~S} 73$ & 43 & 37 & 17 & 15 & 4 & 6 & 2 & 4 & 1.43E-04 & 2.33E-05 \\
\hline & $\mathrm{S} 74$ & 79 & 70 & 32 & 31 & 7 & 9 & 2 & 7 & $2.71 \mathrm{E}-04$ & $3.49 \mathrm{E}-05$ \\
\hline & S75 & 71 & 63 & 29 & 26 & 8 & 8 & 4 & 4 & $2.44 \mathrm{E}-04$ & $3.10 \mathrm{E}-05$ \\
\hline & S76 & 43 & 34 & 15 & 17 & 2 & 9 & 3 & 6 & $1.32 \mathrm{E}-04$ & 3.49E-05 \\
\hline \multirow{6}{*}{$\# 10$} & S77 & 45 & 36 & 8 & 6 & 21 & 9 & 3 & 6 & $1.40 \mathrm{E}-04$ & $3.49 \mathrm{E}-05$ \\
\hline & S78 & 41 & 34 & 9 & 5 & 20 & 7 & 2 & 5 & $1.32 \mathrm{E}-04$ & $2.71 \mathrm{E}-05$ \\
\hline & S79 & 41 & 32 & 5 & 6 & 21 & 9 & 3 & 6 & $1.24 \mathrm{E}-04$ & $3.49 \mathrm{E}-05$ \\
\hline & $\mathrm{S} 80$ & 41 & 32 & 7 & 5 & 20 & 9 & 3 & 6 & $1.24 \mathrm{E}-04$ & $3.49 \mathrm{E}-05$ \\
\hline & & & & & & & & & Average & 1.19E-04 & 3.38E-05 \\
\hline & & & & & & & & & SD & $5.60 \mathrm{E}-05$ & $8.51 \mathrm{E}-06$ \\
\hline
\end{tabular}


bioRxiv preprint doi: https://doi.org/10.1101/2020.02.15.950279; this version posted February 21, 2020. The copyright holder for this

preprint (which was not certified by peer review) is the author/funder. All rights reserved. No reuse allowed without permission.

\begin{tabular}{|c|c|c|c|c|c|c|c|c|}
\hline $\begin{array}{l}\text { Strain } \\
\text { name }\end{array}$ & $\begin{array}{l}\text { Position in } \\
\text { ATCC43504 }\end{array}$ & ATCC43504 & Reisolate & & Type of difference & Annotation & $\begin{array}{c}\text { Gene nearby the } \\
\text { intergenic region (\#1) }\end{array}$ & $\begin{array}{c}\text { Gene nearby the } \\
\text { intergenic region (\#2) }\end{array}$ \\
\hline $\mathrm{S41}$ & 89186 & $\mathrm{~T}$ & c & SNP & intergenic & & HP0995 & \\
\hline 541 & 99327 & $\mathrm{c}$ & $\mathrm{T}$ & SNP & synonymous & jhp0935 & & \\
\hline 541 & 168379 & $\mathrm{c}$ & $\mathrm{T}$ & SNP & synonymous & HP1547 & & \\
\hline $\mathrm{s} 41$ & 199597 & c & $\mathrm{T}$ & SNP & intergenic & & HP1582 & jhp1488 \\
\hline 541 & 204483 & $\begin{array}{l}\text { CGTTCCGCTTTCAGTCTCGCTTCTTGTTCCGCTTTCA } \\
\text { GTCTCGCTTCTTGTTCCGCTTTCAGTCTCGCTTCTT } \\
\text { GTTCCGCTTTCA }\end{array}$ & CGTTCCGCTTTCA & indel & frameshift deletion & HPATCC43504_00192 & & \\
\hline 541 & 232964 & $\mathrm{c}$ & $\mathrm{A}$ & SNP & intergenic & & HPPC_00115 & \\
\hline 541 & 276989 & $\mathrm{G}$ & $\mathrm{A}$ & SNP & nonsynonymous & HPP12_0070 & & \\
\hline 541 & 315327 & AсCССССССCC & АсСССССССССC & indel & intergenic & & HP0103 & \\
\hline$\$ 41$ & 374756 & cGGGGGGGGGGGG & CGGGGGGGGGGGGGG & indel & frameshift insertion & jhp0151 & & \\
\hline 541 & 430922 & $\mathrm{G}$ & A & SNP & nonsynonymous & HP0194 & & \\
\hline$\$ 41$ & 455599 & TGGGGGGGGGG & TGGGGGGGGG & indel & frameshift deletion & HP0217 & & \\
\hline 541 & 546511 & $\begin{array}{l}\text { CAAAAAAAAAAAA } \\
\end{array}$ & CAAAAAAAAAAAAA & indel & intergenic & & HP1243/HPG27_298 & \\
\hline 541 & 672079 & $\mathrm{c}$ & $\mathrm{A}$ & SNP & nonsynonymous & HP1134 & & \\
\hline$\$ 41$ & 691071 & G & A & SNP & synonymous & HP0489 & & \\
\hline 541 & 691074 & A & c & SNP & synonymous & HP0489 & & \\
\hline$\$ 41$ & 691077 & $\mathrm{G}$ & A & SNP & nonsynonymous & HP0489 & & \\
\hline 541 & 691089 & $\mathrm{~A}$ & G & SNP & synonymous & HP0489 & & \\
\hline$S 41$ & 691091 & $\mathrm{c}$ & A & SNP & nonsynonymous & HP0489 & & \\
\hline 541 & 691101 & $\mathrm{~T}$ & c & SNP & synonymous & HP0489 & & \\
\hline 541 & 691113 & G & $\mathrm{T}$ & SNP & nonsynonymous & HP0489 & & \\
\hline 541 & 691157 & $\mathrm{c}$ & A & SNP & nonsynonymous & HP0489 & & \\
\hline$\$ 41$ & 691173 & $\mathrm{c}$ & A & SNP & synonymous & HP0489 & & \\
\hline 541 & 691175 & $\mathrm{~A}$ & G & SNP & nonsynonymous & HP0489 & & \\
\hline 541 & 691179 & $\mathrm{c}$ & $\mathrm{G}$ & SNP & synonymous & HP0489 & & \\
\hline 541 & 691185 & $\mathrm{~A}$ & $G$ & SNP & nonsynonymous & HP0489 & & \\
\hline 541 & 691189 & $\mathrm{c}$ & G & SNP & nonsynonymous & HP0489 & & \\
\hline 541 & 691190 & A & c & SNP & nonsynonymous & HP0489 & & \\
\hline 541 & 801836 & GAAAAAAAAAAAA & GAAAAAAAAAAAAA & indel & intergenic & & jhp0540 & \\
\hline 541 & 830053 & $\mathrm{c}$ & $\mathrm{T}$ & SNP & nonsynonymous & HPP12_0617 & & \\
\hline 541 & 839999 & $\mathrm{~T}$ & c & SNP & nonsynonymous & HPB8_818 & & \\
\hline 541 & 873661 & $\mathrm{c}$ & $T$ & SNP & synonymous & HP0651 & & \\
\hline 541 & 873681 & G & A & SNP & synonymous & HP0651 & & \\
\hline 541 & 873702 & G & $\mathrm{T}$ & SNP & synonymous & HP0651 & & \\
\hline 541 & 873717 & c & $\mathrm{T}$ & SNP & synonymous & HP0651 & & \\
\hline 541 & 879985 & $\mathrm{c}$ & $\mathrm{T}$ & SNP & nonsynonymous & HP0656 & & \\
\hline$S 41$ & 952086 & ТтСТстстстстстстС & TТСТСТСТСТСТСТC & indel & intergenic & & HPG27_677 & \\
\hline 541 & 958574 & CGAGAGAGAGAGAG & CGAGAGAGAGAGAGAG & indel & intergenic & . & HPG27_680 & \\
\hline 541 & 958719 & $\mathrm{G}$ & A & SNP & intergenic & 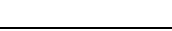 & HPG27_680 & \\
\hline$\$ 41$ & 1043704 & GTTTTTTTTTTTTT & GTTTTTTTTTTTTTTTT & indel & frameshift insertion/intergenic & HPnc4170 & HP0811 & HPnc4160 \\
\hline 541 & 1187376 & АТTTTTTT & ATTTTTTTT & indel & intergenic & & HP0947 & \\
\hline$\$ 41$ & 1332690 & $\mathrm{c}$ & A & SNP & synonymous & HPATCC43504_01275 & & \\
\hline 541 & 1332954 & $\mathrm{~T}$ & G & SNP & synonymous & HPATCC43504_01275 & & \\
\hline 541 & 1332987 & $\mathrm{~T}$ & $\mathrm{c}$ & SNP & synonymous & HPATCC43504_01275 & & \\
\hline 541 & 1332996 & $\mathrm{~T}$ & $\mathrm{G}$ & SNP & synonymous & HPATCC43504_01275 & & \\
\hline$\$ 41$ & 1332997 & G & c & SNP & nonsynonymous & HPATCC43504_01275 & & \\
\hline 541 & 1332999 & $\mathrm{~T}$ & c & SNP & synonymous & HPATCC43504_01275 & & \\
\hline 541 & 1582085 & TTT & ТTTTTT & indel & intergenic & & HELPY_1317 & HP0228 \\
\hline 541 & 1592784 & ATGTG & ATG & indel & frameshift deletion & HP1354 & & \\
\hline 541 & 1592801 & $\mathrm{~T}$ & G & SNP & synonymous & HP1354 & & \\
\hline 541 & 1593602 & TGGGGGGGGGGG & TGGGGGGGGGGGG & indel & frameshift insertion & HP1354 & & \\
\hline 542 & 99327 & $\mathrm{c}$ & $T$ & SNP & synonymous & jhp0935 & & \\
\hline 542 & 168379 & c & $\mathrm{T}$ & SNP & synonymous & HP1547 & & \\
\hline 542 & 199597 & $\mathrm{c}$ & $\mathrm{T}$ & SNP & intergenic & & HP1582 & jhp1488 \\
\hline 542 & 276989 & G & $\mathrm{A}$ & SNP & nonsynonymous & HPP12_0070 & & \\
\hline 542 & 372979 & $\mathrm{~T}$ & $\mathrm{c}$ & SNP & intergenic & & HP0162 & \\
\hline 542 & 374769 & $T$ & G & SNP & nonsynonymous & jhp0151 & & \\
\hline 542 & 430922 & G & A & SNP & nonsynonymous & HP0194 & & \\
\hline 542 & 548957 & c & $\mathrm{T}$ & SNP & intergenic & & jhp1163 & HPG27_298 \\
\hline$\$ 42$ & 548965 & c & $T$ & SNP & intergenic & & jhp1163 & HPG27_298 \\
\hline 542 & 548978 & G & $\mathrm{T}$ & SNP & intergenic & & jhp1163 & HPG27_298 \\
\hline 542 & 672079 & $\mathrm{c}$ & A & SNP & nonsynonymous & HP1134 & & \\
\hline 542 & 691071 & G & A & SNP & synonymous & HP0489 & & \\
\hline 542 & 691074 & A & c & SNP & synonymous & HP0489 & & \\
\hline 542 & 691077 & G & A & SNP & nonsynonymous & HP0489 & & \\
\hline 542 & 691089 & A & G & SNP & synonymous & HP0489 & & \\
\hline$\$ 42$ & 691091 & $\mathrm{C}$ & $\mathrm{A}$ & SNP & nonsynonymous & HP0489 & & \\
\hline 542 & 691101 & $\mathrm{~T}$ & c & SNP & synonymous & HP0489 & & \\
\hline 542 & 691113 & G & $\mathrm{T}$ & SNP & nonsynonymous & HP0489 & & \\
\hline 542 & 691157 & c & $\mathrm{A}$ & SNP & nonsynonymous & HP0489 & & \\
\hline 542 & 691173 & c & A & SNP & synonymous & HP0489 & & \\
\hline 542 & 691175 & A & G & SNP & nonsynonymous & HP0489 & & \\
\hline 542 & 691179 & c & G & SNP & synonymous & HP0489 & & \\
\hline 542 & 691185 & A & $G$ & SNP & nonsynonymous & HP0489 & & \\
\hline 542 & 691189 & c & G & SNP & nonsynonymous & HP0489 & & \\
\hline 542 & 691190 & A & c & SNP & nonsynonymous & HP0489 & & \\
\hline$\$ 42$ & 801836 & GAAAAAAAAAAAA & GAAAAAAAAAAAAA & indel & intergenic & & jhp0540 & \\
\hline 542 & 830053 & c & $T$ & SNP & nonsynonymous & HPP12_0617 & & \\
\hline 542 & 873661 & $\mathrm{c}$ & $\mathrm{T}$ & SNP & synonymous & HP0651 & & \\
\hline 542 & 873681 & G & $\mathrm{A}$ & SNP & synonymous & HP0651 & & \\
\hline 542 & 873702 & G & $\mathrm{T}$ & SNP & synonymous & HP0651 & & \\
\hline 542 & 873717 & c & $T$ & SNP & synonymous & HP0651 & & \\
\hline 542 & 873807 & $\mathrm{c}$ & G & SNP & synonymous & HP0651 & & \\
\hline 542 & 874024 & AGGGGGGGGG & AGGGGGGGGGG & indel & intergenic & & HP0651 & \\
\hline$\$ 42$ & 879985 & $\mathrm{c}$ & $\mathrm{T}$ & SNP & nonsynonymous & HP0656 & & \\
\hline
\end{tabular}




\begin{tabular}{|c|c|c|c|c|c|c|c|c|}
\hline$\$ 42$ & 958574 & CGAGAGAGAGAGAG & CGAGAGAGAGAGAGAG & indel & intergenic & & HPG27_680 & \\
\hline 542 & 1043704 & GTTTTTTTTTTTTTT & GтTTTTTTTTTTTTTTTT & indel & frameshift insertion/intergenic & HPnc4170 & HP0811 & HPnc4160 \\
\hline$\$ 42$ & 1124346 & CGAGAGAGAGAGAGAGAGAG & CGAGAGAGAGAGAGAGAGAGAG & indel & intergenic & & HPG27_1187 & \\
\hline$\$ 42$ & 1187376 & АTTTTTTT & ATTTTTTTT & indel & intergenic & & HP0947 & \\
\hline S42 & 1332690 & c & A & SNP & synonymous & HPATCC43504_01275 & & \\
\hline 542 & 1332900 & G & $T$ & SNP & synonymous & HPATCC43504_01275 & & \\
\hline 542 & 1332906 & c & $\mathrm{T}$ & SNP & synonymous & HPATCC43504_01275 & & \\
\hline 542 & 1332954 & $\mathrm{~T}$ & G & SNP & synonymous & HPATCC43504_01275 & & \\
\hline 542 & 1332987 & $\mathrm{~T}$ & c & SNP & synonymous & HPATCC43504_01275 & & \\
\hline$\$ 42$ & 1332996 & $T$ & $G$ & SNP & synonymous & HPATCC43504_01275 & & \\
\hline 542 & 1332997 & G & c & SNP & nonsynonymous & HPATCC43504_01275 & & \\
\hline$\$ 42$ & 1332999 & $T$ & c & SNP & synonymous & HPATCC43504_01275 & & \\
\hline 542 & 1592786 & GT & G & indel & frameshift deletion & HP1354 & & \\
\hline 542 & 1663529 & GTTTTT & GTTTT & indel & 1 bp deletion & & & \\
\hline 543 & 99327 & $\mathrm{c}$ & $\mathrm{T}$ & SNP & synonymous & jhp0935 & & \\
\hline 543 & 168379 & c & $\mathrm{T}$ & SNP & synonymous & HP1547 & & \\
\hline$\$ 43$ & 199597 & c & $T$ & SNP & intergenic & & HP1582 & jhp1488 \\
\hline$\$ 43$ & 276989 & G & A & SNP & nonsynonymous & HPP12_0070 & & \\
\hline 543 & 374756 & CGGGGGGGGGGGG & CGGGGGGGGGGGGGG & indel & frameshift insertion & jhp0151 & & \\
\hline 543 & 430922 & G & A & SNP & nonsynonymous & HP0194 & & \\
\hline 543 & 466419 & TGAGAGAGAGAGAGAGAG & TGAGAGAGAGAGAGAG & indel & intergenic & & HP0227 & \\
\hline$\$ 43$ & 540566 & $\begin{array}{l}\text { GGAGATTAAACAAGAGATTAAACAAGAGATTAA } \\
\text { ACAAGAG }\end{array}$ & $\begin{array}{l}\text { GGAGATTAAACAAGAGATTAAACAAGAGATTAA } \\
\text { ACAAGAGATTAAACAAGAG }\end{array}$ & indel & frameshift insertion & & & \\
\hline 543 & 548965 & c & $T$ & SNP & intergenic & & jhp1163 & HPG27_298 \\
\hline 543 & 548978 & G & $\mathrm{T}$ & SNP & intergenic & & jhp1163 & HPG27_298 \\
\hline$\$ 43$ & 548994 & A & $T$ & SNP & intergenic & & jhp1163 & HPG27_298 \\
\hline 543 & 672079 & c & A & SNP & nonsynonymous & HP1134 & & \\
\hline 543 & 691071 & G & A & SNP & synonymous & HP0489 & & \\
\hline 543 & 691074 & A & c & SNP & synonymous & HP0489 & & \\
\hline 543 & 691077 & G & A & SNP & nonsynonymous & HP0489 & & \\
\hline 543 & 691089 & A & G & SNP & synonymous & HP0489 & & \\
\hline 543 & 691091 & c & A & SNP & nonsynonymous & HP0489 & & \\
\hline 543 & 691101 & $T$ & c & SNP & synonymous & HP0489 & & \\
\hline$\$ 43$ & 691113 & G & $T$ & SNP & nonsynonymous & HP0489 & & \\
\hline$\$ 43$ & 691173 & c & A & SNP & synonymous & HP0489 & & \\
\hline 543 & 691175 & A & G & SNP & nonsynonymous & HP0489 & & \\
\hline 543 & 691179 & c & G & SNP & synonymous & HP0489 & & \\
\hline 543 & 691185 & A & G & SNP & nonsynonymous & HP0489 & & \\
\hline$\$ 43$ & 691189 & c & G & SNP & nonsynonymous & HP0489 & & \\
\hline 543 & 691190 & A & c & SNP & nonsynonymous & HP0489 & & \\
\hline 543 & 830053 & $\mathrm{c}$ & $T$ & SNP & nonsynonymous & HPP12_0617 & & \\
\hline 543 & 873661 & c & $\mathrm{T}$ & SNP & synonymous & HP0651 & & \\
\hline 543 & 873681 & G & A & SNP & synonymous & HP0651 & & \\
\hline 543 & 873702 & G & $\mathrm{T}$ & SNP & synonymous & HP0651 & & \\
\hline 543 & 873717 & $\mathrm{c}$ & $\mathrm{T}$ & SNP & synonymous & HP0651 & & \\
\hline$\$ 43$ & 948328 & c & A & SNP & synonymous & HPP12_0726 & & \\
\hline$\$ 43$ & 1187376 & АТTTTTTT & ATTTTTTTT & indel & intergenic & & HP0947 & \\
\hline 543 & 1318527 & $\mathrm{c}$ & A & SNP & intergenic & jhp1031 & & \\
\hline 543 & 1332690 & c & A & SNP & synonymous & HPATCC43504_01275 & & \\
\hline 543 & 1332900 & G & $\mathrm{T}$ & SNP & synonymous & HPATCC43504_01275 & & \\
\hline$\$ 43$ & 1332906 & c & $T$ & SNP & synonymous & HPATCC43504_01275 & & \\
\hline 543 & 1332954 & $T$ & G & SNP & synonymous & HPATCC43504_01275 & & \\
\hline 543 & 1332987 & $\mathrm{~T}$ & c & SNP & synonymous & HPATCC43504_01275 & & \\
\hline$\$ 43$ & 1332996 & $T$ & G & SNP & synonymous & HPATCC43504_01275 & & \\
\hline$\$ 43$ & 1332997 & G & c & SNP & nonsynonymous & HPATCC43504_01275 & & \\
\hline$\$ 43$ & 1332999 & $T$ & c & SNP & synonymous & HPATCC43504_01275 & & \\
\hline 543 & 1592784 & ATGTG & ATG & indel & frameshift deletion & HP1354 & & \\
\hline 543 & 1592801 & $T$ & G & SNP & synonymous & HP1354 & & \\
\hline 543 & 1593602 & TGGGGGGGGGGG & G & indel & frameshift deletion & HP1354 & & \\
\hline 544 & 99327 & c & $T$ & SNP & synonymous & jhp0935 & & \\
\hline 544 & 168379 & c & $T$ & SNP & synonymous & HP1547 & & \\
\hline 544 & 199597 & c & $\mathrm{T}$ & SNP & intergenic & & HP1582 & jhp1488 \\
\hline 544 & 232964 & c & A & SNP & intergenic & & HPPC_00115 & \\
\hline$\$ 44$ & 276989 & G & A & SNP & nonsynonymous & HPP12_0070 & & \\
\hline 544 & 430922 & G & A & SNP & nonsynonymous & HP0194 & & \\
\hline 544 & 549326 & G & $\mathrm{T}$ & SNP & intergenic & & jhp1163 & HPG27_298 \\
\hline$\$ 44$ & 672079 & c & A & SNP & nonsynonymous & HP1134 & & \\
\hline 544 & 691071 & $G$ & A & SNP & synonymous & HP0489 & & \\
\hline 544 & 691074 & A & C & SNP & synonymous & HP0489 & & \\
\hline 544 & 691077 & G & A & SNP & nonsynonymous & HP0489 & & \\
\hline$\$ 44$ & 691089 & A & G & SNP & synonymous & HP0489 & & \\
\hline 544 & 691091 & $\mathrm{c}$ & A & SNP & nonsynonymous & HP0489 & & \\
\hline$\$ 44$ & 691101 & $T$ & c & SNP & synonymous & HP0489 & & \\
\hline$\$ 44$ & 691113 & $G$ & $T$ & SNP & nonsynonymous & HP0489 & & \\
\hline S44 & 691173 & c & A & SNP & synonymous & HP0489 & & \\
\hline 544 & 691175 & A & G & SNP & nonsynonymous & HP0489 & & \\
\hline 544 & 691179 & c & G & SNP & synonymous & HP0489 & & \\
\hline 544 & 691185 & A & G & SNP & nonsynonymous & HP0489 & & \\
\hline 544 & 691189 & $\mathrm{c}$ & G & SNP & nonsynonymous & HP0489 & & \\
\hline$\$ 44$ & 691190 & A & c & SNP & nonsynonymous & HP0489 & & \\
\hline S44 & 801836 & GAAAAAAAAAAAA & GAAAAAAAAAAAAA & indel & intergenic & & jhp0540 & \\
\hline S44 & 830053 & C & $T$ & SNP & nonsynonymous & HPP12_0617 & & \\
\hline 544 & 839999 & $T$ & c & SNP & nonsynonymous & HPB8_818 & & \\
\hline$\$ 44$ & 873661 & C & $T$ & SNP & synonymous & HP0651 & & \\
\hline 544 & 873681 & G & A & SNP & synonymous & HP0651 & & \\
\hline 544 & 873702 & $G$ & $T$ & SNP & synonymous & HP0651 & & \\
\hline$\$ 44$ & 873717 & C & $\mathrm{T}$ & SNP & synonymous & HP0651 & & \\
\hline
\end{tabular}




\begin{tabular}{|c|c|c|c|c|c|c|c|c|}
\hline$\$ 44$ & 879985 & c & $T$ & SNP & nonsynonymous & HP0656 & & \\
\hline$\$ 44$ & 958574 & CGAGAGAGAGAGAG & CGAGAGAGAGAGAGAG & indel & intergenic & & HPG27_680 & \\
\hline 544 & 1043704 & GTTTTTTTTTTTTTT & GтTTTTTTTTTTTTTTT & indel & frameshift insertion/intergenic & HPnc4170 & HP0811 & HPnc4160 \\
\hline 544 & 1066590 & $\mathrm{~T}$ & A & SNP & intergenic & & jhp0775 & \\
\hline S44 & 1187376 & ATTTTTTT & ATTTTTTTT & indel & intergenic & & HP0947 & \\
\hline 544 & 1192843 & $T$ & G & SNP & nonsynonymous & HPP12_0950 & & \\
\hline$\$ 44$ & 1252178 & c & $T$ & SNP & nonsynonymous & HP0407 & & \\
\hline$\$ 44$ & 1284281 & $T$ & c & SNP & synonymous & HPG27_1018 & & \\
\hline 544 & 1332690 & c & A & SNP & synonymous & HPATCC43504_01275 & & \\
\hline 544 & 1332900 & G & $\mathrm{T}$ & SNP & synonymous & HPATCC43504_01275 & & \\
\hline S44 & 1332906 & c & $T$ & SNP & synonymous & HPATCC43504_01275 & & \\
\hline$\$ 44$ & 1332954 & $T$ & $G$ & SNP & synonymous & HPATCC43504_01275 & & \\
\hline 544 & 1332987 & $T$ & c & SNP & synonymous & HPATCC43504_01275 & & \\
\hline$\$ 44$ & 1332996 & $T$ & G & SNP & synonymous & HPATCC43504_01275 & & \\
\hline 544 & 1332997 & $G$ & $\mathrm{c}$ & SNP & nonsynonymous & HPATCC43504_01275 & & \\
\hline 544 & 1332999 & $\mathrm{~T}$ & c & SNP & synonymous & HPATCC43504_01275 & & \\
\hline 544 & 1592784 & ATGTG & ATG & indel & frameshift deletion & HP1354 & & \\
\hline$\$ 44$ & 1592801 & $T$ & G & SNP & synonymous & HP1354 & & \\
\hline$\$ 45$ & 99327 & c & $\mathrm{T}$ & SNP & synonymous & jhp0935 & & \\
\hline$\$ 45$ & 168379 & c & $T$ & SNP & synonymous & HP1547 & & \\
\hline$\$ 45$ & 199597 & c & $T$ & SNP & intergenic & & HP1582 & jhp1488 \\
\hline 545 & 251374 & G & A & SNP & nonsynonymous & HP0045 & & \\
\hline 545 & 276989 & G & A & SNP & nonsynonymous & HPP12_0070 & & \\
\hline$\$ 45$ & 374756 & CGGGGGGGGGGGG & CGGGGGGGGGGGGGG & indel & frameshift insertion & jhp0151 & & \\
\hline$\$ 45$ & 424517 & CGGGG & CGGGGG & indel & intergenic & & HP0189 & HP0188 \\
\hline$\$ 45$ & 430922 & G & A & SNP & nonsynonymous & HP0194 & & \\
\hline$\$ 45$ & 546511 & CAAAAAAAAAAAA & CAAAAAAAAAAAAA & indel & intergenic & & HP1243/HPG27_298 & \\
\hline$\$ 45$ & 548957 & c & $T$ & SNP & intergenic & & jhp1163 & HPG27_298 \\
\hline 545 & 548965 & $\mathrm{c}$ & $\mathrm{T}$ & SNP & intergenic & & jhp1163 & HPG27_298 \\
\hline 545 & 548994 & A & $\mathrm{T}$ & SNP & intergenic & & jhp1163 & HPG27_298 \\
\hline 545 & 691071 & G & A & SNP & synonymous & HP0489 & & \\
\hline$\$ 45$ & 691074 & A & $\mathrm{c}$ & SNP & synonymous & HP0489 & & \\
\hline$\$ 45$ & 691077 & G & A & SNP & nonsynonymous & HP0489 & & \\
\hline 545 & 691089 & A & $G$ & SNP & synonymous & HP0489 & & \\
\hline$\$ 45$ & 691091 & c & A & SNP & nonsynonymous & HP0489 & & \\
\hline$\$ 45$ & 691101 & $T$ & c & SNP & synonymous & HP0489 & & \\
\hline 545 & 691113 & G & $\mathrm{T}$ & SNP & nonsynonymous & HP0489 & & \\
\hline 545 & 691157 & c & A & SNP & nonsynonymous & HP0489 & & \\
\hline$\$ 45$ & 691173 & $\mathrm{c}$ & A & SNP & synonymous & HP0489 & & \\
\hline$\$ 45$ & 691175 & A & $G$ & SNP & nonsynonymous & HP0489 & & \\
\hline$\$ 45$ & 691179 & c & $G$ & SNP & synonymous & HP0489 & & \\
\hline$\$ 45$ & 691185 & A & $G$ & SNP & nonsynonymous & HP0489 & & \\
\hline 545 & 691189 & c & G & SNP & nonsynonymous & HP0489 & & \\
\hline$\$ 45$ & 691190 & A & c & SNP & nonsynonymous & HP0489 & & \\
\hline$\$ 45$ & 748650 & $T$ & A & SNP & intergenic & & HP0514 & \\
\hline$\$ 45$ & 805851 & A & $T$ & SNP & intergenic & & HPG27_556 & \\
\hline$\$ 45$ & 830053 & c & $T$ & SNP & nonsynonymous & HPP12_0617 & & \\
\hline$\$ 45$ & 958702 & CAAAAAAAAAAAAAAAA & CAAAAAAAAAAAAAAAAAA & indel & intergenic & & HPG27_680 & \\
\hline 545 & 1043704 & GTTTTTTTTTTTTTT & GтTTTTTTTTTTTTTTT & indel & frameshift insertion/intergenic & HPnc4170 & HP0811 & HPnc4160 \\
\hline$\$ 45$ & 1187376 & АТTTTTTT & ATTTTTTTT & indel & intergenic & & HP0947 & \\
\hline 545 & 1262251 & c & A & SNP & nonsynonymous & jhp0981 & & \\
\hline$\$ 45$ & 1295885 & GATATATATATATATATAT & $\begin{array}{l}\text { GATATATATATATATATATATAT,GATATATATATA } \\
\text { TATATATAT }\end{array}$ & indel & intergenic & & HPATCC43504_01238 & \\
\hline$\$ 45$ & 1318527 & c & A & SNP & intergenic & jhp1031 & & \\
\hline$\$ 45$ & 1332690 & c & A & SNP & synonymous & HPATCC43504_01275 & & \\
\hline$\$ 45$ & 1332888 & $T$ & $G$ & SNP & synonymous & HPATCC43504_01275 & & \\
\hline 545 & 1332893 & $T$ & $G$ & SNP & nonsynonymous & HPATCC43504_01275 & & \\
\hline 545 & 1332897 & A & G & SNP & synonymous & HPATCC43504_01275 & & \\
\hline$\$ 45$ & 1332900 & G & $T$ & SNP & synonymous & HPATCC43504_01275 & & \\
\hline$\$ 45$ & 1332906 & c & $\mathrm{T}$ & SNP & synonymous & HPATCC43504_01275 & & \\
\hline$\$ 45$ & 1332954 & $T$ & $G$ & SNP & synonymous & HPATCC43504_01275 & & \\
\hline 545 & 1332987 & $T$ & $\mathrm{c}$ & SNP & synonymous & HPATCC43504_01275 & & \\
\hline$\$ 45$ & 1332996 & $T$ & G & SNP & synonymous & HPATCC43504_01275 & & \\
\hline$\$ 45$ & 1332997 & G & c & SNP & nonsynonymous & HPATCC43504_01275 & & \\
\hline$\$ 45$ & 1332999 & $T$ & c & SNP & synonymous & HPATCC43504_01275 & & \\
\hline$\$ 45$ & 1582072 & ATT & ATTTTT & indel & intergenic & & HELPY_1317 & HP0228 \\
\hline$\$ 45$ & 1592786 & GT & G & indel & frameshift deletion & HP1354 & & \\
\hline$\$ 46$ & 99327 & c & $T$ & SNP & synonymous & jhp0935 & & \\
\hline$\$ 46$ & 168379 & $\mathrm{c}$ & $T$ & SNP & synonymous & HP1547 & & \\
\hline$\$ 46$ & 199597 & c & $\mathrm{T}$ & SNP & intergenic & & HP1582 & jhp1488 \\
\hline$\$ 46$ & 251374 & G & A & SNP & nonsynonymous & HP0045 & & \\
\hline$\$ 46$ & 276989 & G & A & SNP & nonsynonymous & HPP12_0070 & & \\
\hline 546 & 315327 & ACCCCCCCCCC & ACCCCCCCCCCC & indel & intergenic & & HP0103 & \\
\hline$\$ 46$ & 374756 & CGGGGGGGGGGGG & CGGGGGGGGGGGGGG & indel & frameshift insertion & jhp0151 & & \\
\hline 546 & 424517 & CGGGG & CGGGGG & indel & intergenic & & HP0189 & HP0188 \\
\hline 546 & 430922 & G & A & SNP & nonsynonymous & HP0194 & & \\
\hline 546 & 548965 & c & $\mathrm{T}$ & SNP & intergenic & & jhp1163 & HPG27_298 \\
\hline$\$ 46$ & 691071 & G & A & SNP & synonymous & HP0489 & & \\
\hline 546 & 691074 & A & c & SNP & synonymous & HP0489 & & \\
\hline$\$ 46$ & 691077 & $G$ & A & SNP & nonsynonymous & HP0489 & & \\
\hline$\$ 46$ & 691089 & A & $G$ & SNP & synonymous & HP0489 & & \\
\hline$\$ 46$ & 691091 & c & A & SNP & nonsynonymous & HP0489 & & \\
\hline$\$ 46$ & 691101 & $T$ & $\mathrm{c}$ & SNP & synonymous & HP0489 & & \\
\hline$\$ 46$ & 691113 & G & $T$ & SNP & nonsynonymous & HP0489 & & \\
\hline S46 & 691157 & c & A & SNP & nonsynonymous & HP0489 & & \\
\hline 546 & 691173 & C & A & SNP & synonymous & HP0489 & & \\
\hline$\$ 46$ & 691175 & A & $G$ & SNP & nonsynonymous & HP0489 & & \\
\hline
\end{tabular}




\begin{tabular}{|c|c|c|c|c|c|c|c|c|}
\hline$\$ 46$ & 691179 & c & G & SNP & synonymous & HP0489 & & \\
\hline$\$ 46$ & 691185 & A & G & SNP & nonsynonymous & HP0489 & & \\
\hline 546 & 691189 & $\mathrm{c}$ & $G$ & SNP & nonsynonymous & HP0489 & & \\
\hline 546 & 691190 & A & c & SNP & nonsynonymous & HP0489 & & \\
\hline 546 & 801836 & GAAAAAAAAAAAA & GAAAAAAAAAAAAAA & indel & intergenic & & jhp0540 & \\
\hline 546 & 830053 & c & $\mathrm{T}$ & SNP & nonsynonymous & HPP12_0617 & & \\
\hline 546 & 839999 & $\mathrm{~T}$ & c & SNP & nonsynonymous & HPB8_818 & & \\
\hline$\$ 46$ & 958702 & CAAAAAAAAAAAAAAAA & CAAAAAAAAAAAAAAAAAA & indel & intergenic & & HPG27_680 & \\
\hline 546 & 1043704 & GTTTTTTTTTTTTTT & GTTTTTTTTTTTTTTTTT & indel & frameshift insertion/intergenic & HPnc4170 & HP0811 & HPnc4160 \\
\hline 546 & 1187376 & АТTTTTTT & АТTTTTTTT & indel & intergenic & & HP0947 & \\
\hline 546 & 1262251 & c & A & SNP & nonsynonymous & jhp0981 & & \\
\hline 546 & 1295885 & GATATATATATATATATAT & GATATATATATATATATATATAT & indel & intergenic & & HPATCC43504_01238 & \\
\hline 546 & 1319852 & A & AAG & indel & intergenic & & HELPY_1075 & jhp1032 \\
\hline$\$ 46$ & 1332690 & c & A & SNP & synonymous & HPATCC43504_01275 & & \\
\hline 546 & 1332954 & $T$ & $G$ & SNP & synonymous & HPATCC43504_01275 & & \\
\hline 546 & 1332987 & $T$ & c & SNP & synonymous & HPATCC43504_01275 & & \\
\hline 546 & 1332996 & $\mathrm{~T}$ & G & SNP & synonymous & HPATCC43504_01275 & & \\
\hline 546 & 1332997 & G & c & SNP & nonsynonymous & HPATCC43504_01275 & & \\
\hline 546 & 1332999 & $\mathrm{~T}$ & c & SNP & synonymous & HPATCC43504_01275 & & \\
\hline$\$ 46$ & 1482848 & G & $T$ & SNP & nonsynonymous & HPG27_298 & & \\
\hline 546 & 1482850 & $T$ & $G$ & SNP & nonsynonymous & HPG27_298 & & \\
\hline$S 46$ & 1482859 & $T$ & c & SNP & synonymous & HPG27_298 & & \\
\hline 546 & 1482860 & $\mathrm{c}$ & A & SNP & nonsynonymous & HPG27_298 & & \\
\hline S46 & 1482861 & G & A & SNP & nonsynonymous & HPG27_298 & & \\
\hline s46 & 1483092 & G & A & SNP & nonsynonymous & HPG27_298 & & \\
\hline$\$ 46$ & 1483202 & G & A & SNP & nonsynonymous & HPG27_298 & & \\
\hline 546 & 1483269 & A & $G$ & $\begin{array}{l}\text { SNP } \\
\end{array}$ & nonsynonymous & HPG27_298 & & \\
\hline 547 & 99327 & c & $\mathrm{T}$ & SNP & synonymous & jhp0935 & & \\
\hline 547 & 168379 & c & $\mathrm{T}$ & SNP & synonymous & HP1547 & & \\
\hline 547 & 199597 & c & $\mathrm{T}$ & SNP & intergenic & & HP1582 & jhp1488 \\
\hline 547 & 214663 & CGAGAGAGAGAGAGAG & CGAGAGAGAGAGAG & indel & intergenic & & HPG27_8 & \\
\hline 547 & 232964 & c & A & SNP & intergenic & & HPPC_00115 & \\
\hline 547 & 251374 & $G$ & A & $\begin{array}{l}\mathrm{SNP} \\
\end{array}$ & nonsynonymous & HP0045 & & \\
\hline 547 & 276989 & G & A & SNP & nonsynonymous & HPP12_0070 & & \\
\hline 547 & 303813 & A & $\mathrm{T}$ & SNP & nonsynonymous & HPP12_0096 & & \\
\hline 547 & 374756 & CGGGGGGGGGGG & CGGGGGGGGGGGGGG & indel & frameshift insertion & jhp0151 & & \\
\hline 547 & 424517 & CGGGG & CGGGGG & indel & intergenic & & HP0189 & HP0188 \\
\hline s47 & 430922 & G & A & SNP & nonsynonymous & HP0194 & & \\
\hline 547 & 546511 & CAAAAAAAAAAAA & CAAAAAAAAAAAAA & indel & intergenic & & HP1243/HPG27_298 & \\
\hline 547 & 548941 & c & $T$ & SNP & intergenic & & jhp1163 & HPG27_298 \\
\hline 547 & 548965 & c & $\mathrm{T}$ & SNP & intergenic & & jhp1163 & HPG27_298 \\
\hline 547 & 548978 & G & $\mathrm{T}$ & SNP & intergenic & & jhp1163 & HPG27_298 \\
\hline$\$ 47$ & 548994 & A & $T$ & SNP & intergenic & & jhp1163 & HPG27_298 \\
\hline$\$ 47$ & 691071 & G & A & SNP & synonymous & HP0489 & & \\
\hline 547 & 691074 & A & $\mathrm{c}$ & $\begin{array}{ll}\text { SNP } \\
\end{array}$ & synonymous & HP0489 & & \\
\hline$\$ 47$ & 691077 & G & A & SNP & nonsynonymous & HP0489 & & \\
\hline 547 & 691089 & A & G & SNP & synonymous & HP0489 & & \\
\hline 547 & 691091 & c & A & SNP & nonsynonymous & HP0489 & & \\
\hline 547 & 691101 & $T$ & $\mathrm{c}$ & SNP & synonymous & HP0489 & & \\
\hline$\$ 47$ & 691113 & G & $T$ & SNP & nonsynonymous & HP0489 & & \\
\hline 547 & 691173 & c & A & SNP & synonymous & HP0489 & & \\
\hline 547 & 691175 & A & $G$ & SNP & nonsynonymous & HP0489 & & \\
\hline 547 & 691179 & $\mathrm{c}$ & G & SNP & synonymous & HP0489 & & \\
\hline 547 & 691185 & A & $G$ & SNP & nonsynonymous & HP0489 & & \\
\hline 547 & 691189 & c & G & SNP & nonsynonymous & HP0489 & & \\
\hline$\$ 47$ & 691190 & A & c & SNP & nonsynonymous & HP0489 & & \\
\hline 547 & 801836 & GAAAAAAAAAAAA & GAAAAAAAAAAAAA & indel & intergenic & & jhp0540 & \\
\hline 547 & 830053 & C & $\mathrm{T}$ & SNP & nonsynonymous & HPP12_0617 & & \\
\hline 547 & 1043704 & GTTTTTTTTTTTTTT & GтTTTTTTTTTTTTTTTTT, GтTTTTTTTTTTTTTTTT & indel & frameshift insertion/intergenic & HPnc4170 & HP0811 & HPnc4160 \\
\hline 547 & 1187376 & АТTTTTTT & АТTTTTTTT & indel & intergenic & & HP0947 & \\
\hline 547 & 1262251 & c & A & SNP & nonsynonymous & jhp0981 & & \\
\hline 547 & 1295885 & GATATATATATATATATAT & GATATATATATATATATATATAT & indel & intergenic & & HPATCC43504_01238 & \\
\hline 547 & 1332690 & $\mathrm{c}$ & A & SNP & synonymous & HPATCC43504_01275 & & \\
\hline 547 & 1332888 & $\mathrm{~T}$ & G & SNP & synonymous & HPATCC43504_01275 & & \\
\hline 547 & 1332893 & $\mathrm{~T}$ & G & SNP & nonsynonymous & HPATCC43504_01275 & & \\
\hline 547 & 1332900 & G & GT & indel & frameshift deletion & HPATCC43504_01275 & & \\
\hline 547 & 1332906 & c & $\mathrm{T}$ & SNP & synonymous & HPATCC43504_01275 & & \\
\hline 547 & 1332954 & $T$ & G & SNP & synonymous & HPATCC43504_01275 & & \\
\hline 547 & 1332987 & $T$ & $\mathrm{c}$ & $\begin{array}{ll}\text { SNP } \\
\end{array}$ & synonymous & HPATCC43504_01275 & & \\
\hline$\$ 47$ & 1332996 & $T$ & $G$ & SNP & synonymous & HPATCC43504_01275 & & \\
\hline 547 & 1332997 & G & $\mathrm{c}$ & SNP & nonsynonymous & HPATCC43504_01275 & & \\
\hline$\$ 47$ & 1332999 & $T$ & c & SNP & synonymous & HPATCC43504_01275 & & \\
\hline$\$ 47$ & 1582071 & AATT & AATTATT & indel & intergenic & & HELPY_1317 & HP0228 \\
\hline$\$ 48$ & 99327 & c & $T$ & SNP & synonymous & jhp0935 & & \\
\hline 548 & 168379 & c & $T$ & SNP & synonymous & HP1547 & & \\
\hline 548 & 199597 & c & $\mathrm{T}$ & SNP & intergenic & & HP1582 & jhp1488 \\
\hline 548 & 251374 & G & A & SNP & nonsynonymous & HP0045 & & \\
\hline$\$ 48$ & 276989 & $G$ & A & SNP & nonsynonymous & HPP12_0070 & & \\
\hline 548 & 424517 & CGGGG & CGGGGG & indel & intergenic & & HP0189 & HP0188 \\
\hline$\$ 48$ & 430922 & G & A & SNP & nonsynonymous & HP0194 & & \\
\hline 548 & 546511 & CAAAAAAAAAAAA & CAAAAAAAAAAAAA & indel & intergenic & & HP1243/HPG27_298 & \\
\hline 548 & 548965 & c & $T$ & SNP & intergenic & & jhp1163 & HPG27_298 \\
\hline 548 & 548978 & G & $\mathrm{T}$ & SNP & intergenic & & jhp1163 & HPG27_298 \\
\hline$\$ 48$ & 691071 & G & A & SNP & synonymous & HP0489 & & \\
\hline 548 & 691074 & A & $\mathrm{c}$ & SNP & synonymous & HP0489 & & \\
\hline$\$ 48$ & 691077 & G & A & SNP & nonsynonymous & HP0489 & & \\
\hline$\$ 48$ & 691089 & A & G & SNP & synonymous & HP0489 & & \\
\hline
\end{tabular}


bioRxiv preprint doi: https://doi.org/10.1101/2020.02.15.950279; this version posted February 21, 2020. The copyright holder for this preprint (which was not certified by peer review) is the author/funder. All rights reserved. No reuse allowed without permission.

\begin{tabular}{|c|c|c|c|c|c|c|c|c|}
\hline 548 & 691091 & $\mathrm{c}$ & A & SNP & nonsynonymous & HP0489 & & \\
\hline$\$ 48$ & 691101 & $T$ & c & SNP & synonymous & HP0489 & & \\
\hline 548 & 691113 & $G$ & $\mathrm{~T}$ & SNP & nonsynonymous & HP0489 & & \\
\hline$\$ 48$ & 691149 & G & c & SNP & synonymous & HP0489 & & \\
\hline 548 & 691173 & $\mathrm{c}$ & $\mathrm{A}$ & SNP & synonymous & HP0489 & & \\
\hline 548 & 691175 & A & G & SNP & nonsynonymous & HP0489 & & \\
\hline$\$ 48$ & 691179 & c & G & SNP & synonymous & HP0489 & & \\
\hline 548 & 691185 & A & G & SNP & nonsynonymous & HP0489 & & \\
\hline$\$ 48$ & 691189 & $\mathrm{c}$ & $\mathrm{G}$ & SNP & nonsynonymous & HP0489 & & \\
\hline 548 & 691190 & A & $\mathrm{c}$ & SNP & nonsynonymous & HP0489 & & \\
\hline S48 & 830053 & $\mathrm{c}$ & $\mathrm{T}$ & SNP & nonsynonymous & HPP12_0617 & & \\
\hline$\$ 48$ & 958702 & CAAAAAAAAAAAAAAAA & CAAAAAAAAAAAAAAAAAA & indel & intergenic & & HPG27_680 & \\
\hline 548 & 1102177 & A & $\mathrm{T}$ & SNP & intergenic & & HP0876 & HP0875 \\
\hline 548 & 1187376 & АТTTTTTT & АТTTTTTTT & indel & intergenic & & HP0947 & \\
\hline 548 & 1262251 & $\mathrm{c}$ & A & SNP & nonsynonymous & jhp0981 & & \\
\hline$\$ 48$ & 1295885 & GATATATATATATATATAT & $\begin{array}{l}\text { GATATATATATATATATATATAT,GATATATATATA } \\
\text { TATATATAT }\end{array}$ & indel & intergenic & & HPATCC43504_01238 & \\
\hline$\$ 48$ & 1318527 & c & A & SNP & intergenic & jhp1031 & & \\
\hline S48 & 1332690 & $\mathrm{c}$ & A & SNP & synonymous & HPATCC43504_01275 & & \\
\hline 548 & 1332906 & $\mathrm{c}$ & $\mathrm{T}$ & SNP & synonymous & HPATCC43504_01275 & & \\
\hline 548 & 1332954 & $T$ & $\mathrm{G}$ & SNP & synonymous & HPATCC43504_01275 & & \\
\hline$\$ 48$ & 1332987 & $T$ & $\mathrm{c}$ & SNP & synonymous & HPATCC43504_01275 & & \\
\hline s48 & 1332996 & $T$ & G & SNP & synonymous & HPATCC43504_01275 & & \\
\hline 548 & 1332997 & $G$ & $\mathrm{c}$ & SNP & nonsynonymous & HPATCC43504_01275 & & \\
\hline 548 & 1332999 & $\mathrm{~T}$ & $\mathrm{c}$ & SNP & synonymous & HPATCC43504_01275 & & \\
\hline 548 & 1482848 & $G$ & $T$ & SNP & nonsynonymous & HPG27_298 & & \\
\hline 548 & 1482850 & $T$ & G & SNP & nonsynonymous & HPG27_298 & & \\
\hline 548 & 1482859 & $T$ & $\mathrm{c}$ & SNP & synonymous & HPG27_298 & & \\
\hline 548 & 1482860 & c & A & SNP & nonsynonymous & HPG27_298 & & \\
\hline 548 & 1482861 & $\mathrm{G}$ & A & SNP & nonsynonymous & HPG27_298 & & \\
\hline S48 & 1483092 & $\mathrm{G}$ & A & SNP & nonsynonymous & HPG27_298 & & \\
\hline$S 48$ & 1483202 & $G$ & $\mathrm{~A}$ & SNP & nonsynonymous & HPG27_298 & & \\
\hline 548 & 1483269 & A & $G$ & SNP & nonsynonymous & HPG27_298 & & \\
\hline S48 & 1592784 & ATGTG & ATG & indel & frameshift deletion & HP1354 & & \\
\hline$\$ 48$ & 1593602 & TGGGGGGGGGGG & TGGGGGGGGGGGG & indel & frameshift insertion & HP1354 & & \\
\hline 549 & 212868 & c & A & SNP & nonsynonymous & HPG27_8 & & \\
\hline 549 & 214663 & CGAGAGAGAGAGAGAG & CGAGAGAGAGAGAGAGAG & indel & intergenic & & HPG27_8 & \\
\hline 549 & 232964 & $\mathrm{c}$ & $\mathrm{A}$ & SNP & intergenic & & HPPC_00115 & \\
\hline$\$ 49$ & 332580 & $T$ & $\mathrm{~A}$ & SNP & synonymous & HP0119 & & \\
\hline 549 & 332598 & c & $T$ & SNP & synonymous & HP0119 & & \\
\hline$\$ 49$ & 332632 & $G$ & A & SNP & nonsynonymous & HP0119 & & \\
\hline 549 & 332653 & $\mathrm{c}$ & $\mathrm{T}$ & SNP & nonsynonymous & HP0119 & & \\
\hline 549 & 332689 & G & A & SNP & nonsynonymous & HP0119 & & \\
\hline 549 & 374756 & CGGGGGGGGGGGG & CGGGGGGGGGGGGG & indel & frameshift insertion & jhp0151 & & \\
\hline 549 & 548978 & $G$ & $T$ & SNP & intergenic & & jhp1163 & HPG27_298 \\
\hline 549 & 701037 & TGGGGGGGG & TGGGGGGG & indel & frameshift deletion & HP0499 & & \\
\hline 549 & 868876 & $T$ & c & SNP & nonsynonymous & HP0646 & & \\
\hline 549 & 956664 & A & G & SNP & synonymous & HPG27_680 & & \\
\hline 549 & 1166858 & $G$ & $\mathrm{~T}$ & SNP & nonsynonymous & HP0929 & & \\
\hline S49 & 1185804 & A & G & SNP & nonsynonymous & HP0946 & & \\
\hline S49 & 1284699 & AGGGGGGGGGG & AGGGGGGGG & indel & frameshift deletion & HPG27_1018 & & \\
\hline 549 & 1636693 & TGGGG & TGGGGG & indel & frameshift insertion & HPATCC43504_01561 & & \\
\hline 550 & 212868 & $\mathrm{c}$ & A & SNP & nonsynonymous & HPG27_8 & & \\
\hline 550 & 332580 & $\mathrm{~T}$ & A & SNP & synonymous & HP0119 & & \\
\hline 550 & 332632 & $G$ & A & SNP & nonsynonymous & HP0119 & & \\
\hline 550 & 332653 & $\mathrm{c}$ & $\mathrm{T}$ & SNP & nonsynonymous & HP0119 & & \\
\hline$\$ 50$ & 332689 & $G$ & A & SNP & nonsynonymous & HP0119 & & \\
\hline 550 & 464294 & A & G & SNP & intergenic & & HP0226 & \\
\hline S50 & 546511 & CAAAAAAAAAAAA & CAAAAAAAAAAAAA & indel & intergenic & & HP1243/HPG27_298 & \\
\hline $\mathrm{S} 50$ & 548978 & $G$ & $\mathrm{~T}$ & SNP & intergenic & & jhp1163 & HPG27_298 \\
\hline 550 & 701037 & TGGGGGGGG & TGGGGGGG & indel & frameshift deletion & HP0499 & & \\
\hline 550 & 801836 & GAAAAAAAAAAAA & GAAAAAAAAAAAAA & indel & intergenic & & jhp0540 & \\
\hline 550 & 868876 & $T$ & $\mathrm{c}$ & SNP & nonsynonymous & HP0646 & & \\
\hline 550 & 873717 & $\mathrm{c}$ & $\mathrm{T}$ & SNP & synonymous & HP0651 & & \\
\hline 550 & 873807 & c & G & SNP & synonymous & HP0651 & & \\
\hline 550 & 873808 & G & A & SNP & synonymous & HP0651 & & \\
\hline 550 & 873809 & A & $\mathrm{c}$ & SNP & synonymous & HP0651 & & \\
\hline 550 & 873813 & $G$ & A & SNP & synonymous & HP0651 & & \\
\hline 550 & 903745 & TAA & TA & indel & frameshift deletion & HPP12_0689 & & \\
\hline 550 & 958702 & CAAAAAAAAAAAAAAAA & CAAAAAAAAAAAAAAAAAA & indel & intergenic & & HPG27_680 & \\
\hline 550 & 1166858 & $G$ & $\mathrm{~T}$ & SNP & nonsynonymous & HP0929 & & \\
\hline 550 & 1187376 & АТTTTTTT & ATTTTTTTT & indel & intergenic & & HP0947 & \\
\hline 550 & 1284512 & c & $\mathrm{T}$ & SNP & synonymous & HPG27_1018 & & \\
\hline 550 & 1284514 & $\mathrm{c}$ & $\mathrm{T}$ & SNP & nonsynonymous & HPG27_1018 & & \\
\hline S50 & 1284517 & $T$ & $G$ & SNP & synonymous & HPG27_1018 & & \\
\hline 550 & 1284553 & c & A & SNP & nonsynonymous & HPG27_1018 & & \\
\hline 550 & 1284699 & AGGGGGGGGGG & AGGGGGGGGG & indel & frameshift deletion & HPG27_1018 & & \\
\hline 550 & 1636693 & TGGGG & TGGGGG & indel & frameshift insertion & HPATCC43504_01561 & & \\
\hline 551 & 212868 & $\mathrm{c}$ & A & SNP & nonsynonymous & HPG27_8 & & \\
\hline 551 & 332580 & $\mathrm{~T}$ & $\mathrm{~A}$ & SNP & synonymous & HP0119 & & \\
\hline 551 & 332598 & $\mathrm{c}$ & $\mathrm{T}$ & SNP & synonymous & HP0119 & & \\
\hline S51 & 332632 & $G$ & A & SNP & nonsynonymous & HP0119 & & \\
\hline S51 & 332653 & c & $T$ & SNP & nonsynonymous & HP0119 & & \\
\hline S51 & 332689 & $G$ & A & SNP & nonsynonymous & HP0119 & & \\
\hline S51 & 374756 & CGGGGGGGGGGGG & CGGGGGGGGGGGGGG & indel & frameshift insertion & jhp0151 & & \\
\hline S51 & 546511 & CAAAAAAAAAAAA & CAAAAAAAAAAAAA & indel & intergenic & & HP1243/HPG27_298 & \\
\hline S51 & 548978 & G & $\mathrm{T}$ & SNP & intergenic & & jhp1163 & HPG27_298 \\
\hline
\end{tabular}




\begin{tabular}{|c|c|c|c|c|c|c|c|c|}
\hline 551 & 701037 & TGGGGGGGG & TGGGGGGG & indel & frameshift deletion & HP0499 & & \\
\hline$\$ 51$ & 801836 & GAAAAAAAAAAAA & GAAAAAAAAAAAAA & indel & intergenic & & jhp0540 & \\
\hline S51 & 839999 & $T$ & c & SNP & nonsynonymous & $\begin{array}{ll}\text { HPB8_818 } \\
\end{array}$ & & \\
\hline 551 & 868876 & $\mathrm{~T}$ & $\mathrm{c}$ & SNP & nonsynonymous & HP0646 & & \\
\hline 551 & 958719 & G & A & SNP & intergenic & & HPG27_680 & \\
\hline S51 & 1043704 & GTTTTTTTTTTTTT & GтTTTTTाTTTTTT & indel & frameshift insertion/intergenic & HPnc4170 & HP0811 & HPnc4160 \\
\hline 551 & 1166858 & G & $T$ & SNP & nonsynonymous & HP0929 & & \\
\hline S51 & 1636693 & TGGGG & TGGGGG & indel & frameshift insertion & HPATCC43504_01561 & & \\
\hline$\$ 52$ & 212868 & c & A & SNP & nonsynonymous & HPG27_8 & & \\
\hline$\$ 52$ & 232963 & A & c & SNP & intergenic & & HPPC_00115 & \\
\hline 552 & 232964 & $\mathrm{c}$ & A & SNP & intergenic & & $\begin{array}{ll}\text { HPPC_00115 } \\
\end{array}$ & \\
\hline$\$ 52$ & 332580 & $T$ & A & SNP & synonymous & HP0119 & & \\
\hline 552 & 332598 & $c$ & T & SNP & synonymous & HР0119 & & \\
\hline 552 & 332632 & G & A & SNP & nonsynonymous & HP0119 & & \\
\hline 552 & 332653 & c & $T$ & SNP & nonsynonymous & $\begin{array}{ll}\text { HP0119 } \\
\end{array}$ & & \\
\hline 552 & 332689 & $G$ & A & SNP & nonsynonymous & $\begin{array}{ll}\text { HP0119 } \\
\end{array}$ & & \\
\hline 552 & 374756 & CGGGGGGGGGGGG & CGGGGGGGGGGGGGG & indel & frameshift insertion & jhp0151 & & \\
\hline 552 & 548965 & c & $T$ & SNP & intergenic & & jhp1163 & HPG27_298 \\
\hline 552 & 548978 & G & $T$ & SNP & intergenic & & jhp1163 & $\begin{array}{l}\text { HPG27_298 } \\
\end{array}$ \\
\hline 552 & 701037 & TGGGGGGGG & TGGGGGGG & indel & frameshift deletion & $\begin{array}{ll}\text { HP0499 } \\
\end{array}$ & & \\
\hline 552 & 801836 & GAAAAAAAAAAAA & GAAAAAAAAAAAAA & indel & intergenic & & jhp0540 & \\
\hline 552 & 839999 & $T$ & c & SNP & nonsynonymous & $\begin{array}{ll}\text { HPB8_818 } \\
\end{array}$ & & \\
\hline 552 & 868876 & $T$ & $\mathrm{c}$ & SNP & nonsynonymous & $\begin{array}{ll}\text { HP0646 } \\
\end{array}$ & & \\
\hline 552 & 1043704 & GтTTTTTTTTTTTTT & GтTтTTTTाTाTTT & indel & frameshift insertion/intergenic & HPnc4170 & HP0811 & HPnc4160 \\
\hline 552 & 1166858 & G & $T$ & SNP & nonsynonymous & HP0929 & & \\
\hline$\$ 52$ & 1187376 & АТTTTTTT & АТTTTTTTT & indel & intergenic & & HР0947 & \\
\hline$\$ 52$ & 1636693 & \begin{tabular}{c|c|c|} 
TGGGG \\
\end{tabular} & TGGGGG & indel & frameshift insertion & HPATCC43504_01561 & & \\
\hline 552 & 1663529 & GTTTTT & GTाT & indel & 1 bp deletion & & & \\
\hline 553 & 658 & c & $T$ & SNP & synonymous & $\begin{array}{ll}\text { HP1529 } \\
\text { HP } 15\end{array}$ & & \\
\hline 553 & 214663 & CGAGAGAGAGAGAGAG & CGAGAGAGAGAGAGAGAG & indel & intergenic & & HPG 27_8 & \\
\hline 553 & 232964 & c & A & SNP & intergenic & & HPPC_00115 & \\
\hline 553 & 303813 & A & $T$ & SNP & nonsynonymous & $\begin{array}{ll}\text { HPP12_0096 } \\
\end{array}$ & & \\
\hline 553 & 303816 & A & $T$ & $\begin{array}{l}\text { SNP } \\
\end{array}$ & nonsynonymous & $\begin{array}{ll}\text { HPP12_0096 } \\
\end{array}$ & & \\
\hline 553 & 303827 & CGGGGGGGGGGGG & G & indel & frameshift deletion & $\begin{array}{ll}\text { HPP12_0096 } \\
\end{array}$ & & \\
\hline 553 & 315327 & ACCCCCCCCCC & ACCCCCCCCCCC & indel & intergenic & & $\begin{array}{ll}\text { HP0103 } \\
\end{array}$ & \\
\hline 553 & 374756 & CGGGGGGGGGGGG & CGGGGGGGGGGGGGG & indel & frameshift insertion & jhp0151 & & \\
\hline 553 & 431032 & c & $T$ & SNP & synonymous & HP0194 & & \\
\hline 553 & 437765 & G & c & SNP & intergenic & & HP0204 & HELPY_0206 \\
\hline 553 & 443932 & АСТСТСТСТСТСТСТСТСТС & АСТСТСТСТСТСТСТСТС & indel & frameshift deletion & $\begin{array}{ll}\text { HPPC_01040 } \\
\end{array}$ & & \\
\hline 553 & 450628 & $G^{G}$ & A & SNP & synonymous & HP0213 & & \\
\hline 553 & 546115 & $\begin{array}{l}\text { АATTTAATCTTATTTAATCTTATTTAATCTTATTTAA } \\
\text { TCTTATTTAATCTATTTAATCT }\end{array}$ & 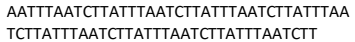 & indel & intergenic & & HP1243/HPG27_298 & \\
\hline 553 & 546511 & CAAAAAAAAAAAA & CAAAAAAAAAAAAA & indel & intergenic & & HP1243/HPG27_298 & \\
\hline 553 & 548978 & 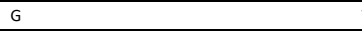 & $T$ & $\begin{array}{ll}\text { SNP } \\
\end{array}$ & intergenic & & jhp1163 & HPG27_298 \\
\hline 553 & 620169 & $\mathrm{c}$ & $T$ & SNP & nonsynonymous & HPAG1_1119 & & \\
\hline 553 & 626404 & c & $\mathrm{T}$ & SNP & synonymous & HP1175 & & \\
\hline 553 & 627243 & G & A & SNP & nonsynonymous & HP1174 & & \\
\hline 553 & 701041 & G & A & SNP & nonsynonymous & HP0499 & & \\
\hline 553 & 801836 & GAAAAAAAAAAAA & GAAAAAAAAAAAAA & indel & intergenic & & jhp0540 & \\
\hline 553 & 839999 & $T$ & c & 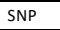 & nonsynonymous & HPB8_818 & & \\
\hline 553 & 1187376 & АТTTTTTT & АТTTTTTTT & indel & intergenic & & $\begin{array}{ll}\text { HP0947 } \\
\end{array}$ & \\
\hline 553 & 1319683 & G & c & SNP & nonsynonymous & jhp1032 & & \\
\hline 553 & 1592784 & ATGTG & ATG & indel & frameshift deletion & HP1354 & & \\
\hline 553 & 1592801 & $T$ & G & SNP & synonymous & HP1354 & & \\
\hline 553 & 1663529 & GTTTTT & GTाT & indel & 1 bp deletion & & & \\
\hline 553 & 1669898 & c & $T$ & SNP & synonymous & HP1450 & & \\
\hline 554 & 658 & $\mathrm{c}$ & $T$ & $\begin{array}{l}\text { SNP } \\
\end{array}$ & synonymous & $\begin{array}{ll}\text { HP1529 } \\
\text { HP }\end{array}$ & & \\
\hline 554 & 214663 & CGAGAGAGAGAGAGAG & CGAGAGAGAGAGAGAGAG & indel & intergenic & & HPG 27_8 & \\
\hline 554 & 431032 & c & $T$ & SNP & synonymous & HP0194 & & \\
\hline 554 & 437765 & G & c & SNP & intergenic & & $\begin{array}{ll}\text { HP0204 } \\
\end{array}$ & $\begin{array}{l}\text { HELPY_0206 } \\
\text { HEL }\end{array}$ \\
\hline 554 & 450628 & G & A & SNP & synonymous & HP0213 & & \\
\hline s54 & 546115 & $\begin{array}{l}\text { AATTTAATCTTATTTAATCTTATTTAATCTTATTTAA } \\
\text { TCTTATTTAATCTATTTAATCTT }\end{array}$ & $\begin{array}{l}\text { AАTTTAATCTTATTTAATCTTATTTAATCTTATTTAA } \\
\text { TCTTATTTAACTATTTAACTATTTAATCTT }\end{array}$ & indel & intergenic & & HP1243/HPG27_298 & \\
\hline 554 & 546511 & CAAAAAAAAAAAA & CAAAAAAAAAAAAa & indel & intergenic & & HP1243/HPG27_298 & \\
\hline 554 & 620169 & c & $T$ & SNP & nonsynonymous & HPAG1_1119 & & \\
\hline 554 & 626404 & c & $T$ & SNP & synonymous & HP1175 & & \\
\hline 554 & 627243 & $G$ & A & $\begin{array}{l}\text { SNP } \\
\end{array}$ & nonsynonymous & $\begin{array}{ll}\text { HP1174 } \\
\end{array}$ & & \\
\hline 554 & 701041 & G & A & $\begin{array}{l}\text { SNP } \\
\end{array}$ & nonsynonymous & $\begin{array}{ll}\text { HP0499 } \\
\end{array}$ & & \\
\hline 554 & 1187376 & АТTTTTTT & АТTTाTTTT & indel & intergenic & & $\begin{array}{ll}\text { HP0947 } \\
\end{array}$ & \\
\hline 554 & 1319683 & G & c & SNP & nonsynonymous & jhp1032 & & \\
\hline 554 & 1592785 & TGTGG & TG & indel & frameshift deletion & HP1354 & & \\
\hline S54 & 1669898 & c & $T$ & SNP & synonymous & HP1450 & & \\
\hline 555 & 658 & $\mathrm{c}$ & $T$ & SNP & synonymous & $\begin{array}{ll}\text { HP1529 } \\
\end{array}$ & & \\
\hline$\$ 55$ & 214663 & CGAGAGAGAGAGAGAG & CGAGAGAGAGAGAGAGAG & indel & intergenic & & HPG 27_8 & \\
\hline 555 & 315327 & ACCCCCCCCCCC & AсCCCCCCCCCCC & indel & intergenic & & HP0103 & \\
\hline$\$ 55$ & 431032 & c & $T$ & SNP & synonymous & HP0194 & & \\
\hline 555 & 437765 & G & c & SNP & intergenic & & $\begin{array}{ll}\text { HP0204 } \\
\end{array}$ & HELPY_0206 \\
\hline 555 & 450628 & G & A & SNP & synonymous & HP0213 & & \\
\hline 555 & 455599 & TGGGGGGGGGG & $\begin{array}{l}\text { TGGGGGGGGGGG } \\
\end{array}$ & indel & frameshift insertion & $\begin{array}{ll}\text { HP0217 } \\
\end{array}$ & & \\
\hline 555 & 546115 & $\begin{array}{l}\text { AATTAAATCTATTTAATCTTATTTAATCTATTTAA } \\
\text { TCTAATTAATCTATTAACCT }\end{array}$ & $\begin{array}{l}\text { AATTAATCTTATTAAATCTTATTTAATCTATTTAA } \\
\text { TCTATTTAATCTATTIAACTTATTAATCTT }\end{array}$ & indel & intergenic & & HP1243/HPG27_298 & \\
\hline 555 & 548957 & c & $T$ & SNP & intergenic & & jhp1163 & HPG27_298 \\
\hline 555 & 548965 & c & $T$ & SNP & intergenic & & jhp1163 & HPG27_298 \\
\hline$\$ 55$ & 620169 & c & $T$ & SNP & nonsynonymous & HPAG1_1119 & & \\
\hline 555 & 626404 & c & $T$ & SNP & synonymous & HP1175 & & \\
\hline$\$ 55$ & 627243 & G & A & SNP & nonsynonymous & HP1174 & & \\
\hline 555 & 701041 & G & A & SNP & nonsynonymous & HP0499 & & \\
\hline 555 & 801836 & GAAAAAAAAAAAA & GAAAAAAAAAAAAA & indel & intergenic & & jhp0540 & \\
\hline
\end{tabular}




\begin{tabular}{|c|c|c|c|c|c|c|c|c|}
\hline 555 & 839999 & $\mathrm{~T}$ & c & SNP & nonsynonymous & HPB8_818 & & \\
\hline S55 & 958702 & CAAAAAAAAAAAAAAAAA & CAAAAAAAAAAAAAAAAAAAA & indel & intergenic & & HPG27_680 & \\
\hline 555 & 1043704 & GTTTTTTTTTTTTTT & GTTTTTTTTTTTTTTTT & indel & frameshift insertion/intergenic & HPnc4170 & HP0811 & HPnc4160 \\
\hline 555 & 1187376 & АТTTTTTT & АТTTTTTTT & indel & intergenic & & HP0947 & \\
\hline 555 & 1192576 & G & $\mathrm{T}$ & SNP & intergenic & & HP0953 & \\
\hline 555 & 1319683 & G & c & SNP & nonsynonymous & jhp1032 & & \\
\hline 555 & 1592784 & ATGTG & ATG & indel & frameshift deletion & HP1354 & & \\
\hline 555 & 1593602 & TGGGGGGGGGGG & TGGGGGGGGGGGG & indel & frameshift insertion & HP1354 & & \\
\hline 555 & 1669898 & c & $\mathrm{T}$ & SNP & synonymous & HP1450 & & \\
\hline 556 & 658 & c & T & SNP & synonymous & HP1529 & & \\
\hline 556 & 214663 & CGAGAGAGAGAGAGAG & CGAGAGAGAGAGAGAGAG & indel & intergenic & & HPG27_8 & \\
\hline S56 & 315327 & AсcCCCCCCCCC & AсCCCCCCCCCC & indel & intergenic & & HP0103 & \\
\hline 556 & 374756 & CGGGGGGGGGGGG & CGGGGGGGGGGGGGG & indel & frameshift insertion & jhp0151 & & \\
\hline 556 & 431032 & c & $T$ & SNP & synonymous & HP0194 & & \\
\hline 556 & 437765 & G & c & SNP & intergenic & & HP0204 & HELPY_0206 \\
\hline 556 & 443932 & АСТСТСТстстстстстСТС & АСТСТСТстСТСТСТСТС & indel & frameshift deletion & HPPC_01040 & & \\
\hline S56 & 450628 & G & A & SNP & synonymous & HP0213 & & \\
\hline 556 & 455599 & TGGGGGGGGGG & TGGGGGGGGGGG & indel & frameshift insertion & HP0217 & & \\
\hline S56 & 546115 & $\begin{array}{l}\text { AATTTAATCTTATTTAATCTTATTTAATCTTATTTAA } \\
\text { TCTTATTTAATCTTATTTAATCTT }\end{array}$ & $\begin{array}{l}\text { AATTTAATCTTATTTAATCTTATTTAATCTTATTTAA } \\
\text { TCTTATTTAATCTTATTTAATCTTATTTAATCTT }\end{array}$ & indel & intergenic & & HP1243/HPG27_298 & \\
\hline 556 & 546511 & CAAAAAAAAAAAA & CAAAAAAAAAAAAA & indel & intergenic & & HP1243/HPG27_298 & \\
\hline$\$ 56$ & 548978 & G & $\mathrm{T}$ & SNP & intergenic & & jhp1163 & HPG27_298 \\
\hline S56 & 548986 & СТTTTTTT & СТTTTTTTT & indel & intergenic & & jhp1163 & HPG27_298 \\
\hline 556 & 620169 & c & $\mathrm{T}$ & SNP & nonsynonymous & HPAG1_1119 & & \\
\hline 556 & 626404 & c & $\mathrm{T}$ & SNP & synonymous & HP1175 & & \\
\hline 556 & 627243 & $G$ & A & SNP & nonsynonymous & HP1174 & & \\
\hline 556 & 701041 & G & A & SNP & nonsynonymous & HP0499 & & \\
\hline 556 & 801836 & GAAAAAAAAAAAA & GAAAAAAAAAAAAA & indel & intergenic & & jhp0540 & \\
\hline S56 & 1043704 & GTTTTTTTTTTTTTT & GTTTTTTTTTTTTTTTTTT & indel & frameshift insertion/intergenic & HPnc4170 & HP0811 & HPnc4160 \\
\hline 556 & 1124346 & CGAGAGAGAGAGAGAGAGAG & CGAGAGAGAGAGAGAGAG & indel & intergenic & & HPG27_1187 & \\
\hline 556 & 1302389 & G & A & SNP & synonymous & HP0364 & & \\
\hline 556 & 1319683 & G & c & SNP & nonsynonymous & jhp1032 & & \\
\hline S56 & 1410493 & G & A & SNP & nonsynonymous & jhp0373 & & \\
\hline 556 & 1592785 & TGTGG & TG & indel & frameshift deletion & HP1354 & & \\
\hline$\$ 56$ & 1669898 & c & $\mathrm{T}$ & SNP & synonymous & HP1450 & & \\
\hline 557 & 658 & c & $\mathrm{T}$ & SNP & synonymous & HP1529 & & \\
\hline 557 & 214663 & CGAGAGAGAGAGAGAG & CGAGAGAGAGAGAGAGAG & indel & intergenic & & HPG27_8 & \\
\hline 557 & 374756 & CGGGGGGGGGGG & CGGGGGGGGGGGGGG & indel & frameshift insertion & jhp0151 & & \\
\hline 557 & 431032 & c & $\mathrm{T}$ & SNP & synonymous & HP0194 & & \\
\hline 557 & 437765 & G & $\mathrm{c}$ & SNP & intergenic & & HP0204 & HELPY_0206 \\
\hline 557 & 450628 & G & A & SNP & synonymous & HP0213 & & \\
\hline 557 & 546524 & c & A & SNP & intergenic & & HP1243/HPG27_298 & \\
\hline 557 & 607390 & G & A & SNP & synonymous & jhp1115 & & \\
\hline 557 & 620169 & c & $\mathrm{T}$ & SNP & nonsynonymous & HPAG1_1119 & & \\
\hline 557 & 626404 & c & $\mathrm{T}$ & SNP & synonymous & HP1175 & & \\
\hline 557 & 627243 & G & A & SNP & nonsynonymous & HP1174 & & \\
\hline 557 & 701041 & G & A & SNP & nonsynonymous & HP0499 & & \\
\hline 557 & 748416 & $T$ & A & SNP & intergenic & & HP0514 & \\
\hline 557 & 801836 & GAAAAAAAAAAAA & GAAAAAAAAAAAAA & indel & intergenic & & jhp0540 & \\
\hline 557 & 958575 & GAGA & GAGAAAGA & indel & intergenic & & HPG27_680 & \\
\hline 557 & 1124346 & CGAGAGAGAGAGAGAGAGAG & CGAGAGAGAGAGAGAGAGAGAG & indel & intergenic & & HPG27_1187 & \\
\hline 557 & 1187376 & АТTTTTTT & ATTTTTTTT & indel & intergenic & & HP0947 & \\
\hline 557 & 1272391 & G & A & SNP & nonsynonymous & & & \\
\hline 557 & 1284699 & AGGGGGGGGGG & AGGGGGGGGG & indel & frameshift deletion & HPG27_1018 & & \\
\hline 557 & 1555634 & $T$ & c & SNP & nonsynonymous & HELPY_0259 & & \\
\hline 557 & 1582072 & ATT & ATTTTT & indel & intergenic & & HELPY_1317 & HP0228 \\
\hline 557 & 1592786 & GT & G & indel & frameshift deletion & HP1354 & & \\
\hline 557 & 1592801 & $T$ & G & SNP & synonymous & HP1354 & & \\
\hline 557 & 1593602 & TGGGGGGGGGGG & G & indel & frameshift insertion & HP1354 & & \\
\hline 557 & 1669898 & c & $\mathrm{T}$ & SNP & synonymous & HP1450 & & \\
\hline 558 & 658 & c & $\mathrm{T}$ & SNP & synonymous & HP1529 & & \\
\hline 558 & 961 & G & A & SNP & nonsynonymous & HP1529 & & \\
\hline 558 & 214663 & CGAGAGAGAGAGAGAG & CGAGAGAGAGAGAGAGAG & indel & intergenic & & HPG27_8 & \\
\hline 558 & 232963 & A & c & SNP & intergenic & & HPPC_00115 & \\
\hline 558 & 232964 & c & A & SNP & intergenic & & HPPC_00115 & \\
\hline S58 & 315327 & ACCCCCCCCCCC & ACCCCCCCCCCCC & indel & intergenic & & HP0103 & \\
\hline 558 & 374756 & CGGGGGGGGGGGG & CGGGGGGGGGGGGGG & indel & frameshift insertion & jhp0151 & & \\
\hline 558 & 431032 & c & $\mathrm{T}$ & SNP & synonymous & HP0194 & & \\
\hline 558 & 437765 & $G$ & c & SNP & intergenic & & HP0204 & HELPY_0206 \\
\hline 558 & 450628 & G & A & SNP & synonymous & HP0213 & & \\
\hline 558 & 455599 & TGGGGGGGGGG & TGGGGGGGGGGG & indel & frameshift insertion & HP0217 & & \\
\hline 558 & 546115 & $\begin{array}{l}\text { AATTTAATCTTATTTAATCTTATTTAATCTTATTTAA } \\
\text { TCTTATTTAATCTTATTTAATCTT }\end{array}$ & $\begin{array}{l}\text { AATTTAATCTTATTTAATCTTATTTAATCTTATTTAA } \\
\text { TCTTATTTAATCTTATTTAATCTTATTTAATCTTATT } \\
\text { TAATCTT }\end{array}$ & indel & intergenic & & HP1243/HPG27_298 & \\
\hline 558 & 546511 & CAAAAAAAAAAAA & CAAAAAAAAAAAAA & indel & intergenic & & HP1243/HPG27_298 & \\
\hline 558 & 548965 & c & $T$ & SNP & intergenic & & jhp1163 & HPG27_298 \\
\hline 558 & 620169 & c & $T$ & SNP & nonsynonymous & HPAG1_1119 & & \\
\hline 558 & 626404 & c & $\mathrm{T}$ & SNP & synonymous & HP1175 & & \\
\hline 558 & 627243 & G & A & SNP & nonsynonymous & HP1174 & & \\
\hline 558 & 701041 & G & $\mathrm{A}$ & SNP & nonsynonymous & HP0499 & & \\
\hline 558 & 801836 & GAAAAAAAAAAAA & GAAAAAAAAAAAAA & indel & intergenic & & jhp0540 & \\
\hline 558 & 805851 & A & $T$ & SNP & intergenic & & HPG27_556 & \\
\hline 558 & 958574 & CGAGAGAGAGAGAG & CGAGAGAGAGAGAGAG & indel & intergenic & & HPG27_680 & \\
\hline 558 & 958702 & CAAAAAAAAAAAAAAAA & CAAAAAAAAAAAAAAAAAAA & indel & intergenic & & HPG27_680 & \\
\hline 558 & 1043704 & GTTTTTTTTTTTTTT & GTTTTTTTTTTTTTTTTT & indel & frameshift insertion/intergenic & HPnc4170 & HP0811 & HPnc4160 \\
\hline $\mathrm{S} 58$ & 1187376 & АТTTTTTT & АТTTTTTTT & indel & intergenic & & HP0947 & \\
\hline 558 & 1284699 & AGGGGGGGGGG & AGGGGGGGGGGG & indel & frameshift deletion & HPG27_1018 & & \\
\hline 558 & 1555634 & $T$ & c & SNP & nonsynonymous & HELPY_0259 & & \\
\hline
\end{tabular}




\begin{tabular}{|c|c|c|c|c|c|c|c|c|}
\hline 558 & 1592784 & ATGTG & ATG & indel & frameshift deletion & HP1354 & & \\
\hline$\$ 58$ & 1593602 & TGGGGGGGGGGG & TGGGGGGGGGGGG & indel & frameshift deletion & HP1354 & & \\
\hline 558 & 1669898 & $\mathrm{c}$ & $\mathrm{T}$ & SNP & synonymous & HP1450 & & \\
\hline 559 & 658 & $\mathrm{c}$ & $T$ & SNP & synonymous & HP1529 & & \\
\hline S59 & 27485 & AGGGGGG & AGGGGGGG & indel & intergenic & & HP1506 & jhp1400 \\
\hline 559 & 431032 & $\mathrm{c}$ & $T$ & SNP & synonymous & HP0194 & & \\
\hline S59 & 437765 & G & c & SNP & intergenic & & HP0204 & HELPY_0206 \\
\hline$\$ 59$ & 450628 & G & A & SNP & synonymous & HP0213 & & \\
\hline$\$ 59$ & 546511 & CAAAAAAAAAAAA & CAAAAAAAAAAAAA & indel & intergenic & & HP1243/HPG27_298 & \\
\hline 559 & $\begin{array}{ll}548978 \\
\end{array}$ & G & $\mathrm{T}$ & SNP & intergenic & & jhp1163 & HPG27_298 \\
\hline 559 & 620169 & $\mathrm{c}$ & $\mathrm{T}$ & SNP & nonsynonymous & $\begin{array}{l}\text { HPAG1_1119 } \\
\end{array}$ & & \\
\hline 559 & 626404 & c & $T$ & SNP & synonymous & HP1175 & & \\
\hline 559 & 627243 & $G$ & A & SNP & nonsynonymous & HP1174 & & \\
\hline 559 & 701041 & G & $\mathrm{A}$ & SNP & nonsynonymous & HP0499 & & \\
\hline$\$ 59$ & 757302 & $G$ & A & SNP & synonymous & $\begin{array}{l}\text { HPATCC43504_00741 } \\
\end{array}$ & & \\
\hline$\$ 59$ & 801836 & GAAAAAAAAAAAA & GAAAAAAAAAAAAA & indel & intergenic & & jhp0540 & \\
\hline S59 & 854528 & $\mathrm{c}$ & $\mathrm{T}$ & SNP & nonsynonymous & HP0632 & & \\
\hline$\$ 59$ & 958574 & CGAGAGAGAGAGAG & CGAGAGAGAGAGAGAG & indel & intergenic & & HPG27_680 & \\
\hline 559 & 1043704 & GтTтTTтTTTTTTT & GттाтाтाтाтाтT & indel & frameshift insertion/intergenic & HPnc4170 & HP0811 & HPnc4160 \\
\hline 559 & 1124346 & CGAGAGAGAGAGAGAGAGAG & CGAGAGAGAGAGAGAGAG & indel & intergenic & & HPG27_1187 & \\
\hline 559 & 1187376 & АТTTTTTT & АТTTाTTTT & indel & intergenic & & HP0947 & \\
\hline 559 & 1284699 & AGGGGGGGGGG & AGGGGGGGGGGG & indel & frameshift deletion & $\begin{array}{l}\text { HPG27_1018 } \\
\end{array}$ & & \\
\hline$\$ 59$ & 1555634 & $T$ & c & SNP & nonsynonymous & HELPY_0259 & & \\
\hline S59 & 1582224 & АстстстстстстстстС & АстСтСТСТСТСТСТС & indel & intergenic & & HELPY_1317 & HPO228 \\
\hline$\$ 59$ & 1592800 & G & GGGT & indel & frameshift insertion & HP1354 & & \\
\hline$\$ 59$ & 1669898 & $\mathrm{c}$ & $T$ & SNP & synonymous & HP1450 & & \\
\hline 560 & 658 & $\mathrm{c}$ & $T$ & SNP & synonymous & HP1529 & & \\
\hline 560 & 214663 & CGAGAGAGAGAGAGAG & CGAGAGAGAGAGAGAGAG & indel & intergenic & & HPG27_8 & \\
\hline 560 & 232963 & A & $\mathrm{c}$ & SNP & intergenic & & $\begin{array}{ll}\text { HPPC_00115 } \\
\end{array}$ & \\
\hline 560 & 232964 & c & A & SNP & intergenic & & HPPC_00115 & \\
\hline 560 & 315327 & AcccccccccC & Accccccccccc & indel & intergenic & & HP0103 & \\
\hline 560 & 345517 & c & $T$ & SNP & synonymous & HP0132 & & \\
\hline 560 & 374756 & CGGGGGGGGGGGG & CGGGGGGGGGGGGGG & indel & frameshift insertion & jhp0151 & & \\
\hline 560 & 431032 & $\mathrm{c}$ & $\mathrm{T}$ & SNP & synonymous & HP0194 & & \\
\hline 560 & 437765 & G & $\mathrm{c}$ & SNP & intergenic & & HP0204 & HELPY_0206 \\
\hline 560 & 450628 & G & A & SNP & synonymous & HP0213 & & \\
\hline 560 & 488821 & c & $\mathrm{T}$ & SNP & nonsynonymous & jhp1233 & & \\
\hline 560 & 546511 & CAAAAAAAAAAAA & CAAAAAAAAAAAAA & indel & intergenic & & HP1243/HPG27_298 & \\
\hline 560 & 620169 & $\mathrm{c}$ & $T$ & SNP & nonsynonymous & $\begin{array}{ll}\text { HPAG1_11199 } \\
\end{array}$ & & \\
\hline 560 & 626404 & $\mathrm{c}$ & $T$ & SNP & synonymous & HP1175 & & \\
\hline 560 & 627243 & G & A & SNP & nonsynonymous & HP1174 & & \\
\hline 560 & 701041 & G & A & SNP & nonsynonymous & HP0499 & & \\
\hline 560 & 801836 & GAAAAAAAAAAAA & GAAAAAAAAAAAAA & indel & intergenic & & jhp0540 & \\
\hline 560 & 958574 & CGAGAGAGAGAGAG & CGAGAGAGAGAGAGAG & indel & intergenic & & HPG27_680 & \\
\hline 560 & 958702 & CAAAAAAAAAAAAAAAA & CAAAAAAAAAAAAAAAAAAA & indel & intergenic & & HPG27_680 & \\
\hline 560 & 1043704 & GтTTTTTTTTTTTTT & GтTTTTTTTTTTTTTTTTT & indel & frameshift insertion/intergenic & HPnc4170 & HP0811 & $\begin{array}{ll}\text { HPnc4160 } \\
\end{array}$ \\
\hline 560 & 1187376 & АТTTTTTT & АТTTाTTTT & indel & intergenic & & $\begin{array}{ll}\text { HP0947 } \\
\end{array}$ & \\
\hline 560 & 1284699 & AGGGGGGGGGG & AGGGGGGGGG & indel & frameshift deletion & HPG27_1018 & & \\
\hline 560 & 1318527 & $\mathrm{c}$ & A & SNP & intergenic & jhp1031 & & \\
\hline 560 & 1555634 & $T$ & c & SNP & nonsynonymous & HELPY_0259 & & \\
\hline 560 & 1592784 & ATGTG & ATG & indel & frameshift deletion & HP1354 & & \\
\hline 560 & 1592801 & $\mathrm{~T}$ & G & SNP & synonymous & HP1354 & & \\
\hline 560 & 1669898 & $\mathrm{c}$ & $\mathrm{T}$ & SNP & synonymous & HP1450 & & \\
\hline 561 & 658 & c & $\mathrm{T}$ & SNP & synonymous & HP1529 & & \\
\hline $\mathrm{S} 61$ & 214663 & CGAGAGAGAGAGAGAG & CGAGAGAGAGAGAGAGAG & indel & intergenic & & HPG27_8 & \\
\hline$S 61$ & 249555 & G & A & SNP & nonsynonymous & HP0044 & & \\
\hline$S 61$ & 315327 & ACCCCCCCCCC & ACCCCCCCCCCC & indel & intergenic & & HP0103 & \\
\hline 561 & 374756 & CGGGGGGGGGGGG & CGGGGGGGGGGGGGG & indel & frameshift insertion & jhp0151 & & \\
\hline 561 & 413866 & A & $G$ & SNP & synonymous & HP1535 & & \\
\hline $\mathrm{S} 61$ & 413887 & A & $G$ & SNP & synonymous & HP1535 & & \\
\hline 561 & 413983 & c & $\mathrm{T}$ & SNP & synonymous & HP1535 & & \\
\hline$S 61$ & 431032 & c & $\mathrm{T}$ & SNP & synonymous & HP0194 & & \\
\hline$S 61$ & 437765 & $G$ & c & SNP & intergenic & & HP0204 & $\begin{array}{l}\text { HELPY_0206 } \\
\end{array}$ \\
\hline$S 61$ & $\begin{array}{ll}450628 \\
4528\end{array}$ & 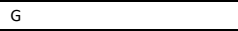 & A & SNP & synonymous & HP0213 & & \\
\hline$S 61$ & 546511 & CAAAAAAAAAAAA & CAAAAAAAAAAAAA & indel & intergenic & & HP1243/HPG27_298 & \\
\hline$S 61$ & 548965 & $\mathrm{c}$ & $\mathrm{T}$ & SNP & intergenic & & jhp1163 & HPG27_298 \\
\hline $\mathrm{S} 61$ & 548978 & G & $\mathrm{T}$ & SNP & intergenic & & jhp1163 & HPG27_298 \\
\hline 561 & 620169 & $\mathrm{c}$ & $\mathrm{T}$ & SNP & nonsynonymous & HPAG1_1119 & & \\
\hline$S 61$ & 626404 & $\mathrm{c}$ & $\mathrm{T}$ & SNP & synonymous & HP1175 & & \\
\hline 561 & 627243 & G & A & SNP & nonsynonymous & HP1174 & & \\
\hline$S 61$ & 701041 & $G$ & A & SNP & nonsynonymous & HP0499 & & \\
\hline 561 & 801836 & GAAAAAAAAAAAA & GAAAAAAAAAAAAAA & indel & intergenic & & jhp0540 & \\
\hline$S 61$ & 839999 & $T$ & c & SNP & nonsynonymous & HPB8_818 & & \\
\hline$S 61$ & 1043704 & GтTTTTTTTTTTTTT & GTTTTTTTTTTTTTTTTTT & indel & frameshift insertion/intergenic & HPnc4170 & HP0811 & HPnc4160 \\
\hline 561 & 1122223 & $\mathrm{c}$ & $\mathrm{T}$ & SNP & intergenic & & HP0895 & \\
\hline 561 & 1122228 & TAAAAA & TAAAAAA & indel & intergenic & & HP0895 & \\
\hline 561 & 1187376 & АТTTTTTT & АТTTTTTTT & indel & intergenic & & HP0947 & \\
\hline $\mathrm{s} 61$ & 1483092 & G & A & SNP & nonsynonymous & HPG27_298 & & \\
\hline 561 & 1483202 & G & A & SNP & nonsynonymous & HPG27_298 & & \\
\hline$S 61$ & 1483269 & A & G & SNP & nonsynonymous & HPG27_298 & & \\
\hline 561 & 1640915 & CGAGAGAGAGAGAGAG & CGAGAGAGAGAGAGAGAG & indel & frameshift insertion & & & \\
\hline 561 & 1669898 & $\mathrm{c}$ & $T$ & SNP & synonymous & HP1450 & & \\
\hline 562 & 658 & c & $\mathrm{T}$ & SNP & synonymous & HP1529 & & \\
\hline $\mathrm{s} 62$ & 214663 & CGAGAGAGAGAGAGAG & CGAGAGAGAGAGAGAGAG & indel & intergenic & & HPG27_8 & \\
\hline 562 & 232964 & c & A & SNP & intergenic & & HPPC_00115 & \\
\hline 562 & 249555 & G & A & SNP & nonsynonymous & HP0044 & & \\
\hline 562 & 315327 & ACCCCCCCCCCC & AсCСCCCCCCCCC & indel & intergenic & & HP0103 & \\
\hline
\end{tabular}


bioRxiv preprint doi: https://doi.org/10.1101/2020.02.15.950279; this version posted February 21, 2020. The copyright holder for this

preprint (which was not certified by peer review) is the author/funder. All rights reserved. No reuse allowed without permission.

\begin{tabular}{|c|c|c|c|c|c|c|c|}
\hline 564 & 1482879 & A & $G$ & SNP & nonsynonymous & HPG27_298 & \\
\hline 564 & 1483092 & G & A & SNP & nonsynonymous & HPG27_298 & \\
\hline 564 & 1483269 & A & $G$ & SNP & nonsynonymous & HPG27_298 & \\
\hline 564 & 1484872 & $T$ & c & SNP & synonymous & HPG27_298 & \\
\hline 564 & 1484881 & $G$ & A & SNP & synonymous & HPG27_298 & \\
\hline 564 & 1484897 & c & A & SNP & nonsynonymous & HPG27_298 & \\
\hline 564 & 1484899 & c & A & SNP & synonymous & HPG27_298 & \\
\hline 564 & 1669898 & c & $T$ & SNP & synonymous & HP1450 & \\
\hline 565 & 232964 & c & A & SNP & intergenic & & HPPC_00115 \\
\hline 565 & 315327 & ACCCCCCCCCC & ACCCCCCCCCCCC & indel & intergenic & & HP0103 \\
\hline 565 & 332580 & $\mathrm{~T}$ & A & SNP & synonymous & HP0119 & \\
\hline 565 & 332598 & c & $\mathrm{T}$ & SNP & synonymous & HP0119 & \\
\hline 565 & 332632 & G & A & SNP & nonsynonymous & HP0119 & \\
\hline 565 & 332653 & c & $T$ & SNP & nonsynonymous & HP0119 & \\
\hline 565 & 332689 & $G$ & A & SNP & nonsynonymous & HP0119 & \\
\hline 565 & 374756 & CGGGGGGGGGGGG & cGGGGGGGGGGGGGG & indel & frameshift insertion & jhp0151 & \\
\hline 565 & 701037 & TGGGGGGGG & TGGGGGGG & indel & frameshift deletion & HP0499 & \\
\hline 565 & 734196 & G & A & SNP & synonymous & HPP12_0534 & \\
\hline 565 & 734198 & A & G & SNP & nonsynonymous & HPP12_0534 & \\
\hline 565 & 734200 & c & A & SNP & nonsynonymous & HPP12_0534 & \\
\hline 565 & 734220 & c & $T$ & SNP & synonymous & HPP12_0534 & \\
\hline 565 & 734234 & A & G & SNP & nonsynonymous & HPP12_0534 & \\
\hline 565 & 734241 & $\mathrm{~T}$ & A & SNP & synonymous & HPP12_0534 & \\
\hline 565 & 734256 & c & $\mathrm{T}$ & SNP & synonymous & HPP12_0534 & \\
\hline 565 & 734260 & G & A & SNP & nonsynonymous & HPP12_0534 & \\
\hline$\$ 65$ & 734271 & G & A & SNP & synonymous & HPP12_0534 & \\
\hline 565 & 734277 & c & $T$ & SNP & synonymous & HPP12_0534 & \\
\hline 565 & 734283 & c & $\mathrm{T}$ & SNP & synonymous & HPP12_0534 & \\
\hline 565 & 734286 & $\mathrm{~T}$ & $\mathrm{c}$ & SNP & synonymous & HPP12_0534 & \\
\hline 565 & 734309 & A & c & SNP & nonsynonymous & HPP12_0534 & \\
\hline 565 & 734313 & G & A & SNP & synonymous & HPP12_0534 & \\
\hline 565 & 734317 & A & c & SNP & nonsynonymous & HPP12_0534 & \\
\hline 565 & 735228 & A & $T$ & SNP & synonymous & HPP12_0534 & \\
\hline 565 & 735324 & C & G & SNP & synonymous & HPP12_0534 & \\
\hline 565 & 735333 & A & G & SNP & synonymous & HPP12_0534 & \\
\hline 565 & 735336 & G & A & SNP & synonymous & HPP12_0534 & \\
\hline 565 & 735343 & c & $T$ & SNP & synonymous & HPP12_0534 & \\
\hline$\$ 65$ & 735356 & $T$ & c & SNP & nonsynonymous & HPP12_0534 & \\
\hline 565 & 735363 & $G$ & A & SNP & synonymous & HPP12_0534 & \\
\hline 565 & 801836 & GAAAAAAAAAAAA & GAAAAAAAAAAAAA & indel & intergenic & & jhp0540 \\
\hline 565 & 805851 & A & $\mathrm{T}$ & SNP & intergenic & & HPG27_556 \\
\hline 565 & 839999 & $\mathrm{~T}$ & c & SNP & nonsynonymous & HPB8_818 & \\
\hline 565 & 958702 & CAAAAAAAAAAAAAAAA & CAAAAAAAAAAAAAAAAAA & indel & intergenic & & HPG27_680 \\
\hline 565 & 1043704 & GTTTTTTTTTTTTTT & GTTTTTTTTTTTTTTTTT & indel & frameshift insertion/intergenic & HPnc4170 & HPnc4160 \\
\hline 565 & 1122208 & $\mathrm{AG}$ & A & SNP & intergenic & & HP0895 \\
\hline S65 & 1122232 & A & AG & indel & intergenic & & HP0895 \\
\hline 565 & 1187376 & АТTTTTTT & АТTTTTTTT & indel & intergenic & & HP0947 \\
\hline 565 & 1198606 & A & c & SNP & nonsynonymous & HP0961 & \\
\hline 565 & 1284699 & AGGGGGGGGGG & AGGGGGGGGG & indel & frameshift deletion & HPG27_1018 & \\
\hline 565 & 1306585 & c & $T$ & SNP & nonsynonymous & HP0360 & \\
\hline 565 & 1318527 & $\mathrm{c}$ & A & SNP & intergenic & jhp1031 & \\
\hline 565 & 1483720 & G & $\mathrm{A}$ & SNP & synonymous & HPG27_298 & \\
\hline 565 & 1484872 & $\mathrm{~T}$ & $\mathrm{c}$ & SNP & synonymous & HPG27_298 & \\
\hline 565 & 1484881 & G & A & SNP & synonymous & HPG27_298 & \\
\hline 565 & 1484897 & c & A & SNP & nonsynonymous & HPG27_298 & \\
\hline 565 & 1484899 & c & A & SNP & synonymous & HPG27_298 & \\
\hline 565 & 1640915 & CGAGAGAGAGAGAGAG & CGAGAGAGAGAGAGAGAG & indel & frameshift insertion & & \\
\hline $\mathrm{s} 65$ & 1663529 & GTTTTT & GTTTT & indel & 1 bp deletion & & \\
\hline 566 & 143981 & TGA & $\mathrm{T}$ & indel & intergenic & & HP1400 \\
\hline 566 & 193201 & G & A & SNP & synonymous & jhp1480 & \\
\hline 566 & 332580 & $T$ & A & SNP & synonymous & HP0119 & \\
\hline 566 & 332598 & c & $T$ & SNP & synonymous & HP0119 & \\
\hline 566 & 332632 & $G$ & A & SNP & nonsynonymous & HP0119 & \\
\hline 566 & 332653 & c & $T$ & SNP & nonsynonymous & HP0119 & \\
\hline 566 & 332689 & G & A & SNP & nonsynonymous & HP0119 & \\
\hline 566 & 374767 & GGT & GGGG,GGGTG & indel & frameshift insertion & jhp0151 & \\
\hline 566 & 455599 & TGGGGGGGGGG & TGGGGGGGGGGG & indel & frameshift insertion & HP0217 & \\
\hline 566 & 546511 & CAAAAAAAAAAAA & A & indel & intergenic & & HP1243/HPG27_298 \\
\hline 566 & 701037 & TGGGGGGGG & TGGGGGGG & indel & frameshift deletion & HP0499 & \\
\hline 566 & 734196 & G & A & SNP & synonymous & HPP12_0534 & \\
\hline 566 & 734198 & A & G & SNP & nonsynonymous & HPP12_0534 & \\
\hline 566 & 734200 & c & A & SNP & nonsynonymous & HPP12_0534 & \\
\hline 566 & 734220 & c & $T$ & SNP & synonymous & HPP12_0534 & \\
\hline 566 & 734234 & A & G & SNP & nonsynonymous & HPP12_0534 & \\
\hline 566 & 734241 & $T$ & A & SNP & synonymous & HPP12_0534 & \\
\hline 566 & 734256 & c & $\mathrm{T}$ & SNP & synonymous & HPP12_0534 & \\
\hline 566 & 734260 & G & A & SNP & nonsynonymous & HPP12_0534 & \\
\hline 566 & 734271 & $G$ & A & SNP & synonymous & HPP12_0534 & \\
\hline 566 & 734277 & c & $T$ & SNP & synonymous & HPP12_0534 & \\
\hline 566 & 734283 & c & $T$ & SNP & synonymous & HPP12_0534 & \\
\hline 566 & 734286 & $T$ & $\mathrm{c}$ & SNP & synonymous & HPP12_0534 & \\
\hline S66 & 734309 & A & c & SNP & nonsynonymous & HPP12_0534 & \\
\hline S66 & 734317 & A & c & SNP & nonsynonymous & HPP12_0534 & \\
\hline 566 & 735228 & A & $T$ & SNP & synonymous & HPP12_0534 & \\
\hline 566 & 735324 & c & G & SNP & synonymous & HPP12_0534 & \\
\hline 566 & 735333 & A & G & SNP & synonymous & HPP12_0534 & \\
\hline$S 66$ & 735336 & G & A & SNP & synonymous & HPP12_0534 & \\
\hline
\end{tabular}


bioRxiv preprint doi: https://doi.org/10.1101/2020.02.15.950279; this version posted February 21, 2020. The copyright holder for this

preprint (which was not certified by peer review) is the author/funder. All rights reserved. No reuse allowed without permission.

\begin{tabular}{|c|c|c|c|c|c|c|c|c|}
\hline 566 & 735343 & c & $T$ & SNP & synonymous & HPP12_0534 & & \\
\hline 566 & 735356 & $\mathrm{~T}$ & c & SNP & nonsynonymous & HPP12_0534 & & \\
\hline 566 & 735363 & $G$ & A & SNP & synonymous & HPP12_0534 & & \\
\hline 566 & 805851 & A & $T$ & SNP & intergenic & & HPG27_556 & \\
\hline 566 & 1122208 & AG & A & SNP & intergenic & & HP0895 & \\
\hline 566 & 1122235 & G & A & SNP & intergenic & & HP0895 & \\
\hline 566 & 1187376 & АТTTTTTT & ATTTTTTTT & indel & intergenic & & HP0947 & \\
\hline 566 & 1198606 & A & c & SNP & nonsynonymous & HP0961 & & \\
\hline 566 & 1284699 & AGGGGGGGGGG & AGGGGGGGGG & indel & frameshift deletion & HPG27_1018 & & \\
\hline 566 & 1306585 & c & $\mathrm{T}$ & SNP & nonsynonymous & HP0360 & & \\
\hline 566 & 1319853 & c & A & SNP & intergenic & & HELPY_1075 & jhp1032 \\
\hline 566 & 1483720 & G & A & SNP & synonymous & HPG27_298 & & \\
\hline 566 & 1484872 & $\mathrm{~T}$ & c & SNP & synonymous & HPG27_298 & & \\
\hline 566 & 1484881 & G & A & SNP & synonymous & HPG27_298 & & \\
\hline 566 & 1484897 & $\mathrm{c}$ & A & SNP & nonsynonymous & HPG27_298 & & \\
\hline 566 & 1484899 & c & A & SNP & synonymous & HPG27_298 & & \\
\hline 566 & 1592801 & $\mathrm{~T}$ & G & SNP & synonymous & HP1354 & & \\
\hline 566 & 1640915 & CGAGAGAGAGAGAGAG & CGAGAGAGAGAGAGAGAG & indel & frameshift insertion & & & \\
\hline 567 & 92562 & $\mathrm{~T}$ & c & SNP & intergenic & & HP0993 & \\
\hline 567 & 232964 & c & $\mathrm{A}$ & SNP & intergenic & & HPPC_00115 & \\
\hline 567 & 315327 & ACCCCCCCCCC & ACCCCCCCCCCC & indel & intergenic & & HP0103 & \\
\hline 567 & 332580 & $\mathrm{~T}$ & $\mathrm{~A}$ & SNP & synonymous & HP0119 & & \\
\hline 567 & 332598 & $\mathrm{c}$ & $\mathrm{T}$ & SNP & synonymous & HP0119 & & \\
\hline 567 & 332632 & G & A & SNP & nonsynonymous & HP0119 & & \\
\hline 567 & 332653 & c & $\mathrm{T}$ & SNP & nonsynonymous & HP0119 & & \\
\hline 567 & 332689 & G & $\mathrm{A}$ & SNP & nonsynonymous & HP0119 & & \\
\hline 567 & 455599 & TGGGGGGGGGG & TGGGGGGGGGGG & indel & frameshift insertion & HP0217 & 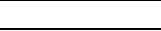 & \\
\hline 567 & 546511 & CAAAAAAAAAAAA & CAAAAAAAAAAAAA & indel & intergenic & & HP1243/HPG27_298 & \\
\hline 567 & 548865 & c & $\mathrm{T}$ & SNP & intergenic & & jhp1163 & HPG27_298 \\
\hline 567 & 548868 & $\mathrm{~T}$ & c & SNP & intergenic & & jhp1163 & HPG27_298 \\
\hline 567 & 548965 & $\mathrm{c}$ & $\mathrm{T}$ & SNP & intergenic & & jhp1163 & HPG27_298 \\
\hline 567 & 548978 & $G$ & $T$ & SNP & intergenic & & jhp1163 & HPG27_298 \\
\hline 567 & 701037 & TGGGGGGGG & TGGGGGGG & indel & frameshift deletion & HP0499 & & \\
\hline 567 & 734196 & G & A & SNP & synonymous & HPP12_0534 & & \\
\hline 567 & 734198 & A & G & SNP & nonsynonymous & HPP12_0534 & & \\
\hline s67 & 734200 & c & A & SNP & nonsynonymous & HPP12_0534 & & \\
\hline 567 & 734220 & $\mathrm{c}$ & $\mathrm{T}$ & SNP & synonymous & HPP12_0534 & & \\
\hline 567 & 734234 & A & $\mathrm{G}$ & SNP & nonsynonymous & HPP12_0534 & & \\
\hline 567 & 734241 & $T$ & A & SNP & synonymous & HPP12_0534 & & \\
\hline 567 & 734256 & c & $\mathrm{T}$ & SNP & synonymous & HPP12_0534 & & \\
\hline 567 & 734260 & G & $\mathrm{A}$ & SNP & nonsynonymous & HPP12_0534 & & \\
\hline 567 & 734271 & G & A & SNP & synonymous & HPP12_0534 & & \\
\hline 567 & 734277 & c & $\mathrm{T}$ & SNP & synonymous & HPP12_0534 & & \\
\hline 567 & 734283 & c & $T$ & SNP & synonymous & HPP12_0534 & & \\
\hline 567 & 734286 & $T$ & c & SNP & synonymous & HPP12_0534 & & \\
\hline 567 & 734309 & A & c & SNP & nonsynonymous & HPP12_0534 & & \\
\hline 567 & 734317 & A & c & SNP & nonsynonymous & HPP12_0534 & & \\
\hline 567 & 734661 & c & G & SNP & synonymous & HPP12_0534 & & \\
\hline 567 & 734733 & c & $\mathrm{T}$ & SNP & synonymous & HPP12_0534 & & \\
\hline 567 & 735228 & A & $T$ & SNP & synonymous & HPP12_0534 & & \\
\hline 567 & 735239 & c & $\mathrm{A}$ & SNP & nonsynonymous & HPP12_0534 & & \\
\hline 567 & 735324 & c & $G$ & SNP & synonymous & HPP12_0534 & & \\
\hline 567 & 735333 & A & G & SNP & synonymous & HPP12_0534 & & \\
\hline 567 & 735336 & G & A & SNP & synonymous & HPP12_0534 & & \\
\hline 567 & 735343 & $\mathrm{c}$ & $\mathrm{T}$ & SNP & synonymous & HPP12_0534 & & \\
\hline 567 & 735356 & $T$ & c & SNP & nonsynonymous & HPP12_0534 & & \\
\hline 567 & 735363 & G & A & SNP & synonymous & HPP12_0534 & & \\
\hline 567 & 801836 & GAAAAAAAAAAAA & GAAAAAAAAAAAAA & indel & intergenic & & jhp0540 & \\
\hline 567 & 839999 & $\mathrm{~T}$ & c & SNP & nonsynonymous & HPB8_818 & & \\
\hline 567 & 952086 & ТтСТСТСТСТСТСТСТС & ТТСТСТСТсTСТСТС & indel & intergenic & & HPG27_677 & \\
\hline 567 & 1043704 & GтTTTTTTTTTTTTT & GтTTTTTTTTTTTTTTTT & indel & frameshift insertion/intergenic & HPnc4170 & HP0811 & HPnc4160 \\
\hline 567 & 1122208 & $\mathrm{AG}$ & A & SNP & intergenic & & HP0895 & \\
\hline 567 & 1122232 & A & $\mathrm{AG}$ & indel & intergenic & & HP0895 & \\
\hline 567 & 1187376 & АТTTTTTT & АТTTTTTTT & indel & intergenic & & HP0947 & \\
\hline 567 & 1198606 & A & $\mathrm{c}$ & SNP & nonsynonymous & HP0961 & & \\
\hline 567 & 1284699 & AGGGGGGGGGG & AGGGGGGGGG & indel & frameshift deletion & HPG27_1018 & & \\
\hline 567 & 1306585 & c & $T$ & SNP & nonsynonymous & HP0360 & & \\
\hline 567 & 1483720 & G & A & SNP & synonymous & HPG27_298 & & \\
\hline 567 & 1484872 & $\mathrm{~T}$ & c & SNP & synonymous & HPG27_298 & & \\
\hline 567 & 1484881 & G & $\mathrm{A}$ & SNP & synonymous & HPG27_298 & & \\
\hline 567 & 1484897 & c & A & SNP & nonsynonymous & HPG27_298 & & \\
\hline 567 & 1484899 & c & A & SNP & synonymous & HPG27_298 & & \\
\hline 567 & 1640915 & CGAGAGAGAGAGAGAG & CGAGAGAGAGAGAGAGAG & indel & frameshift insertion & & & \\
\hline S68 & 232964 & c & A & SNP & intergenic & & HPPC_00115 & \\
\hline 568 & 315327 & AcccccceccC & 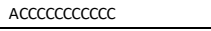 & indel & intergenic & & HP0103 & \\
\hline 568 & 332580 & $\mathrm{~T}$ & $\mathrm{~A}$ & SNP & synonymous & HP0119 & & \\
\hline 568 & 332598 & c & $T$ & SNP & synonymous & HP0119 & & \\
\hline 568 & 332632 & G & A & SNP & nonsynonymous & HP0119 & & \\
\hline 568 & 332653 & c & $T$ & SNP & nonsynonymous & HP0119 & & \\
\hline 568 & 332689 & G & A & SNP & nonsynonymous & HP0119 & & \\
\hline $\mathrm{s} 68$ & 374767 & GGT & GGGCGT & indel & frameshift insertion & jhp0151 & & \\
\hline 568 & 546511 & CAAAAAAAAAAAA & CAAAAAAAAAAAAA & indel & intergenic & & HP1243/HPG27_298 & \\
\hline 568 & 548937 & c & $T$ & SNP & intergenic & & jhp1163 & HPG27_298 \\
\hline S68 & 548965 & c & $\mathrm{T}$ & SNP & intergenic & & jhp1163 & HPG27_298 \\
\hline 568 & 548978 & G & $T$ & SNP & intergenic & & jhp1163 & HPG27_298 \\
\hline 568 & 548994 & A & $\mathrm{T}$ & SNP & intergenic & & jhp1163 & HPG27_298 \\
\hline 568 & 701037 & TGGGGGGGG & TGGGGGGG & indel & frameshift deletion & HP0499 & & \\
\hline
\end{tabular}


bioRxiv preprint doi: https://doi.org/10.1101/2020.02.15.950279; this version posted February 21, 2020. The copyright holder for this

preprint (which was not certified by peer review) is the author/funder. All rights reserved. No reuse allowed without permission.

\begin{tabular}{|c|c|c|c|c|c|c|c|c|}
\hline 568 & 734196 & $G$ & A & SNP & synonymous & HPP12_0534 & & \\
\hline 568 & 734198 & A & G & SNP & nonsynonymous & HPP12_0534 & & \\
\hline 568 & 734200 & c & A & SNP & nonsynonymous & HPP12_0534 & & \\
\hline 568 & 734220 & c & $T$ & SNP & synonymous & HPP12_0534 & & \\
\hline 568 & 734234 & A & G & SNP & nonsynonymous & HPP12_0534 & & \\
\hline 568 & 734241 & $T$ & A & SNP & synonymous & HPP12_0534 & & \\
\hline 568 & 734256 & $\mathrm{c}$ & $T$ & SNP & synonymous & HPP12_0534 & & \\
\hline 568 & 734260 & $\mathrm{G}$ & A & SNP & nonsynonymous & HPP12_0534 & & \\
\hline S68 & 734271 & $G$ & A & SNP & synonymous & HPP12_0534 & & \\
\hline 568 & 734277 & c & $T$ & SNP & synonymous & $\begin{array}{l}\text { HPP12_0534 } \\
\end{array}$ & & \\
\hline 568 & 734283 & $\mathrm{c}$ & $T$ & SNP & synonymous & HPP12_0534 & & \\
\hline S68 & 734286 & $\mathrm{~T}$ & c & SNP & synonymous & HPP12_0534 & & \\
\hline 568 & 734309 & A & $\mathrm{c}$ & SNP & nonsynonymous & HPP12_0534 & & \\
\hline 568 & 734313 & $G$ & A & SNP & synonymous & HPP12_0534 & & \\
\hline 568 & 734317 & A & $\mathrm{c}$ & SNP & nonsynonymous & HPP12_0534 & & \\
\hline 568 & 734661 & c & $G$ & SNP & synonymous & $\begin{array}{l}\text { HPP12_0534 } \\
\end{array}$ & & \\
\hline 568 & 734733 & $\mathrm{c}$ & $T$ & SNP & synonymous & HPP12_0534 & & \\
\hline 568 & 735228 & A & $T$ & SNP & synonymous & HPP12_0534 & & \\
\hline 568 & 735324 & $\mathrm{c}$ & G & SNP & synonymous & HPP12_0534 & & \\
\hline 568 & 735333 & A & $G$ & SNP & synonymous & HPP12_0534 & & \\
\hline 568 & 735336 & $G$ & A & SNP & synonymous & HPP12_0534 & & \\
\hline 568 & 735343 & c & $\mathrm{T}$ & SNP & synonymous & HPP12_0534 & & \\
\hline S68 & 735356 & $\mathrm{~T}$ & $\mathrm{c}$ & SNP & nonsynonymous & HPP12_0534 & & \\
\hline S68 & 735363 & G & A & SNP & synonymous & HPP12_0534 & & \\
\hline 568 & 801836 & GAAAAAAAAAAAA & GAAAAAAAAAAAAA & indel & intergenic & & jhp0540 & \\
\hline 568 & 839999 & $\mathrm{~T}$ & c & SNP & nonsynonymous & HPB8_818 & & \\
\hline 568 & 958702 & CAAAAAAAAAAAAAAAA & CAAAAAAAAAAAAAAAAAA & indel & intergenic & & HPG27_680 & \\
\hline 568 & 1043704 & GTTTTTTTTTTTTTT & GTTTTTTTTTTTTTTTT & indel & frameshift insertion/intergenic & HPnc4170 & HP0811 & HPnc4160 \\
\hline 568 & 1122211 & G & A & SNP & intergenic & & HP0895 & \\
\hline 568 & 1122235 & G & A & SNP & intergenic & & HP0895 & \\
\hline 568 & 1187376 & АТTTTTTT & ATTTTTTTT & indel & intergenic & & HP0947 & \\
\hline 568 & 1198606 & A & c & SNP & nonsynonymous & HP0961 & & \\
\hline 568 & 1284699 & AGGGGGGGGGG & AGGGGGGGGG & indel & frameshift deletion & HPG27_1018 & & \\
\hline 568 & 1306585 & c & $\mathrm{T}$ & SNP & nonsynonymous & HP0360 & & \\
\hline S68 & 1483720 & $\mathrm{G}$ & A & SNP & synonymous & HPG27_298 & & \\
\hline 568 & 1484872 & $\mathrm{~T}$ & c & SNP & synonymous & HPG27_298 & & \\
\hline 568 & 1484881 & $G$ & A & SNP & synonymous & HPG27_298 & & \\
\hline 568 & 1484897 & c & A & SNP & nonsynonymous & HPG27_298 & & \\
\hline 568 & 1484899 & $\mathrm{c}$ & A & SNP & synonymous & HPG27_298 & & \\
\hline 568 & 1640915 & CGAGAGAGAGAGAGAG & CGAGAGAGAGAGAGAGAG & indel & frameshift insertion & & & \\
\hline 569 & 99327 & c & $\mathrm{T}$ & SNP & synonymous & jhp0935 & & \\
\hline 569 & 155189 & GA & G & indel & frameshift deletion & jhp1438 & & \\
\hline 569 & 168379 & $\mathrm{c}$ & $T$ & SNP & synonymous & HP1547 & & \\
\hline 569 & 199597 & c & $T$ & SNP & intergenic & & HP1582 & jhp1488 \\
\hline 569 & 207029 & $T$ & $\mathrm{c}$ & SNP & nonsynonymous & HPATCC43504_00197 & & \\
\hline 569 & 214663 & CGAGAGAGAGAGAGAG & CGAGAGAGAGAGAGAGAG & indel & intergenic & & HPG27_8 & \\
\hline S69 & 276989 & G & A & SNP & nonsynonymous & HPP12_0070 & & \\
\hline 569 & 315327 & ACCCCCCCCCC & ACCCCCCCCCCCC & indel & intergenic & & HP0103 & \\
\hline 569 & 430922 & G & A & SNP & nonsynonymous & HP0194 & & \\
\hline 569 & 546511 & CAAAAAAAAAAAA & CAAAAAAAAAAAAAA & indel & intergenic & & HP1243/HPG27_298 & \\
\hline 569 & 548965 & $\mathrm{c}$ & $T$ & SNP & intergenic & & jhp1163 & HPG27_298 \\
\hline 569 & 548978 & G & $\mathrm{T}$ & SNP & intergenic & & jhp1163 & HPG27_298 \\
\hline 569 & 548986 & СтTTTTTT & CTTTTTTTT & indel & intergenic & & jhp1163 & HPG27_298 \\
\hline 569 & 680519 & $A C$ & $\mathrm{ACC}$ & indel & intergenic & & HPP12_1088 & \\
\hline 569 & 691071 & G & A & SNP & synonymous & HP0489 & & \\
\hline 569 & 691074 & A & c & SNP & synonymous & HP0489 & & \\
\hline 569 & 691077 & $G$ & A & SNP & nonsynonymous & HP0489 & & \\
\hline 569 & 691089 & A & G & SNP & synonymous & HP0489 & & \\
\hline S69 & 691091 & $\mathrm{c}$ & A & SNP & nonsynonymous & HP0489 & & \\
\hline 569 & 691101 & $T$ & $\mathrm{c}$ & SNP & synonymous & HP0489 & & \\
\hline 569 & 691113 & $\mathrm{G}$ & $\mathrm{T}$ & SNP & nonsynonymous & HP0489 & & \\
\hline 569 & 691157 & c & A & SNP & nonsynonymous & HP0489 & & \\
\hline 569 & 691173 & $\mathrm{c}$ & A & SNP & synonymous & HP0489 & & \\
\hline 569 & 691175 & A & G & SNP & nonsynonymous & HP0489 & & \\
\hline 569 & 691179 & $\mathrm{c}$ & $G$ & SNP & synonymous & HP0489 & & \\
\hline 569 & 691185 & A & G & SNP & nonsynonymous & HP0489 & & \\
\hline 569 & 691189 & c & G & SNP & nonsynonymous & HP0489 & & \\
\hline 569 & 691190 & A & c & SNP & nonsynonymous & HP0489 & & \\
\hline 569 & 801836 & GAAAAAAAAAAAA & GAAAAAAAAAAAAAA & indel & intergenic & & jhp0540 & \\
\hline 569 & 830053 & $\mathrm{c}$ & $\mathrm{T}$ & SNP & nonsynonymous & HPP12_0617 & & \\
\hline 569 & 839999 & $T$ & $\mathrm{c}$ & SNP & nonsynonymous & HPB8_818 & & \\
\hline 569 & 958702 & CAAAAAAAAAAAAAAAA & CAAAAAAAAAAAAAAAAAA & indel & intergenic & & HPG27_680 & \\
\hline 569 & 1187376 & АТTTTTTT & ATTTTTTTT & indel & intergenic & & HP0947 & \\
\hline 569 & 1192157 & $T$ & $\mathrm{c}$ & SNP & nonsynonymous & HP0953 & & \\
\hline 569 & 1332690 & c & A & SNP & synonymous & HPATCC43504_01275 & & \\
\hline 569 & 1332888 & $\mathrm{~T}$ & G & SNP & synonymous & HPATCC43504_01275 & & \\
\hline 569 & 1332893 & $T$ & $G$ & SNP & nonsynonymous & HPATCC43504_01275 & & \\
\hline 569 & 1332897 & A & G & SNP & synonymous & HPATCC43504_01275 & & \\
\hline 569 & 1332900 & G & $\mathrm{T}$ & SNP & synonymous & HPATCC43504_01275 & & \\
\hline 569 & 1332906 & $\mathrm{c}$ & $T$ & SNP & synonymous & HPATCC43504_01275 & & \\
\hline 569 & 1332954 & $\mathrm{~T}$ & G & SNP & synonymous & HPATCC43504_01275 & & \\
\hline 569 & 1332987 & $\mathrm{~T}$ & $\mathrm{c}$ & SNP & synonymous & HPATCC43504_01275 & & \\
\hline 569 & 1332996 & $T$ & $G$ & SNP & synonymous & HPATCC43504_01275 & & \\
\hline 569 & 1332997 & $G$ & $\mathrm{c}$ & SNP & nonsynonymous & HPATCC43504_01275 & & \\
\hline 569 & 1332999 & $T$ & c & SNP & synonymous & HPATCC43504_01275 & & \\
\hline 569 & 1482848 & $G$ & $T$ & SNP & nonsynonymous & HPG27_298 & & \\
\hline 569 & 1482850 & $\mathrm{~T}$ & G & SNP & nonsynonymous & HPG27_298 & & \\
\hline
\end{tabular}


bioRxiv preprint doi: https://doi.org/10.1101/2020.02.15.950279; this version posted February 21, 2020. The copyright holder for this

preprint (which was not certified by peer review) is the author/funder. All rights reserved. No reuse allowed without permission.

\begin{tabular}{|c|c|c|c|c|c|c|c|c|}
\hline 569 & 1483092 & G & A & SNP & nonsynonymous & HPG27_298 & & \\
\hline 569 & 1483202 & G & A & SNP & nonsynonymous & HPG27_298 & & \\
\hline 569 & 1483269 & A & $G$ & SNP & nonsynonymous & HPG27_298 & & \\
\hline 569 & 1592786 & GT & G & indel & frameshift deletion & HP1354 & & \\
\hline 569 & 1663529 & GTTTTT & GTTTT & indel & 1 bp deletion & & & \\
\hline 570 & 99327 & c & $T$ & SNP & synonymous & jhp0935 & & \\
\hline 570 & 168379 & c & $T$ & SNP & synonymous & HP1547 & & \\
\hline 570 & 199597 & c & $T$ & SNP & intergenic & & HP1582 & jhp1488 \\
\hline 570 & 232964 & c & A & SNP & intergenic & & HPPC_00115 & \\
\hline 570 & 276989 & G & A & SNP & nonsynonymous & HPP12_0070 & & \\
\hline 570 & 315327 & AсCCCCCCCCC & AccccccccccC & indel & intergenic & & HP0103 & \\
\hline 570 & 374756 & CGGGGGGGGGGGG & CGGGGGGGGGGGGGG & indel & frameshift insertion & jhp0151 & & \\
\hline 570 & 430922 & G & A & SNP & nonsynonymous & HP0194 & & \\
\hline 570 & 464722 & $T$ & G & SNP & synonymous & HP0227 & & \\
\hline 570 & 464740 & $\mathrm{c}$ & A & SNP & synonymous & HP0227 & & \\
\hline 570 & 464743 & A & G & SNP & synonymous & HP0227 & & \\
\hline 570 & 464764 & A & G & SNP & synonymous & HP0227 & & \\
\hline 570 & 464765 & $T$ & $\mathrm{c}$ & SNP & nonsynonymous & HP0227 & & \\
\hline 570 & 464767 & G & A & SNP & synonymous & HP0227 & & \\
\hline 570 & 464773 & G & A & SNP & synonymous & HP0227 & & \\
\hline 570 & 464779 & c & $T$ & SNP & synonymous & HP0227 & & \\
\hline 570 & 464788 & G & A & SNP & synonymous & HP0227 & & \\
\hline 570 & 464797 & A & G & SNP & synonymous & HP0227 & & \\
\hline 570 & 464800 & G & A & SNP & synonymous & HP0227 & & \\
\hline 570 & 464803 & $G$ & A & SNP & synonymous & HP0227 & & \\
\hline 570 & 546511 & CAAAAAAAAAAAA & CAAAAAAAAAAAAA & indel & intergenic & & HP1243/HPG27_298 & \\
\hline 570 & 548957 & c & $T$ & SNP & intergenic & & jhp1163 & HPG27_298 \\
\hline 570 & 548965 & c & $\mathrm{T}$ & SNP & intergenic & & jhp1163 & HPG27_298 \\
\hline 570 & 548978 & G & $\mathrm{T}$ & SNP & intergenic & & jhp1163 & HPG27_298 \\
\hline 570 & 548986 & СтTTTTTT & СТTTTTTTT & indel & intergenic & & jhp1163 & HPG27_298 \\
\hline 570 & 691071 & G & A & SNP & synonymous & HP0489 & & \\
\hline 570 & 691074 & A & c & SNP & synonymous & HP0489 & & \\
\hline 570 & 691077 & $G$ & A & SNP & nonsynonymous & HP0489 & & \\
\hline 570 & 691089 & A & G & SNP & synonymous & HP0489 & & \\
\hline 570 & 691091 & c & A & SNP & nonsynonymous & HP0489 & & \\
\hline 570 & 691101 & $\mathrm{~T}$ & c & SNP & synonymous & HP0489 & & \\
\hline 570 & 691113 & G & $T$ & SNP & nonsynonymous & HP0489 & & \\
\hline 570 & 691157 & c & A & SNP & nonsynonymous & HP0489 & & \\
\hline 570 & 691173 & c & A & SNP & synonymous & HP0489 & & \\
\hline 570 & 691175 & A & G & SNP & nonsynonymous & HP0489 & & \\
\hline 570 & 691179 & c & G & SNP & synonymous & HP0489 & & \\
\hline 570 & 691185 & A & G & SNP & nonsynonymous & HP0489 & & \\
\hline 570 & 691189 & c & G & SNP & nonsynonymous & HP0489 & & \\
\hline 570 & 691190 & A & c & SNP & nonsynonymous & HP0489 & & \\
\hline 570 & 801836 & GAAAAAAAAAAAA & GAAAAAAAAAAAAA & indel & intergenic & & jhp0540 & \\
\hline 570 & 830053 & C & $T$ & SNP & nonsynonymous & HPP12_0617 & & \\
\hline 570 & 839999 & $\mathrm{~T}$ & c & SNP & nonsynonymous & HPB8_818 & & \\
\hline 570 & 879985 & c & $\mathrm{T}$ & SNP & nonsynonymous & HP0656 & & \\
\hline s70 & 958702 & CAAAAAAAAAAAAAAAAA & CAAAAAAAAAAAAAAAAAAA & indel & intergenic & & HPG27_680 & \\
\hline 570 & 1043704 & GTTTTTTTTTTTTTT & GITTTTTTTTTTTTTTTTT & indel & frameshift insertion/intergenic & HPnc4170 & HP0811 & HPnc4160 \\
\hline 570 & 1187376 & АТTTTTTT & ATTTTTTTT & indel & intergenic & & HP0947 & \\
\hline 570 & 1192157 & $T$ & c & SNP & nonsynonymous & HP0953 & & \\
\hline 570 & 1332690 & c & A & SNP & synonymous & HPATCC43504_01275 & & \\
\hline 570 & 1332870 & c & $\mathrm{T}$ & SNP & synonymous & HPATCC43504_01275 & & \\
\hline 570 & 1332888 & $\mathrm{~T}$ & G & SNP & synonymous & HPATCC43504_01275 & & \\
\hline 570 & 1332893 & $T$ & G & SNP & nonsynonymous & HPATCC43504_01275 & & \\
\hline 570 & 1332897 & A & $G$ & SNP & synonymous & HPATCC43504_01275 & & \\
\hline 570 & 1332900 & G & $T$ & SNP & synonymous & HPATCC43504_01275 & & \\
\hline 570 & 1332906 & $\mathrm{c}$ & $\mathrm{T}$ & SNP & synonymous & HPATCC43504_01275 & & \\
\hline 570 & 1332954 & $\mathrm{~T}$ & G & SNP & synonymous & HPATCC43504_01275 & & \\
\hline 570 & 1332987 & $T$ & $\mathrm{c}$ & SNP & synonymous & HPATCC43504_01275 & & \\
\hline 570 & 1332996 & $T$ & G & SNP & synonymous & HPATCC43504_01275 & & \\
\hline 570 & 1332997 & $G$ & $\mathrm{c}$ & SNP & nonsynonymous & HPATCC43504_01275 & & \\
\hline 570 & 1332999 & $T$ & c & SNP & synonymous & HPATCC43504_01275 & & \\
\hline 570 & 1592784 & ATGTG & ATG & indel & frameshift deletion & HP1354 & & \\
\hline 570 & 1592801 & $T$ & G & SNP & synonymous & HP1354 & & \\
\hline 571 & 99327 & c & $T$ & SNP & synonymous & jhp0935 & & \\
\hline 571 & 168379 & c & $T$ & SNP & synonymous & HP1547 & & \\
\hline 571 & 199597 & c & $T$ & SNP & intergenic & & HP1582 & jhp1488 \\
\hline 571 & 276989 & G & A & SNP & nonsynonymous & HPP12_0070 & & \\
\hline S71 & 315337 & $\mathrm{c}$ & $\mathrm{CA}$ & indel & intergenic & & HP0103 & \\
\hline S71 & 430922 & G & A & SNP & nonsynonymous & HP0194 & & \\
\hline s71 & 546511 & CAAAAAAAAAAAA & CAAAAAAAAAAAAA & indel & intergenic & & HP1243/HPG27_298 & \\
\hline S71 & 548965 & c & $T$ & SNP & intergenic & & jhp1163 & HPG27_298 \\
\hline 571 & 548978 & $G$ & $T$ & SNP & intergenic & & jhp1163 & HPG27_298 \\
\hline 571 & 548986 & СТTTTTTT & СтTтTTTTT & indel & intergenic & & jhp1163 & HPG27_298 \\
\hline 571 & 691071 & G & A & SNP & synonymous & HP0489 & & \\
\hline 571 & 691074 & A & $\mathrm{c}$ & SNP & synonymous & HP0489 & & \\
\hline 571 & 691077 & G & A & SNP & nonsynonymous & HP0489 & & \\
\hline S71 & 691089 & A & G & SNP & synonymous & HP0489 & & \\
\hline$S 71$ & 691091 & c & A & SNP & nonsynonymous & HP0489 & & \\
\hline S71 & 691101 & $T$ & C & SNP & synonymous & HP0489 & & \\
\hline S71 & 691113 & G & $\mathrm{T}$ & SNP & nonsynonymous & HP0489 & & \\
\hline S71 & 691173 & c & A & SNP & synonymous & HP0489 & & \\
\hline 571 & 691175 & A & G & SNP & nonsynonymous & HP0489 & & \\
\hline S71 & 691179 & c & G & SNP & synonymous & HP0489 & & \\
\hline$S 71$ & 691185 & A & G & SNP & nonsynonymous & HP0489 & & \\
\hline
\end{tabular}




\begin{tabular}{|c|c|c|c|c|c|c|c|c|}
\hline 571 & 691189 & c & G & SNP & nonsynonymous & HP0489 & & \\
\hline S71 & 691190 & A & c & SNP & nonsynonymous & HP0489 & & \\
\hline 571 & 830053 & $\mathrm{c}$ & $T$ & SNP & nonsynonymous & HPP12_0617 & & \\
\hline 571 & 839999 & $\mathrm{~T}$ & c & SNP & nonsynonymous & HPB8_818 & & \\
\hline S71 & 879985 & $\mathrm{c}$ & $\mathrm{T}$ & SNP & nonsynonymous & HP0656 & & \\
\hline 571 & 958702 & CAAAAAAAAAAAAAAAA & CAAAAAAAAAAAAAAAAAA & indel & intergenic & & HPG27_680 & \\
\hline 571 & 1043704 & GTTTTTTTTTTTTTT & GTTTTTTTTTTTTTTTTT & indel & frameshift insertion/intergenic & HPnc4170 & HP0811 & HPnc4160 \\
\hline 571 & 1187376 & АТTTTTTT & АТTTTTTTT & indel & intergenic & & HP0947 & \\
\hline 571 & 1192157 & $T$ & $\mathrm{c}$ & SNP & nonsynonymous & HP0953 & & \\
\hline S71 & 1318502 & c & $T$ & SNP & intergenic & jhp1031 & & \\
\hline S71 & 1332690 & c & A & SNP & synonymous & HPATCC43504_01275 & & \\
\hline S71 & 1332900 & G & GT & indel & frameshift deletion & HPATCC43504_01275 & & \\
\hline S71 & 1332906 & c & $\mathrm{T}$ & SNP & synonymous & HPATCC43504_01275 & & \\
\hline S71 & 1332954 & $T$ & G & SNP & synonymous & HPATCC43504_01275 & & \\
\hline 571 & 1332987 & $T$ & $\mathrm{c}$ & SNP & synonymous & HPATCC43504_01275 & & \\
\hline 571 & 1332996 & $\mathrm{~T}$ & G & SNP & synonymous & HPATCC43504_01275 & & \\
\hline 571 & 1332997 & G & $\mathrm{c}$ & SNP & nonsynonymous & HPATCC43504_01275 & & \\
\hline S71 & 1332999 & $\mathrm{~T}$ & c & SNP & synonymous & HPATCC43504_01275 & & \\
\hline S71 & 1592135 & G & A & SNP & nonsynonymous & HP1350 & & \\
\hline 571 & 1592784 & ATGTG & ATG & indel & frameshift deletion & HP1354 & & \\
\hline 572 & 99327 & $\mathrm{c}$ & $T$ & SNP & synonymous & jhp0935 & & \\
\hline 572 & 168379 & c & $\mathrm{T}$ & SNP & synonymous & HP1547 & & \\
\hline 572 & 199597 & $\mathrm{c}$ & $\mathrm{T}$ & SNP & intergenic & & HP1582 & jhp1488 \\
\hline S72 & 276989 & G & A & SNP & nonsynonymous & HPP12_0070 & & \\
\hline 572 & 315327 & AсcсCCсCCCC & АсССсCсCCCCC & indel & intergenic & & HP0103 & \\
\hline 572 & 430922 & G & $\mathrm{A}$ & SNP & nonsynonymous & HP0194 & & \\
\hline 572 & 466390 & G & $\mathrm{A}$ & SNP & intergenic & & HP0227 & \\
\hline 572 & 466443 & G & $\mathrm{A}$ & SNP & intergenic & & HP0227 & \\
\hline 572 & 546511 & CAAAAAAAAAAAA & CAAAAAAAAAAAAA & indel & intergenic & & HP1243/HPG27_298 & \\
\hline 572 & 691071 & G & A & SNP & synonymous & HP0489 & & \\
\hline 572 & 691074 & A & c & SNP & synonymous & HP0489 & & \\
\hline 572 & 691077 & $G$ & A & SNP & nonsynonymous & HP0489 & & \\
\hline 572 & 691089 & A & G & SNP & synonymous & HP0489 & & \\
\hline 572 & 691091 & $\mathrm{c}$ & $\mathrm{A}$ & SNP & nonsynonymous & HP0489 & & \\
\hline 572 & 691101 & $\mathrm{~T}$ & c & SNP & synonymous & HP0489 & & \\
\hline S72 & 691113 & G & $\mathrm{T}$ & SNP & nonsynonymous & HP0489 & & \\
\hline 572 & 691157 & c & $\mathrm{A}$ & SNP & nonsynonymous & HP0489 & & \\
\hline 572 & 691173 & c & $\mathrm{A}$ & SNP & synonymous & HP0489 & & \\
\hline 572 & 691175 & $\mathrm{~A}$ & $G$ & SNP & nonsynonymous & HP0489 & & \\
\hline 572 & 691179 & $\mathrm{c}$ & $G$ & SNP & synonymous & HP0489 & & \\
\hline 572 & 691185 & A & G & SNP & nonsynonymous & HP0489 & & \\
\hline 572 & 691189 & c & G & SNP & nonsynonymous & HP0489 & & \\
\hline 572 & 691190 & A & c & SNP & nonsynonymous & HP0489 & & \\
\hline 572 & 801836 & GAAAAAAAAAAAA & GAAAAAAAAAAAAA & indel & intergenic & & jhp0540 & \\
\hline 572 & 830053 & $\mathrm{c}$ & $\mathrm{T}$ & SNP & nonsynonymous & HPP12_0617 & & \\
\hline 572 & 836443 & $\mathrm{c}$ & $\mathrm{T}$ & SNP & synonymous & HP0617 & & \\
\hline 572 & 839999 & $\mathrm{~T}$ & c & SNP & nonsynonymous & HPB8_818 & & \\
\hline 572 & 879985 & c & $\mathrm{T}$ & SNP & nonsynonymous & HP0656 & & \\
\hline 572 & 1043704 & GTTTTTTTTTTTTTT & GтTTTTTTTTTTTTTTTT & indel & frameshift insertion/intergenic & HPnc4170 & HP0811 & HPnc4160 \\
\hline 572 & 1187376 & АТTTTTTT & ATTTTTTTT & indel & intergenic & & HP0947 & \\
\hline 572 & 1192157 & $\mathrm{~T}$ & c & SNP & nonsynonymous & HP0953 & & \\
\hline 572 & 1332690 & $\mathrm{c}$ & $\mathrm{A}$ & SNP & synonymous & HPATCC43504_01275 & & \\
\hline 572 & 1332900 & G & $\mathrm{T}$ & SNP & synonymous & HPATCC43504_01275 & & \\
\hline 572 & 1332906 & c & $\mathrm{T}$ & SNP & synonymous & HPATCC43504_01275 & & \\
\hline 572 & 1332954 & $\mathrm{~T}$ & G & SNP & synonymous & HPATCC43504_01275 & & \\
\hline 572 & 1332987 & $T$ & c & SNP & synonymous & HPATCC43504_01275 & & \\
\hline 572 & 1332996 & $\mathrm{~T}$ & G & SNP & synonymous & HPATCC43504_01275 & & \\
\hline 572 & 1332997 & G & c & SNP & nonsynonymous & HPATCC43504_01275 & & \\
\hline 572 & 1332999 & $\mathrm{~T}$ & c & SNP & synonymous & HPATCC43504_01275 & & \\
\hline 573 & 99327 & c & $\mathrm{T}$ & SNP & synonymous & jhp0935 & & \\
\hline 573 & 168379 & c & $\mathrm{T}$ & SNP & synonymous & HP1547 & & \\
\hline 573 & 199597 & c & $T$ & SNP & intergenic & & HP1582 & jhp1488 \\
\hline 573 & 226261 & $G$ & $\mathrm{~A}$ & SNP & nonsynonymous & HP0019_1 & & \\
\hline 573 & 276989 & G & $\mathrm{A}$ & SNP & nonsynonymous & HPP12_0070 & & \\
\hline 573 & 430922 & G & $\mathrm{A}$ & SNP & nonsynonymous & HP0194 & & \\
\hline 573 & 548965 & c & $\mathrm{T}$ & SNP & intergenic & & jhp1163 & HPG27_298 \\
\hline 573 & 548978 & $G$ & $T$ & SNP & intergenic & & jhp1163 & HPG27_298 \\
\hline 573 & 548986 & СТTTTTTT & СтTTTTTTT & indel & intergenic & & jhp1163 & HPG27_298 \\
\hline 573 & 691071 & $G$ & $\mathrm{~A}$ & SNP & synonymous & HP0489 & & \\
\hline 573 & 691074 & A & $c$ & SNP & synonymous & HP0489 & & \\
\hline 573 & 691077 & G & A & SNP & nonsynonymous & HP0489 & & \\
\hline S73 & 691089 & A & G & SNP & synonymous & HP0489 & & \\
\hline 573 & 691091 & $\mathrm{c}$ & A & SNP & nonsynonymous & HP0489 & & \\
\hline 573 & 691101 & $\mathrm{~T}$ & c & SNP & synonymous & HP0489 & & \\
\hline 573 & 691113 & G & $\mathrm{T}$ & SNP & nonsynonymous & HP0489 & & \\
\hline 573 & 691173 & $\mathrm{c}$ & $\mathrm{A}$ & SNP & synonymous & HP0489 & & \\
\hline S73 & 691175 & A & G & SNP & nonsynonymous & HP0489 & & \\
\hline 573 & 691179 & c & G & SNP & synonymous & HP0489 & & \\
\hline 573 & 691185 & $A$ & $G$ & SNP & nonsynonymous & HP0489 & & \\
\hline 573 & 691189 & $\mathrm{c}$ & $G$ & SNP & nonsynonymous & HP0489 & & \\
\hline 573 & 691190 & A & $c$ & SNP & nonsynonymous & HP0489 & & \\
\hline 573 & 830053 & $\mathrm{c}$ & $\mathrm{T}$ & SNP & nonsynonymous & HPP12_0617 & & \\
\hline S73 & 839999 & $\mathrm{~T}$ & c & SNP & nonsynonymous & HPB8_818 & & \\
\hline S73 & 958719 & G & A & SNP & intergenic & & HPG27_680 & \\
\hline 573 & 984348 & $G$ & A & SNP & nonsynonymous & HP0751 & & \\
\hline 573 & 1043704 & GTTTTTTTTTTTTTT & GTTTTTTTTTTTTTTTT & indel & frameshift insertion/intergenic & HPnc4170 & HP0811 & HPnc4160 \\
\hline 573 & 1187376 & АТTTTTTT & ATTTTTTTT & indel & intergenic & & HP0947 & \\
\hline
\end{tabular}




\begin{tabular}{|c|c|c|c|c|c|c|c|c|}
\hline 573 & 1284699 & AGGGGGGGGG & AGGGGGGGGGGG & indel & frameshift deletion & HPG27_1018 & & \\
\hline 573 & 1332593 & A & c & SNP & nonsynonymous & HPATCC43504_01275 & & \\
\hline 573 & 1332595 & $T$ & A & SNP & nonsynonymous & HPATCC43504_01275 & & \\
\hline 573 & 1332690 & $\mathrm{C}$ & A & SNP & synonymous & HPATCC43504_01275 & & \\
\hline 573 & 1332906 & $\mathrm{c}$ & $T$ & SNP & synonymous & HPATCC43504_01275 & & \\
\hline 573 & 1332954 & $T$ & G & SNP & synonymous & HPATCC43504_01275 & & \\
\hline 573 & 1332987 & $T$ & c & SNP & synonymous & HPATCC43504_01275 & & \\
\hline 573 & 1332996 & $\mathrm{~T}$ & G & SNP & synonymous & HPATCC43504_01275 & & \\
\hline 573 & 1332997 & $\mathrm{G}$ & $\mathrm{c}$ & SNP & nonsynonymous & HPATCC43504_01275 & & \\
\hline 573 & 1332999 & $T$ & $\mathrm{c}$ & SNP & synonymous & HPATCC43504_01275 & & \\
\hline 573 & 1342802 & $T$ & c & SNP & nonsynonymous & jhp0433 & & \\
\hline 573 & 1461744 & $\mathrm{c}$ & A & SNP & intergenic & & HP0349 & HP0350 \\
\hline 573 & 1592784 & ATGTG & ATG & indel & frameshift deletion & HP1354 & & \\
\hline 573 & 1592801 & $\mathrm{~T}$ & G & SNP & synonymous & HP1354 & & \\
\hline 573 & 1640915 & CGAGAGAGAGAGAGAG & CGAGAGAGAGAGAGAGAG & indel & frameshift insertion & & & \\
\hline 574 & 99327 & $\mathrm{c}$ & $T$ & SNP & synonymous & jhp0935 & & \\
\hline 574 & 113930 & $T$ & A & SNP & intergenic & & HPATCC43504_00103 & \\
\hline 574 & 168379 & $\mathrm{c}$ & $T$ & SNP & synonymous & HP1547 & & \\
\hline 574 & 199597 & $\mathrm{c}$ & $\mathrm{T}$ & SNP & intergenic & & HP1582 & jhp1488 \\
\hline 574 & 214663 & CGAGAGAGAGAGAGAG & CGAGAGAGAGAGAGAGAG & indel & intergenic & & HPG27_8 & \\
\hline 574 & 226261 & $\mathrm{G}$ & A & SNP & nonsynonymous & HP0019_1 & & \\
\hline 574 & 276989 & $\mathrm{G}$ & A & SNP & nonsynonymous & HPP12_0070 & & \\
\hline 574 & 347947 & $\mathrm{G}$ & A & SNP & nonsynonymous & jhp0122 & & \\
\hline 574 & 374756 & CGGGGGGGGGGGG & CGGGGGGGGGGGGGG & indel & frameshift insertion & jhp0151 & & \\
\hline 574 & 430922 & $\mathrm{G}$ & A & SNP & nonsynonymous & HP0194 & & \\
\hline 574 & 546511 & CAAAAAAAAAAAA & A & indel & intergenic & & HP1243/HPG27_298 & \\
\hline 574 & 547618 & A & $G$ & SNP & nonsynonymous & HPG27_298 & & \\
\hline 574 & 548965 & $\mathrm{c}$ & $T$ & SNP & intergenic & & jhp1163 & HPG27_298 \\
\hline 574 & 548978 & $\mathrm{G}$ & $T$ & SNP & intergenic & & jhp1163 & HPG27_298 \\
\hline 574 & 691071 & $\mathrm{G}$ & A & SNP & synonymous & HP0489 & & \\
\hline 574 & 691074 & A & c & SNP & synonymous & HP0489 & & \\
\hline 574 & 691077 & G & A & SNP & nonsynonymous & HP0489 & & \\
\hline 574 & 691089 & A & $G$ & SNP & synonymous & HP0489 & & \\
\hline 574 & 691091 & $\mathrm{c}$ & A & SNP & nonsynonymous & HP0489 & & \\
\hline 574 & 691101 & $T$ & $\mathrm{c}$ & SNP & synonymous & HP0489 & & \\
\hline 574 & 691113 & G & $\mathrm{T}$ & SNP & nonsynonymous & HP0489 & & \\
\hline 574 & 691157 & $\mathrm{c}$ & A & SNP & nonsynonymous & HP0489 & & \\
\hline 574 & 691173 & $\mathrm{c}$ & A & SNP & synonymous & HP0489 & & \\
\hline 574 & 691175 & A & $G$ & SNP & nonsynonymous & HP0489 & & \\
\hline 574 & 691179 & $\mathrm{c}$ & $G$ & SNP & synonymous & HP0489 & & \\
\hline 574 & 691185 & A & G & SNP & nonsynonymous & HP0489 & & \\
\hline 574 & 691189 & $\mathrm{C}$ & G & SNP & nonsynonymous & HP0489 & & \\
\hline 574 & 691190 & A & c & SNP & nonsynonymous & HP0489 & & \\
\hline 574 & 734658 & c & $T$ & SNP & synonymous & HPP12_0534 & & \\
\hline 574 & 734661 & $\mathrm{C}$ & $G$ & SNP & synonymous & HPP12_0534 & & \\
\hline 574 & 734672 & $G$ & A & SNP & nonsynonymous & $\begin{array}{l}\text { HPP12_0534 } \\
\end{array}$ & & \\
\hline 574 & 734695 & A & G & SNP & nonsynonymous & HPP12_0534 & & \\
\hline 574 & 734697 & A & G & SNP & synonymous & HPP12_0534 & & \\
\hline 574 & 734703 & $T$ & $\mathrm{c}$ & SNP & synonymous & HPP12_0534 & & \\
\hline 574 & 734841 & $\begin{array}{c}\mathrm{T} \\
\mathrm{T}\end{array}$ & A & SNP & synonymous & HPP12_0534 & & \\
\hline 574 & 734842 & $\mathrm{C}$ & A & SNP & nonsynonymous & HPP12_0534 & & \\
\hline 574 & 734852 & $\mathrm{c}$ & A & SNP & nonsynonymous & HPP12_0534 & & \\
\hline 574 & 734853 & $\mathrm{~T}$ & c & SNP & synonymous & HPP12_0534 & & \\
\hline 574 & 734871 & G & A & SNP & synonymous & HPP12_0534 & & \\
\hline 574 & 734874 & $\mathrm{~T}$ & $\mathrm{c}$ & SNP & synonymous & HPP12_0534 & & \\
\hline 574 & 735072 & G & $T$ & SNP & nonsynonymous & HPP12_0534 & & \\
\hline 574 & 735126 & $T$ & c & SNP & synonymous & HPP12_0534 & & \\
\hline 574 & 735140 & A & $G$ & SNP & nonsynonymous & $\begin{array}{l}\text { HPP12_0534 } \\
\end{array}$ & & \\
\hline 574 & 735143 & $\mathrm{C}$ & $\mathrm{T}$ & SNP & nonsynonymous & HPP12_0534 & & \\
\hline 574 & 735162 & $\mathrm{~T}$ & A & SNP & synonymous & HPP12_0534 & & \\
\hline 574 & 735163 & $\mathrm{c}$ & A & SNP & nonsynonymous & HPP12_0534 & & \\
\hline 574 & 735164 & A & G & SNP & nonsynonymous & HPP12_0534 & & \\
\hline 574 & 735173 & $\mathrm{C}$ & A & SNP & nonsynonymous & HPP12_0534 & & \\
\hline 574 & 735174 & $T$ & c & SNP & synonymous & $\begin{array}{l}\text { HPP12_0534 } \\
\end{array}$ & & \\
\hline 574 & 735192 & $G$ & A & SNP & synonymous & HPP12_0534 & & \\
\hline 574 & 735195 & $T$ & c & SNP & synonymous & HPP12_0534 & & \\
\hline 574 & 801836 & GAAAAAAAAAAAA & GAAAAAAAAAAAAA & indel & intergenic & & jhp0540 & \\
\hline 574 & 805851 & A & $T$ & SNP & intergenic & & HPG27_556 & \\
\hline S74 & 830053 & $\mathrm{c}$ & $\mathrm{T}$ & SNP & nonsynonymous & HPP12_0617 & & \\
\hline 574 & 952086 & ТТСТСТСТСТСТСТСТС & TTСТСТСТСТСТСТC & indel & intergenic & & HPG27_677 & \\
\hline 574 & 1043704 & GTTTTTTTTTTTTTT & GтTTTTTTTTTTTTTTTT & indel & frameshift insertion/intergenic & HPnc4170 & HP0811 & HPnc4160 \\
\hline 574 & 1122223 & $\mathrm{c}$ & $T$ & SNP & intergenic & & HP0895 & \\
\hline 574 & 1122228 & TAAAAA & TAAAAAA & indel & intergenic & & HP0895 & \\
\hline 574 & 1187376 & ATTTTTTT & ATTTTTTTT & indel & intergenic & & HP0947 & \\
\hline 574 & 1284699 & AGGGGGGGGGG & AGGGGGGGGGG & indel & frameshift deletion & HPG27_1018 & & \\
\hline 574 & 1318527 & $\mathrm{c}$ & A & SNP & intergenic & jhp1031 & & \\
\hline 574 & 1332690 & $\mathrm{c}$ & A & SNP & synonymous & HPATCC43504_01275 & & \\
\hline 574 & 1332888 & $\mathrm{~T}$ & G & SNP & synonymous & HPATCC43504_01275 & & \\
\hline 574 & 1332893 & $T$ & G & SNP & nonsynonymous & HPATCC43504_01275 & & \\
\hline 574 & 1332897 & A & G & SNP & synonymous & HPATCC43504_01275 & & \\
\hline 574 & 1332900 & $G$ & $T$ & SNP & synonymous & HPATCC43504_01275 & & \\
\hline 574 & 1332906 & $\mathrm{c}$ & $\mathrm{T}$ & SNP & synonymous & HPATCC43504_01275 & & \\
\hline 574 & 1332954 & $T$ & $G$ & SNP & synonymous & HPATCC43504_01275 & & \\
\hline 574 & 1332987 & $T$ & c & SNP & synonymous & HPATCC43504_01275 & & \\
\hline 574 & 1332996 & $T$ & G & SNP & synonymous & HPATCC43504_01275 & & \\
\hline 574 & 1332997 & G & c & SNP & nonsynonymous & HPATCC43504_01275 & & \\
\hline 574 & 1332999 & $T$ & $\mathrm{c}$ & SNP & synonymous & HPATCC43504_01275 & & \\
\hline
\end{tabular}


bioRxiv preprint doi: https://doi.org/10.1101/2020.02.15.950279; this version posted February 21, 2020. The copyright holder for this

preprint (which was not certified by peer review) is the author/funder. All rights reserved. No reuse allowed without permission.

\begin{tabular}{|c|c|c|c|c|c|c|c|c|}
\hline 574 & 1362531 & A & $T$ & SNP & nonsynonymous & HPB8_1119 & & \\
\hline S74 & 1456630 & G & A & SNP & nonsynonymous & HP0354 & & \\
\hline 574 & 1482848 & $G$ & $T$ & SNP & nonsynonymous & HPG27_298 & & \\
\hline 574 & 1482850 & $\mathrm{~T}$ & G & SNP & nonsynonymous & HPG27_298 & & \\
\hline S74 & 1483092 & $G$ & A & SNP & nonsynonymous & HPG27_298 & & \\
\hline 574 & 1483202 & $G$ & A & SNP & nonsynonymous & HPG27_298 & & \\
\hline 574 & 1592801 & $T$ & G & SNP & synonymous & HP1354 & & \\
\hline 575 & 99327 & $\mathrm{c}$ & $\mathrm{T}$ & SNP & synonymous & jhp0935 & & \\
\hline 575 & 122766 & TTTTTTAATGAAGTT & TTT & indel & frameshift deletion & HP1003 & & \\
\hline 575 & 168379 & $\mathrm{c}$ & $\mathrm{T}$ & SNP & synonymous & HP1547 & & \\
\hline 575 & 199597 & $\mathrm{c}$ & $\mathrm{T}$ & SNP & intergenic & & HP1582 & jhp1488 \\
\hline S75 & 214663 & CGAGAGAGAGAGAGAG & CGAGAGAGAGAGAGAGAG & indel & intergenic & & HPG27_8 & \\
\hline 575 & 226261 & $G$ & A & SNP & nonsynonymous & HP0019_1 & & \\
\hline 575 & 276989 & G & A & SNP & nonsynonymous & HPP12_0070 & & \\
\hline 575 & 430922 & $G$ & A & SNP & nonsynonymous & HP0194 & & \\
\hline S75 & 546511 & CAAAAAAAAAAAA & A & indel & intergenic & & HP1243/HPG27_298 & \\
\hline 575 & 547618 & A & G & SNP & nonsynonymous & HPG27_298 & & \\
\hline 575 & 548965 & $\mathrm{c}$ & $T$ & SNP & intergenic & & jhp1163 & HPG27_298 \\
\hline 575 & 548978 & G & $T$ & SNP & intergenic & & jhp1163 & HPG27_298 \\
\hline 575 & 691071 & G & A & SNP & synonymous & HP0489 & & \\
\hline 575 & 691074 & $A$ & c & SNP & synonymous & HP0489 & & \\
\hline$S 75$ & 691077 & $G$ & A & SNP & nonsynonymous & HP0489 & & \\
\hline S75 & 691089 & A & G & SNP & synonymous & HP0489 & & \\
\hline S75 & 691091 & c & A & SNP & nonsynonymous & HP0489 & & \\
\hline 575 & 691101 & $T$ & c & SNP & synonymous & HP0489 & & \\
\hline S75 & 691113 & G & $T$ & SNP & nonsynonymous & HP0489 & & \\
\hline 575 & 691155 & $\mathrm{c}$ & $G$ & SNP & synonymous & HP0489 & & \\
\hline 575 & 691157 & $\mathrm{C}$ & A & SNP & nonsynonymous & HP0489 & & \\
\hline S75 & 691173 & $\mathrm{c}$ & A & SNP & synonymous & HP0489 & & \\
\hline 575 & 691175 & A & G & SNP & nonsynonymous & HP0489 & & \\
\hline 575 & 691179 & c & G & SNP & synonymous & HP0489 & & \\
\hline 575 & 691185 & A & G & SNP & nonsynonymous & HP0489 & & \\
\hline 575 & 691189 & c & $G$ & SNP & nonsynonymous & HP0489 & & \\
\hline 575 & 691190 & A & c & SNP & nonsynonymous & HP0489 & & \\
\hline S75 & 734658 & $\mathrm{c}$ & $\mathrm{T}$ & SNP & synonymous & HPP12_0534 & & \\
\hline S75 & 734661 & c & G & SNP & synonymous & HPP12_0534 & & \\
\hline 575 & 734672 & $G$ & A & SNP & nonsynonymous & HPP12_0534 & & \\
\hline S75 & 734695 & A & G & SNP & nonsynonymous & HPP12_0534 & & \\
\hline 575 & 734697 & $A$ & $G$ & SNP & synonymous & HPP12_0534 & & \\
\hline 575 & 734703 & $T$ & c & SNP & synonymous & HPP12_0534 & & \\
\hline 575 & 734722 & G & c & SNP & nonsynonymous & HPP12_0534 & & \\
\hline 575 & 735067 & $T$ & G & SNP & nonsynonymous & HPP12_0534 & & \\
\hline 575 & 735072 & G & $T$ & SNP & nonsynonymous & HPP12_0534 & & \\
\hline 575 & 735126 & $\mathrm{~T}$ & c & SNP & synonymous & HPP12_0534 & & \\
\hline 575 & 735140 & $A$ & $G$ & SNP & nonsynonymous & HPP12_0534 & & \\
\hline 575 & 735143 & c & $T$ & SNP & nonsynonymous & HPP12_0534 & & \\
\hline S75 & 735162 & $\mathrm{~T}$ & A & SNP & synonymous & HPP12_0534 & & \\
\hline$S 75$ & 735163 & C & A & SNP & nonsynonymous & HPP12_0534 & & \\
\hline 575 & 735164 & A & $G$ & SNP & nonsynonymous & HPP12_0534 & & \\
\hline$S 75$ & 735173 & $\mathrm{c}$ & $\mathrm{A}$ & SNP & nonsynonymous & HPP12_0534 & & \\
\hline 575 & 735174 & $T$ & c & SNP & synonymous & HPP12_0534 & & \\
\hline 575 & 735192 & G & A & SNP & synonymous & HPP12_0534 & & \\
\hline 575 & 735195 & $\mathrm{~T}$ & c & SNP & synonymous & HPP12_0534 & & \\
\hline 575 & 805851 & A & $T$ & SNP & intergenic & & HPG27_556 & \\
\hline 575 & 830053 & c & $\mathrm{T}$ & SNP & nonsynonymous & HPP12_0617 & & \\
\hline 575 & 960744 & $T$ & G & SNP & intergenic & & HP0727 & \\
\hline 575 & 1043666 & $G$ & A & SNP & intergenic & HPnc4170 & HP0811 & HPnc4160 \\
\hline 575 & 1122223 & $\mathrm{c}$ & $\mathrm{T}$ & SNP & intergenic & & HP0895 & \\
\hline 575 & 1122228 & TAAAAA & TAAAAAA & indel & intergenic & & HP0895 & \\
\hline S75 & 1124346 & CGAGAGAGAGAGAGAGAGAG & CGAGAGAGAGAGAGAGAG & indel & intergenic & & HPG27_1187 & \\
\hline 575 & 1187376 & АТTTTTTT & ATTTTTTTT & indel & intergenic & & HP0947 & \\
\hline$S 75$ & 1284699 & AGGGGGGGGGG & AGGGGGGGGGGG & indel & frameshift deletion & HPG27_1018 & & \\
\hline 575 & 1318527 & $\mathrm{c}$ & A & SNP & intergenic & jhp1031 & & \\
\hline 575 & 1332593 & A & c & SNP & nonsynonymous & HPATCC43504_01275 & & \\
\hline 575 & 1332595 & $\mathrm{~T}$ & A & SNP & nonsynonymous & HPATCC43504_01275 & & \\
\hline 575 & 1332690 & $\mathrm{c}$ & A & SNP & synonymous & HPATCC43504_01275 & & \\
\hline 575 & 1332900 & G & $\mathrm{T}$ & SNP & synonymous & HPATCC43504_01275 & & \\
\hline 575 & 1332906 & $\mathrm{c}$ & $\mathrm{T}$ & SNP & synonymous & HPATCC43504_01275 & & \\
\hline 575 & 1332954 & $T$ & $G$ & SNP & synonymous & HPATCC43504_01275 & & \\
\hline 575 & 1332987 & $\mathrm{~T}$ & c & SNP & synonymous & HPATCC43504_01275 & & \\
\hline S75 & 1332996 & $\mathrm{~T}$ & G & SNP & synonymous & HPATCC43504_01275 & & \\
\hline S75 & 1332997 & G & c & SNP & nonsynonymous & HPATCC43504_01275 & & \\
\hline 575 & 1332999 & $T$ & c & SNP & synonymous & HPATCC43504_01275 & & \\
\hline S75 & 1482848 & G & $T$ & SNP & nonsynonymous & HPG27_298 & & \\
\hline 575 & 1482850 & $T$ & $G$ & SNP & nonsynonymous & HPG27_298 & & \\
\hline 575 & 1483092 & G & A & SNP & nonsynonymous & HPG27_298 & & \\
\hline S75 & 1592784 & ATGTG & ATG & indel & frameshift deletion & HP1354 & & \\
\hline 575 & 1592801 & $\mathrm{~T}$ & G & SNP & synonymous & HP1354 & & \\
\hline 576 & 99327 & c & $T$ & SNP & synonymous & jhp0935 & & \\
\hline 576 & 119015 & A & c & SNP & nonsynonymous & HP1534 & & \\
\hline 576 & 168379 & $\mathrm{c}$ & $T$ & SNP & synonymous & HP1547 & & \\
\hline 576 & 199597 & $\mathrm{c}$ & $T$ & SNP & intergenic & & HP1582 & jhp1488 \\
\hline S76 & 226261 & G & A & SNP & nonsynonymous & HP0019_1 & & \\
\hline S76 & 276989 & G & A & SNP & nonsynonymous & HPP12_0070 & & \\
\hline 576 & 315327 & ACCCCCCCCCC & ACCCCCCCCCCC & indel & intergenic & & HP0103 & \\
\hline 576 & 413866 & A & G & SNP & synonymous & HP1535 & & \\
\hline 576 & 413887 & $\mathrm{~A}$ & G & SNP & synonymous & HP1535 & & \\
\hline
\end{tabular}




\begin{tabular}{|c|c|c|c|c|c|c|c|c|}
\hline 576 & 430922 & G & A & SNP & nonsynonymous & HP0194 & & \\
\hline 576 & 455599 & TGGGGGGGGGG & TGGGGGGGGGGG & indel & frameshift insertion & HP0217 & & \\
\hline 576 & 546511 & CAAAAAAAAAAAA & CAAAAAAAAAAAAA & indel & intergenic & & HP1243/HPG27_298 & \\
\hline 576 & 548978 & G & $\mathrm{T}$ & SNP & intergenic & & jhp1163 & HPG27_298 \\
\hline 576 & 691071 & G & A & SNP & synonymous & $\begin{array}{ll}\text { HP0489 } \\
\end{array}$ & & \\
\hline 576 & 691074 & A & c & SNP & synonymous & HP0489 & & \\
\hline 576 & 691077 & G & A & SNP & nonsynonymous & HP0489 & & \\
\hline 576 & 691089 & $\mathrm{~A}$ & $\mathrm{G}$ & SNP & synonymous & HP0489 & & \\
\hline 576 & 691091 & $\mathrm{c}$ & $\mathrm{A}$ & SNP & nonsynonymous & HP0489 & & \\
\hline 576 & 691101 & $T$ & c & SNP & synonymous & HP0489 & & \\
\hline 576 & 691113 & G & $\mathrm{T}$ & SNP & nonsynonymous & HP0489 & & \\
\hline 576 & 691173 & c & A & SNP & synonymous & HP0489 & & \\
\hline 576 & 691175 & $A$ & $G$ & SNP & nonsynonymous & HP0489 & & \\
\hline 576 & 691179 & $\mathrm{c}$ & G & SNP & synonymous & HP0489 & & \\
\hline 576 & 691185 & A & G & SNP & nonsynonymous & HP0489 & & \\
\hline 576 & 691189 & c & $G$ & SNP & nonsynonymous & $\begin{array}{ll}\text { HP0489 } \\
\end{array}$ & & \\
\hline 576 & 691190 & A & c & SNP & nonsynonymous & HP0489 & & \\
\hline 576 & 830053 & c & $\mathrm{T}$ & SNP & nonsynonymous & HPP12_0617 & & \\
\hline 576 & 839999 & $T^{\top}$ & $\mathrm{c}$ & SNP & nonsynonyymous & HPB__818 & & \\
\hline 576 & 952086 & ТтСтстстстстстстс & ТтСТстстстстстс & indel & intergenic & & HPG27_677 & \\
\hline 576 & 984348 & G & A & SNP & nonsynonymous & HP0751 & & \\
\hline 576 & 1043704 & GтTTTTTTTTTTTT & GTTTTTTTTTTTTTTTT & indel & frameshift insertion/intergenic & HPnc4170 & HP0811 & HPnc4160 \\
\hline 576 & 1187376 & АТTTTTTT & АТTTTTTTT & indel & intergenic & & HP0947 & \\
\hline 576 & 1284699 & AGGGGGGGGGG & AGGGGGGGGGGG & indel & frameshift deletion & HPG27_1018 & & \\
\hline 576 & 1318525 & $A A C$ & A & indel & frameshift deletion & jhp1031 & & \\
\hline 576 & 1332690 & $\mathrm{c}$ & $\mathrm{A}$ & SNP & synonymous & HPATCC43504_01275 & & \\
\hline 576 & 1332906 & c & $\mathrm{T}$ & SNP & synonymous & HPATCC43504_01275 & & \\
\hline 576 & 1332954 & $\mathrm{~T}$ & $G$ & SNP & synonymous & HPATCC43504_01275 & & \\
\hline 576 & 1332987 & $\mathrm{~T}$ & $\mathrm{c}$ & SNP & synonymous & HPATCC43504_01275 & & \\
\hline 576 & 1332996 & $T$ & $G$ & SNP & synonymous & HPATCC43504_01275 & & \\
\hline 576 & 1332997 & G & c & SNP & nonsynonymous & HPATCC43504_01275 & & \\
\hline 576 & 1332999 & $\mathrm{~T}$ & c & SNP & synonymous & HPATCC43504_01275 & & \\
\hline 576 & 1592801 & $\mathrm{~T}$ & $G$ & SNP & synonymous & HP1354 & & \\
\hline 576 & 1640915 & CGAGAGAGAGAGAGAG & CGAGAGAGAGAGAGAGAG & indel & frameshift insertion & & & \\
\hline 577 & 658 & $\mathrm{c}$ & $\mathrm{T}$ & SNP & synonymous & HP1529 & & \\
\hline 577 & 248956 & $c$ & $\mathrm{~T}$ & SNP & nonsynonymous & jhpo037_1 & & \\
\hline 577 & 303827 & CGGGGGGGGGGGG & CGGGGGGGGGGGGGG & indel & frameshift insertion & HPP12_0096 & & \\
\hline 577 & 315327 & AсcсссессесC & 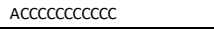 & indel & intergenic & & HP0103 & \\
\hline 577 & 374756 & CGGGGGGGGGGGG & CGGGGGGGGGGGGGG & indel & frameshift insertion & jhp0151 & & \\
\hline 577 & 412566 & $\mathrm{~T}$ & $\mathrm{c}$ & SNP & nonsynonymous & HP1534 & & \\
\hline 577 & 413983 & c & $\mathrm{T}$ & SNP & synonymous & HP1535 & & \\
\hline 577 & 431032 & c & $\mathrm{T}$ & SNP & synonymous & HP0194 & & \\
\hline 577 & 437765 & $G$ & c & SNP & intergenic & & HP0204 & HELPY_0206 \\
\hline 577 & 450628 & G & A & SNP & synonymous & HP0213 & & \\
\hline 577 & 546511 & CAAAAAAAAAAAA & CAAAAAAAAAAAAA & indel & intergenic & & HP1243/HPG27_298 & \\
\hline 577 & 548957 & $\mathrm{c}$ & $\mathrm{T}$ & SNP & intergenic & & jhp1163 & HPG27_298 \\
\hline 577 & 548965 & c & $\mathrm{T}$ & SNP & intergenic & & jhp1163 & HPG27_298 \\
\hline 577 & 548978 & G & $\mathrm{T}$ & SNP & intergenic & & jhp1163 & HPG27_298 \\
\hline 577 & 620169 & c & $\mathrm{T}$ & SNP & nonsynonymous & HPAG1_1119 & & \\
\hline 577 & 626404 & $c$ & $\mathrm{~T}$ & SNP & synonymous & HP1175 & & \\
\hline 577 & 627243 & $\mathrm{G}$ & $\mathrm{A}$ & SNP & nonsynonymous & HP1174 & & \\
\hline 577 & 701041 & G & A & SNP & nonsynonymous & HP0499 & & \\
\hline 577 & 820046 & $\mathrm{c}$ & $\mathrm{T}$ & SNP & nonsynonymous & $\begin{array}{ll}\text { HP0607 } \\
\end{array}$ & & \\
\hline 577 & 839999 & $\mathrm{~T}$ & $\mathrm{c}$ & SNP & nonsynonymous & HPB8_818 & & \\
\hline 577 & 958702 & CAAAAAAAAAAAAAAAA & CAAAAAAAAAAAAAAAAAA & indel & intergenic & & HPG27_680 & \\
\hline 577 & 1124346 & CGAGAGAGAGAGAGAGAGAG & CGAGAGAGAGAGAGAGAG & indel & intergenic & & HPG27_1187 & \\
\hline 577 & 1187376 & АTTTTTT & АТTTTTTT & indel & intergenic & & HP0947 & \\
\hline 577 & 1192612 & $\mathrm{c}$ & A & SNP & intergenic & & HP0953 & \\
\hline 577 & 1192627 & A & G & SNP & intergenic & & HP0953 & \\
\hline 577 & 1192634 & A & $G$ & SNP & intergenic & & HP0953 & \\
\hline 577 & 1192636 & G & A & SNP & intergenic & & HP0953 & \\
\hline 577 & 1192650 & $\mathrm{~T}$ & c & SNP & intergenic & & HP0953 & \\
\hline 577 & 1192686 & $\mathrm{~T}$ & G & SNP & intergenic & & HP0953 & \\
\hline 577 & 1192742 & $c$ & $\mathrm{~T}$ & SNP & intergenic & & HP0953 & \\
\hline 577 & 1192744 & $\mathrm{~T}$ & $c$ & SNP & intergenic & & HP0953 & \\
\hline 577 & 1192752 & A & $G$ & SNP & intergenic & & HP0953 & \\
\hline 577 & 1192763 & A & G & SNP & intergenic & & HP0953 & \\
\hline 577 & 1192773 & A & $G$ & SNP & intergenic & & HP0953 & \\
\hline 577 & 1192779 & $\mathrm{~T}$ & G & SNP & intergenic & & HP0953 & \\
\hline 577 & 1192784 & $\mathrm{~T}$ & $\mathrm{c}$ & SNP & intergenic & & HP0953 & \\
\hline 577 & 1192786 & c & $\mathrm{T}$ & SNP & intergenic & & HP0953 & \\
\hline 577 & 1192787 & A & $G$ & SNP & intergenic & & HР0953 & \\
\hline 577 & 1192792 & A & G & SNP & intergenic & & HP0953 & \\
\hline 577 & 1192794 & c & $\mathrm{T}$ & SNP & intergenic & & HP0953 & \\
\hline 577 & 1192820 & G & $\mathrm{A}$ & SNP & nonsynonymous & HPP12_0950 & & \\
\hline 577 & 1461824 & G & $\mathrm{T}$ & SNP & intergenic & & HP0349 & $\mathrm{HPO} 0350$ \\
\hline 577 & 1640915 & CGAGAGAGAGAGAGAG & CGAGAGAGAGAGAGAGAG & indel & frameshift insertion & & & \\
\hline 577 & 1663529 & GTTTTT & GTाT & indel & 1 bp deletion & & & \\
\hline 577 & 1669898 & $\mathrm{c}$ & $\mathrm{T}$ & SNP & synonymous & HP1450 & & \\
\hline 578 & 658 & $c$ & $\mathrm{~T}$ & SNP & synonymous & HP1529 & & \\
\hline 578 & 214663 & CGAGAGAGAGAGAGAG & CGAGAGAGAGAGAGAGAG & indel & intergenic & & HPG27_8 & \\
\hline 578 & 300860 & A & G & SNP & nonsynonymous & HP0090 & & \\
\hline 578 & 315327 & ACCCCCCCCCC & ACCCCCCCCCCC & indel & intergenic & & HP0103 & \\
\hline 578 & 431032 & $c$ & $\mathrm{~T}$ & SNP & synonymous & HP0194 & & \\
\hline 578 & 437765 & ${ }_{G}$ & $\mathrm{c}$ & SNP & intergenic & & HP0204 & HELPY_O206 \\
\hline 578 & 450628 & G & A & SNP & synonymous & HР0213 & & \\
\hline 578 & 546511 & CAAAAAAAAAAAA & CAAAAAAAAAAAAA & indel & intergenic & & HP1243/HPG27_298 & \\
\hline
\end{tabular}




\begin{tabular}{|c|c|c|c|c|c|c|c|c|}
\hline 578 & 548965 & c & $T$ & SNP & intergenic & & jhp1163 & HPG27_298 \\
\hline 578 & 548994 & A & $T$ & SNP & intergenic & & jhp1163 & HPG27_298 \\
\hline 578 & 620169 & $\mathrm{c}$ & $T$ & SNP & nonsynonymous & HPAG1_1119 & & \\
\hline 578 & 626404 & c & $\mathrm{T}$ & SNP & synonymous & HP1175 & & \\
\hline 578 & 627243 & G & A & SNP & nonsynonymous & HP1174 & & \\
\hline 578 & 701041 & G & A & SNP & nonsynonymous & HP0499 & & \\
\hline 578 & 838963 & G & $\mathrm{T}$ & SNP & nonsynonymous & jhp0562 & & \\
\hline 578 & 839999 & $T$ & c & SNP & nonsynonymous & HPB8_818 & & \\
\hline 578 & 1043704 & GTTTTTTTTTTTTTT & GTTTTTTTTTTTTTTTTT & indel & frameshift insertion/intergenic & HPnc4170 & HP0811 & HPnc4160 \\
\hline 578 & 1187376 & АТTTTTTT & АТTTTTTTT & indel & intergenic & & HP0947 & \\
\hline 578 & 1192612 & c & A & SNP & intergenic & & HP0953 & \\
\hline 578 & 1192627 & A & G & SNP & intergenic & & HP0953 & \\
\hline 578 & 1192634 & A & G & SNP & intergenic & & HP0953 & \\
\hline S78 & 1192636 & G & A & SNP & intergenic & & HP0953 & \\
\hline 578 & 1192650 & $T$ & $\mathrm{c}$ & SNP & intergenic & & HP0953 & \\
\hline 578 & 1192686 & $T$ & G & SNP & intergenic & & HP0953 & \\
\hline 578 & 1192742 & $\mathrm{c}$ & $\mathrm{T}$ & SNP & intergenic & & HP0953 & \\
\hline 578 & 1192744 & $\mathrm{~T}$ & c & SNP & intergenic & & HP0953 & \\
\hline 578 & 1192752 & A & G & SNP & intergenic & & HP0953 & \\
\hline 578 & 1192763 & A & G & SNP & intergenic & & HP0953 & \\
\hline 578 & 1192773 & A & $G$ & SNP & intergenic & & HP0953 & \\
\hline 578 & 1192779 & $T$ & G & SNP & intergenic & & HP0953 & \\
\hline 578 & 1192784 & $\mathrm{~T}$ & $\mathrm{c}$ & SNP & intergenic & & HP0953 & \\
\hline 578 & 1192786 & c & $\mathrm{T}$ & SNP & intergenic & & HP0953 & \\
\hline S78 & 1192787 & A & G & SNP & intergenic & & HP0953 & \\
\hline 578 & 1192792 & A & G & SNP & intergenic & & HP0953 & \\
\hline 578 & 1192794 & c & $T$ & SNP & intergenic & & HP0953 & \\
\hline 578 & 1192820 & G & $\mathrm{A}$ & SNP & nonsynonymous & HPP12_0950 & & \\
\hline 578 & 1230615 & A & $\mathrm{T}$ & SNP & nonsynonymous & HP0427 & & \\
\hline 578 & 1362543 & A & c & SNP & nonsynonymous & HPB8_1119 & & \\
\hline 578 & 1608139 & GT & G & indel & frameshift deletion & HP1369m & & \\
\hline 578 & 1640915 & CGAGAGAGAGAGAGAG & CGAGAGAGAGAGAGAGAG & indel & frameshift insertion & & & \\
\hline 578 & 1669898 & c & $T$ & SNP & synonymous & HP1450 & & \\
\hline S79 & 658 & c & $T$ & SNP & synonymous & HP1529 & & \\
\hline 579 & 214663 & CGAGAGAGAGAGAGAG & CGAGAGAGAGAGAGAGAG & indel & intergenic & & HPG27_8 & \\
\hline S79 & 374756 & CGGGGGGGGGGG & CGGGGGGGGGGGGGGG & indel & frameshift insertion & jhp0151 & & \\
\hline 579 & 431032 & c & $T$ & SNP & synonymous & HP0194 & & \\
\hline 579 & 437765 & G & c & SNP & intergenic & & HP0204 & HELPY_0206 \\
\hline 579 & 450628 & $G$ & A & SNP & synonymous & HP0213 & & \\
\hline 579 & 548965 & c & $T$ & SNP & intergenic & & jhp1163 & HPG27_298 \\
\hline 579 & 548978 & $\mathrm{G}$ & $\mathrm{T}$ & SNP & intergenic & & jhp1163 & HPG27_298 \\
\hline 579 & 548994 & A & $\mathrm{T}$ & SNP & intergenic & & jhp1163 & HPG27_298 \\
\hline 579 & 620169 & c & $T$ & SNP & nonsynonymous & HPAG1_1119 & & \\
\hline 579 & 626404 & c & $T$ & SNP & synonymous & HP1175 & & \\
\hline 579 & 627243 & $G$ & A & SNP & nonsynonymous & HP1174 & & \\
\hline 579 & 701041 & G & A & SNP & nonsynonymous & HP0499 & & \\
\hline 579 & 801836 & GAAAAAAAAAAAA & GAAAAAAAAAAAAA & indel & intergenic & & jhp0540 & \\
\hline 579 & 953366 & G & A & SNP & nonsynonymous & HPG27_677 & & \\
\hline 579 & 958702 & CAAAAAAAAAAAAAAAA & CAAAAAAAAAAAAAAAAAAA & indel & intergenic & & HPG27_680 & \\
\hline S79 & 1043704 & GTTTTTTTTTTTTTT & GTTTTTTTTTTTTTTTTT & indel & frameshift insertion/intergenic & HPnc4170 & HP0811 & HPnc4160 \\
\hline 579 & 1187376 & АТTTTTTT & ATTTTTTTT & indel & intergenic & & HP0947 & \\
\hline 579 & 1192612 & c & $\mathrm{A}$ & SNP & intergenic & & HP0953 & \\
\hline 579 & 1192627 & A & G & SNP & intergenic & & HP0953 & \\
\hline 579 & 1192634 & A & G & SNP & intergenic & & HP0953 & \\
\hline 579 & 1192636 & G & A & SNP & intergenic & & HP0953 & \\
\hline 579 & 1192650 & $T$ & c & SNP & intergenic & & HP0953 & \\
\hline 579 & 1192686 & $T$ & $G$ & SNP & intergenic & & HP0953 & \\
\hline S79 & 1192742 & C & $T$ & SNP & intergenic & & HP0953 & \\
\hline 579 & 1192744 & $\mathrm{~T}$ & $\mathrm{c}$ & SNP & intergenic & & HP0953 & \\
\hline 579 & 1192752 & A & G & SNP & intergenic & & HP0953 & \\
\hline 579 & 1192763 & A & G & SNP & intergenic & & HP0953 & \\
\hline 579 & 1192773 & A & G & SNP & intergenic & & HP0953 & \\
\hline 579 & 1192779 & $T$ & $G$ & SNP & intergenic & & HP0953 & \\
\hline 579 & 1192784 & $T$ & c & SNP & intergenic & & HP0953 & \\
\hline 579 & 1192786 & c & $\mathrm{T}$ & SNP & intergenic & & HP0953 & \\
\hline 579 & 1192787 & A & $G$ & SNP & intergenic & & HP0953 & \\
\hline 579 & 1192792 & A & G & SNP & intergenic & & HP0953 & \\
\hline 579 & 1192794 & c & $T$ & SNP & intergenic & & HP0953 & \\
\hline 579 & 1192820 & $G$ & A & SNP & nonsynonymous & HPP12_0950 & & \\
\hline 579 & 1295885 & $\begin{array}{l}\text { GATATATATATATATATAT } \\
\end{array}$ & GATATATATATATATAT & indel & intergenic & & HPATCC43504_01238 & \\
\hline 579 & 1362557 & G & $\mathrm{c}$ & SNP & synonymous & HPB8_1119 & & \\
\hline 579 & 1593602 & TGGGGGGGGGGG & TGGGGGGGGGGGG & indel & frameshift insertion & HP1354 & & \\
\hline 579 & 1640915 & CGAGAGAGAGAGAGAG & CGAGAGAGAGAGAGAGAG & indel & frameshift insertion & & & \\
\hline 579 & 1669898 & c & $T$ & SNP & synonymous & HP1450 & & \\
\hline 580 & 658 & c & $T$ & SNP & synonymous & HP1529 & & \\
\hline 580 & 232964 & c & $\mathrm{A}$ & SNP & intergenic & & HPPC_00115 & \\
\hline 580 & 248956 & $\mathrm{c}$ & $\mathrm{T}$ & SNP & nonsynonymous & jhp0037_1 & & \\
\hline 580 & 267811 & A & G & SNP & nonsynonymous & HP0057 & & \\
\hline 580 & 303827 & CGGGGGGGGGGGG & CGGGGGGGGGGGGGG & indel & frameshift insertion & HPP12_0096 & & \\
\hline 580 & 315327 & ACCCCCCCCCC & ACCCCCCCCCCC & indel & intergenic & & HP0103 & \\
\hline 580 & 374756 & CGGGGGGGGGGGG & CGGGGGGGGGGGGGG & indel & frameshift insertion & jhp0151 & & \\
\hline 580 & 431032 & c & $\mathrm{T}$ & SNP & synonymous & HP0194 & & \\
\hline 580 & 437765 & G & c & SNP & intergenic & & HP0204 & HELPY_0206 \\
\hline 580 & 450628 & G & A & SNP & synonymous & HP0213 & & \\
\hline 580 & 546511 & CAAAAAAAAAAAA & CAAAAAAAAAAAAA & indel & intergenic & & HP1243/HPG27_298 & \\
\hline 580 & 620169 & c & $T$ & SNP & nonsynonymous & HPAG1_1119 & & \\
\hline 580 & 626404 & C & $T$ & SNP & synonymous & HP1175 & & \\
\hline
\end{tabular}


bioRxiv preprint doi: https://doi.org/10.1101/2020.02.15.950279; this version posted February 21, 2020. The copyright holder for this preprint (which was not certified by peer review) is the author/funder. All rights reserved. No reuse allowed without permission.

\begin{tabular}{|c|c|c|c|c|c|c|c|c|}
\hline 580 & 627243 & G & A & SNP & nonsynonymous & HP1174 & & \\
\hline 580 & 701041 & G & A & SNP & nonsynonymous & HP0499 & & \\
\hline 580 & 801836 & GAAAAAAAAAAAA & GAAAAAAAAAAAAA & indel & intergenic & & jhp0540 & \\
\hline 580 & 820046 & $\mathrm{c}$ & $T$ & SNP & nonsynonymous & HP0607 & & \\
\hline 580 & 958719 & G & A & SNP & intergenic & & HPG27_680 & \\
\hline 580 & 1043704 & GTTTTTTTTTTTTTT & GTTTTTTTTTTTTTTTT & indel & frameshift insertion/intergenic & HPnc4170 & HP0811 & HPnc4160 \\
\hline 580 & 1124346 & CGAGAGAGAGAGAGAGAGAG & CGAGAGAGAGAGAGAGAG & indel & intergenic & & HPG27_1187 & \\
\hline 580 & 1187376 & АТTTTTTT & АТTTTTTTT & indel & intergenic & & HP0947 & \\
\hline 580 & 1192612 & c & A & SNP & intergenic & & HP0953 & \\
\hline 580 & 1192627 & A & G & SNP & intergenic & & HP0953 & \\
\hline 580 & 1192634 & A & G & SNP & intergenic & & HP0953 & \\
\hline 580 & 1192636 & G & A & SNP & intergenic & & HP0953 & \\
\hline 580 & 1192650 & $\mathrm{~T}$ & c & SNP & intergenic & & HP0953 & \\
\hline 580 & 1192686 & $T$ & G & SNP & intergenic & & HP0953 & \\
\hline 580 & 1192742 & c & $T$ & SNP & intergenic & & HP0953 & \\
\hline 580 & 1192744 & $T$ & c & SNP & intergenic & & HP0953 & \\
\hline 580 & 1192752 & A & G & SNP & intergenic & & HP0953 & \\
\hline 580 & 1192763 & A & G & SNP & intergenic & & HP0953 & \\
\hline 580 & 1192773 & A & G & SNP & intergenic & & HP0953 & \\
\hline 580 & 1192779 & $T$ & G & SNP & intergenic & & HP0953 & \\
\hline 580 & 1192784 & $T$ & c & SNP & intergenic & & HP0953 & \\
\hline 580 & 1192786 & c & $T$ & SNP & intergenic & & HP0953 & \\
\hline 580 & 1192787 & A & G & SNP & intergenic & & HP0953 & \\
\hline 580 & 1192792 & A & G & SNP & intergenic & & HP0953 & \\
\hline 580 & 1192794 & c & $T$ & SNP & intergenic & & HP0953 & \\
\hline 580 & 1192820 & G & A & SNP & nonsynonymous & HPP12_0950 & & \\
\hline 580 & 1640915 & CGAGAGAGAGAGAGAG & CGAGAGAGAGAGAGAGAG & indel & frameshift insertion & & & \\
\hline$\$ 80$ & 1669898 & $\mathrm{C}$ & $T$ & SNP & synonymous & HP1450 & & \\
\hline
\end{tabular}


bioRxiv preprint doi: https://doi.org/10.1101/2020.02.15.950279; this version posted February 21, 2020. The copyright holder for this

preprint (which was not certified by peer review) is the author/funder. All rights reserved. No reuse allowed without permission.

Supplementary Information 3 | Summary of mutations in the isolates recovered from $\mathrm{H}$. pylori-infected C57BL/6 mice.

Number of mutations in the isolates of 10 strains recovered from $H$. pylori-infected mice stomachs 8 weeks after post infection were listed.

\begin{tabular}{|c|c|c|c|c|c|c|c|c|c|c|c|}
\hline \multirow{2}{*}{$\begin{array}{c}\text { Animal } \\
\text { No. }\end{array}$} & \multirow{2}{*}{$\begin{array}{l}\text { Strain } \\
\text { Name }\end{array}$} & \multirow{2}{*}{$\begin{array}{l}\text { Total No. } \\
\text { of } \\
\text { mutations }\end{array}$} & \multicolumn{4}{|c|}{ SNPs (single nucleotide polymorphysms) } & \multicolumn{3}{|c|}{ indel (insertion/deletion) } & \multirow{2}{*}{$\begin{array}{c}\text { SNPs rate } \\
\text { per base } \\
\text { per year }\end{array}$} & \multirow{2}{*}{$\begin{array}{l}\text { indel rate } \\
\text { per base } \\
\text { per year }\end{array}$} \\
\hline & & & total & $\begin{array}{c}\text { nonsynon } \\
\text { ymous }\end{array}$ & $\begin{array}{c}\text { synonymo } \\
\text { us }\end{array}$ & intergenic & Total & genic & intergenic & & \\
\hline \multirow{2}{*}{ \#1 } & Hp1 & 37 & 13 & 5 & 7 & 1 & 24 & 8 & 16 & $5.04 \mathrm{E}-05$ & $9.31 \mathrm{E}-05$ \\
\hline & $\mathrm{Hp} 2$ & 36 & 16 & 5 & 9 & 2 & 20 & 7 & 13 & $6.20 \mathrm{E}-05$ & $7.76 \mathrm{E}-05$ \\
\hline \multirow{2}{*}{ \#2 } & Hp3 & 38 & 16 & 6 & 8 & 2 & 22 & 8 & 14 & $6.20 \mathrm{E}-05$ & 8.53E-05 \\
\hline & $\mathrm{Hp} 4$ & 38 & 18 & 6 & 9 & 3 & 20 & 7 & 13 & $6.98 \mathrm{E}-05$ & 7.76E-05 \\
\hline \multirow{2}{*}{ \#3 } & $\mathrm{Hp} 5$ & 34 & 15 & 6 & 7 & 2 & 19 & 6 & 13 & 5.82E-05 & 7.37E-05 \\
\hline & Hp6 & 33 & 10 & 4 & 5 & 1 & 23 & 7 & 16 & $3.88 \mathrm{E}-05$ & $8.92 \mathrm{E}-05$ \\
\hline \multirow{2}{*}{ \#4 } & $\mathrm{Hp} 7$ & 45 & 25 & 11 & 12 & 2 & 20 & 10 & 10 & 9.69E-05 & 7.76E-05 \\
\hline & Hр8 & 42 & 19 & 7 & 10 & 2 & 23 & 8 & 15 & 7.37E-05 & 8.92E-05 \\
\hline \multirow{4}{*}{ \#5 } & $\mathrm{Hp9}$ & 46 & 30 & 11 & 17 & 2 & 16 & 5 & 11 & 1.16E-04 & $6.20 \mathrm{E}-05$ \\
\hline & Hp10 & 36 & 13 & 5 & 7 & 1 & 23 & 8 & 15 & 5.04E-05 & $8.92 \mathrm{E}-05$ \\
\hline & & & & & & & & & Average & $6.79 \mathrm{E}-05$ & 8.14E-05 \\
\hline & & & & & & & & & SD & $2.32 \mathrm{E}-05$ & $9.50 \mathrm{E}-06$ \\
\hline
\end{tabular}


bioRxiv preprint doi: https://doi.org/10.1101/2020.02.15.950279; this version posted February 21, 2020. The copyright holder for this

preprint (which was not certified by peer review) is the author/funder. All rights reserved. No reuse allowed without permission.

\begin{tabular}{|c|c|c|c|c|c|c|c|c|}
\hline $\begin{array}{l}\text { Strain } \\
\text { name }\end{array}$ & $\begin{array}{l}\text { Position in } \\
\text { ATCC43504 }\end{array}$ & ATCC43504 & Reisolate & & Type of difference & Annotation & $\begin{array}{l}\text { Gene nearby the } \\
\text { intergenic region (\#1) }\end{array}$ & $\begin{array}{l}\text { Gene nearby the } \\
\text { intergenic region (\#2) }\end{array}$ \\
\hline Hp1 & 658 & $\mathrm{c}$ & $\mathrm{T}$ & SNP & synonymous & HP1529 & & \\
\hline $\mathrm{Hp} 1$ & 146525 & CAAAAAAAAAAAA & CAAAAAAAAAAAAAAA & indel & intergenic & & HP1397 & \\
\hline $\mathrm{Hp} 1$ & 214663 & CGAGAGAGAGAGAGA & CGAGAGAGAGAGAGAGA & indel & frameshift insertion & HPG27_8 & & \\
\hline $\mathrm{Hp} 1$ & 249268 & c & $T$ & SNP & nonsynonymous & HPB8_1582 & & \\
\hline Hp1 & 303827 & CGGGGGGGGGGGG & CGGGGGGGGGGGGGG & indel & frameshift insertion & HPP12_0096 & & \\
\hline Hp1 & 315327 & AсcссессесCC & AссссссессесC & indel & intergenic & & HP0103 & \\
\hline Hp1 & 431032 & $\mathrm{c}$ & $\mathrm{T}$ & SNP & synonymous & HP0194 & & \\
\hline $\mathrm{Hp} 1$ & 437765 & $G$ & c & SNP & intergenic & & HP0204 & HELPY_0206 \\
\hline Hp1 & 450628 & $G$ & A & SNP & synonymous & HP0213 & & \\
\hline $\mathrm{Hp1}$ & 455599 & TGGGGGGGGGG & TGGGGGGGGGGG & indel & frameshift insertion & HP0217 & & \\
\hline Hp1 & 483804 & $\mathrm{~T}$ & TTAAATACA & indel & intergenic & & HPATCC43504_00472 & \\
\hline Hp1 & 546124 & TATTTAATCTT & $\mathrm{T}$ & indel & intergenic & & HP1243/HPG27_298 & \\
\hline Hp1 & 546511 & CAAAAAAAAAAAA & CAAAAAAAAAAAAA & indel & intergenic & & HP1243/HPG27_298 & \\
\hline $\mathrm{Hp} 1$ & 581837 & ATCAAATACTCAAATAC & A & indel & intergenic & & HPATCC43504_00587 & \\
\hline $\mathrm{Hp} 1$ & 620169 & $\mathrm{c}$ & $T$ & SNP & nonsynonymous & HP1180 & & \\
\hline $\mathrm{Hp} 1$ & 626404 & $\mathrm{c}$ & $T$ & SNP & synonymous & HP1175 & & \\
\hline Hp1 & 627243 & G & A & SNP & nonsynonymous & HP1174 & & \\
\hline Hp1 & 649248 & TG & TGG & indel & frameshift insertion & HP1156 & & \\
\hline $\mathrm{Hp} 1$ & 701041 & $G$ & A & SNP & nonsynonymous & HP0499 & & \\
\hline Hp1 & 801836 & GAAAAAAAAAAAA & GAAAAAAAAAAAAAA & indel & intergenic & & jhp0540 & \\
\hline Hp1 & 840225 & GCAAAATACGATGATCTCACCA & G & indel & nonframeshift deletion & jhp0563 & & \\
\hline Hp1 & 945518 & c & $T$ & SNP & synonymous & jhp0853 & & \\
\hline Hp1 & 958574 & CGAGAGAGAGAGA & CGAGAGAGAGAGAGA & indel & intergenic & & HPG27_680 & \\
\hline Hp1 & 958702 & CAAAAAAAAAAAAAAAA & CAAAAGAAAAAAAAAAAAAA & indel & intergenic & & HPG27_680 & \\
\hline $\mathrm{Hp} 1$ & 1043704 & GTTTTTTTTTTTTTT & GтTTTTTTTTTTTTTTTT & indel & frameshift insertion/intergenic & HPnc4170 & HP0811 & HPnc4160 \\
\hline $\mathrm{Hp1}$ & 1102157 & GTTTTTTTTTTTT & GTTTTTTTTTTT & indel & intergenic & & HP0876 & HP0875 \\
\hline Hp1 & 1124346 & CGAGAGAGAGAGAGAGAGA & CGAGAGAGAGAGAGAGA & indel & intergenic & & HPG27_1187 & \\
\hline Hp1 & 1124501 & GTTTTTTTTTTTT & GTTTTTTTTTT & indel & intergenic & & HPG27_1187 & \\
\hline Hp1 & 1187376 & АТTTTTTT & АТTTTTTTT & indel & intergenic & & HP0947 & \\
\hline Hp1 & 1230599 & TTCAAGCAA & $\mathrm{T}$ & indel & frameshift deletion & HP0427 & & \\
\hline $\mathrm{Hp} 1$ & 1319839 & CAAAAAAAAAAAAA & CCAAAAAAAAAAAAAAA & indel & intergenic & & HELPY_1075 & jhp1032 \\
\hline $\mathrm{Hp1}$ & 1331327 & $G$ & $T$ & SNP & nonsynonymous & HPATCC43504_01275 & & \\
\hline Hp1 & 1331328 & G & A & SNP & synonymous & HPATCC43504_01275 & & \\
\hline Hp1 & 1592784 & ATG & A & indel & frameshift deletion & HP1354 & & \\
\hline Hp1 & 1593602 & TGGGGGGGGGGG & TGGGGGGGGGGGG & indel & frameshift insertion & HP1354 & & \\
\hline $\mathrm{Hp} 1$ & 1627016 & TGGGGGGGGGGGGG & TGGGGGGGGGGGGGGGG & indel & intergenic & & HP1406 & HELPY_1371 \\
\hline $\mathrm{Hp} 1$ & 1669898 & $\mathrm{c}$ & $T$ & SNP & synonymous & HP1450 & & \\
\hline $\mathrm{Hp2}$ & 658 & c & $\mathrm{T}$ & SNP & synonymous & HP1529 & & \\
\hline $\mathrm{Hp} 2$ & 146525 & CAAAAAAAAAAAA & CAAAAAAAAAAAAAAA & indel & intergenic & & HP1397 & \\
\hline $\mathrm{Hp} 2$ & 214663 & CGAGAGAGAGAGAGA & CGAGAGAGAGAGAGAGA & indel & frameshift insertion & HPG27_8 & & \\
\hline Hp2 & 232964 & CAAAAAAAAAAAAAAAAAAAAA & CAAAAAAAAAAAAAAAAAAAAAAA & indel & intergenic & & HPPC_00115 & \\
\hline $\mathrm{Hp} 2$ & 249268 & c & $T$ & SNP & nonsynonymous & HPB8_1582 & & \\
\hline $\mathrm{Hp2}$ & 303827 & CGGGGGGGGGGGG & CGGGGGGGGGGGGGG & indel & frameshift insertion & HPP12_0096 & & \\
\hline Hp2 & 315327 & ACCCCCCCCCC & ACCCCCCCCCCC & indel & intergenic & & HP0103 & \\
\hline $\mathrm{Hp} 2$ & 431032 & $\mathrm{c}$ & $\mathrm{T}$ & SNP & synonymous & HP0194 & & \\
\hline $\mathrm{Hp} 2$ & 437765 & G & c & SNP & intergenic & & HP0204 & HELPY_0206 \\
\hline $\mathrm{Hp} 2$ & 450628 & G & A & SNP & synonymous & HP0213 & & \\
\hline $\mathrm{Hp} 2$ & 455599 & TGGGGGGGGGG & TGGGGGGGGGGG & indel & frameshift insertion & HP0217 & & \\
\hline $\mathrm{Hp2}$ & 464722 & $\mathrm{~T}$ & G & SNP & synonymous & HP0227 & & \\
\hline $\mathrm{Hp} 2$ & 464740 & $\mathrm{c}$ & A & SNP & synonymous & HP0227 & & \\
\hline $\mathrm{Hp} 2$ & 464743 & A & G & SNP & synonymous & HP0227 & & \\
\hline $\mathrm{Hp} 2$ & 546511 & CAAAAAAAAAAAA & CAAAAAAAAAAAAA & indel & intergenic & & HP1243/HPG27_298 & \\
\hline $\mathrm{Hp} 2$ & 548978 & G & $T$ & SNP & intergenic & & jhp1163 & HPG27_298 \\
\hline $\mathrm{Hp2}$ & 581837 & ATCAAATACTCAAATACTCAAATAC & A & indel & intergenic & & HPATCC43504_00587 & \\
\hline $\mathrm{Hp2}$ & 620169 & c & $\mathrm{T}$ & SNP & nonsynonymous & HP1180 & & \\
\hline $\mathrm{Hp} 2$ & 626404 & $\mathrm{c}$ & $\mathrm{T}$ & SNP & synonymous & HP1175 & & \\
\hline $\mathrm{Hp2}$ & 627243 & G & A & SNP & nonsynonymous & HP1174 & & \\
\hline $\mathrm{Hp2}$ & 649248 & TG & TGG & indel & frameshift insertion & HP1156 & & \\
\hline $\mathrm{Hp} 2$ & 701041 & G & A & SNP & nonsynonymous & HP0499 & & \\
\hline $\mathrm{Hp} 2$ & 801836 & GAAAAAAAAAAAA & GAAAAAAAAAAAAAA & indel & intergenic & & jhp0540 & \\
\hline $\mathrm{Hp2}$ & 945518 & c & $\mathrm{T}$ & SNP & synonymous & jhp0853 & & \\
\hline $\mathrm{Hp} 2$ & 958574 & CGAGAGAGAGAGA & CGAGAGAGAGAGAGA & indel & intergenic & & HPG27_680 & \\
\hline $\mathrm{Hp} 2$ & 958702 & CAAAAAAAAAAAAAAAA & CAATAAAAAAAAAAAAAAAA & indel & intergenic & & HPG27_680 & \\
\hline $\mathrm{Hp} 2$ & 1043704 & GTTTTTTTTTTTTTT & GTTTTTTTTTTTTTTTTT & indel & frameshift insertion/intergenic & HPnc4170 & HP0811 & HPnc4160 \\
\hline $\mathrm{Hp} 2$ & 1102157 & GTTTTTTTTTTTT & GTTTTTTTTTTT & indel & intergenic & & HP0876 & HP0875 \\
\hline $\mathrm{Hp} 2$ & 1124501 & GTTTTTTTTTTTT & GTTTTTTTTTT & indel & intergenic & & HPG27_1187 & \\
\hline $\mathrm{Hp} 2$ & 1187376 & АТTTTTTT & АТTTTTTTT & indel & intergenic & & HP0947 & \\
\hline $\mathrm{Hp} 2$ & 1423237 & $\mathrm{G}$ & A & SNP & nonsynonymous & HP1068 & & \\
\hline $\mathrm{Hp} 2$ & 1592784 & ATG & A & indel & frameshift deletion & HP1354 & & \\
\hline $\mathrm{Hp} 2$ & 1593602 & TGGGGGGGGGGG & TGGGGGGGGGGGG & indel & frameshift insertion & HP1354 & & \\
\hline $\mathrm{Hp} 2$ & 1627016 & TGGGGGGGGGGGGG & TGGGGGGGGGGGGGGGGG & indel & intergenic & & HP1406 & HELPY_1371 \\
\hline $\mathrm{Hp2}$ & 1640915 & CGAGAGAGAGAGAGA & CGAGAGAGAGAGAGAGA & indel & frameshift insertion & jhp1312 & & \\
\hline $\mathrm{Hp} 2$ & 1669898 & $\mathrm{c}$ & $\mathrm{T}$ & SNP & synonymous & HP1450 & & \\
\hline $\mathrm{Hp} 3$ & 658 & $\mathrm{c}$ & $\mathrm{T}$ & SNP & synonymous & HP1529 & & \\
\hline $\mathrm{Hp} 3$ & 214663 & $\begin{array}{l}\text { CGAGAGAGAGAGAGA } \\
\end{array}$ & CGAGAGAGAGAGAGAGA & indel & frameshift insertion & HPG27_8 & & \\
\hline $\mathrm{Hp} 3$ & 249555 & G & A & SNP & nonsynonymous & HP0044 & & \\
\hline Нp3 & 303827 & CGGGGGGGGGGGG & CGGGGGGGGGGGGGG & indel & frameshift insertion & HPP12_0096 & & \\
\hline $\mathrm{Hp} 3$ & 315327 & ACCCCCCCCCC & ACCCCCCCCCCC & indel & intergenic & & HP0103 & \\
\hline $\mathrm{Hp} 3$ & 413866 & $\mathrm{~A}$ & G & SNP & synonymous & HP1535 & & \\
\hline $\mathrm{Hp} 3$ & 413983 & c & $T$ & SNP & synonymous & HP1535 & & \\
\hline $\mathrm{Hp} 3$ & 431032 & c & $T$ & SNP & synonymous & HP0194 & & \\
\hline $\mathrm{Hp} 3$ & 437765 & $G$ & c & SNP & intergenic & & HP0204 & HELPY_0206 \\
\hline $\mathrm{Hp} 3$ & 450628 & G & A & SNP & synonymous & HP0213 & & \\
\hline $\mathrm{Hp} 3$ & 455599 & TGGGGGGGGGG & TGGGGGGGGGGG & indel & frameshift insertion & HP0217 & & \\
\hline Hр3 & 483804 & TTAAATACA & T & indel & intergenic & & HPATCC43 & \\
\hline
\end{tabular}


bioRxiv preprint doi: https://doi.org/10.1101/2020.02.15.950279; this version posted February 21, 2020. The copyright holder for this

preprint (which was not certified by peer review) is the author/funder. All rights reserved. No reuse allowed without permission.

\begin{tabular}{|c|c|c|c|c|c|c|c|c|}
\hline \multirow{2}{*}{$\begin{array}{c}\begin{array}{c}\text { Strain } \\
\text { name }\end{array} \\
\mathrm{Hp} 3\end{array}$} & \multirow{2}{*}{ 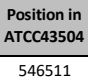 } & ATCC43504 & Reisolate & & Type of difference & Annotation & $\begin{array}{l}\text { Gene nearby the } \\
\text { intergenic region (\#1) }\end{array}$ & $\begin{array}{l}\text { Gene nearby the } \\
\text { intergenic region (\#2) }\end{array}$ \\
\hline & & CAAAAAAAAAAAA & CAAAAAAAAAAAAA & indel & intergenic & & HP1243/HPG27_298 & \\
\hline Hр3 & 548979 & TA & TTGA & indel & intergenic & & jhp1163 & HPG27_298 \\
\hline Hр3 & 581837 & ATCAAATACTCAAATACTCAAATAC & A & indel & intergenic & & HPATCC43504_00587 & \\
\hline Hр3 & 620169 & $\mathrm{c}$ & $T$ & SNP & nonsynonymous & HP1180 & & \\
\hline Hр3 & 626404 & $\mathrm{c}$ & $T$ & SNP & synonymous & HP1175 & & \\
\hline $\mathrm{Hp} 3$ & 627243 & G & A & SNP & nonsynonymous & HP1174 & & \\
\hline $\mathrm{Hp} 3$ & 701041 & $G$ & A & SNP & nonsynonymous & HP0499 & & \\
\hline $\mathrm{Hp} 3$ & 801836 & GAAAAAAAAAAAA & GAAAAAAAAAAAAAA & indel & intergenic & & jhp0540 & \\
\hline Hp3 & 958702 & CAAAAAAAAAAAAAAAA & CAAAAAAAAAAAAAAAAAAA & indel & intergenic & & HPG27_680 & \\
\hline $\mathrm{Hp} 3$ & 1043704 & GITTTTTTTTTTTTT & GTTTTTTTTTTTTTTTTT & indel & frameshift insertion/intergenic & HPnc4170 & HP0811 & HPnc4160 \\
\hline Hр3 & 1102157 & GTTTTTTTTTTTT & GTTTTTTTTTTT & indel & intergenic & & HP0876 & HP0875 \\
\hline Hр3 & 1122223 & $\mathrm{c}$ & $T$ & SNP & intergenic & & HP0895 & HPnc4510 \\
\hline $\mathrm{Hp} 3$ & 1122228 & TAAAAA & TAAAAAA & indel & intergenic & & HP0895 & HPnc4510 \\
\hline Hp3 & 1124501 & GTTTTTTTTTTTT & GTTTTTTTTTT & indel & intergenic & & HPG27_1187 & \\
\hline Hp3 & 1187376 & АТTTTTTT & АТTTTTTTT & indel & intergenic & & HP0947 & \\
\hline Hр3 & 1331327 & $G$ & $T$ & SNP & nonsynonymous & HPATCC43504_01275 & & \\
\hline $\mathrm{Hp} 3$ & 1331328 & $G$ & A & SNP & synonymous & HPATCC43504_01275 & & \\
\hline Hр3 & 1362543 & ACCCCCCCCCCCCCC & ACGCCCCCCCCCCCCCCCCCCC & indel & nonframeshift insertion & HPB8_1119 & & \\
\hline $\mathrm{Hp} 3$ & 1482699 & GAAAAAA & GAAAAAAAA & indel & intergenic & & HPG27_298 & \\
\hline Hp3 & 1483269 & A & G & SNP & nonsynonymous & HPG27_298 & & \\
\hline Hp3 & 1592787 & TGGGGGGGGGGGGG & & indel & nonframeshift substitution & HP1354 & & \\
\hline Hp3 & 1593602 & TGGGGGGGGGGG & TGGGGGGGGGGGG & indel & frameshift insertion & HP1354 & & \\
\hline $\mathrm{Hp} 3$ & 1608140 & TGA & TGGA & indel & frameshift substitution & HP1369m & & \\
\hline Нр3 & 1627016 & TGGGGGGGGGGGGG & TGGGGGGGGGGGGGGGG & indel & intergenic & & HP1406 & HELPY_1371 \\
\hline Hр3 & 1640915 & CGAGAGAGAGAGAGA & CGAGAGAGAGAGAGAGA & indel & frameshift insertion & jhp1312 & & \\
\hline Hp3 & 1669898 & $\mathrm{c}$ & $T$ & SNP & synonymous & HP1450 & & \\
\hline Hp4 & 658 & $\mathrm{c}$ & $T$ & SNP & synonymous & HP1529 & & \\
\hline Hp4 & 214663 & CGAGAGAGAGAGAGA & CGAGAGAGAGAGAGAGA & indel & frameshift insertion & HPG27_8 & & \\
\hline Hp4 & 232964 & c & A & SNP & intergenic & & HPPC_00115 & \\
\hline $\mathrm{Hp} 4$ & 249555 & $G$ & A & SNP & nonsynonymous & HP0044 & & \\
\hline Hp4 & 303827 & CGGGGGGGGGGGG & CGGGGGGGGGGGGGG & indel & frameshift insertion & HPP12_0096 & & \\
\hline $\mathrm{Hp} 4$ & 315327 & ACCCCCCCCCC & ACCCCCCCCCCC & indel & intergenic & & HP0103 & \\
\hline Hp4 & 413866 & A & G & SNP & synonymous & HP1535 & & \\
\hline $\mathrm{Hp} 4$ & 413887 & A & G & SNP & synonymous & HP1535 & & \\
\hline Hp4 & 413983 & c & $T$ & SNP & synonymous & HP1535 & & \\
\hline Hp4 & 431032 & c & $T$ & SNP & synonymous & HP0194 & & \\
\hline Hр4 & 437765 & $G$ & $\mathrm{c}$ & SNP & intergenic & & HP0204 & HELPY_0206 \\
\hline Hp4 & 450628 & $G$ & A & SNP & synonymous & HP0213 & & \\
\hline $\mathrm{Hp} 4$ & 455599 & TGGGGGGGGGG & TGGGGGGGGGGG & indel & frameshift insertion & HP0217 & & \\
\hline Hp4 & 483804 & TTAAATACA & $T$ & indel & intergenic & & HPATCC43504_00472 & \\
\hline Hp4 & 546511 & CAAAAAAAAAAAA & CAAAAAAAAAAAAA & indel & intergenic & & HP1243/HPG27_298 & \\
\hline $\mathrm{Hp} 4$ & 548979 & TA & TTGA & indel & intergenic & & jhp1163 & HPG27_298 \\
\hline Hp4 & 581837 & $\begin{array}{l}\text { ATCAAATACTCAAATACTCAAATACTCAAATACTCA } \\
\text { AATAC }\end{array}$ & A & indel & intergenic & & HPATCC43504_00587 & \\
\hline Hp4 & 620169 & $\mathrm{c}$ & $T$ & SNP & nonsynonymous & HP1180 & & \\
\hline Hp4 & 626404 & c & $T$ & SNP & synonymous & HP1175 & & \\
\hline $\mathrm{Hp} 4$ & 627243 & $G$ & A & SNP & nonsynonymous & HP1174 & & \\
\hline Hp4 & 701041 & G & A & SNP & nonsynonymous & HP0499 & & \\
\hline Hp4 & 801836 & GAAAAAAAAAAAA & GAAAAAAAAAAAAAA & indel & intergenic & & jhp0540 & \\
\hline Hp4 & 958702 & CAAAAAAAAAAAAAAAA & CAAAAAAAAAAAAAAAAAAA & indel & intergenic & & HPG27_680 & \\
\hline Hp4 & 1043704 & GTTTTTTTTTTTTTT & GтTTTTTTTTTTTTTTTTT & indel & frameshift insertion/intergenic & HPnc4170 & HP0811 & HPnc4160 \\
\hline Hр4 & 1102157 & GTTTTTTTTTTTT & GTTTTTTTTTTT & indel & intergenic & & HP0876 & HP0875 \\
\hline Hp4 & 1122223 & c & $T$ & SNP & intergenic & & HP0895 & HPnc4510 \\
\hline $\mathrm{Hp} 4$ & 1122228 & TAAAAA & TAAAAAA & indel & intergenic & & HP0895 & HPnc4510 \\
\hline Hp4 & 1124501 & GTTTTTTTTTTTT & GTTTTTTTTTTT & indel & intergenic & & HPG27_1187 & \\
\hline Hp4 & 1187376 & АТTTTTTT & ATTTTTTTT & indel & intergenic & & HP0947 & \\
\hline Hp4 & 1331327 & G & $T$ & SNP & nonsynonymous & HPATCC43504_01275 & & \\
\hline Hр4 & 1331328 & G & A & SNP & synonymous & HPATCC43504_01275 & & \\
\hline Hp4 & 1362543 & ACCCCCCCCCCCCCC & AACCCCCCCCCCCCCCC & indel & frameshift substitution & HPB8_1119 & & \\
\hline $\mathrm{Hp} 4$ & 1483269 & A & $G$ & SNP & nonsynonymous & HPG27_298 & & \\
\hline Hp4 & 1593602 & TGGGGGGGGGGG & TGGGGGGGGGGGG & indel & frameshift insertion & HP1354 & & \\
\hline Hp4 & 1608140 & TGA & TGGA & indel & frameshift substitution & HP1369m & & \\
\hline Hp4 & 1627016 & TGGGGGGGGGGGGG & TGGGGGGGGGGGGGGG & indel & intergenic & & HP1406 & HELPY_1371 \\
\hline Hp4 & 1640915 & CGAGAGAGAGAGAGA & CGAGAGAGAGAGAGAGA & indel & frameshift insertion & jhp1312 & & \\
\hline Hр4 & 1669898 & c & $T$ & SNP & synonymous & HP1450 & & \\
\hline Hp5 & 658 & $\mathrm{c}$ & $T$ & SNP & synonymous & HP1529 & & \\
\hline Hp5 & 60246 & G & A & SNP & synonymous & jhp1371 & & \\
\hline Hp5 & 119015 & A & $\mathrm{c}$ & SNP & nonsynonymous & HP1534 & & \\
\hline Hp5 & 214663 & CGAGAGAGAGAGAGA & CGAGAGAGAGAGAGAGA & indel & frameshift insertion & HPG27_8 & & \\
\hline Hp5 & 232964 & CAAAAAAAAAAAAAAAAAAAAA & CAAAAAAAAAAAAAAAAAAAAAAA & indel & intergenic & & HPPC_00115 & \\
\hline Hр5 & 303827 & CGGGGGGGGGGGG & CGGGGGGGGGGGGGG & indel & frameshift insertion & HPP12_0096 & & \\
\hline Hp5 & 315327 & ACCCCCCCCCC & ACCCCCCCCCCC & indel & intergenic & & HP0103 & \\
\hline $\mathrm{Hp} 5$ & 431032 & c & $T$ & SNP & synonymous & HP0194 & & \\
\hline Hp5 & 437765 & G & c & SNP & intergenic & & HP0204 & HELPY_0206 \\
\hline Hp5 & 450628 & G & A & SNP & synonymous & HP0213 & & \\
\hline Hp5 & 455599 & TGGGGGGGGGG & TTGGGGGGGGGGG & indel & nonframeshift insertion & HP0217 & & \\
\hline Hp5 & 483804 & TTAAATACA & $T$ & indel & intergenic & & HPATCC43504_00472 & \\
\hline Hp5 & 546511 & CAAAAAAAAAAAA & CAAAAAAAAAAAAA & indel & intergenic & & HP1243/HPG27_298 & \\
\hline Hp5 & 581837 & ATCAAATACTCAAATACTCAAATACTCAAATAC & A & indel & intergenic & & HPATCC43504_00587 & \\
\hline Hp5 & 620169 & c & $T$ & SNP & nonsynonymous & HP1180 & & \\
\hline Hp5 & 626404 & c & $T$ & SNP & synonymous & HP1175 & & \\
\hline Hp5 & 627243 & G & A & SNP & nonsynonymous & HP1174 & & \\
\hline Hр5 & 701041 & G & A & SNP & nonsynonymous & HP0499 & & \\
\hline Hр5 & 801836 & GAAAAAAAAAAAA & GAAAAAAAAAAAAAA & indel & intergenic & & jhp0540 & \\
\hline Hp5 & 958574 & CGAGAGAGAGAGA & CGAGAGAGAGAGAGA & indel & intergenic & & HPG27_680 & \\
\hline
\end{tabular}


bioRxiv preprint doi: https://doi.org/10.1101/2020.02.15.950279; this version posted February 21, 2020. The copyright holder for this

preprint (which was not certified by peer review) is the author/funder. All rights reserved. No reuse allowed without permission.

\begin{tabular}{|c|c|c|c|c|c|c|c|c|}
\hline $\begin{array}{l}\text { Strain } \\
\text { name }\end{array}$ & $\begin{array}{l}\text { Position in } \\
\text { ATCC43504 }\end{array}$ & ATCC43504 & Reisolate & & Type of difference & Annotation & $\begin{array}{l}\text { Gene nearby the } \\
\text { intergenic region (\#1) }\end{array}$ & $\begin{array}{l}\text { Gene nearby the } \\
\text { intergenic region (\#2) }\end{array}$ \\
\hline $\mathrm{Hp5}$ & 958702 & CAAAAAAAAAAAAAAAA & CCAAAAAAAAAAAAAAAAAA & indel & intergenic & & HPG27_680 & \\
\hline Hр5 & 984279 & $\mathrm{c}$ & $T$ & SNP & nonsynonymous & HP0751 & & \\
\hline Hp5 & 1043704 & GTTTTTTTTTTTTTT & GTTTTTTTTTTTTTTTTT & indel & frameshift insertion/intergenic & HPnc4170 & HP0811 & HPnc4160 \\
\hline Hp5 & 1102157 & GTTTTTTTTTTTT & GTTTTTTTTTTT & indel & intergenic & & HP0876 & HP0875 \\
\hline $\mathrm{Hp5}$ & 1124501 & GTTTTTTTTTTTT & GTTTTTTTTTTT & indel & intergenic & & HPG27_1187 & \\
\hline $\mathrm{Hp} 5$ & 1187376 & АТTTTTTT & АТTTTTTTT & indel & intergenic & & HP0947 & \\
\hline $\mathrm{Hp5}$ & 1192576 & $G$ & $T$ & SNP & intergenic & & HP0953 & \\
\hline Hp5 & 1284699 & AGGGGGGGGGG & AGGGGGGGGGGG & indel & stopgain & HPG27_1018 & & \\
\hline $\mathrm{Hp5}$ & 1331327 & G & $T$ & SNP & nonsynonymous & HPATCC43504_01275 & & \\
\hline $\mathrm{Hp5}$ & 1331328 & G & A & SNP & synonymous & HPATCC43504_01275 & & \\
\hline Hp5 & 1362543 & ACCCCCCCCCCCCCCC & 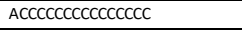 & indel & frameshift insertion & HPB8_1119 & & \\
\hline $\mathrm{Hp} 5$ & 1593602 & TGGGGGGGGGGG & TGGGGGGGGGGGG & indel & frameshift insertion & HP1354 & & \\
\hline Hp5 & 1627016 & TGGGGGGGGGGGGG & TGGGGGGGGGGGGGGGGG & indel & intergenic & & HP1406 & HELPY_1371 \\
\hline $\mathrm{Hp5}$ & 1669898 & $\mathrm{c}$ & $T$ & SNP & synonymous & HP1450 & & \\
\hline Hp6 & 658 & c & $T$ & SNP & synonymous & HP1529 & & \\
\hline Hp6 & 146525 & CAAAAAAAAAAAA & CAAAAAAAAAAAAAAA & indel & intergenic & & HP1397 & \\
\hline Hp6 & 214663 & CGAGAGAGAGAGAGA & CGAGAGAGAGAGAGAGA & indel & frameshift insertion & HPG27_8 & & \\
\hline $\mathrm{Hp} 6$ & 232959 & CTTTA & СТTTCAA & indel & intergenic & & HPPC_00115 & \\
\hline Hр6 & 249268 & $\mathrm{c}$ & $T$ & SNP & nonsynonymous & HPB8_1582 & & \\
\hline $\mathrm{Hp} 6$ & 303827 & CGGGGGGGGGGGG & CGGGGGGGGGGGGGG & indel & frameshift insertion & HPP12_0096 & & \\
\hline Hp6 & 315327 & ACCCCCCCCCC & ACCCCCCCCCCCC & indel & intergenic & & HP0103 & \\
\hline Hр6 & 431032 & c & $\mathrm{T}$ & SNP & synonymous & HP0194 & & \\
\hline Hр6 & 437765 & $\mathrm{G}$ & c & SNP & intergenic & & HP0204 & HELPY_0206 \\
\hline Hp6 & 450628 & $\mathrm{G}$ & $\mathrm{A}$ & SNP & synonymous & HP0213 & & \\
\hline Hр6 & 455599 & TGGGGGGGGGG & TGGGGGGGGGGG & indel & frameshift insertion & HP0217 & & \\
\hline $\mathrm{Hp} 6$ & 483804 & $T$ & TTAAATACA & indel & intergenic & & HPATCC43504_00472 & \\
\hline Hp6 & 546511 & CAAAAAAAAAAAA & CAAAAAAAAAAAAA & indel & intergenic & & HP1243/HPG27_298 & \\
\hline Hр6 & 581837 & ATCAAATACTCAAATACTCAAATACTCAAATAC & A & indel & intergenic & & HPATCC43504_00587 & \\
\hline Hр6 & 620169 & c & $\mathrm{T}$ & SNP & nonsynonymous & HP1180 & & \\
\hline Hp6 & 626404 & c & $\mathrm{T}$ & SNP & synonymous & HP1175 & & \\
\hline Hp6 & 627243 & G & A & SNP & nonsynonymous & HP1174 & & \\
\hline $\mathrm{Hp} 6$ & 701041 & $G$ & A & SNP & nonsynonymous & HP0499 & & \\
\hline Hp6 & 801836 & GAAAAAAAAAAAA & GAAAAAAAAAAAAAA & indel & intergenic & & jhp0540 & \\
\hline Hp6 & 805851 & AT & AATT & indel & intergenic & & HPG27_556 & \\
\hline Hр6 & 958574 & CGAGAGAGAGAGA & CGAGAGAGAGAGAGA & indel & intergenic & & HPG27_680 & \\
\hline Hp6 & 958702 & CAAAAAAAAAAAAAAAA & CAAAAAAAAAAAAAAAAAAA & indel & intergenic & & HPG27_680 & \\
\hline $\mathrm{Hp} 6$ & 984287 & GA & G & indel & frameshift deletion & HP0751 & & \\
\hline Hp6 & 1043704 & GTTTTTTTTTTTTTT & GTTTTTTTTTTTTTTTT & indel & frameshift insertion/intergenic & HPnc4170 & HP0811 & HPnc4160 \\
\hline Hр6 & 1102157 & GTTTTTTTTTTTT & GTTTTTTTTTT & indel & intergenic & & HP0876 & HP0875 \\
\hline Hр6 & 1124346 & CGAGAGAGAGAGAGAGAGA & CGAGAGAGAGAGAGAGA & indel & intergenic & & HPG27_1187 & \\
\hline Hp6 & 1124501 & GTTTTTTTTTTTT & GTTTTTTTTTTT & indel & intergenic & & HPG27_1187 & \\
\hline Hp6 & 1187376 & АТTTTTTT & ATTTTTTTT & indel & intergenic & & HP0947 & \\
\hline Hp6 & 1362543 & ACCCCCCCCCCCCCC & ACGCGCCCCCCCCCCCCCC & indel & frameshift substitution & HPB8_1119 & & \\
\hline Hp6 & 1593602 & TGGGGGGGGGGG & TGGGGGGGGGGGG & indel & frameshift insertion & HP1354 & & \\
\hline Hp6 & 1608125 & TGGGGGGGGGGGGGG & TTGGGGGGGGGGGGGG & indel & nonframeshift substitution & HP1369m & & \\
\hline Hp6 & 1627016 & TGGGGGGGGGGGGG & TGGGGGGGGGGGGGGGGG & indel & intergenic & & HP1406 & HELPY_1371 \\
\hline Hp6 & 1669898 & c & $\mathrm{T}$ & SNP & synonymous & HP1450 & & \\
\hline $\mathrm{Hp} 7$ & 8299 & AGGGGGGGGGGG & AGGGGGGGGGGGG & indel & frameshift insertion & HPAG1_1393 & & \\
\hline $\mathrm{Hp} 7$ & 99327 & $\mathrm{c}$ & $T$ & SNP & synonymous & jhp0935 & & \\
\hline Hp7 & 168379 & $\mathrm{c}$ & $\mathrm{T}$ & SNP & synonymous & HP1547 & & \\
\hline Hp7 & 199597 & c & $\mathrm{T}$ & SNP & intergenic & & HP1582 & jhp1488 \\
\hline $\mathrm{Hp} 7$ & 232964 & c & A & SNP & intergenic & & HPPC_00115 & \\
\hline $\mathrm{Hp} 7$ & 251374 & $\mathrm{G}$ & A & SNP & nonsynonymous & HP0045 & & \\
\hline $\mathrm{Hp} 7$ & 276989 & $G$ & A & SNP & nonsynonymous & HPP12_0070 & & \\
\hline Hp7 & 303827 & CGGGGGGGGGGGG & CGGGGGGGGGGGGGGG & indel & nonframeshift insertion & HPP12_0096 & & \\
\hline Hp7 & 315327 & AcccccccccC & AccccccccccC & indel & intergenic & & HP0103 & \\
\hline $\mathrm{Hp} 7$ & 332924 & TTGTCTTTTGTTTTTCTTGTTC & $\mathrm{T}$ & indel & nonframeshift deletion & HP0119 & & \\
\hline Hp7 & 424517 & CGGGG & CGGGGG & indel & intergenic & & HP0189 & HP0188 \\
\hline $\mathrm{Hp} 7$ & 430922 & G & A & SNP & nonsynonymous & HP0194 & & \\
\hline $\mathrm{Hp} 7$ & 455599 & TGGGGGGGGGG & TGGGGGGGGGGG & indel & frameshift insertion & HP0217 & & \\
\hline $\mathrm{Hp} 7$ & 483804 & TTAAATACA & $\mathrm{T}$ & indel & intergenic & & HPATCC43504_00472 & \\
\hline Hp7 & 546511 & CAAAAAAAAAAAA & CAAAAAAAAAAAAA & indel & intergenic & & HP1243/HPG27_298 & \\
\hline Hp7 & 581837 & ATCAAATACTCAAATACTCAAATACTCAAATAC & A & indel & intergenic & & HPATCC43504_00587 & \\
\hline $\mathrm{Hp} 7$ & 691071 & G & A & SNP & synonymous & HP0489 & & \\
\hline $\mathrm{Hp} 7$ & 691074 & A & c & SNP & synonymous & HP0489 & & \\
\hline Hp7 & 691077 & $\mathrm{G}$ & A & SNP & synonymous & HP0489 & & \\
\hline Hp7 & 691088 & $\mathrm{c}$ & CG & indel & frameshift insertion & HP0489 & & \\
\hline Hp7 & 691101 & $\mathrm{~T}$ & c & SNP & synonymous & HP0489 & & \\
\hline $\mathrm{Hp} 7$ & 691113 & $\mathrm{G}$ & $T$ & SNP & nonsynonymous & HP0489 & & \\
\hline $\mathrm{Hp} 7$ & 691157 & c & A & SNP & nonsynonymous & HP0489 & & \\
\hline $\mathrm{Hp} 7$ & 691173 & $\mathrm{c}$ & A & SNP & synonymous & HP0489 & & \\
\hline $\mathrm{Hp} 7$ & 691175 & A & $G$ & SNP & nonsynonymous & HP0489 & & \\
\hline Hp7 & 691179 & c & G & SNP & synonymous & HP0489 & & \\
\hline $\mathrm{Hp} 7$ & 691185 & A & $G$ & SNP & synonymous & HP0489 & & \\
\hline $\mathrm{Hp} 7$ & 691189 & $\mathrm{c}$ & $G$ & SNP & nonsynonymous & HP0489 & & \\
\hline $\mathrm{Hp} 7$ & 691190 & A & c & SNP & nonsynonymous & HP0489 & & \\
\hline Hp7 & 801836 & GAAAAAAAAAAAA & GAAAAAAAAAAAAA & indel & intergenic & & jhp0540 & \\
\hline $\mathrm{Hp} 7$ & 830053 & c & $\mathrm{T}$ & SNP & nonsynonymous & HPP12_0617 & & \\
\hline Hp7 & 958702 & CAAAAAAAAAAAAAAAA & CAAAAAAAAAAAAAAAAAAA & indel & intergenic & & HPG27_680 & \\
\hline $\mathrm{Hp} 7$ & 1043704 & GTTTTTTTTTTTTTT & GTTTTTTTTTTTTTTTT & indel & frameshift insertion/intergenic & HPnc4170 & HP0811 & HPnc4160 \\
\hline $\mathrm{Hp} 7$ & 1187376 & АТTTTTTT & ATTTTTTTT & indel & intergenic & & HP0947 & \\
\hline $\mathrm{Hp} 7$ & 1262251 & $\mathrm{c}$ & A & SNP & nonsynonymous & jhp0981 & & \\
\hline Hp7 & 1295885 & GATATATATATATATATAT & GATATATATATATATATGTATAT & indel & frameshift insertion & gene_1054 & & \\
\hline Hp7 & 1295885 & GATATATATATATATATAT & GGTATATATATATATATATATAT & indel & frameshift insertion & gene_1054 & & \\
\hline Hр7 & 1332987 & $\mathrm{~T}$ & C & SNP & synonymous & HPATCC 435 & & \\
\hline
\end{tabular}


bioRxiv preprint doi: https://doi.org/10.1101/2020.02.15.950279; this version posted February 21, 2020. The copyright holder for this

preprint (which was not certified by peer review) is the author/funder. All rights reserved. No reuse allowed without permission.

\begin{tabular}{|c|c|c|c|c|c|c|c|c|}
\hline $\begin{array}{l}\text { Strain } \\
\text { name }\end{array}$ & $\begin{array}{l}\text { Position in } \\
\text { ATCC43504 }\end{array}$ & ATCC43504 & uence in & & Type of difference & Annotation & $\begin{array}{c}\text { Gene nearby the } \\
\text { intergenic region (\#1) }\end{array}$ & $\begin{array}{c}\text { Gene nearby the } \\
\text { intergenic region (\#2) }\end{array}$ \\
\hline $\mathrm{Hp} 7$ & 1332996 & $T$ & G & SNP & synonymous & HPATCC43504_01275 & & \\
\hline $\mathrm{Hp} 7$ & 1332997 & G & c & SNP & nonsynonymous & HPATCC43504_01275 & & \\
\hline Hp7 & 1332999 & $\mathrm{~T}$ & c & SNP & synonymous SNV & HPATCC43504_01275 & & \\
\hline Hp7 & 1362543 & 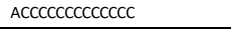 & 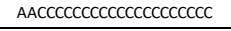 & indel & frameshift substitution & HPB8_1119 & & \\
\hline Hp7 & 1593602 & TGGGGGGGGGGG & TGGGGGGGGGGGG & indel & frameshift insertion & HP1354 & & \\
\hline $\mathrm{Hp} 7$ & 1608125 & TGGGGGGGGGGGGGG & TGGGGGGGGGGGGGGGG & indel & frameshift insertion & HP1369m & & \\
\hline $\mathrm{Hp} 7$ & 1627016 & TGGGGGGGGGGGGG & TGGGGGGGGGGGGGGGG & indel & intergenic & & HP1406 & HELPY_1371 \\
\hline Hp8 & 658 & $\mathrm{c}$ & $\mathrm{T}$ & SNP & synonymous & HP1529 & & \\
\hline Hp8 & 214663 & CGAGAGAGAGAGAGA & CGAGAGAGAGAGAGAGA & indel & frameshift insertion & HPG27_8 & & \\
\hline Hр8 & 249555 & G & A & SNP & nonsynonymous & HP0044 & & \\
\hline Hp8 & 303827 & CGGGGGGGGGGGG & CGGGGGGGGGGGGGG & indel & frameshift insertion & HPP12_0096 & & \\
\hline Hp8 & 315327 & ACCCCCCCCCC & ACCCCCCCCCCCC & indel & intergenic & & HP0103 & \\
\hline Hp8 & 413866 & A & $G$ & SNP & synonymous & HP1535 & & \\
\hline Hp8 & 413887 & A & G & SNP & synonymous & HP1535 & & \\
\hline Hp8 & 413983 & $\mathrm{c}$ & $\mathrm{T}$ & SNP & synonymous & HP1535 & & \\
\hline Hp8 & 431032 & c & $\mathrm{T}$ & SNP & synonymous & HP0194 & & \\
\hline Hp8 & 437765 & G & c & SNP & intergenic & & HP0204 & HELPY_0206 \\
\hline Hр8 & 444106 & GITTTTTTTTTT & GTTTTTTTTTTTTT & indel & intergenic & & HP0209 & HPPC_01040 \\
\hline Hp8 & 450628 & $G$ & A & SNP & synonymous & HP0213 & & \\
\hline Hp8 & 455599 & TGGGGGGGGGG & TGGGGGGGGGGG & indel & frameshift insertion & HP0217 & & \\
\hline Hp8 & 483804 & TTAAATACA & $\mathrm{T}$ & indel & intergenic & & HPATCC43504_00472 & \\
\hline Hp8 & 546511 & CAAAAAAAAAAAA & CAAAAAAAAAAAAA & indel & intergenic & & HP1243/HPG27_298 & \\
\hline Hp8 & 548979 & TA & TTGA & indel & intergenic & & jhp1163 & HPG27_298 \\
\hline Hp8 & 581837 & ATCAAATAC & A & indel & intergenic & & HPATCC43504_00587 & \\
\hline Hp8 & 620169 & $\mathrm{c}$ & $T$ & SNP & nonsynonymous & HP1180 & & \\
\hline Hp8 & 626404 & $\mathrm{c}$ & $\mathrm{T}$ & SNP & synonymous & HP1175 & & \\
\hline Hp8 & 627243 & G & A & SNP & nonsynonymous & HP1174 & & \\
\hline Hp8 & 701041 & G & A & SNP & nonsynonymous & HP0499 & & \\
\hline Hp8 & 801836 & GAAAAAAAAAAAA & GAAAAAAAAAAAAAA & indel & intergenic & & jhp0540 & \\
\hline Hр8 & 958702 & CAAAAAAAAAAAAAAAA & CAAAAAAAAAAAAAAAAAAA & indel & intergenic & & HPG27_680 & \\
\hline Hp8 & 1043704 & GTTTTTTTTTTTTTT & GTTTTTTTTTTTTTTTTTT & indel & frameshift insertion/intergenic & HPnc4170 & HP0811 & HPnc4160 \\
\hline Hp8 & 1102157 & GTTTTTTTTTTTT & GTTTTTTTTTTT & indel & intergenic & & HP0876 & HP0875 \\
\hline Hp8 & 1122223 & $\mathrm{c}$ & $\mathrm{T}$ & SNP & intergenic & & HP0895 & HPnc4510 \\
\hline Hp8 & 1122228 & TAAAAA & TAAAAAA & indel & intergenic & & HP0895 & HPnc4510 \\
\hline Hp8 & 1124501 & GTTTTTTTTTTTT & GTTTTTTTTTTT & indel & intergenic & & HPG27_1187 & \\
\hline Hр8 & 1187376 & АТTTTTTT & ATTTTTTTT & indel & intergenic & & HP0947 & \\
\hline Hp8 & 1331328 & $G$ & A & SNP & synonymous & HPATCC43504_01275 & & \\
\hline Hp8 & 1362543 & ACCCCCCCCCCCCCC & AсCCCCCCCCCCCCCC & indel & frameshift insertion & HPB8_1119 & & \\
\hline Hp8 & 1482848 & G & $\mathrm{T}$ & SNP & nonsynonymous & HPG27_298 & & \\
\hline Hp8 & 1483202 & G & A & SNP & nonsynonymous & HPG27_298 & & \\
\hline Hp8 & 1483269 & A & G & SNP & nonsynonymous & HPG27_298 & & \\
\hline Hр8 & 1484980 & G & A & SNP & synonymous & HPATCC43504_01426 & & \\
\hline Hp8 & 1484991 & ATTTTTTTT & ACTCTTTTTTTT & indel & nonframeshift insertion & HPATCC43504_01426 & & \\
\hline Hp8 & 1582075 & GTTTTTTTTTTTT & GATTTTTTTTTTTTTTT & indel & intergenic & & HELPY_1317 & HP0228 \\
\hline Hp8 & 1593602 & TGGGGGGGGGGG & TGGGGGGGGGGGG & indel & frameshift insertion & HP1354 & & \\
\hline Hp8 & 1608140 & TGA & TGGA & indel & frameshift substitution & HP1369m & & \\
\hline Hp8 & 1627016 & TGGGGGGGGGGGGG & TGGGGGGGGGGGGGGG & indel & intergenic & & HP1406 & HELPY_1371 \\
\hline Hp8 & 1640915 & CGAGAGAGAGAGAGA & CGAGAGAGAGAGAGAGA & indel & frameshift insertion & jhp1312 & & \\
\hline Hp8 & 1669898 & $\mathrm{c}$ & $T$ & SNP & synonymous & HP1450 & & \\
\hline Hp9 & 8299 & AGGGGGGGGGGG & AGGGGGGGGGGGG & indel & frameshift insertion & HPAG1_1393 & & \\
\hline Hp9 & 99327 & $\mathrm{c}$ & $\mathrm{T}$ & SNP & synonymous & jhp0935 & & \\
\hline Hp9 & 168379 & c & $\mathrm{T}$ & SNP & synonymous & HP1547 & & \\
\hline Hp9 & 199597 & c & $\mathrm{T}$ & SNP & intergenic & & HP1582 & jhp1488 \\
\hline Hр9 & 232964 & c & A & SNP & intergenic & & HPPC_00115 & \\
\hline Hp9 & 276989 & $G$ & A & SNP & nonsynonymous & HPP12_0070 & & \\
\hline Hp9 & 303827 & CGGGGGGGGGGGG & CGGGGGGGGGGGGGGGG & indel & frameshift insertion & HPP12_0096 & & \\
\hline Hp9 & 315327 & AсCСCCCCCCC & AсCесCесCеCC & indel & intergenic & & HP0103 & \\
\hline Hp9 & 430922 & G & A & SNP & nonsynonymous & HP0194 & & \\
\hline Hp9 & 444106 & GITTTTTTTTTT & GтTTTTTTTTTTTTT & indel & intergenic & & HP0209 & HPPC_01040 \\
\hline Hp9 & 455599 & TGGGGGGGGGG & TGGGGGGGGGGG & indel & frameshift insertion & HP0217 & & \\
\hline Hp9 & 464722 & $T$ & G & SNP & synonymous & HP0227 & & \\
\hline Hp9 & 464740 & $\mathrm{c}$ & A & SNP & synonymous & HP0227 & & \\
\hline Hp9 & 464743 & A & G & SNP & synonymous & HP0227 & & \\
\hline Hp9 & 483804 & TTAAATACA & $T$ & indel & intergenic & & HPATCC43504_00472 & \\
\hline Hp9 & 546511 & CAAAAAAAAAAAA & CAAAAAAAAAAAAA & indel & intergenic & & HP1243/HPG27_298 & \\
\hline Hр9 & 581837 & ATCAAATACTCAAATAC & A & indel & intergenic & & HPATCC43504_00587 & \\
\hline Hp9 & 691071 & $G$ & A & SNP & synonymous & HP0489 & & \\
\hline Hp9 & 691074 & A & c & SNP & synonymous & HP0489 & & \\
\hline Hp9 & 691077 & G & A & SNP & synonymous & HP0489 & & \\
\hline Hp9 & 691089 & A & G & SNP & synonymous & HP0489 & & \\
\hline Hp9 & 691091 & $\mathrm{c}$ & A & SNP & nonsynonymous & HP0489 & & \\
\hline Hp9 & 691101 & $\mathrm{~T}$ & c & SNP & synonymous & HP0489 & & \\
\hline Hp9 & 691113 & $G$ & $T$ & SNP & nonsynonymous & HP0489 & & \\
\hline Hp9 & 691173 & $\mathrm{c}$ & A & SNP & synonymous & HP0489 & & \\
\hline Нp9 & 691175 & A & G & SNP & nonsynonymous & HP0489 & & \\
\hline Нр9 & 691179 & c & G & SNP & synonymous & HP0489 & & \\
\hline Hp9 & 691185 & A & G & SNP & synonymous & HP0489 & & \\
\hline Hр9 & 691189 & c & G & SNP & nonsynonymous & HP0489 & & \\
\hline Hp9 & 691190 & A & c & SNP & nonsynonymous & HP0489 & & \\
\hline Hр9 & 801836 & GAAAAAAAAAAAA & GAAAAAAAAAAAAA & indel & intergenic & & jhp0540 & \\
\hline Нp9 & 830053 & $\mathrm{c}$ & $\mathrm{T}$ & SNP & nonsynonymous & HPP12_0617 & & \\
\hline Hр9 & 879985 & c & $T$ & SNP & nonsynonymous & HP0656 & & \\
\hline Hp9 & 958702 & CAAAAAAAAAAAAAAAA & CAAAAAAAAAAAAAAAAAA & indel & intergenic & & HPG27_680 & \\
\hline Hp9 & 1043704 & GTTTTTTTTTTTTTT & GтTTTTTTTTTTTTTTTT & indel & frameshift insertion/intergenic & HPnc4170 & HP0811 & HPnc4160 \\
\hline Hp9 & 1139646 & GTTTTTTTTTTTT & GTTTTTTTTTTTTT & indel & intergenic & & HPP12_0910 & \\
\hline
\end{tabular}


bioRxiv preprint doi: https://doi.org/10.1101/2020.02.15.950279; this version posted February 21, 2020. The copyright holder for this preprint (which was not certified by peer review) is the author/funder. All rights reserved. No reuse allowed without permission.

\begin{tabular}{|c|c|c|c|c|c|c|c|c|}
\hline $\begin{array}{l}\text { Strain } \\
\text { name }\end{array}$ & $\begin{array}{l}\text { Position in } \\
\text { ATCC43504 }\end{array}$ & ATCC43504 & Reisolate & & Type of difference & Annotation & $\begin{array}{c}\text { Gene nearby the } \\
\text { intergenic region (\#1) }\end{array}$ & $\begin{array}{l}\text { Gene nearby the } \\
\text { intergenic region (\#2) }\end{array}$ \\
\hline Hр9 & 1187376 & АТTTTTTT & ATTTTTTTT & indel & intergenic & & HP0947 & \\
\hline Hp9 & 1192157 & $T$ & C & SNP & nonsynonymous & HP0953 & & \\
\hline Hр9 & 1331328 & $\mathrm{G}$ & $\mathrm{A}$ & SNP & synonymous & HPATCC43504_01275 & & \\
\hline Hp9 & 1332987 & $\mathrm{~T}$ & c & SNP & synonymous & HPATCC43504_01275 & & \\
\hline Hp9 & 1332996 & $T$ & G & SNP & synonymous & HPATCC43504_01275 & & \\
\hline Hр9 & 1332997 & G & c & SNP & nonsynonymous & HPATCC43504_01275 & & \\
\hline $\mathrm{Hp9}$ & 1332999 & $\mathrm{~T}$ & $\mathrm{c}$ & SNP & synonymous SNV & HPATCC43504_01275 & & \\
\hline $\mathrm{Hp9}$ & 1362543 & $\mathrm{ACCC} C \mathrm{CCC} C \mathrm{CCCCC}$ & 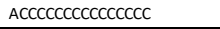 & indel & frameshift insertion & HPB8_1119 & & \\
\hline Hp9 & 1593602 & TGGGGGGGGGGG & TGGGGGGGGGGGG & indel & frameshift insertion & HP1354 & & \\
\hline Hp9 & 1627016 & TGGGGGGGGGGGGG & TGGGGGGGGGGGGGGGG & indel & intergenic & & HP1406 & HELPY_1371 \\
\hline Hp10 & 658 & c & $\mathrm{T}$ & SNP & synonymous & HP1529 & & \\
\hline Hp10 & 8299 & AGGGGGGGGGGG & AGGGGGGGGGGGG & indel & frameshift insertion & HPAG1_1393 & & \\
\hline Hp10 & 214663 & CGAGAGAGAGAGAGA & CGAGAGAGAGAGAGAGA & indel & frameshift insertion & HPG27_8 & & \\
\hline Hp10 & 232959 & СTTTA & CTTTCA & indel & intergenic & & HPPC_00115 & \\
\hline Hp10 & 303827 & CGGGGGGGGGGGG & CGGGGGGGGGGGG & indel & frameshift insertion & HPP12_0096 & & \\
\hline Hp10 & 315327 & ACCCCCCCCCC & ACCCCCCCCCCC & indel & intergenic & & HP0103 & \\
\hline Hp10 & 431032 & c & $T$ & SNP & synonymous & HP0194 & & \\
\hline Hp10 & 437765 & G & c & SNP & intergenic & & HP0204 & HELPY_0206 \\
\hline Hp10 & 450628 & $G$ & A & SNP & synonymous & HP0213 & & \\
\hline Hp10 & 455599 & TGGGGGGGGGG & TGGGGGGGGGGG & indel & frameshift insertion & HP0217 & & \\
\hline Hp10 & 483804 & TTAAATACATAAATACATAAATACA & $\mathrm{T}$ & indel & intergenic & & HPATCC43504_00472 & \\
\hline Hp10 & 546114 & TATTTA & $\mathrm{T}$ & indel & intergenic & & HP1243/HPG27_298 & \\
\hline Hp10 & 546140 & A & AATCTTATTTG & indel & intergenic & & HP1243/HPG27_298 & \\
\hline Hp10 & 546511 & CAAAAAAAAAAAA & CAAAAAAAAAAAAAA & indel & intergenic & & HP1243/HPG27_298 & \\
\hline Hp10 & 581837 & ATCAAATACTCAAATACTCAAATAC & A & indel & intergenic & & HPATCC43504_00587 & \\
\hline Hp10 & 620169 & $\mathrm{c}$ & $\mathrm{T}$ & SNP & nonsynonymous & HP1180 & & \\
\hline Hp10 & 626404 & $\mathrm{c}$ & $\mathrm{T}$ & SNP & synonymous & HP1175 & & \\
\hline Hp10 & 627243 & G & A & SNP & nonsynonymous & HP1174 & & \\
\hline Hp10 & 701041 & G & A & SNP & nonsynonymous & HP0499 & & \\
\hline Hp10 & 801836 & GAAAAAAAAAAAA & GAAAAAAAAAAAAA & indel & intergenic & & jhp0540 & \\
\hline Hp10 & 958702 & CAAAAAAAAAAAAAAAA & & indel & intergenic & & HPG27_680 & \\
\hline Hp10 & 1043704 & GTTTTTTTTTTTTTT & GTTTTTTTTTTTTTTTT & indel & frameshift insertion/intergenic & HPnc4170 & HP0811 & HPnc4160 \\
\hline Hp10 & 1102157 & GTTTTTTTTTTTT & GTTTTTTTTTTT & indel & intergenic & & HP0876 & HP0875 \\
\hline Hp10 & 1124501 & GTTTTTTTTTTTT & GTTTTTTTTTTT & indel & intergenic & & HPG27_1187 & \\
\hline Hp10 & 1187376 & АТTTTTTT & ATTTTTTTT & indel & intergenic & & HP0947 & \\
\hline Hр10 & 1302389 & $\mathrm{G}$ & A & SNP & synonymous & HP0364 & & \\
\hline Hp10 & 1319683 & G & c & SNP & nonsynonymous & jhp1032 & & \\
\hline Hp10 & 1319839 & CAAAAAAAAAAAAA & CAAAAAAAAAAAA & indel & intergenic & & HELPY_1075 & jhp1032 \\
\hline Hp10 & 1331328 & G & A & SNP & synonymous & HPATCC43504_01275 & & \\
\hline Hp10 & 1362543 & AсcсссссессессеC & 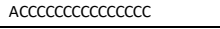 & indel & frameshift insertion & HPB8_1119 & & \\
\hline Hp10 & 1410493 & G & A & SNP & nonsynonymous & jhp0373 & & \\
\hline Hp10 & 1592785 & TGTG & $\mathrm{T}$ & indel & nonframeshift deletion & HP1354 & & \\
\hline Hp10 & 1593602 & TGGGGGGGGGGG & TGGGGGGGGGGGG & indel & frameshift insertion & HP1354 & & \\
\hline Hp10 & 1608125 & TGGGGGGGGGGGGGG & TGGGGGGGGGGGGGGGG & indel & nonframeshift insertion & HP1369m & & \\
\hline Hp10 & 1627016 & TGGGGGGGGGGGG & TGGGGGGGGGGGGGGGG & indel & intergenic & & HP1406 & HELPY_1371 \\
\hline Hр10 & 1669898 & c & $T$ & SNP & synonymous & HP1450 & & \\
\hline
\end{tabular}


bioRxiv preprint doi: https://doi.org/10.1101/2020.02.15.950279; this version posted February 21, 2020. The copyright holder for this

preprint (which was not certified by peer review) is the author/funder. All rights reserved. No reuse allowed without permission.

\begin{tabular}{|c|c|c|c|c|}
\hline \multirow[b]{2}{*}{ Strain name } & \multicolumn{4}{|c|}{ linical isolates obtained from gastric cancer patients (C) } \\
\hline & $\begin{array}{l}\text { Isolation } \\
\text { country }\end{array}$ & $\begin{array}{c}\text { Host } \\
\text { pathology }\end{array}$ & Source & $\begin{array}{l}\text { No. of T- } \\
\text { repeat }\end{array}$ \\
\hline$\overline{\text { CPYG271 }}$ & Japan & Cancer & GCA_000274665.1 & 11 \\
\hline F57 & Japan & Cancer & GCA_-000270065.1 & 11 \\
\hline HE_C13 & Sweden & Cancer & SAMN08381257 & 11 \\
\hline ELS37 & Elsalvador & Cancer & GCA_000255955.1 & 12 \\
\hline VN0464 & Vietnam & Cancer & Registration in progress & 12 \\
\hline xz274 & China & Cancer & GCA_000262655.1 & 12 \\
\hline HE_C52 & Sweden & Cancer & SAMN08381260 & 13 \\
\hline SIM180 & Peru & Cancer & GCA_000148855.1 & 13 \\
\hline vN0232 & Vietram & Cancer & Registration in progress & \\
\hline vN0246 & Vietnam & Cancer & Registration in progress & 13 \\
\hline vN0274 & Vietnam & Cancer & Registration in progress & 13 \\
\hline vN0390 & Vietram & Cancer & Registration in progress & 13 \\
\hline 132 & Singapore & Cancer & SAMN05771043 & 14 \\
\hline $132 \mathrm{~A}$ & Singapore & Cancer & SAMN05804595 & 14 \\
\hline C664 & China & Cancer & SAMN03013094 & 14 \\
\hline CPY6261 & Japan & Cancer & SAMN00777167 & 14 \\
\hline CPY6311 & Japan & Cancer & SAMN00777266 & 14 \\
\hline GC30_HL & France & Cancer & SAMN08381231 & 14 \\
\hline н9 & Singapore & Cancer & SAMN05804593 & 14 \\
\hline Hบо39 & China & Cancer & SAMN02597427 & 14 \\
\hline vN0219 & Vietnam & Cancer & Registration in progress & 14 \\
\hline vNo224 & Vietnam & Cancer & Registration in progress & 14 \\
\hline vNo227 & Vietnam & Cancer & Registration in progress & 14 \\
\hline vN0240 & Vietram & Cancer & Registration in progress & 14 \\
\hline vN0352 & Vietnam & Cancer & Registration in progress & 14 \\
\hline vN0355 & Vietnam & Cancer & Registration in progress & 14 \\
\hline vN0401 & Vietnam & Cancer & Registration in progress & 14 \\
\hline vN0403 & Vietnam & Cancer & Registration in progress & 14 \\
\hline vN0405 & Vietnam & Cancer & Registration in progress & 14 \\
\hline vN0448 & Vietnam & Cancer & Registration in progress & 14 \\
\hline 1177 & Singapore & Cancer & SAMN05791070 & \\
\hline 22402 & Colombia & Cancer & SAMN05405368 & 15 \\
\hline 26093 & Colombia & Cancer & SAMN05394518 & 15 \\
\hline 26100 & Colombia & Cancer & SAMN05393257 & 15 \\
\hline GC69_HL & France & Cancer & SAMN08381240 & 15 \\
\hline HE_C30 & Sweden & Cancer & SAMN08381272 & 15 \\
\hline PeCan18 & Peru & Cancer & SAMN02603196 & 15 \\
\hline PeCan 4 & Peru & Cancer & SAMN02603203 & 15 \\
\hline VNO212 & Vietnam & Cancer & Registration in progress & 15 \\
\hline vNo220 & Vietnam & Cancer & Registration in progress & 15 \\
\hline vNo228 & Vietnam & Cancer & Registration in progress & 15 \\
\hline vNo229 & Vietnam & Cancer & Registration in progress & 15 \\
\hline vNo272 & Vietnam & Cancer & Registration in progress & 15 \\
\hline vN0411 & Vietnam & Cancer & Registration in progress & 15 \\
\hline VN0472 & Vietnam & Cancer & Registration in progress & 15 \\
\hline 11106299 & Japan & Cancer & LC497495 & 15 \\
\hline 178 & Singapore & Cancer & SAMN05771044 & 16 \\
\hline 30950 & Belgium & Cancer & SAMN08381243 & 16 \\
\hline $98-10$ & Japan & Cancer & SAMN02472069 & 16 \\
\hline н30 & Singapore & Cancer & SAMN05806843 & 16 \\
\hline $5468 \mathrm{~A}$ & Singapore & Cancer & SAMN05804677 & 16 \\
\hline VN0235 & Vietram & Cancer & Registration in progress & 16 \\
\hline VN0264 & Vietnam & Cancer & Registration in progress & 16 \\
\hline VN0271 & Vietnam & Cancer & Registration in progress & 16 \\
\hline VN0348 & Vietram & Cancer & Registration in progress & 16 \\
\hline VN0361 & Vietnam & Cancer & Registration in progress & 16 \\
\hline 26084 & Colombia & Cancer & SAMN05395355 & 17 \\
\hline & & & & \\
\hline HE_C58 & Sweden & Cancer & & \\
\hline & & & & \\
\hline & am & & & \\
\hline & & & & \\
\hline & & & & \\
\hline
\end{tabular}

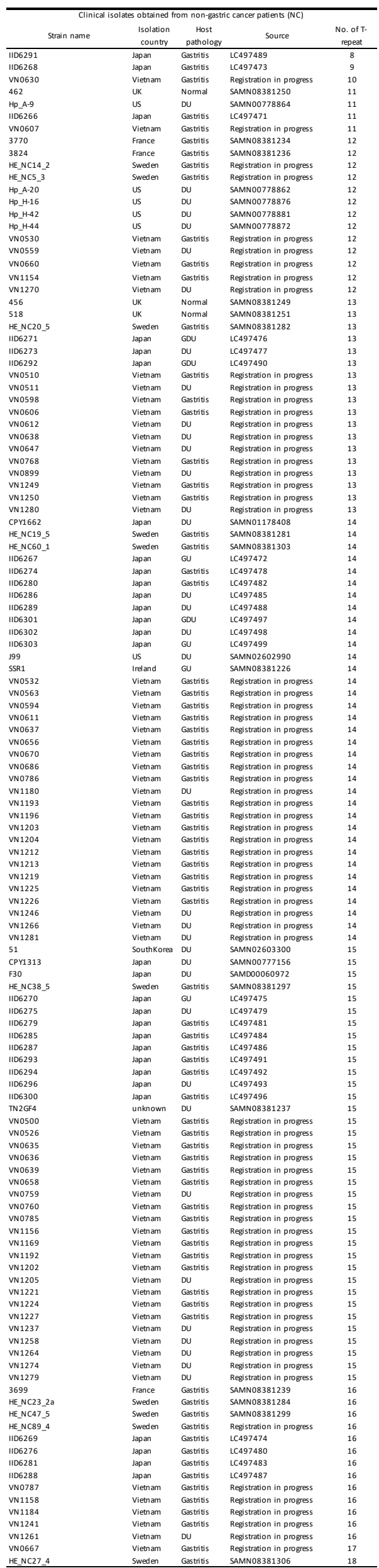


bioRxiv preprint doi: https://doi.org/10.1101/2020.02.15.950279; this version posted February 21, 2020. The copyright holder for this preprint (which was not certified by peer review) is the author/funder. All rights reserved. No reuse allowed without permission.

Supplementary Information 6 | Primers used in this study.

\begin{tabular}{|c|c|c|c|c|c|c|}
\hline \multirow[t]{2}{*}{ Purpose } & \multirow[t]{2}{*}{ Description } & \multirow[t]{2}{*}{ Primer (Forward) } & \multicolumn{3}{|c|}{ Primer (Reverse) } & \multirow[t]{2}{*}{ Reference } \\
\hline & & & Sequence $\left(5^{\prime}-3^{\prime}\right)$ & & Sequence $\left(5^{\prime}-3^{\prime}\right)$ & \\
\hline \multirow{27}{*}{$\begin{array}{l}\text { RT-PCR for } H . \text { pylori mRNA } \\
\text { quantitative analysis } \\
\text { (Figures la, 2d, 4a, 4g; } \\
\text { Extended Data Figures la, } \\
2 \mathrm{f}, 4 \mathrm{~b}, 4 \mathrm{c}, 4 \mathrm{~d}, 7 \mathrm{~b}, 7 \mathrm{c})\end{array}$} & HPnc4160 & HPnc4160 det-s & AAATGTCCTGACGCTCTTACC & UNIVERSAL-as & GAATCGAGCACCAGTTACGC & $\begin{array}{l}\text { This study, miScript II RT } \\
\text { Kit (QIAGEN) }\end{array}$ \\
\hline & HPnc4170 & HPnc4170 det-s & CттсGсCCAGCAтtaAacc & UNIVERSAL-as & GAATCGAGCACCAGTTACGC & $\begin{array}{l}\text { This study, miScript II RT } \\
\text { Kit (QIAGEN) }\end{array}$ \\
\hline & jhp0540 & jhp0540 det-s & TGCTTGTTTGTCCTAAGGCTTC & jhp0540 det-as & TCIGCCCTTGTTTTGCAACG & This study \\
\hline & jhpp163 & jhp1 163 det-s & AACCGCTGAGCAACAAAACG & jhp1163 det-as & ACTCTATGATCATGCTGTGGATTTC & This study \\
\hline & tpi & HELPY_0197 det-s & CAACAAGCCATCCACGCTATC & HELPY_0197 det-as & AGACGCCCTTGTTGTATGGG & This study \\
\hline & tipB & HP0103 det-s & TTACGCCAAATCCGGACACC & HP0103 det-as & TGGTCGTTCCACTCACATGC & This study \\
\hline & arss & HP0164 (jhp0151) det-s & TGAAATAGGGGATCTGCCTAACG & HP0164 (jhp0151) det-as & TAGGGGTGCGTAGTTCATGC & This study \\
\hline & нР0811 & нР0811 det-s & AGCGATTGTCTCTCTCCTATGG & HP0811 det-as & СACGCTCTGTTTAGTGAAAGGC & This study \\
\hline & нР0947 & нР 0947 det-s & GGCCATGTATGAGCCTGATTTG & нР0947 det-as & сCGCCATCACCATTCTTTCAC & This study \\
\hline & HP1354 & HP1354 det-s & AGGCGCAACAGATGAAAACG & HP1354 det-as & TCAGCCCTTTGGCCATACAC & This study \\
\hline & $\begin{array}{l}\text { pldA } \\
\text { HPB8_818 }\end{array}$ & $\begin{array}{l}\text { HPAG1_0475 (HP0499) det-s } \\
\text { HPB8_818 det-s }\end{array}$ & & $\begin{array}{l}\text { HPAG1_0475 (HP0499) det-as } \\
\text { HPB8_818 det-as }\end{array}$ & CGTTATGATCGCTGGCATGG & This study \\
\hline & hop2 & HPG27_8 det-s & TCAACTCCGCCTCTGATGTG & & GTGCCAGCTAAAGCAATCCC & $\begin{array}{l}\text { This study } \\
\text { The }\end{array}$ \\
\hline & babA & HP1243/HPG27_298 det-s & GGTGGTCCACAGATGGAACC & HP1243/HPG27_298 det-as & GATTGACCAGCTCTTGTGCG & This study \\
\hline & sabA & HPG27_680 det-s & GGCTACCCCACTCAATACGC & HPG27_680 det-as & AтCCTGTGGCTTGAGCTTGC & This study \\
\hline & hpaA & HP0410-hpaA det-s & GCGAGTGGGGGGTTTTATAC & HP0410-hpaA det-as & GAAATGCAACTCCACGGACTC & This study \\
\hline & hopE & HELPY_0660-hopE det-s & TGGCTCTCACGCCAAAATTC & HELPY_0660-hopE det-as & GCTCAGCGGCAAACTATTGG & This study \\
\hline & omp 14 & HP0671 det-s & $\begin{array}{l}\text { GCTGTATTGACGGCGGTAAG } \\
\text { a }\end{array}$ & HР 0671 det-as & GCCGGCATCAAGAATTCGAC & This study \\
\hline & hofc & HP0486 det-s & TACCGAGCAGGAGCAAATGG & HP0486 det-as & ACCATCAGCGATACCACGAC & This study \\
\hline & horB & HPSH_00635-horB det-s & TACCCACGAGTCCCTAGAGC & HPSH_00635-horB det-as & ATCAAAGTGGGGCTTATGGC & This study \\
\hline & $\begin{array}{l}\text { cagA } \\
\text { HP1227 }\end{array}$ & $\begin{array}{l}\text { HPP12_0555-cagA det-s } \\
\text { HP1227 det-s }\end{array}$ & $\begin{array}{l}\text { CCGACTAGGGTTCCGTTCAC } \\
\text { GCGATGAAGACATCAAAGCTTTAG }\end{array}$ & $\begin{array}{l}\text { HPP12_0555-cagA det-as } \\
\text { UNIVERSAL-as }\end{array}$ & $\begin{array}{l}\text { AGCAAGCGTTAGCCGATCTC } \\
\text { GAATCGAGCACCAGTTACGC }\end{array}$ & $\begin{array}{l}\text { This study } \\
\text { This study, miscript II RT } \\
\text { Kit (QIAGEN) }\end{array}$ \\
\hline & HELPY_1262 & HELPY_1262 det-s & GGGATGAGGTAAAGATTGAGCTC & UNIVERSAL-as & GaATCGAGCACCAGTracGC & $\begin{array}{l}\text { This study, miscript II RT } \\
\text { Kit (QIAGEN) }\end{array}$ \\
\hline & 23SrRNA & HP23SrRNA det-s & GACTACTACTAATAGAGCGTTTGGC & UNIVERSAL-as & GAATCGAGCACCAGTTACGC & $\begin{array}{l}\text { This study, miScript II RT } \\
\text { Kit (QIAGEN) }\end{array}$ \\
\hline & iceA 2 & HPATCC43504_00587 det-s & GATGTGGTPACAGCCACTACAC & HPATCC43504_00587 det-as & CTGATGGGCATATTGTTGCTG & This study \\
\hline & tpiA & нР0194 det-s & CTAATTCCAAAGACGCGCTC & HP0194 det-as & GAAGACATTTACCTCACCCATGG & This study \\
\hline & fucr2 2 & HPP12_0096 det-s & GAGCCTAAATTATTAAAGCCAAGCC & HPP12_0096 det-as & GCTTGCGCTGAтATTCTTCCTC & This study \\
\hline & HP1406 & HP1406 det-s & TCGCCATTGTCAAGCCATAAG & HP1406 det-as & GGCGTGAAGTGGTGTTTAAAG & This study \\
\hline & HELPY_L1371 & BELPY_1371 det-s & CATGGAAGCTAAAGGCGAACA & HELPY_1371 det-as & $\begin{array}{l}\text { GCGGTTCCAAATTCGTCAT } \\
\text { (C) }\end{array}$ & This study \\
\hline \multirow{3}{*}{$\begin{array}{l}\text { RT-PCR for mice mRNA } \\
\text { quantitative analysis } \\
\text { (Figure 4f) }\end{array}$} & $\frac{\text { cgtA }}{\text { mouse GAPDH }}$ & $\begin{array}{l}\text { HPP0217 det-s } \\
\text { GAPDH det-s }\end{array}$ & $\begin{array}{l}\text { TGCCTACACAATCGCGATGGTG } \\
\text { GTGTTCTCACCACCATCAG }\end{array}$ & HP0217 det-as & 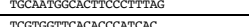 & $\begin{array}{l}\text { This study } \\
\text { Sanada Ty et }\end{array}$ \\
\hline & & & & & & Nature. 2012 \\
\hline & mouse $\mathrm{Cxc12}$ & cxcL2 det-s & CAAGGGTTGACTTCAAGAACATCC & CxcL2 det-as & сCTTGAGAGTGCCTATGACTTC & $\begin{array}{l}\text { Sanada T., et al. } \\
\text { Nature.2012 }\end{array}$ \\
\hline \multirow{4}{*}{$\begin{array}{l}\text { Cloning of } H \text {. pylori } \\
\text { genome fragments for } \\
\text { establishment of deletion } \\
\text { mutants of } H . \text { pylori }\end{array}$} & $\begin{array}{l}\text { cagA deletion mutant } \\
\text { (upstream region) }\end{array}$ & CagA KO up XhoI & $\begin{array}{l}\text { AAAAACCGCTCGAGGAGGGCGAATTGTTAGAA } \\
\text { ACTTG }\end{array}$ & CagA KO up EcoRI & $\begin{array}{l}\text { TTTTTCCGGAATTCGTTTCCAAAAATCTTAAA } \\
\text { GGATTAAGGAATACC }\end{array}$ & This study \\
\hline & $\begin{array}{l}\text { cagA deletion mutant } \\
\text { (downstream region) }\end{array}$ & CagA ko down BamHI & $\begin{array}{l}\text { AAAAACGCGGATCCGGTTTCGTTAGTCATTGT } \\
\text { TTCTCCTPA }\end{array}$ & CagA ko down NotI & $\begin{array}{l}\text { TPTTCCTrTTGCGGCCGCCTACCAATCCATCG } \\
\text { CATGACT }\end{array}$ & This study \\
\hline & $\begin{array}{l}\text { hpnc } 4160 / \text { hpnc } 4170 \text { deletion } \\
\text { mutant (upstream region) }\end{array}$ & HPnc4160/4170 Ko up KpnI & $\begin{array}{l}\text { AAAAAAGGTACCCAAGCCTAATCCCTTACAAA } \\
\text { CCC }\end{array}$ & HPnc4160/4170 KO up ClaI & TPTTTTATCGATCCTTCGCCGAGCATPATACC & This study \\
\hline & $\begin{array}{l}\text { hpnc } 4160 / \text { /pncc4170 deletion } \\
\text { mutant (downstream region) }\end{array}$ & HPnc4160/4170 Ko down BamHI & $\begin{array}{l}\text { AAAAAAGGATCCCTCAACTTTTCACCCAAACT } \\
\text { AACC }\end{array}$ & HPnc4160/4170 Ko down SacI & $\begin{array}{l}\text { TrTPTTGAGCTCGAGCTTTCATTAIIGGCGATG } \\
\text { AATTAG }\end{array}$ & This study \\
\hline $\begin{array}{l}\text { Construction of } \mathrm{T} \text { repeat } \\
\text { mutated } H . \text { pylori }\end{array}$ & $\begin{array}{l}\text { Cloning of T repeat } \\
\text { sequences from H. pylori } \\
\text { isolated from Mongolian } \\
\text { gerbils } 8 \text { wk post } \\
\text { infection into pKSB } \\
\text { plasmid }\end{array}$ & pKSB-HPnc4160 Point mut ApaI & AAAAGGGCCCAGCGATTTGACCTATGATGT & pKSB-HPnc4160 point mut XhoI & AAAACTCGAGACATCTTCTTTCACGCTCTG & This study \\
\hline $\begin{array}{l}\text { Construction of NB-cagA } \\
\text { H. pylori }\end{array}$ & $\begin{array}{l}\text { pKSB-NB-cagA construct } \\
\text { (NB-cagA fragment from } \\
\text { synthesizizd NB-cagA } \\
\text { containing plasmid) }\end{array}$ & pKSB-CagA-NB-ApaI & $\begin{array}{l}\text { AAAAAAGGGCCCGCGACCTTGAAAATTCCGTT } \\
\text { AAAG }\end{array}$ & pKSB-CagA-NB-XhoI & $\begin{array}{l}\text { AAAAAACTCGAGCTGAGCCAATTCTTGATTCC } \\
\text { TTG }\end{array}$ & This study \\
\hline $\begin{array}{l}\text { Estabishment of hpnc } 4160- \\
\text { expressing } H . \text { pylori }\end{array}$ & $\begin{array}{l}\text { pHe12 Plasmid construct } \\
\text { for hppc4160 without } \\
\text { HPnc4170 N terminal region }\end{array}$ & pHe12-4160-de-4170-hed-f XhoI & $\begin{array}{l}\text { GGGGGCTCGAGCAATTAAGGTAAGAGCGTCAG } \\
\mathrm{G}\end{array}$ & pHe12-4160-de-4170-hed-r BanHI & $\begin{array}{l}\text { cccCCGGATCCATCCCATCAATAGGCTTAAAT } \\
\text { TGT }\end{array}$ & This study \\
\hline $\begin{array}{l}\text { Construction of } H . \text { pylori } \\
\text { GST-RNase III-expressing } \\
\text { E. coli }\end{array}$ & pGEX6P-1-Rnase III & PGEX-6P-1 RNaseIII XhoI-f & $\begin{array}{l}\text { AAAATTCGACTCATGAAAAACAAACGCTCTCA } \\
\text { AAARAGC }\end{array}$ & pGEX-6P-1 RNaseIII NotI-r & $\begin{array}{l}\text { AAAACGGCCGCTCATTTGGCTTCCTTCAGTT } \\
\text { TTTGAAGC }\end{array}$ & This study \\
\hline \multirow[t]{10}{*}{$\begin{array}{l}\text { Cloning into pBluescript } \\
\text { plasmid for EMSA }\end{array}$} & $\begin{array}{l}\text { HPnc4160 } \\
\end{array}$ & $\begin{array}{l}\text { Smal1 RNA HPnc4160 Xhor } \\
\end{array}$ & AAAAAACTCGAGGCGAAGGTAAGAGCGAAAG & Smal1 RNA HPnc4160 EcoRI & $\begin{array}{l}\text { AAAAAAGAATTCCAATTAAGGTAAGAGCGTCA } \\
\text { GGA }\end{array}$ & This study \\
\hline & 5 'UTR of hpaA & HP0410 150bp XhoI & $\begin{array}{l}\text { AAAAAACTCGAGGATTGAATGGGAATGATTAT } \\
\text { CTTAAAAAATAGCG }\end{array}$ & HP0410 150bp EcoRI & $\begin{array}{l}\text { AAAAAAGAATTCCCACTCGCTAATAGCGATCC } \\
\text { TA }\end{array}$ & This study \\
\hline & 5 'UTR of hopE & HELPY_0660 15 obp XhoI & $\begin{array}{l}\text { AAAAAACTCGAGTCTPATTCATAAGAGTTAAA } \\
\text { AATATTTCCTTGATT }\end{array}$ & HELPY_0660 150bp EcoRI & $\begin{array}{l}\text { AAAAAAGAATTCGAAGAGCTTAAAACTGCGGA } \\
\text { TAG }\end{array}$ & This study \\
\hline & 5 'UTR of omp14 & HP0671 150bp XhoI & $\begin{array}{l}\text { AAAAAACTCGAGTTPTAAGTTTPAGGGGTGTT } \\
\text { TrTCTTAAGA }\end{array}$ & HP0671 150bp EcoRI & $\begin{array}{l}\text { AAAAAAGAATTCCCTAACACTACCGATAAACA } \\
\text { GAGC }\end{array}$ & This study \\
\hline & 5 'UTR of hofc & HP0486 150bp XhoI & $\begin{array}{l}\text { AAAAAACTCGAGAGAAAAATTAGCTTGATTTT } \\
\text { AAACTAATTCTATATTCT }\end{array}$ & HP0486 150bp EcoRI & $\begin{array}{l}\text { AAAAAAGAATTCGTAAAACGCTTPAGCAATGT } \\
\text { AGC }\end{array}$ & This study \\
\hline & 5 'UTR of horB & HPSH_00635 150bp XhoI & $\begin{array}{l}\text { AAAAAACTCGAGATTCTCATTCAATCGCTATA } \\
\text { TTTAATCAAAAAG }\end{array}$ & HPSH_00635 150bp EcoRI & $\begin{array}{l}\text { AAAAAAGAATTCAAGCTTGTCATTCCTAGAGA } \\
\text { GA }\end{array}$ & This study \\
\hline & 5'UTR of cagA & HPP12_0555 150bp Xhor & $\begin{array}{l}\text { AAAAAACTCGAGAAGACATGAATTCATTACTC } \\
\text { AAGTGTGT }\end{array}$ & HPP12_0555 150bp EcoRI & $\begin{array}{l}\text { AAAAAAGAATTCTPAAAAGCCGCTTCGGTTTG } \\
\mathrm{T}\end{array}$ & This study \\
\hline & 5 'UTR of HP1227 & HP1227 150bp Xhor & $\begin{array}{l}\text { AAAAACCGCTCGAGATGAATGAATAAAATCAT } \\
\text { ATTTATTTCCCTCATTTTCACT }\end{array}$ & HP1227 150bp EcoRI & $\begin{array}{l}\text { TrTTTCCGGAATTCACGCTAAAACGCCTAAAG } \\
\mathrm{c}\end{array}$ & This study \\
\hline & 5'UTR of HELPY_1262 & HELPY_1262 150bp XhoI & $\begin{array}{l}\text { AAAAACCGCTCGAGCTAATGGGGATTTTTTGT } \\
\text { ATTATACCTTAAAACAG }\end{array}$ & HELPY_1262 150bp EcoRI & $\begin{array}{l}\text { AAAAACCGGAATTCCTAACGCCCAAAAGGGTG } \\
\text { AA }\end{array}$ & This study \\
\hline & $\operatorname{CDS}$ of cagA & CagA-B coding Xhor & $\begin{array}{l}\text { AAAAACCGCTCGAGCAATCAAGCAGCAAGTGG } \\
T \text { T }\end{array}$ & CagA-B coding EcoRI & $\begin{array}{l}\text { AAAAACCGGAATTCGCTTCTGATACCGCTTGA } \\
\mathrm{CT}\end{array}$ & is study \\
\hline \multirow[t]{2}{*}{$\begin{array}{l}\text { PCR amplification of DNA } \\
\text { fragments for EMSA }\end{array}$} & NB-cagA fragments & T7 promoter CagA-NB EMSA PCR $s$ & $\begin{array}{l}\text { GACGTTGTAAAACGACGGCCAGTGAGCGCGCG } \\
\text { TAATACACACTCACTATAGGGACCGACTCAATC } \\
\text { AACCACC }\end{array}$ & T7 promoter CagA-NB EMSA PCR as & $\begin{array}{l}\text { GCTTCTGATACCGCTTCACTG } \\
\end{array}$ & This study \\
\hline & all kinds of $D N A$ & T7 promoter EMSA PCR $\mathrm{s}$ & GACGTTGTAAAACGACGGC & T7 promoter EMSA PCR as & CCGGGCTGCAGGAATTC & This study \\
\hline
\end{tabular}

